\title{
Sulfinamide Phosphinates as Chiral Catalysts for the Enantioselective Organocatalytic Reduction of Imines.
}

Ahmed Chelouan, Rocío Recio, Lorenzo G. Borrego, Eleuterio Álvarez, Noureddine Khiar* and Inmaculada Fernández.*

\section{Table of Contents}

$\begin{array}{ll}\text { TOC } & \text { S1 }\end{array}$

Experimental data. $\quad$ S2

${ }^{1} \mathrm{H},{ }^{13} \mathrm{C}$ and ${ }^{31} \mathrm{P}-\mathrm{NMR}$ spectra of selected compounds. $\quad \mathrm{S} 21$

HPLC chromatograms of selected compounds. $\quad$ S63

$\begin{array}{ll}\text { Crystallography Data for selected compounds. } & \mathrm{S} 74\end{array}$ 


\section{Experimental data.}

\section{General Methods.}

All reactions were run under an atmosphere of dry argon using oven-dried glassware and freshly distilled and dried solvents. Toluene, $\mathrm{CH}_{2} \mathrm{Cl}_{2}$, and diethyl ether were dried using molecular sieves, and highest quality solvents were used. Chemicals were obtained from Sigma-Aldrich, and were used without further purification. TLC was carried out on silica gel GF254 (Merck), and compounds were detected by charring with phosphomolybdic acid/EtOH. For flash chromatography, Merck 230-400 mesh silica gel was used. Chromatographic columns were eluted with a positive pressure of air, and eluents are given as volume to volume ratios (v/v). NMR spectra were recorded with BrukerAvance 300 and $500 \mathrm{MHz}$ spectrometers, using $\mathrm{Me}_{4} \mathrm{Si}$ as an internal reference. Chemical shifts are reported in ppm, and coupling constants are reported in Hz. High-resolution mass spectra (HRMS) were recorded in the

Centro de Investigación, Tecnología e Innovación in the University of Seville with a Kratos MS-80RFA 241-MC apparatus. Optical rotations were determined with a Perkin-Elmer 341 polarimeter.

Elemental analyses were measured with a LECO TruSpect CHNS-932 apparatus. Melting points were measured with a Stuart SMP3apparatus in open-ended capillary tubes. Enantiomeric excesses were measured with a Waters alliance 2695 and Agilent Technologies1200 series apparatus with stationary chiral phase columns(Chiralcel®).

X-Ray diffraction data were collected on a Bruker SMART diffractometer APEX graphitemonochromated Mo-Ka radiation, and a Kryo flex low temperatur edevice. Data reduction up $\operatorname{to} \theta=30^{\circ}$ by program the SAINT, corrections for absorption with the program SADABS. The structure was solved by direct methods using SHELXS and refined against $F^{2}$ on all data by full-matrix leasts quares with SHELXL97 using established refinement techniques. 


\section{Synthesis of $N$-Sulfinyl imines. General method.}

To a $0.5 \mathrm{M}$ solution of $\mathrm{Ti}(\mathrm{OEt})_{4}$ in THF (3.8 mmol, 4.4 equiv.) and the corresponding aldehyde (0.87 mmol, 1 equiv.) in THF $(4 \mathrm{~mL})$, at room temperature under argon atmosphere, $(R)$-2-methyl-2-propanesulfinamide $\mathbf{1 R}(1.93 \mathrm{mmol}, 2.2$ equiv.) in THF (1 mL) was added. Once the starting material was consumed (48h), the reaction mixture was hydrolyzed with a saturated $\mathrm{NaCl}$ aqueous solution $(10 \mathrm{~mL})$. The resulting suspension was filtered through a pad of Celite. The aqueous phase was extracted with AcOEt (3 x 40mL) and the combined organic phases were dried with anhydrous $\mathrm{Na}_{2} \mathrm{SO}_{4}$. The solvent was evaporated under reduced pressure and the reaction crude was purified by flash chromatography (AcOEt/Hexano, 1:9) to obtain the desired compound.

\section{$(R)$-(E)-N-[3,5-bis(trifluoromethyl)benzylidene]-2-methyl-2-propanesulfinamide, $3 R$.}

It was prepared following the general procedure from $(R)$ - 2-methyl-2-propanesulfinamide $1 R$ and 3,5-bis(trifluoromethyl)benzaldehyde. The resulting residue was purified by flash chromatography (EtOAc:hexane, 1:3) to give compound $\mathbf{3 R}(288.40 \mathrm{mg}, 96 \%)$ as a white solid: mp: $118^{\circ} \mathrm{C} ;[\alpha]_{\mathrm{D}}{ }^{20}=-95.6\left(c 1.0\right.$ in $\left.\mathrm{CHCl}_{3}\right) ;{ }^{1} \mathrm{H}$ NMR $\left(300 \mathrm{MHz}, \mathrm{CDCl}_{3}\right) \delta 8.67(\mathrm{~s}, 1 \mathrm{H})$, $8.28(\mathrm{~s}, 2 \mathrm{H}), 8.01(\mathrm{~s}, 1 \mathrm{H}), 1.29(\mathrm{~s}, 9 \mathrm{H}) \mathrm{ppm} ;{ }^{13} \mathrm{C} \mathrm{NMR}\left(75 \mathrm{MHz}, \mathrm{CDCl}_{3}\right) \delta 160.2,135.8$, $132.8\left(\mathrm{q}, J_{C P}=6,3 \mathrm{~Hz}\right), 129.1,125.6(\mathrm{~m}), 124.8,121.2,58.6,22.8 \mathrm{ppm}$; IR 1594, 1380, 1267, 1165, 1126, 1083, 998, 904, 759, $700 \mathrm{~cm}^{-1}$; HRMS: calcd for $\mathrm{C}_{13} \mathrm{H}_{14} \mathrm{~F}_{6} \mathrm{NOS}[\mathrm{M}+\mathrm{H}]$ : 346.0700; found: 346.0700 .

\section{$(R)-\mathrm{N}$-[(2'-naphthyl)methylidene]-2-methyl-2-propanesulfinamide, $4 R$.}

It was prepared following the general procedure from $(R)$-2-methyl-2-propanesulfinamide $\mathbf{1} \boldsymbol{R}$ and 2-naphtaldehyde. The resulting residue was purified by flash chromatography (EtOAc:hexane, 1:6) to give compound $\mathbf{4 R}(209.86 \mathrm{mg}, 93 \%)$ as a white solid: $\mathrm{mp} 91-93^{\circ} \mathrm{C}$; $[\alpha]_{\mathrm{D}}{ }^{20}=-146.0\left(c 1.0\right.$ in $\left.\mathrm{CHCl}_{3}\right) ;{ }^{1} \mathrm{H}$ NMR $\left(500 \mathrm{MHz}, \mathrm{CDCl}_{3}\right) \delta 8.75(\mathrm{~s}, 1 \mathrm{H}), 8.20(\mathrm{~s}, 1 \mathrm{H})$, $8.03(\mathrm{dd}, 1 \mathrm{H} . J=1.5$ y $8.5 \mathrm{~Hz}), 7.94(\mathrm{~d}, 1 \mathrm{H}, J=7.9 \mathrm{~Hz}), 7.88(\mathrm{dd}, 2 \mathrm{H}, J=12.2$ y $12.4 \mathrm{~Hz})$, 7.61-7.52 (m, $2 \mathrm{H}), 1.29$ (s, 9H) ppm; ${ }^{13} \mathrm{C}$ NMR (125 MHz, $\left.\mathrm{CDCl}_{3}\right) \delta 162.8,135.5,133.1$, $132.5,131.9,129.2,128.9,128.3,128.1,128,127,123.9,57.8,22.6$ ppm. 


\section{$(R)$-(E)-N-(ferrocenylmethylidene)-2-methyl-2-propanesulfinamide, $5 R$.}

It was prepared following the general procedure from $(R)$-2-methyl-2-propanesulfinamide $\mathbf{1} \boldsymbol{R}$ and ferrocenealdehyde. The resulting residue was purified by flash chromatography (EtOAc:hexane, 1:2) to give compound $\mathbf{5 R}(265 \mathrm{mg}, 96 \%)$ as a yellow solid: $\mathrm{mp}: 100^{\circ} \mathrm{C}$; $[\alpha]_{\mathrm{D}}{ }^{20}=-656.0\left(c 0.16\right.$ in $\left.\mathrm{CH}_{2} \mathrm{Cl}_{2}\right) ;{ }^{1} \mathrm{H}$ NMR $\left(500 \mathrm{MHz}, \mathrm{CDCl}_{3}\right) \delta 8.51(\mathrm{~s}, 1 \mathrm{H}), 4.75-4.74(\mathrm{~m}$, $2 \mathrm{H}), 4.52(\mathrm{~s}, 2 \mathrm{H}), 4.22(\mathrm{~s}, 5 \mathrm{H}), 1.25(\mathrm{~s}, 9 \mathrm{H}) \mathrm{ppm} ;{ }^{13} \mathrm{C} \mathrm{NMR}\left(125 \mathrm{MHz}, \mathrm{CDCl}_{3}\right) \delta 164.4,78.9$, 72.2, 72.2, 70.1, 69.8, 69.4, 57.03, 22.9 ppm; IR 1590, 1455, 1364, 1250, 1106, 1070, 1041, 1029, 1009, 975, 814, 778, $69 \mathrm{~cm}^{-1}$; HRMS: calcd for $\mathrm{C}_{15} \mathrm{H}_{19} \mathrm{FeNONaS}$ : 340.0434; found: 340.0426 .

\section{$(R)$-E-N-(2-methylpropylidene)-2-methyl-2-propanesulfinamide, $6 R$.}

It was prepared following the general procedure from $(R)$-2-methyl-2-propanesulfinamide $\mathbf{1} \boldsymbol{R}$ and isopropaldehyde. The resulting residue was purified by flash chromatography (EtOAc:hexane, $1: 6)$ to give compound $\mathbf{6 R}(123.53 \mathrm{mg}, 81 \%)$ as a yellow liquid: $[\alpha]_{\mathrm{D}}{ }^{20}=-$ $259.4\left(c 1.0\right.$ in $\left.\mathrm{CHCl}_{3}\right)$; ${ }^{1} \mathrm{H}$ NMR $\left(500 \mathrm{MHz}, \mathrm{CDCl}_{3}\right) \delta 7.98(\mathrm{~d}, 1 \mathrm{H}, J=4.3 \mathrm{~Hz}), 2.70(\mathrm{~m}, 1 \mathrm{H})$, $1.18(\mathrm{~s}, 9 \mathrm{H}), 1.15(\mathrm{~d}, 3 \mathrm{H}, \quad J=2.5 \mathrm{~Hz}), 1.13(\mathrm{~d}, 3 \mathrm{H}, J=2.5 \mathrm{~Hz}) \mathrm{ppm} ;{ }^{13} \mathrm{C}$ NMR $(125 \mathrm{MHz}$, $\left.\mathrm{CDCl}_{3}\right) \delta 173.7 .56 .6,35,22.3,19 \mathrm{ppm}$.

\section{Methyl methyl(phenyl)phosphinate, rac-7.}

Dimethyl phenyl phosphonite $(9.4 \mathrm{~g}, 55.0 \mathrm{mmol})$ was added drop-wise to a two neck round bottom flask containing methyl iodide $(0.1 \mathrm{~mL}, 1.6 \mathrm{mmol})$ at a rate to maintain a steady reflux. The reaction was stirred for a further $18 \mathrm{~h}$, then purified via vacuum distillation to afford the title compound as a colourless oil. Yield: 7.7 g, $82 \%$; b.p.: $99-101{ }^{\circ} \mathrm{C} / 0.03$ mmHg (lit.4 $100{ }^{\circ} \mathrm{C} / 0.8 \mathrm{mmHg}$ ); ${ }^{1} \mathrm{H}$ NMR (500 MHz, $\mathrm{CDCl}_{3}$ ): $\delta 7.45-7.41$ (m, 2H), 7.20$7.10(\mathrm{~m}, 3 \mathrm{H}), 3.22$ (d, 3H, $J=11.6 \mathrm{~Hz}), 1.29 \mathrm{ppm}(\mathrm{d}, 3 \mathrm{H}, J=14.3 \mathrm{~Hz}) ;{ }^{13} \mathrm{C} \mathrm{NMR}(125 \mathrm{MHz}$, $\left.\mathrm{CDCl}_{3}\right): \delta 132.1,131.0,13.9,130.2,128.4\left(\mathrm{~d}, J_{\mathrm{PC}}=12.8 \mathrm{~Hz}\right), 50.6\left(\mathrm{~d}, J_{\mathrm{PC}}=5.6 \mathrm{~Hz}\right), 15.1 \mathrm{ppm}$ $\left(\mathrm{d}, J_{\mathrm{PC}}=103.5 \mathrm{~Hz}\right) ;{ }^{31} \mathrm{P} \mathrm{NMR}\left(202 \mathrm{MHz}, \mathrm{CDCl}_{3}\right): \delta+38.3 \mathrm{ppm}$.

\section{Synthesis of Chiral Sulfinamide-Phosphinates. General method (Table 1).}

A $1 \mathrm{M}$ solution of LHMDS in THF (3equiv.) was added to a solution of corresponding racemic methyl phosphinate rac-7 (1 equiv.) in THF under argon atmosphere at $-78^{\circ} \mathrm{C}$. The reaction mixture was stirred at $-78^{\circ} \mathrm{C}$ for $2 \mathrm{~h}$ in order to obtain the corresponding anion. Then, 
this solution was added via cannula to a solution of the corresponding $N$-sulfinylimine (1 equiv.) in $\mathrm{THF}$, previously cooled to $-78^{\circ} \mathrm{C}$. After stirring for $2 \mathrm{~h}$ at $-78^{\circ} \mathrm{C}$, the reaction mixture was hydrolized with a saturated $\mathrm{NH}_{4} \mathrm{Cl}$ aqueous solution $(40 \mathrm{~mL})$. The aqueous phase was then extracted with EtOAc $(5 \times 40 \mathrm{~mL})$ and the combined organic phases were dried with anhydrous $\mathrm{Na}_{2} \mathrm{SO}_{4}$. The solvent was evaporated under reduced pressure and the crude was purified by flash chromatography to give the desired product in each case.

Table 1. Synthesis of organocatalysts 8-12 by addition of phosphinate rac-7 to the $(R)-N$-tertbutylsulfinylaldimines 2-6.

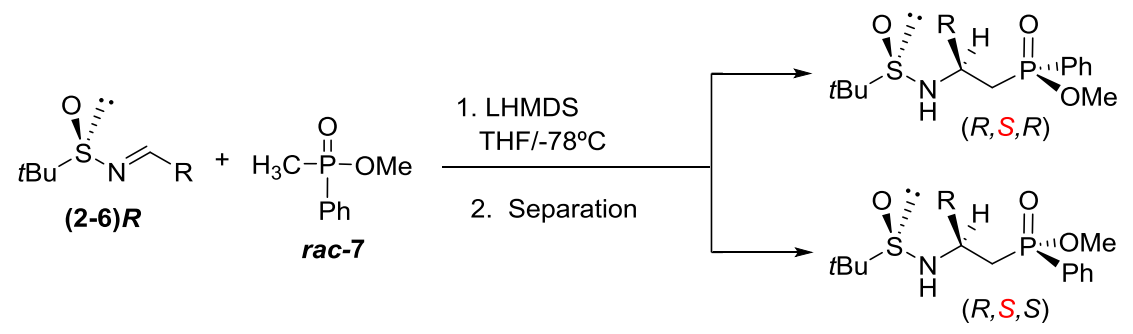

\begin{tabular}{ccccc}
\hline Entry & $\begin{array}{c}\text { Sulfinylimine } \\
(R)\end{array}$ & Product & $\begin{array}{c}(R, S, R) \\
\% \text { Yield }\end{array}$ & $\begin{array}{c}(R, S, S) \\
\% \text { Yield }\end{array}$ \\
\hline 1 & $\mathbf{2}\left(p-\mathrm{MeOC}_{6} \mathrm{H}_{4}\right)$ & $\mathbf{8}$ & $40^{[\mathrm{a}]}$ & $42^{[\mathrm{a}]}$ \\
2 & $\mathbf{3}\left(3,5-\left(\mathrm{CF}_{3}\right)_{2} \mathrm{C}_{6} \mathrm{H}_{3}\right)$ & $\mathbf{9}$ & $50^{[\mathrm{a}]}$ & $50^{[\mathrm{a}]}$ \\
3 & $\mathbf{4}(2-\mathrm{Napht})$ & $\mathbf{1 0}$ & $45^{[\mathrm{a}]}$ & $49^{[\mathrm{a}]}$ \\
4 & $\mathbf{5}(\mathrm{Fc})$ & $\mathbf{1 1}$ & $47^{[\mathrm{b}]}$ & ${ }^{[\mathrm{c}]}$ \\
5 & $\mathbf{6}(i \mathrm{Pr})$ & $\mathbf{1 2}$ & $44^{[\mathrm{b}]}$ & ${ }^{[\mathrm{c}]}$ \\
\hline
\end{tabular}

[a] Isolated by column chromatography. [b] Isolated by fractional crystallization. [c] It could not be isolated in pure diastereomeric form. 

$8(R, S, R)$ and $8(R, S, S)$.

It was prepared following the general procedure, starting from phosphinate rac-7 $(0.7 \mathrm{~g}, 4.13$ mmol), a $1 \mathrm{M}$ solution of LHMDS in THF (12.4 mL, $12.4 \mathrm{mmol})$ and $N$-sulfinylimine $\mathbf{2} \boldsymbol{R}$, (0.99 g, $4.13 \mathrm{mmol})$. The resulting residue was obtained as phosphinates $\mathbf{8}(\boldsymbol{R}, \boldsymbol{S}, \boldsymbol{R})$ and $\mathbf{8}(\boldsymbol{R}, \boldsymbol{S}, \boldsymbol{S})$ in a 1:1 ratio. Purification by column chromatography (EtOAc:hexane, 3:1) gave both diastereomerically pure phosphinates $\mathbf{8}(\boldsymbol{R}, \boldsymbol{S}, \boldsymbol{R})(676.46 \mathrm{mg}, 40 \%)$ and $\mathbf{8}(\boldsymbol{R}, \boldsymbol{S}, \boldsymbol{S})(710.28$ $\mathrm{mg}, 42 \%$ ) as white solids.

Diastereoisomer 8(R,S,R): mp $133^{\circ} \mathrm{C} ;[\alpha]_{\mathrm{D}}{ }^{20}=-3.6\left(c 0.5\right.$ in $\left.\mathrm{CHCl}_{3}\right) ;{ }^{1} \mathrm{H}$ NMR $(500 \mathrm{MHz}$, $\left.\mathrm{CDCl}_{3}\right) \delta 7.65-7.61(\mathrm{~m}, 2 \mathrm{H}) .7 .53-7.50(\mathrm{~m}, 1 \mathrm{H}), 7.42(\mathrm{td}, 2 \mathrm{H}, J=3.4$ y $7.7 \mathrm{~Hz}), 7.25(\mathrm{dt}, 2 \mathrm{H}$, $J=8.7 \mathrm{~Hz}), 6.79(\mathrm{~d}, 2 \mathrm{H}, J=8.7 \mathrm{~Hz}), 4.83(\mathrm{~m}, 1 \mathrm{H}), 4.80(\mathrm{~m}, 1 \mathrm{H}), 3.77(\mathrm{~s}, 3 \mathrm{H}), 3.56(\mathrm{~d}, 3 \mathrm{H}, J=$ $11.1 \mathrm{~Hz}$ ), $2.64(\mathrm{ddd}, 1 \mathrm{H}, J=15.1,10.4$ and $8.2 \mathrm{~Hz}), 2.42(\mathrm{td}, 1 \mathrm{H}, J=15.2$ and $7.3 \mathrm{~Hz}), 1.19$ (s, 9H) ppm; ${ }^{13} \mathrm{C}$ NMR $\left(125 \mathrm{MHz}, \mathrm{CDCl}_{3}\right) \delta 159.3,132 .\left(\mathrm{d}, J_{\mathrm{CP}}=9.6 \mathrm{~Hz}\right), 132.4\left(\mathrm{~d}, J_{\mathrm{CP}}=2.6\right.$ $\mathrm{Hz}), 131.4\left(\mathrm{~d}, J_{\mathrm{CP}}=10.1 \mathrm{~Hz}\right), 130.1\left(\mathrm{~d}, J_{\mathrm{CP}}=125.4 \mathrm{~Hz}\right), 128.7\left(\mathrm{~d}, J_{\mathrm{CP}}=12.6 \mathrm{~Hz}\right), 128.6,113.9$, 56.1, 55.2, $54.4\left(\mathrm{~d}, J_{\mathrm{CP}}=2.6 \mathrm{~Hz}\right), 51\left(\mathrm{~d}, J_{\mathrm{CP}}=6.4 \mathrm{~Hz}\right), 37.5\left(\mathrm{~d}, J_{\mathrm{CP}}=98.5 \mathrm{~Hz}\right), 22.5 \mathrm{ppm} ;{ }^{31} \mathrm{P}$ NMR (202 MHz, $\left.\mathrm{CDCl}_{3}\right) \delta+43.2$ ppm; IR 3212, 2923, 1440, 1614, 1514, 1244, 1180, 885, 826, $751 \mathrm{~cm}^{-1}$;HRMS: calcd for $\mathrm{C}_{20} \mathrm{H}_{29} \mathrm{NO}_{4} \mathrm{PS}[\mathrm{M}+1]$ : 410.1555; found: 410.1539; MS: 393, 392, 290, 289 (100\%), 155, 149, 136, 134, 106, 77.

Diastereoisomer 8(R,S,S): mp $170^{\circ} \mathrm{C} ;[\alpha]_{\mathrm{D}}{ }^{20}=-39.2\left(c 0.5\right.$ in $\left.\mathrm{CHCl}_{3}\right) ;{ }^{1} \mathrm{H}$ NMR $(500 \mathrm{MHz}$, $\left.\mathrm{CDCl}_{3}\right) \delta 7.64-7.60(\mathrm{~m}, 2 \mathrm{H}) .7 .52-7.49(\mathrm{~m}, 1 \mathrm{H}) 7.41(\mathrm{td}, 2 \mathrm{H}, J=3.3$ y $7.7 \mathrm{~Hz}), 7.2(\mathrm{~d}, 2 \mathrm{H}, J=$ $8.6 \mathrm{~Hz}), 6.75(\mathrm{~d}, 2 \mathrm{H}, J=8.2 \mathrm{~Hz}), 4.65(\mathrm{~m}, 1 \mathrm{H}), 4.59$ (d, 1H, J=3.0 Hz), 3.75 (s, 3H), 3.55 (d, $3 \mathrm{H}, J=11.2 \mathrm{~Hz}$ ), $2.82(\mathrm{ddd}, 1 \mathrm{H}, J=8.1,17.2$ y $15.1 \mathrm{~Hz}$ ), 2.33 (ddd, $1 \mathrm{H}, J=5.7,11.3$ y 15.1 $\mathrm{Hz}), 1.20(\mathrm{~s}, 9 \mathrm{H}) \mathrm{ppm} ;{ }^{13} \mathrm{C} \mathrm{NMR}\left(125 \mathrm{MHz}, \mathrm{CDCl}_{3}\right) \delta 159.3,132.9\left(\mathrm{~d}, J_{\mathrm{CP}}=9.9 \mathrm{~Hz}\right), 132.7(\mathrm{~d}$, $\left.J_{\mathrm{CP}}=2.6 \mathrm{~Hz}\right), 131.9\left(\mathrm{~d}, J_{\mathrm{CP}}=10 \mathrm{~Hz}\right), 130.1\left(\mathrm{~d}, J_{\mathrm{CP}}=26.1 \mathrm{~Hz}\right), 129\left(\mathrm{~d}, J_{\mathrm{CP}}=12.6 \mathrm{~Hz}\right), 113.95$, 56, 55.2, 54.2, $51.1\left(\mathrm{~d}, J_{\mathrm{CP}}=6.4 \mathrm{~Hz}\right), 37.4\left(\mathrm{~d}, J_{\mathrm{CP}}=98.2 \mathrm{~Hz}\right), 22.8 \mathrm{ppm} ;{ }^{31} \mathrm{P} \mathrm{NMR}(202 \mathrm{MHz}$, $\left.\mathrm{CDCl}_{3}\right) \delta+43.1 \mathrm{ppm}$; IR 3210, 2950, 1590, 1614, 1512, 1206, 1117, 1032, 1004, 826, $815 \mathrm{~cm}^{-}$ ${ }^{1}$;HRMS: calcd for $\mathrm{C}_{20} \mathrm{H}_{29} \mathrm{NO}_{4} \mathrm{PS}$ [M+1]: 410.1555; found: 410.1539; MS: 393, 392, 290, 289 (100\%), 155, 149, 136, 134, 106, 77. 
phenylphosphinate, $9(R, S, R)$ and $9(R, S, S)$.

It was prepared following the general procedure, starting from phosphinate rac -7 (408.36 mg, 2.40mmol), a 1M solution of LHMDS in THF (7.2 $\mathrm{mL}, 7.2 \mathrm{mmol})$ and $N$-sulfinylimine $\mathbf{3 R}$, (830mg, $2.40 \mathrm{mmol}$ ). The resulting residue was obtained as phosphinates $\mathbf{9}(\boldsymbol{R}, \boldsymbol{S}, \boldsymbol{R})$ and $\mathbf{9}(\boldsymbol{R}, \boldsymbol{S}, \boldsymbol{S})$ in a 1:1 ratio. Purification by column chromatography (EtOAc:ether, 2:1) gave both diastereomerically pure phosphinates $\mathbf{9}(\boldsymbol{R}, \boldsymbol{S}, \boldsymbol{R})(618 \mathrm{mg}, 50 \%)$ and $\mathbf{9}(\boldsymbol{R}, \boldsymbol{S}, \boldsymbol{S})(618.5 \mathrm{mg}, 50 \%)$ as white solids.

Diastereoisomer 9(R,S,R):mp $50^{\circ} \mathrm{C} ;[\alpha]_{\mathrm{D}}{ }^{20}=+2.6\left(c 0.5\right.$ in $\left.\mathrm{CH}_{2} \mathrm{Cl}_{2}\right) ;{ }^{1} \mathrm{H}$ NMR $(500 \mathrm{MHz}$, $\left.\mathrm{CDCl}_{3}\right) \delta 7.77$ (s. $\left.2 \mathrm{H}\right), 7.61$ (s. $\left.1 \mathrm{H}\right), 7.52-7.44(\mathrm{~m}, 3 \mathrm{H}), 7.34$ (td, $1 \mathrm{H}, J=3.5$ y $\left.7.7 \mathrm{~Hz}\right), 5.60$ $(\mathrm{d}, 1 \mathrm{H}, J=6.2 \mathrm{~Hz}), 5.05(\mathrm{dq}, 1 \mathrm{H}, J=6.3$ y $20.5 \mathrm{~Hz}), 3.55$ (d, 3H, $J=11.2 \mathrm{~Hz}), 2.66-2.51(\mathrm{~m}$, 2H), $1.22(\mathrm{~s}, 9 \mathrm{H}) \mathrm{ppm} ;{ }^{13} \mathrm{C} \mathrm{NMR}\left(125 \mathrm{MHz}, \mathrm{CDCl}_{3}\right) \delta 143.8\left(\mathrm{~d}, J_{\mathrm{CP}}=7.5 \mathrm{~Hz}\right), 133\left(\mathrm{~d}, J_{\mathrm{CP}}=\right.$ $2.8 \mathrm{~Hz}), 132.1,131.9,131.7\left(\mathrm{~d}, J_{\mathrm{CP}}=10.2 \mathrm{~Hz}\right), 130.9\left(\mathrm{~d}, J_{\mathrm{CP}}=181.8 \mathrm{~Hz}\right), 129.1\left(\mathrm{~d}, J_{\mathrm{CP}}=12.9\right.$ $\mathrm{Hz}), 127.9,124.5,122.3,122.1,57.1,54.1\left(\mathrm{~d}, J_{\mathrm{CP}}=3.2 \mathrm{~Hz}\right), 51.4\left(\mathrm{~d}, J_{\mathrm{CP}}=6.3 \mathrm{~Hz}\right), 37.2(\mathrm{~d}$, $\left.J_{\mathrm{CP}}=99.4 \mathrm{~Hz}\right), 22.8 \mathrm{ppm} ;{ }^{31} \mathrm{P}$ NMR $\left(202 \mathrm{MHz}, \mathrm{CDCl}_{3}\right) \delta+42.5 \mathrm{ppm}$; IR 3212, 2955, 1623 , 1466, 1438, 1375, 1276, 1171, 1129, 1034, 898, 828, 791, 734, 696, $681 \mathrm{~cm}^{-1}$; HRMS: calcd for $\mathrm{C}_{21} \mathrm{H}_{25} \mathrm{~F}_{6} \mathrm{NO}_{3} \mathrm{PS}$ [M+1]: 516.1197; found: 516.1197.

Diastereoisomer 9(R,S,S): mp $174^{\circ} \mathrm{C} ;[\alpha]_{\mathrm{D}}{ }^{20}=-29.8\left(c 0.5\right.$ in $\left.\mathrm{CH}_{2} \mathrm{Cl}_{2}\right) ;{ }^{1} \mathrm{H}$ NMR $(500 \mathrm{MHz}$, $\left.\mathrm{CDCl}_{3}\right) \delta 7.79$ (s. $\left.2 \mathrm{H}\right), 7.70$ (s. $\left.1 \mathrm{H}\right), 7.61-7.57$ (m, 2H), 7.53-7.50 (m, 2H), 7.43-7.39 (td, 2H, $J=3.5$ y $J=7.6 \mathrm{~Hz}), 4.94-4.88$ (m, 2H), 3.5 (d, 3H, $J=11.3 \mathrm{~Hz}), 2.86-2.78$ (m, 1H), 2.41-2.34 $(\mathrm{m}, 1 \mathrm{H}), 1.22$ (s, 9H) ppm; ${ }^{13} \mathrm{C}$ NMR $\left(125 \mathrm{MHz}, \mathrm{CDCl}_{3}\right) \delta 143.9\left(\mathrm{~d}, J_{\mathrm{CP}}=8.2 \mathrm{~Hz}\right), 133.1(\mathrm{~d}$, $\left.J_{\mathrm{CP}}=2.5 \mathrm{~Hz}\right), 132.3,132,129.6\left(\mathrm{~d}, J_{\mathrm{CP}}=126.5 \mathrm{~Hz}\right), 129.2\left(\mathrm{~d}, J_{\mathrm{CP}}=12.8 \mathrm{~Hz}\right), 128.2\left(\mathrm{~d}, J_{\mathrm{CP}}=\right.$ $2.3 \mathrm{~Hz}), 124.5,122.4(\mathrm{bs}), 59.6,54.6,51.5\left(\mathrm{~d}, J_{\mathrm{CP}}=6.4 \mathrm{~Hz}\right), 37.1\left(\mathrm{~d}, J_{\mathrm{CP}}=99 \mathrm{~Hz}\right), 22.8 \mathrm{ppm}$; ${ }^{31} \mathrm{P}$ NMR $\left(202 \mathrm{MHz}, \mathrm{CDCl}_{3}\right) \delta+42.1$ ppm; IR 3212, 3050, 2922, 1375, 1283, 1207, 1178, 1126, 1078, 1025, 899, 788, 743, $707 \mathrm{~cm}^{-1}$; HRMS: calcd for $\mathrm{C}_{21} \mathrm{H}_{25} \mathrm{~F}_{6} \mathrm{NO}_{3} \mathrm{PS}[\mathrm{M}+1]$ : 516.1197; found: 516.1196 .

Methyl 2-(tert-butylsulfinamide)-2-(2'-naphthyl)ethyl phenylphosphinate, $10(R, S, R)$ and $10(R, S, S)$.

It was prepared following the general procedure, starting from phosphinate rac-7 $(98 \mathrm{mg}$, $0.57 \mathrm{mmol})$, a $1 \mathrm{M}$ solution of LHMDS in THF $(1.73 \mathrm{~mL}, 1.73 \mathrm{mmol})$ and $N$-sulfinylimine 
$\mathbf{4 R},(150 \mathrm{mg}, 0.57 \mathrm{mmol})$. The resulting residue was obtained as phosphinates $\mathbf{1 0}(\boldsymbol{R}, \boldsymbol{S}, \boldsymbol{R})$ and $\mathbf{1 0}(R, S, S)$ in a 1:1 ratio. Purification by column chromatography (EtOAc: $\left.\mathrm{CH}_{2} \mathrm{Cl}_{2}, 1: 1\right)$ gave both diastereomerically pure phosphinates $\mathbf{1 0}(\boldsymbol{R}, \boldsymbol{S}, \boldsymbol{R})(110 \mathrm{mg}, 45 \%)$ and $\mathbf{1 0}(\boldsymbol{R}, \boldsymbol{S}, \boldsymbol{S})(120 \mathrm{mg}$, $49 \%$ ) as white solids.

Diastereomer 10(R,S,R), SulPhos: mp $168^{\circ} \mathrm{C} ;[\alpha]_{\mathrm{D}}{ }^{20}=+13.2\left(c 0.5\right.$ in $\left.\mathrm{CHCl}_{3}\right)$; ${ }^{1} \mathrm{H}$ NMR $\left(500 \mathrm{MHz}, \mathrm{CDCl}_{3}\right) \delta 7.80-7.71(\mathrm{~m}, 4 \mathrm{H}), 7.60-7.57(\mathrm{~m}, 2 \mathrm{H}), 7.46-7.39(\mathrm{~m}, 4 \mathrm{H}), 7.32-7.28(\mathrm{~m}$, 2H), $5.13(\mathrm{~d}, 1 \mathrm{H}, J=4.2 \mathrm{~Hz}), 5.05(\mathrm{~m}, 1 \mathrm{H}), 3.55(\mathrm{~d}, 3 \mathrm{H}, J=11.1 \mathrm{~Hz}), 2.74(\mathrm{~m}, 1 \mathrm{H}), 2.75(\mathrm{td}$, $1 \mathrm{H}, J=4.8 \mathrm{~Hz}$ and $15.4 \mathrm{~Hz}), 1.23(\mathrm{~s}, 9 \mathrm{H}) \mathrm{ppm} ;{ }^{13} \mathrm{C} \mathrm{NMR}\left(125 \mathrm{MHz}, \mathrm{CDCl}_{3}\right) \delta 138\left(\mathrm{~d}, J_{\mathrm{CP}}=\right.$ $9.2 \mathrm{~Hz}), 133.1\left(\mathrm{~d}, J_{\mathrm{CP}}=8.6 \mathrm{~Hz}\right), 132.3\left(\mathrm{~d}, J_{\mathrm{CP}}=2.6 \mathrm{~Hz}\right), 131.4\left(\mathrm{~d}, J_{\mathrm{CP}}=10.2 \mathrm{~Hz}\right), 129.9(\mathrm{~d}$, $\left.J_{\mathrm{CP}}=125.9 \mathrm{~Hz}\right), 128.6,128.5,128.1,127.5,126.5,126.2,126.1,125,56.3,54.9\left(\mathrm{~d}, J_{\mathrm{CP}}=2.9\right.$ $\mathrm{Hz}), 51.1\left(\mathrm{~d}, J_{\mathrm{CP}}=6.4 \mathrm{~Hz}\right), 37.3\left(\mathrm{~d}, J_{\mathrm{CP}}=98.8 \mathrm{~Hz}\right), 22.5 \mathrm{ppm} ;{ }^{31} \mathrm{P} \mathrm{NMR}\left(202 \mathrm{MHz}, \mathrm{CDCl}_{3}\right)$ $\delta+43.4$ ppm; IR 3212, 2948, 2922, 1439, 1360, 1212, 1120, 1074, 1036, 1025, 834, 819, 756, 741, $693 \mathrm{~cm}^{-1}$; EA: calcd for: C, 64.32\%; H, 6.57\%. N, 3.26\%; S, 7.47\%; found: C, 63.66\%; H, 6.68\%. N, 3.87\%; S, 7.38\%; HRMS: calcd for $\mathrm{C}_{23} \mathrm{H}_{29} \mathrm{NO}_{3} \mathrm{PS}[\mathrm{M}+\mathrm{H}]: 430.1606$; found: 430.1616; MS: 372, 310, 309, 156, 155 (100\%), 154, 140, 91, 77, 60, 57.

Diastereoisomer 10(R,S,S): mp $140-141^{\circ} \mathrm{C} ;[\alpha]_{\mathrm{D}}{ }^{20}=-36.7\left(\right.$ c 1.0 in $\left.\mathrm{CHCl}_{3}\right) ;{ }^{1} \mathrm{H}$ NMR (500 $\left.\mathrm{MHz} \mathrm{CDCl}_{3}\right) \delta 7.77-7.74(\mathrm{~m}, 3 \mathrm{H}), 7.71(\mathrm{~d}, 1 \mathrm{H}, J=8.5 \mathrm{~Hz}), 7.62-7.58(\mathrm{~m}, 2 \mathrm{H}), 7.46-7.39(\mathrm{~m}$, $4 \mathrm{H}), 7.31(\mathrm{td}, 2 \mathrm{H}, J=3.4$ y $7.7 \mathrm{~Hz}), 4.87(\mathrm{~m}, 1 \mathrm{H}), 4.79(\mathrm{~d}, 1 \mathrm{H}, J=3.4 \mathrm{~Hz}),, 3.56\left(\mathrm{~d}, 3 \mathrm{H}, J_{\mathrm{HP}}=\right.$ $11.2 \mathrm{~Hz}$ ), 2.94 (ddd, $1 \mathrm{H}, J=8.2,15.1$ y $17.4 \mathrm{~Hz}$ ), 2.45 (ddd, $1 \mathrm{H}, J=5.6,11.2$ y $16.8 \mathrm{~Hz}$ ), $1.22(\mathrm{~s}, 9 \mathrm{H}) \mathrm{ppm} ;{ }^{13} \mathrm{C} \mathrm{NMR}\left(125 \mathrm{MHz}, \mathrm{CDCl}_{3}\right) \delta 137.8\left(\mathrm{~d}, J_{\mathrm{CP}}=9.9 \mathrm{~Hz}\right), 133.1\left(\mathrm{~d}, J_{\mathrm{CP}}=4.5\right.$ $\mathrm{Hz}), 132.3\left(\mathrm{~d}, J_{\mathrm{CP}}=2.5 \mathrm{~Hz}\right), 131.5\left(\mathrm{~d}, J_{\mathrm{CP}}=10 \mathrm{~Hz}\right), 129.60\left(\mathrm{~d}, J_{\mathrm{CP}}=126.3 \mathrm{~Hz}\right), 128.6,128.5$, $128.5,128.1,127.5,126.7,126.2,125.1,56.2,54.8,51.1\left(\mathrm{~d}, J_{\mathrm{CP}}=6.4 \mathrm{~Hz}\right), 36.9$ (d, $J_{\mathrm{CP}}=98.4$ $\mathrm{Hz}), 22.5$ ppm; ${ }^{31} \mathrm{P}$ NMR (202 MHz, $\left.\mathrm{CDCl}_{3}\right) \delta+43.2$ ppm; IR 3212, 2948, 2922, 1439, 1360, 1212, 1120, 1074, 1036, 1025, 834, 819, 756, 741, $693 \mathrm{~cm}^{-1}$; EA: calcd for: C, 64.32\%; H, 6.57\%. N, 3.26\%; S, 7.47\%; found: C, 63.66\%; H, 6.68\%. N, 3.87\%; S, 7.38\%; HRMS: calcd for $\mathrm{C}_{23} \mathrm{H}_{29} \mathrm{NO}_{3} \mathrm{PS}$ : 430.1606; found: 430.1616; MS: 372, 310, 309, 156, 155 (100\%), 154, $140,91,77,60,57,55$. 
Methyl 2-(tert-butylsulfinamide)-2-(ferrocenyl)ethyl phenylphosphinate, $11(R, S, R)$ and $11(R, S, S)$.

It was prepared following the general procedure, starting from phosphinate rac -7 $(559.79 \mathrm{mg}$, $3.29 \mathrm{mmol})$, a $1 \mathrm{M}$ solution of LHMDS in THF $(9.87 \mathrm{~mL}, 9.87 \mathrm{mmol})$ and $N$-sulfinylimine $\mathbf{5} \boldsymbol{R}$, $(1.04 \mathrm{~g}, 3.29 \mathrm{mmol})$. The resulting residue was obtained as phosphinates $11(\boldsymbol{R}, \boldsymbol{S}, \boldsymbol{R})$ and $\mathbf{1 1}(\boldsymbol{R}, S, S)$ in a 1:1 ratio. Purification by crystallization (EtOAc:ether, 2:1) gave diastereomerically pure phosphinate $\mathbf{1 1}(\boldsymbol{R}, S, \boldsymbol{R})(753.64 \mathrm{mg}, 47 \%)$ as yelow solid: $\mathrm{mp} 120^{\circ} \mathrm{C}$; $[\alpha]_{\mathrm{D}}{ }^{20}=-48.6\left(c 0.5\right.$ in $\left.\mathrm{CH}_{2} \mathrm{Cl}_{2}\right) ;{ }^{1} \mathrm{H}$ NMR $\left(500 \mathrm{MHz}, \mathrm{CDCl}_{3}\right) \delta 7.78-7.72(\mathrm{~m}, 2 \mathrm{H}), 7.58-7.54$ (m, $1 \mathrm{H}), 7.51-7.47(\mathrm{~m}, 2 \mathrm{H}), 4.72-4.66(\mathrm{~m}, 1 \mathrm{H}), 4.5(\mathrm{~d}, 1 \mathrm{H}, J=3.7 \mathrm{~Hz}), 4.26(\mathrm{t}, 2 \mathrm{H}, J=1.8$ $\mathrm{Hz}), 4.16(\mathrm{~s}, 5 \mathrm{H}), 4.15(\mathrm{t}, 1 \mathrm{H}, J=2.2 \mathrm{~Hz}), 4.12$ (c, 1H, $J=7 \mathrm{~Hz}), 3.6$ (d, 3H, $J=11.1 \mathrm{~Hz}), 2.73-$ $2.54(\mathrm{~m}, 2 \mathrm{H}), 1.18(\mathrm{~s}, 9 \mathrm{H}) \mathrm{ppm} ;{ }^{13} \mathrm{C} \mathrm{NMR}\left(125 \mathrm{MHz}, \mathrm{CDCl}_{3}\right) \delta 132.8\left(\mathrm{~d}, J_{\mathrm{CP}}=2.5 \mathrm{~Hz}\right), 132$ $\left(\mathrm{d}, J_{\mathrm{CP}}=10.1 \mathrm{~Hz}\right), 130.7\left(\mathrm{~d}, J_{\mathrm{CP}}=124.8 \mathrm{~Hz}\right), 129.1\left(\mathrm{~d}, J_{\mathrm{CP}}=14.6 \mathrm{~Hz}\right), 89.9\left(\mathrm{~d}, J_{\mathrm{CP}}=11.3 \mathrm{~Hz}\right)$ $89.8,69.5,69,68.7,68.2\left(\mathrm{~d}, J_{\mathrm{CP}}=28 \mathrm{~Hz}\right), 68.1,55.9,51.4\left(\mathrm{~d}, J_{\mathrm{CP}}=6.4 \mathrm{~Hz}\right), 50.8\left(\mathrm{~d}, J_{\mathrm{CP}}=2.4\right.$ $\mathrm{Hz}), 37.15\left(\mathrm{~d}, J_{\mathrm{CP}}=100.5 \mathrm{~Hz}\right), 22.9 \mathrm{ppm} ;{ }^{31} \mathrm{P} \mathrm{NMR}\left(202 \mathrm{MHz}, \mathrm{CDCl}_{3}\right) \delta+43.8 \mathrm{ppm}$; IR 3463, 2951, 2923, 1438, 1213, 1120, 1032, 819, 731, 697, $634 \mathrm{~cm}^{-1}$; HRMS: calcd for $\mathrm{C}_{23} \mathrm{H}_{30} \mathrm{FeNO}_{3} \mathrm{PS}$ : 487.1033; found: 487.1026.

Methyl 2-(tert-butylsulfinamide)-2-(isopropyl)ethyl phenylphosphinate, $12(R, S, R)$ and $12(R, S, S)$.

It was prepared following the general procedure, starting from phosphinate rac -7 $(867.77 \mathrm{~g}$, $5.1 \mathrm{mmol})$, a $1 \mathrm{M}$ solution of LHMDS in THF $(15.3 \mathrm{~mL}, 15.3 \mathrm{mmol})$ and $N$-sulfinylimine $\mathbf{6} \boldsymbol{R}$, (890 mg, $5.1 \mathrm{mmol})$. The resulting residue was obtained as phosphinates $\mathbf{1 2}(\boldsymbol{R}, \boldsymbol{S}, \boldsymbol{R})$ and $\mathbf{1 2}(\boldsymbol{R}, S, S)$ in a 1:1 ratio. Purification by column chromatography (EtOAc:ether, 4:1) gave diastereomerically pure phosphinate $\mathbf{1 2}(\boldsymbol{R}, S, \boldsymbol{R})(775.17 \mathrm{mg}, 44 \%)$ as a white solid: $\mathrm{mp} 127^{\circ} \mathrm{C}$; $[\alpha]_{\mathrm{D}}{ }^{20}=+6.2\left(c 1.0\right.$ in $\left.\mathrm{CHCl}_{3}\right) ;{ }^{1} \mathrm{H}$ NMR $\left(500 \mathrm{MHz}, \mathrm{CDCl}_{3}\right) \delta$ 7.78-7.74 (m, 2H). 7.57-7.55 (m, 1H), 7.51-7.48 (m, 2H), $4.03(\mathrm{~d}, 1 \mathrm{H}, J=6.8 \mathrm{~Hz}), 3.58(\mathrm{~d}, 3 \mathrm{H}, J=11.1 \mathrm{~Hz}), 3.53(\mathrm{~m}, 1 \mathrm{H})$, 2.26-2.22 (m, 1H), 2.16-2.12 (m, 2H), 1.17 (s, 9H), 0.94 (d, 3H, J=6.8 Hz), 0.90 (d, 3H, J= $6.8 \mathrm{~Hz}) \mathrm{ppm} ;{ }^{13} \mathrm{C} \mathrm{NMR}\left(125 \mathrm{MHz}, \mathrm{CDCl}_{3}\right) \delta 132.6\left(\mathrm{~d}, J_{\mathrm{CP}}=2.6 \mathrm{~Hz}\right), 131.7\left(\mathrm{~d}, J_{\mathrm{CP}}=10.0 \mathrm{~Hz}\right)$, $130.7\left(\mathrm{~d}, J_{\mathrm{CP}}=123.9 \mathrm{~Hz}\right), 129\left(\mathrm{~d}, J_{\mathrm{CP}}=12.7 \mathrm{~Hz}\right), 57.9\left(\mathrm{~d}, J_{\mathrm{CP}}=3.6 \mathrm{~Hz}\right), 56.2,51.1\left(\mathrm{~d}, J_{\mathrm{CP}}=6.4\right.$ $\mathrm{Hz}$ ), 32.4, 32.4, 32 (d, $\left.J_{\mathrm{CP}}=91 \mathrm{~Hz}\right), 22.6,18.9,17.6 \mathrm{ppm} ;{ }^{31} \mathrm{P} \mathrm{NMR}\left(202 \mathrm{MHz}, \mathrm{CDCl}_{3}\right)$ $\delta+44.6$ ppm; IR 3217, 2957, 1461, 1438, 1390, 1362, 1213, 1177, 1120, 1060, 1038, 1013, 766, $751 \mathrm{~cm}^{-1}$; HRMS: calcd for $\mathrm{C}_{16} \mathrm{H}_{28} \mathrm{NO}_{3} \mathrm{NaPS}$ : 368.1425; found: 368.1419 . 
(R,S)-2-(tert-Butilsulfinamide)-2-(2'-naphthyl)ethyl diphenyl phosphine oxide, 17(R,S).

A $1 \mathrm{M}$ solution of LHMDS in THF $(11.5 \mathrm{~mL}, 11.5 \mathrm{mmol})$ was added to a solution of diphenyl(methyl)phosphine oxide $15(0.99 \mathrm{~g}, 4.64 \mathrm{mmol})$ in THF (20 mL) under argon atmosphere at $-78^{\circ} \mathrm{C}$. The reaction mixture was stirred at $-78^{\circ} \mathrm{C}$ for $2 \mathrm{~h}$ in order to obtain the corresponding anion. Then, this solution was added via cannula to a solution of $\mathrm{N}$ sulfinylimine $\mathbf{4 R}$, (1 g, $3.84 \mathrm{mmol})$ in THF $(10 \mathrm{~mL})$, previously cooled to $-78^{\circ} \mathrm{C}$. After stirring for $2 \mathrm{~h}$ at $-78^{\circ} \mathrm{C}$, the reaction mixture was hydrolized with a saturated $\mathrm{NH}_{4} \mathrm{Cl}$ aqueous solution $(30 \mathrm{~mL})$. The aqueous phase was then extracted with EtOAc $(5 \mathrm{x} 40 \mathrm{~mL})$ and the combined organic phases were dried with anhydrous $\mathrm{Na}_{2} \mathrm{SO}_{4}$. The solvent was evaporated under reduced pressure and the crude was purified by flash chromatography (EtOH:ether, 1:10) obtaining compound $\mathbf{1 7}(\boldsymbol{R}, S)(1.7 \mathrm{~g}, 95 \%)$ as a white solid: $\mathrm{mp} 216^{\circ} \mathrm{C} ;[\alpha]_{\mathrm{D}}{ }^{20}=-15.9(c$ 1.0 in $\left.\mathrm{CHCl}_{3}\right)$; ${ }^{1} \mathrm{H}$ NMR (500 MHz, $\left.\mathrm{CDCl}_{3}\right) \delta 7.78-7.63(\mathrm{~m}, 8 \mathrm{H}), 7.46-7.35(\mathrm{~m}, 9 \mathrm{H}), 5.62(\mathrm{~d}$, $1 \mathrm{H}, J=3.6 \mathrm{~Hz}$ ), 4.97 (ddd, 1H, $J=3.7,8.5$ and $16.3 \mathrm{~Hz}$ ), 3.22 (ddd, $1 \mathrm{H}, J=9.0,11.5$ and 15.0 $\mathrm{Hz}), 2.80$ (ddd, $1 \mathrm{H}, J=4.0,8.4$ and $15.0 \mathrm{~Hz}), 1.18$ (s, 9H) ppm; ${ }^{13} \mathrm{C} \mathrm{NMR}\left(125 \mathrm{MHz}, \mathrm{CDCl}_{3}\right)$ $\delta 138.0(\mathrm{~d}, J=9.9 \mathrm{~Hz}), 133.5,133.1,132.7,132.4,131.8(\mathrm{~d}, J=2 \mathrm{~Hz}), 131$ (d, $J=2.6 \mathrm{~Hz})$, $131.6,130.8,130.8,130.5,130.4,128.7,128.7,128.6,128.6,128.6,128.1,127.6,126.7$, $126.1(\mathrm{~d}, J=1.5 \mathrm{~Hz}), 125.2,56.3,55.6(\mathrm{~d}, J=3.0 \mathrm{~Hz}), 37.3(\mathrm{~d}, J=69.2 \mathrm{~Hz}), 22.5 \mathrm{ppm} ;{ }^{31} \mathrm{P}$ NMR (202 MHz, $\left.\mathrm{CDCl}_{3}\right) \delta+30.9$ ppm; IR 3208, 1437, 1172, 1071, 1052, 902, 864, $817 \mathrm{~cm}^{-1}$; HRMS: calcd for $\mathrm{C}_{28} \mathrm{H}_{30} \mathrm{NO}_{2} \mathrm{SPNa}[\mathrm{M}+\mathrm{Na}]^{+}$: 498.1633; found: 498.1647.

\section{(R,S)-Dimethyl 2-(tert-butilsulfinamide)-2-(2'-naphthyl)ethylfosfonate, $18(R, S)$.}

A 1M solution of LHMDS in THF $(11.5 \mathrm{~mL}, 11.5 \mathrm{mmol})$ was added to a solution of dimethyl phenyl methylphosphonate 16 (577 mg,4.65 mmol) in THF (20mL) under argon atmosphere at $-78^{\circ} \mathrm{C}$. The reaction mixture was stirred at $-78^{\circ} \mathrm{C}$ for $2 \mathrm{~h}$ in order to obtain the corresponding anion. Then, this solution was added via cannula to a solution of $N$-sulfinylimine $\mathbf{4 R},(1 \mathrm{~g}$, $3.84 \mathrm{mmol})$ in THF $(10 \mathrm{~mL})$, previously cooled to $-78^{\circ} \mathrm{C}$. After stirring for $2 \mathrm{~h}$ at $-78^{\circ} \mathrm{C}$, the reaction mixture was hydrolized with a saturated $\mathrm{NH}_{4} \mathrm{Cl}$ aqueous solution $(30 \mathrm{~mL})$. The aqueous phase was then extracted with EtOAc $(5 \times 40 \mathrm{~mL})$ and the combined organic phases were dried with anhydrous $\mathrm{Na}_{2} \mathrm{SO}_{4}$. The solvent was evaporated under reduced pressure and the crude was purified by flash chromatography (EtOH:ether, 1:10) obtaining $1.4 \mathrm{~g}$ of compound $\mathbf{1 8}(\boldsymbol{R}, \boldsymbol{S})(1.43 \mathrm{~g}, 97 \%)$ as a white solid: $\mathrm{mp} 131^{\circ} \mathrm{C} ;[\alpha]_{\mathrm{D}}{ }^{20}=-30.2\left(c 0.5\right.$ in $\left.\mathrm{CHCl}_{3}\right)$; ${ }^{1} \mathrm{H}$ NMR (500 MHz, $\left.\mathrm{CDCl}_{3}\right) \delta$ 7.87-7.81 (m, 4H), 7.51-7.47 (m, 3H), 4.97-4.90 (m, 1H), 4.60 $(\mathrm{d}, 2 \mathrm{H}, J=3.9 \mathrm{~Hz}), 3.60(\mathrm{~d}, 3 \mathrm{H}, J=11.0 \mathrm{~Hz}), 3.54(\mathrm{~d}, 3 \mathrm{H}, J=10.9 \mathrm{~Hz}), 2.6$ (ddd, $1 \mathrm{H}, J=7.6$, 
15.3 and $17.9 \mathrm{~Hz}), 2.39$ (ddd, $1 \mathrm{H}, J=5.9,15.3$ and $18.2 \mathrm{~Hz}), 1.21(\mathrm{~s}, 9 \mathrm{H}) \mathrm{ppm} ;{ }^{13} \mathrm{C} \mathrm{NMR}$ $\left(125 \mathrm{MHz} \mathrm{CDCl}_{3}\right) \delta 138.1(\mathrm{~d}, J=9.8 \mathrm{~Hz}), 133.2(\mathrm{~d}, J=9.2 \mathrm{~Hz}), 128.26,128.2,127.7,126.5$, 126.4, 126.3, 125.1, 56.2, 54.7 (d, $J=1.7 \mathrm{~Hz}), 52.4$ (d, $J=6.4 \mathrm{~Hz}), 52.2$ (d, $J=6.5 \mathrm{~Hz}), 32.8$ (d, $J=139.2 \mathrm{~Hz}), 29.7,22.5$ ppm; ${ }^{31} \mathrm{P}$ NMR (202 MHz, $\left.\mathrm{CDCl}_{3}\right) \delta+30.2 \mathrm{ppm}$; IR 3186, 1240 , 1224, 1182, 1069, 1035, 1015, 861, 748, $660 \mathrm{~cm}^{-1}$; HRMS: $\mathrm{C}_{18} \mathrm{H}_{26} \mathrm{NO}_{4} \mathrm{NaSP}$ : calcd for 406.1228; found: 406.1218 .

(S,R)-Methyl 2-(tert-butylsulfonamide)-2-(2'-naphthyl)ethylphenylphosphinate, $19(S, R)$.

It was prepared following the general procedure from SulPhos $\mathbf{1 0}(\boldsymbol{R}, \boldsymbol{S}, \boldsymbol{R})(170 \mathrm{mg}, 0.39$ mmol), $\mathrm{NaIO}_{4}(722 \mathrm{mg}, 5.83 \mathrm{mmol})$ and $\mathrm{RuCl}_{3} \cdot \mathrm{H}_{2} \mathrm{O}(1 \mathrm{~mol} \%)$. The resulting residue was purified by flash chromatography (EtOAc:hexane 1:1) obtaining optically pure compound $\mathbf{1 9}(\boldsymbol{S}, \boldsymbol{R})(161 \mathrm{mg}, 93 \%)$ as a white solid: $\mathrm{mp} 132^{\circ} \mathrm{C} ;[\alpha]_{\mathrm{D}}{ }^{20}=-19.8\left(c 0.5\right.$ in $\left.\mathrm{CHCl}_{3}\right) ;{ }^{1} \mathrm{H} \mathrm{NMR}$ $\left(500 \mathrm{MHz} \mathrm{CDCl}_{3}\right)$ 87.74-7.71 (m, 2H), $7.64(\mathrm{~d}, 1 \mathrm{H}, J=7.5 \mathrm{~Hz}), 7.50(\mathrm{~d}, 1 \mathrm{H}, J=8.4 \mathrm{~Hz})$, 7.44-7.39 (m, 2H), 7.29-7.25 (m, 3H), $7.16(\mathrm{dd}, 1 \mathrm{H}, J=1.3$ and $8.4 \mathrm{~Hz}), 7.12-7.11(\mathrm{~m}, 1 \mathrm{H})$, $6.96(\mathrm{td}, 2 \mathrm{H}, J=3.4$ and $6.1 \mathrm{~Hz}), 4.87(\mathrm{~m}, 1 \mathrm{H}), 6.47(\mathrm{~d}, 1 \mathrm{H}, J=8.5 \mathrm{~Hz}), 5.23(\mathrm{dq}, 1 \mathrm{H}, J=3.5$ and $28.3 \mathrm{~Hz}), 3.49(\mathrm{~d}, 3 \mathrm{H}, J=11.1 \mathrm{~Hz}), 2.74(\mathrm{td}, 1 \mathrm{H}, J=5.0$ y $15.7 \mathrm{~Hz}), 2.62(\mathrm{ddd}, 1 \mathrm{H}, J=$ $5.7 \mathrm{~Hz}, 10.9 \mathrm{~Hz}$ and $15.5 \mathrm{~Hz}), 1.22$ (s, 9H) ppm; ${ }^{13} \mathrm{C} \mathrm{NMR}\left(125 \mathrm{MHz}, \mathrm{CDCl}_{3}\right) \delta 136.5(\mathrm{~d}$, $\left.J_{\mathrm{CP}}=5.3 \mathrm{~Hz}\right), 131.9,131.6,130.6\left(\mathrm{~d}, J_{\mathrm{CP}}=2.7 \mathrm{~Hz}\right), 130.1\left(\mathrm{~d}, J_{\mathrm{CP}}=10.3 \mathrm{~Hz}\right), 128.4\left(\mathrm{~d}, J_{\mathrm{CP}}=\right.$ $126.5 \mathrm{~Hz}), 127.2,127.0$ (d, $\left.J_{\mathrm{CP}}=6.4 \mathrm{~Hz}\right), 126.9,126.3,125.1,124.9,124.4,123.0,58.0,52.8$ $\left(\mathrm{d}, J_{\mathrm{CP}}=3.9 \mathrm{~Hz}\right), 50.9\left(\mathrm{~d}, J_{\mathrm{CP}}=6.4 \mathrm{~Hz}\right), 36.5\left(\mathrm{~d}, J_{\mathrm{CP}}=98.5 \mathrm{~Hz}\right), 23.1 \mathrm{ppm} ;{ }^{31} \mathrm{P}$ NMR $(202$ $\left.\mathrm{MHz}, \mathrm{CDCl}_{3}\right) \delta+43.7$ ppm; IR 3177, 1478, 1298, 1209, 1126, 1041, 1013, 935, 748, $697 \mathrm{~cm}^{-}$ ${ }^{1}$; HRMS: $\mathrm{C}_{23} \mathrm{H}_{28} \mathrm{NO}_{4} \mathrm{PS}$ : calcd for 445.1477; found: 445.1469.

\section{$N$-arylimines. General method.}

A mixture of corresponding ketone (10 mmol, 1equiv.), corresponding amine (aniline or $p$ methoxianiline) (10 mmol, 1 equiv.) and molecular sieve $4 \AA$ ( $1 \mathrm{~g} / \mathrm{mmol})$ at $80^{\circ} \mathrm{C}$, under argon atmosphere, in dry toluene $(15 \mathrm{~mL})$ was stirred during $24 \mathrm{~h}$. The resulting suspension was filtered through a pad of Celite and the solvent was evaporated under reduced pressure. The obtained crude was purified by flash chromatography or crystallization to obtain the desired $N$-arylimine. 


\section{$N$-(1-Phenylethylidene)aniline, 13.}

It was prepared following the general procedure from acetophenone $(1.16 \mathrm{~mL}, 10 \mathrm{mmol})$ and aniline $(0.91 \mathrm{~mL}, 10 \mathrm{mmol})$. The resulting residue was purified by crystallization from EtOH to give compound 13 (1.56 g, 80\%) as a yellow solid: mp $40-41{ }^{\circ} \mathrm{C} ;{ }^{1} \mathrm{H}$ NMR $(500 \mathrm{MHz}$, $\left.\mathrm{CDCl}_{3}\right) \delta 7.99(\mathrm{dd}, 2 \mathrm{H}, J=1.5$ and $7.2 \mathrm{~Hz}), 7.48-7.43(\mathrm{~m}, 3 \mathrm{H}), 7.35(\mathrm{t}, 2 \mathrm{H}, J=7.7 \mathrm{~Hz}), 7.09$ $(\mathrm{t}, 1 \mathrm{H}, J=7.4 \mathrm{~Hz}), 6.80(\mathrm{~d}, 2 \mathrm{H}, J=7.5 \mathrm{~Hz}), 2.24(\mathrm{~s}, 3 \mathrm{H}) \mathrm{ppm} ;{ }^{13} \mathrm{C} \mathrm{NMR}\left(125 \mathrm{MHz}, \mathrm{CDCl}_{3}\right)$ $\delta 165.4,151.7,139.5,130.4,128.9,128.5,128.34, \quad 128.0,127.7,127.1,123.1,119.3,17.3$ ppm; HRMS: calcd for $\mathrm{C}_{14} \mathrm{H}_{13} \mathrm{~N}$ [M+ H]: 195.1048; found: 195.1056 .

\section{$N$-(1-Phenylethylidene)-p-methoxyaniline, 20.}

It was prepared following the general procedure from acetophenone $(1.16 \mathrm{~mL}, 10 \mathrm{mmol})$ and $p$-methoxyaniline $(1.23 \mathrm{~g}, 10 \mathrm{mmol})$. The resulting residue was purified by crystallization from $\mathrm{EtOH}$ to give compound $20(2.07 \mathrm{~g}, 92 \%)$ as a yellow solid: mp $85{ }^{\circ} \mathrm{C}$; ${ }^{1} \mathrm{H}$ NMR (300 $\left.\mathrm{MHz}, \mathrm{CDCl}_{3}\right) \delta 7.98-7.93(\mathrm{~m}, 2 \mathrm{H}), 7.47-7.40(\mathrm{~m}, 3 \mathrm{H}), 6.90(\mathrm{~d}, 2 \mathrm{H}, J=8.9 \mathrm{~Hz}), 6.75(\mathrm{~d}, 2 \mathrm{H}$, $J=8.9 \mathrm{~Hz}), 3.81(\mathrm{~s}, 3 \mathrm{H}), 2.25$ (s, 3H) ppm; ${ }^{13} \mathrm{C} \mathrm{NMR}\left(75 \mathrm{MHz}, \mathrm{CDCl}_{3}\right) \delta 165.7,155.9,144.8$, $139.8,130.3,128.3,127.1,120.7,114.2,55.5,17.3 \mathrm{ppm}$.

\section{$N$-1-(p-Methoxyphenyl)ethylidene]aniline, 21.}

It was prepared following the general procedure from $p$-methoxyacetophenone $(1.50 \mathrm{~g}, 10$ mmol) and aniline $(0.91 \mathrm{~mL}, 10 \mathrm{mmol})$. The resulting residue was purified by flash chromatography (EtOAc:hexane, 1:5) to give compound 21 (1.96 g, 87\%) as a white solid: ${ }^{1} \mathrm{H}$ NMR $\left(300 \mathrm{MHz}, \mathrm{CDCl}_{3}\right.$ ) $87,97(\mathrm{dt}, 2 \mathrm{H}, J=3.1$ and $8.9 \mathrm{~Hz}), 7.39-7.33(\mathrm{~m}, 2 \mathrm{H}), 7,09$ (tt, $1 \mathrm{H}, J=1.5$ and $7.4 \mathrm{~Hz}), 6,97(\mathrm{dt}, 2 \mathrm{H}, J=2.7$ and $8.9 \mathrm{~Hz}), 6.81(\mathrm{dd}, 2 \mathrm{H}, J=6.1$ and $8.4 \mathrm{~Hz})$, 3.89 (s, 3H), 2.25 (s, 3H) ppm; ${ }^{13} \mathrm{C}$ NMR (75 MHz, $\left.\mathrm{CDCl}_{3}\right) \delta 164.6,161.6,152.0,132.3$, $128.04,128.9,123.1,119.7,113.7,55.5,17.2 \mathrm{ppm}$.

\section{$N$-[1-(p-Methoxyphenyl)ethylidene]-p-methoxyaniline, 22.}

It was prepared following the general procedure from $p$-methoxyacetophenone $(1.50 \mathrm{~g}, 10$ mmol) and $p$-methoxyaniline $(1.23 \mathrm{~g}, 10 \mathrm{mmol})$. The resulting residue was purified by flash chromatography (toluene) to give compound $22(2.30 \mathrm{~g}, 90 \%)$ as a yellow solid: $\mathrm{mp}$ 133-134 ${ }^{\circ} \mathrm{C} ;{ }^{1} \mathrm{H}$ NMR $\left(300 \mathrm{MHz}, \mathrm{CDCl}_{3}\right) \delta 7.93(\mathrm{~d}, 2 \mathrm{H}, J=8.9 \mathrm{~Hz}), 6.96(\mathrm{~d}, 2 \mathrm{H}, J=8.9 \mathrm{~Hz}), 6.92(\mathrm{~d}$, $2 \mathrm{H}, J=8.9 \mathrm{~Hz}), 6.74(\mathrm{~d}, 2 \mathrm{H}, J=8.9 \mathrm{~Hz}), 3.86(\mathrm{~s}, 3 \mathrm{H}), 3.81(\mathrm{~s}, 3 \mathrm{H}), 2.21(\mathrm{~s}, 3 \mathrm{H}) \mathrm{ppm} ;{ }^{13} \mathrm{C} \mathrm{NMR}$ 
$\left(75 \mathrm{MHz}, \mathrm{CDCl}_{3}\right) \delta 164.8,161.4,155.8,145.1,132.5,128.7,120.9,114.2,113.6,55.5,55.4$, $17.1 \mathrm{ppm}$.

\section{$N$-[1-(3'-Methoxyphenyl)ethylidene]-p-methoxyaniline, 23.}

It was prepared following the general procedure from 3-methoxyacetophenone $(1.50 \mathrm{~g}, 10$ mmol) and $p$-methoxyaniline $(1.23 \mathrm{~g}, 10 \mathrm{mmol})$. The resulting residue was purified by flash chromatography (toluene) to give compound $23(2.48 \mathrm{~g}, 97 \%)$ as a yellow solid: ${ }^{1} \mathrm{H}$ NMR $\left(300 \mathrm{MHz}, \mathrm{CDCl}_{3}\right) \delta 7.59-7.56(\mathrm{~m}, 1 \mathrm{H}), 7.51-7.49(\mathrm{~m}, 1 \mathrm{H}), 7.35(\mathrm{t}, 1 \mathrm{H}, J=8.0 \mathrm{~Hz}), 7.03-7.0$ (m, 1H), 6.94-6.90 (m, 2H), 6.79-6.74 (m, 2H), 3.88 (s, 3H), $3.82(\mathrm{~s}, 3 \mathrm{H}), 2.24$ (s, 3H) ppm; ${ }^{13} \mathrm{C}$ NMR $\left(100 \mathrm{MHz}, \mathrm{CDCl}_{3}\right) \delta 165.4,159.5,155.8,144.6,141.1,129.2,120.6,119.7,116.6$, 114.2, 111.7, 55.5, 55.4, 17.5 ppm; EA: calcd for: $\mathrm{C}_{16} \mathrm{H}_{17} \mathrm{NO}_{2}$ : C, 75.27; H, 6.71; N, 5.49; found: $\mathrm{C}_{16} \mathrm{H}_{17} \mathrm{NO}_{2}$ : C, 75.42; $\mathrm{H}, 6.93 ; \mathrm{N}, 5.47$.

\section{$N$-[1-(2'-Bromophenyl)ethylidene]aniline, 24.}

It was prepared following the general procedure from 2-bromoacetophenone $(1.35 \mathrm{~mL}, 10$ $\mathrm{mmol})$ and aniline $(0.91 \mathrm{~mL}, 10 \mathrm{mmol})$. The resulting residue was purified by flash chromatography (EtOAC:hexane, 1:5) to give compound $\mathbf{2 4}$ ( $2.44 \mathrm{~g}, 89 \%$ ) as a yellow oil $(E / Z ; 2: 3):{ }^{1} \mathrm{H}$ NMR $\left(300 \mathrm{MHz}, \mathrm{CDCl}_{3}\right) \delta 7.60(\mathrm{dd}, 1 \mathrm{H}, J=0.7$ and $7.9 \mathrm{~Hz}), 7.43(\mathrm{dd}, 2 \mathrm{H}, J=$ 1.5 and 7.5 Hz), 7.39-7.33 (m, 4H), 7.25-7.20 (m, 2H), 7.14-6.99 (m, 5H), 6.90-6.86 (m, 4H), $6.74(\mathrm{dd}, 1 \mathrm{H}, J=1.2$ and $8.4 \mathrm{~Hz}), 2.51(\mathrm{~s}, 2 \mathrm{H}), 2.18(\mathrm{~s}, 3 \mathrm{H}) \mathrm{ppm} ;{ }^{13} \mathrm{C} \mathrm{NMR}\left(75 \mathrm{MHz}, \mathrm{CDCl}_{3}\right)$ $\delta$ (2 isomers) $169.5,168.8,150.5,150.3,143.0,140.6,166.1,132.5,129.9,129.4,129.0$, $128.7,128.5,128.3,127.5,127.0,123.0,123.7,123.4,120.2$, 120.1, 119.2, 28.1, 21.2 ppm.

\section{$N$-[1-(3'-Bromophenyl)ethylidene]-p-methoxyaniline, 25.}

It was prepared following the general procedure from 3-bromoacetophenone $(1.35 \mathrm{~mL}, 10$ mmol) and $p$-methoxyaniline $(1.23 \mathrm{~g}, 10 \mathrm{mmol})$. The resulting residue was purified by crystallization from hexane to give compound 25 (2.40 g, 79\%) as a yellow solid: $\mathrm{mp}$ 73-74 ${ }^{\circ} \mathrm{C} ;{ }^{1} \mathrm{H}$ NMR $\left(300 \mathrm{MHz}, \mathrm{CDCl}_{3}\right) \delta 8.14-8.13(\mathrm{~m}, 1 \mathrm{H}), 7.88-7.84(\mathrm{~m}, 1 \mathrm{H}), 7.59-7.56(\mathrm{~m}, 1 \mathrm{H})$, 7.33-7.26 (m, 1H), 6.94-6.89 (m, 2H), 6.77-7.72 (m, 2H), $3.82(\mathrm{~s}, 3 \mathrm{H}), 2.23(\mathrm{~s}, 3 \mathrm{H}) \mathrm{ppm} ;{ }^{13} \mathrm{C}$ NMR $\left(75 \mathrm{MHz}, \mathrm{CDCl}_{3}\right) \delta 164.4,156.3,144.4,141.9,133.4,130.4,130.0,125.9,122.9$, $120.9,114.25,55.7,17.5 \mathrm{ppm}$. 


\section{$N$-[1-(4'-Bromophenyl)ethylidene]-p-methoxyaniline, 26.}

It was prepared following the general procedure from $p$-bromoacetophenone $(1.35 \mathrm{~mL}, 10$ mmol) and $p$-methoxyaniline $(1.23 \mathrm{~g}, 10 \mathrm{mmol})$. The resulting residue was purified by crystallization from hexane to give compound $26(2.40 \mathrm{~g}, 79 \%)$ as a yellow solid: $\mathrm{mp}$ 112$113{ }^{\circ} \mathrm{C} ;{ }^{1} \mathrm{H}$ NMR $\left(300 \mathrm{MHz}, \mathrm{CDCl}_{3}\right) \delta 7.84(\mathrm{~d}, 2 \mathrm{H}, J=9.0 \mathrm{~Hz}), 7.56(\mathrm{~d}, 2 \mathrm{H}, J=8.5 \mathrm{~Hz}), 6.91$ $(\mathrm{d}, 2 \mathrm{H}, J=9.0 \mathrm{~Hz}), 6.74(\mathrm{~d}, 2 \mathrm{H}, J=8.5 \mathrm{~Hz}), 3.82(\mathrm{~s}, 3 \mathrm{H}), 2.23(\mathrm{~s}, 3 \mathrm{H}) \mathrm{ppm} ;{ }^{13} \mathrm{C} \mathrm{NMR}(75$ $\left.\mathrm{MHz}, \mathrm{CDCl}_{3}\right) \delta 164.5,156.1,144.4,138.6,131.5$ (2C), 128.7 (2C), 124.9, 120.7 (2C), 114.2 (2C), 55.5, 17.1 ppm; EA: calcd for: $\mathrm{C}_{15} \mathrm{H}_{14} \mathrm{BrNO}$ : C, 59.23; H, 4.64; N, 4.60; found: $\mathrm{C}_{15} \mathrm{H}_{14} \mathrm{BrNO}$ : C, 59.11; H, 4.70; N, 4.37.

\section{$N$-[1-(p-Nitrophenyl)ethylidene]-p-methoxyaniline, 27.}

It was prepared following the general procedure from $p$-nitroacetophenone $(1.65 \mathrm{~g}, 10 \mathrm{mmol})$ and $p$-methoxyaniline $(1.23 \mathrm{~g}, 10 \mathrm{mmol})$. The resulting residue was purified by crystallization from hexane to give compound $27(2.43 \mathrm{~g}, 90 \%)$ as a yellow solid: $\mathrm{mp} 94-95{ }^{\circ} \mathrm{C} ;{ }^{1} \mathrm{H}$ NMR $\left(300 \mathrm{MHz}, \mathrm{CDCl}_{3}\right) \delta 8.31(\mathrm{~d}, 2 \mathrm{H}, J=9.0 \mathrm{~Hz}), 8.15(\mathrm{~d}, 2 \mathrm{H}, J=9.0 \mathrm{~Hz}), 6.96(\mathrm{~d}, 2 \mathrm{H}, J=8.8$ $\mathrm{Hz}), 6.80(\mathrm{~d}, 2 \mathrm{H}, J=8.8 \mathrm{~Hz}), 3.85(\mathrm{~s}, 3 \mathrm{H}), 2.34(\mathrm{~s}, 3 \mathrm{H}) \mathrm{ppm} ;{ }^{13} \mathrm{C} \mathrm{NMR}\left(75 \mathrm{MHz}, \mathrm{CDCl}_{3}\right)$ $\delta 163.5,156.7,148.9,145.3,143.8,128.1,123.5,120.8,114.4,55.5,17.4$ ppm.

\section{N-[1-(2'-Naphthyl)ethylidene]aniline, 28.}

It was prepared following the general procedure from 2-acetonaphthone $(1.70 \mathrm{~mL}, 10 \mathrm{mmol})$ and aniline $(0.91 \mathrm{~mL}, 10 \mathrm{mmol})$. The resulting residue was purified by flash chromatography (EtOAC:hexane, 1:5) to give compound 28 (2.38 g, 97\%) as a yellow solid: ${ }^{1} \mathrm{H}$ NMR (300 $\left.\mathrm{MHz}, \mathrm{CDCl}_{3}\right) \delta 8.33(\mathrm{~s}, 1 \mathrm{H}), 8.22(\mathrm{dd}, 1 \mathrm{H}, J=1.8$ y $8.6 \mathrm{~Hz}), 7.94-7.85(\mathrm{~m}, 3 \mathrm{H}), 7.56-7.49(\mathrm{~m}$, 2H), $7.37(\mathrm{td}, 2 \mathrm{H}, J=1.9$ and $7.4 \mathrm{~Hz}), 7.10(\mathrm{td}, 1 \mathrm{H}, J=1.1$ and $7.4 \mathrm{~Hz}), 6.84(\mathrm{dd}, 2 \mathrm{H}, J=1.2$ and $7.5 \mathrm{~Hz}), 2.35$ (s, 3H) ppm; ${ }^{13} \mathrm{C} \mathrm{NMR}\left(75 \mathrm{MHz}, \mathrm{CDCl}_{3}\right) \delta 165.4,151.9,136.9,134.5$, 133.0, 129.1, 129.0, 128.1, 127.8, 127.3, 126.5, 124.3, 123.4, 119.6, 17.5 ppm.

\section{$N$-[1-(2'-Naphthyl)ethylidene]-p-methoxyaniline, 29.}

It was prepared following the general procedure from 2-acetonaphthone $(1.70 \mathrm{~mL}, 10 \mathrm{mmol})$ and $p$-methoxyaniline $(1.23 \mathrm{~g}, 10 \mathrm{mmol})$. The resulting residue was purified by flash chromatography (EtOAC:hexane, 1:10) to give compound $29(2.45 \mathrm{~g}, 89 \%)$ as a yellow solid: mp 148-150 ${ }^{\circ} \mathrm{C} ;{ }^{1} \mathrm{H}$ NMR $\left(300 \mathrm{MHz}, \mathrm{CDCl}_{3}\right) \delta 8.33$ (s, 1H), 8.22 (dd, $1 \mathrm{H}, J=1.6$ and 8.6 $\mathrm{Hz}), 7.94-7.86(\mathrm{~m}, 3 \mathrm{H}), 7.56-7.49(\mathrm{~m}, 2 \mathrm{H}), 6.96-6.91(\mathrm{~m}, 2 \mathrm{H}), 6.83-6.78(\mathrm{~m}, 2 \mathrm{H}), 3.83$ (s, 
3H), 2.38(s, 3H) ppm; ${ }^{13} \mathrm{C} \mathrm{NMR}\left(75 \mathrm{MHz}, \mathrm{CDCl}_{3}\right) \delta 165.7,156.2,145.0,137.3,134.6$, $133.1,129.0,128.2,127,8,127.7,127.3,126.5,124.4,121.0,114.5,55.7,17.5$ ppm.

\section{Hydrosilylation of amines. General method.}

To a solution of corresponding imine (0.2 mmol, 1 equiv.), SulPhos (0.02 mmol, $10 \mathrm{~mol} \%)$ and 2,6-lutidine $(7 \mu \mathrm{L}, 0.06 \mathrm{mmol})$ in toluene $(2 \mathrm{~mL})$, at $-78^{\circ} \mathrm{C}$, under $\operatorname{argon}$ atmosphere,trichlorosilane ( $40 \mu \mathrm{L}, 0.4 \mathrm{mmol}$ ) was added.After stirring for $24 \mathrm{~h}$ at $-78^{\circ} \mathrm{C}$, the reaction mixture was quenched with a saturated $\mathrm{NaHCO}_{3}$ aqueous solution $(2 \mathrm{~mL})$. The aqueous phase was then extracted with $\mathrm{CH}_{2} \mathrm{Cl}_{2}(3 \times 10 \mathrm{~mL})$, the combined organic phases werewashed with a saturated $\mathrm{NaCl}$ aqueous solution $(10 \mathrm{~mL})$ and dried with anhydrous $\mathrm{Na}_{2} \mathrm{SO}_{4}$. The solvent was evaporated under reduced pressure and the reaction crude was purified by flash chromatography $(i \operatorname{Pr} / \mathrm{Hexano}, 1: 100)$ to obtain the desired $N$-arylamine.

\section{(R)- $N$-(1-Phenyletil)aniline, $14 R$.}

It was prepared following the general procedure from $N$-(1-phenylethylidene]aniline 13 (39.05 $\mathrm{mg}, 0.2 \mathrm{mmol}$ ). The resulting residue was purified by flash chromatography to give compound $14 R(35,9 \mathrm{mg}, 91 \%)$ as a colourless oil: $[\alpha]_{\mathrm{D}}{ }^{20}=-4.9\left(c 1.0\right.$ in $\left.\mathrm{CHCl}_{3}\right) ;{ }^{1} \mathrm{H}$ NMR (300 MHz, $\left.\mathrm{CDCl}_{3}\right) \delta 7.38-7.28(\mathrm{~m}, 5 \mathrm{H}), 7.11-7.01(\mathrm{~m}, 2 \mathrm{H}), 6.66-6.60(\mathrm{~m}, 1 \mathrm{H}), 6.52-6.51(\mathrm{~m}$, $2 \mathrm{H}), 4.48(\mathrm{q}, 1 \mathrm{H}, J=6.8 \mathrm{~Hz}), 4.00(\mathrm{bs}, 1 \mathrm{H}), 1.50(\mathrm{~d}, 3 \mathrm{H}, J=6.8 \mathrm{~Hz}) \mathrm{ppm} ;{ }^{13} \mathrm{C} \mathrm{NMR}(100$ $\left.\mathrm{MHz}, \mathrm{CDCl}_{3}\right) \delta 147.7,145.7,129.5,129.1,127.3,126.3,117.6,113.7,53.9,25.5$ ppm; HPLC: 97\% ee, Chiralcel OD-H column (n-hexane/2-propanol, 98/02, 0.5mL/min); $t_{\mathrm{R}}=$ $16.38 \mathrm{~min}$ ( $S$ isomer), $18.99 \mathrm{~min}(R$ isomer).

\section{(R)-N-[(1-Phenyl)ethyl]-p-methoxyaniline, $30 R$.}

It was prepared following the general procedure from $N$-[1-( $p$-phenyl)ethylidene $]-p$ methoxyaniline 20 (45.05 $\mathrm{mg}, 0.2 \mathrm{mmol})$. The resulting residue was purified by flash chromatography to give compound $\mathbf{3 0 R}(43.64 \mathrm{mg}, 96 \%)$ as a white solid: $[\alpha]_{\mathrm{D}}{ }^{20}=+8.6$ $\left(c 0.88\right.$ in $\left.\mathrm{CHCl}_{3}\right)$; ${ }^{1} \mathrm{H}$ NMR $\left(300 \mathrm{MHz}, \mathrm{CDCl}_{3}\right) \delta 7.38-7.28(\mathrm{~m}, 4 \mathrm{H}), 7.25-7.18(\mathrm{~m}, 1 \mathrm{H}), 6.71-$ $6.76(\mathrm{~m}, 2 \mathrm{H}), 6.69-6.44(\mathrm{~d}, 2 \mathrm{H}), 4.41(\mathrm{q}, 1 \mathrm{H}, J=6.8 \mathrm{~Hz}), 3.67(\mathrm{~s}, 3 \mathrm{H}), 1.49$ (d, 3H, J= 6.7 $\mathrm{Hz}) \mathrm{ppm} ;{ }^{13} \mathrm{C} \mathrm{NMR}\left(125 \mathrm{MHz}, \mathrm{CDCl}_{3}\right) \delta 163.7,15.1,145.7,141.8,130.8,128.8,127.0$, 
126.1, 115.0, 114.8, 113.9, 55.9, 54.5, 25.3 ppm; HPLC: 99\% ee, ChiralcelAD column (nhexane/2-propanol, 95/05, $0.5 \mathrm{~mL} / \mathrm{min}) ; t_{\mathrm{R}}=14.37 \mathrm{~min}$ ( $S$ isomer $), 15.61 \mathrm{~min}(R$ isomer $)$.

(R)-N-[1-(p-Methoxyphenyl)ethyl)]aniline, $31 R$.

It was prepared following the general procedure from $N$-[1- $(p-$ methoxyphenyl)ethylidene]aniline 21 (45.05 $\mathrm{mg}, 0.2 \mathrm{mmol})$. The resulting residue was purified by flash chromatography to give compound $\mathbf{3 1} \boldsymbol{R}(43.19 \mathrm{mg}, 95 \%)$ as a white solid: $[\alpha]_{\mathrm{D}}{ }^{20}=-6.2\left(c 0.55\right.$ in $\left.\mathrm{CH}_{3} \mathrm{OH}\right) ;{ }^{1} \mathrm{H}$ NMR $\left(300 \mathrm{MHz}, \mathrm{CDCl}_{3}\right) \delta 7.27(\mathrm{~d}, 2 \mathrm{H}, J=8.0 \mathrm{~Hz})$, 7.11-7.06 (m, 1H), $6.85(\mathrm{~d}, 2 \mathrm{H}, J=8.0 \mathrm{~Hz}), 6.63(\mathrm{t}, 1 \mathrm{H}, J=8.0 \mathrm{~Hz}), 6.51(\mathrm{~d}, 2 \mathrm{H}, J=8.0 \mathrm{~Hz})$, $4.44(\mathrm{q}, 1 \mathrm{H}, J=6.6 \mathrm{~Hz}), 3.77(\mathrm{~s}, 3 \mathrm{H}), 1.48(\mathrm{~d}, 3 \mathrm{H}, J=6.6 \mathrm{~Hz}) \mathrm{ppm} ;{ }^{13} \mathrm{C} \mathrm{NMR}(100 \mathrm{MHz}$, $\left.\mathrm{CDCl}_{3}\right) \delta 158.6,147.5,137.4,129.2,127.0,117.3,114.2,113.5,55.4,53.0,25.2 \mathrm{ppm}$; HRMS: calcd for $\mathrm{C}_{15} \mathrm{H}_{18} \mathrm{NO}$ [M+H]: 228.1388; found: 228.1380; HPLC: 99\% ee, Chiralcel OD-H column (n-hexane/2-propanol, 98/02, $0.5 \mathrm{~mL} / \mathrm{min}$ ); $t_{\mathrm{R}}=23.45 \mathrm{~min}$ ( $S$ isomer), 24.37 $\min (R$ isomer).

(R)-N-[(1-(p-Methoxyphenyl)ethyl]-p-methoxyaniline, $32 R$.

It was prepared following the general procedure from $N$-[1-( $p$-methoxyphenyl)ethylidene]- $p$ methoxyaniline 22 (51.06 $\mathrm{mg}, 0.2 \mathrm{mmol}$ ). The resulting residue was purified by flash chromatography to give compound $\mathbf{3 2 R}(47.90 \mathrm{mg}, 93 \%)$ as a yellow solid: $[\alpha]_{\mathrm{D}}{ }^{20}=+24.2$ $\left(c 1.06\right.$ in $\left.\mathrm{CHCl}_{3}\right) ;{ }^{1} \mathrm{H} \mathrm{NMR}\left(300 \mathrm{MHz}, \mathrm{CDCl}_{3}\right) \delta 7.26(\mathrm{~d}, 2 \mathrm{H}, J=7.8 \mathrm{~Hz}), 6.84(\mathrm{~d}, 2 \mathrm{H}, J=7.8$ Hz), 6.68 (d, 2H, $J=6.6 \mathrm{~Hz}), 6.47$ (d, 2H, $J=6.6 \mathrm{~Hz}), 4.36$ (q, 1H, $J=6.6 \mathrm{~Hz}), 3.77$ (bs, 1H), $3.69(\mathrm{~s}, 3 \mathrm{H}), 1.47$ (d, 3H, $J=6.7 \mathrm{~Hz}) \mathrm{ppm} ;{ }^{13} \mathrm{C} \mathrm{NMR}\left(75 \mathrm{MHz}, \mathrm{CDCl}_{3}\right) \delta 158.4,151.9,141.5$, 137.4, 126.9, 114, 114.6, 113.9, 55.7, 54.3, 53.6, 25.0 ppm; HPLC: $\geq 99 \%$ ee, Chiralcel OD-H column (n-hexane/2-propanol, 95/05, $0.5 \mathrm{~mL} / \mathrm{min}) ; t_{\mathrm{R}}=20.53 \mathrm{~min}$ ( $R$ isomer), $23.58 \mathrm{~min}(S$ isomer).

\section{(R)-N-[(1-(3'-Methoxyphenyl)ethyl]-p-methoxyaniline, 33R.}

It was prepared following the general procedure from $N$-[1-(3'-methoxyphenyl)ethylidene $]-p$ methoxyaniline 23 (51.06 $\mathrm{mg}, 0.2 \mathrm{mmol}$ ). The resulting residue was purified by flash chromatography to give compound $\mathbf{3 3 R}(48.36 \mathrm{mg}, 94 \%)$ as a yellow solid: ${ }^{1} \mathrm{H}$ NMR (300 $\left.\mathrm{MHz}, \mathrm{CDCl}_{3}\right) \delta 7.30(\mathrm{t}, 1 \mathrm{H}, J=7.8 \mathrm{~Hz}), 7.04-7.00(\mathrm{~m}, 2 \mathrm{H}), 6.83(\mathrm{ddd}, 1 \mathrm{H}, J=8.3,2.8$ and 0.8 $\mathrm{Hz}), 6.76(\mathrm{~d}, 1 \mathrm{H}, J=9.0 \mathrm{~Hz}), 6.55$ (d, 2H, J=9.1 Hz), 4.44 (q, 1H, $J=6.90 \mathrm{~Hz}), 3.84(\mathrm{br}, 1 \mathrm{H})$, 3.83(s, 3H), $3.75(\mathrm{~s}, 3 \mathrm{H}), 1.54(\mathrm{~d}, 3 \mathrm{H}, J=6.9 \mathrm{~Hz}) \mathrm{ppm} ;{ }^{13} \mathrm{C} \mathrm{NMR}\left(75 \mathrm{MHz}, \mathrm{CDCl}_{3}\right) \delta 159.7$, 
151.7, 141.3, 141.4, 129.4, 118.1, 114.6, 111.8, 111.5, 55.6, 55.1, 54.2, 25.1 ppm; HPLC: 97\% ee, Chiralcel OD-H column (n-hexane/2-propanol, 96/04, $0.5 \mathrm{~mL} / \mathrm{min}$ ); $t_{\mathrm{R}}=24.31 \mathrm{~min}$ ( $R$ isomer), $28.42 \min (S$ isomer).

(R)-N-[(1-(2'-Bromophenyl)ethyl]aniline, 34R.

It was prepared following the general procedure from $N$-[1-(2'bromophenyl)ethylidene]aniline $24(54.83 \mathrm{mg}, 0.2 \mathrm{mmol})$. The resulting residue was purified by flash chromatography to give compound $\mathbf{3 4 R}(51.37 \mathrm{mg}, 93 \%)$ as a yellow solid: ${ }^{1} \mathrm{H}$ NMR $\left(300 \mathrm{MHz}, \mathrm{CDCl}_{3}\right) \delta 7.5(\mathrm{dd}, 1 \mathrm{H}, J=1.2$ and $7.9 \mathrm{~Hz}), 7.44(\mathrm{dd}, 1 \mathrm{H}, J=1.8$ and $7.7 \mathrm{~Hz}$ ), 7.25$7.20(\mathrm{~m}, 1 \mathrm{H}), 7.11-7.05(\mathrm{~m}, 3 \mathrm{H}), 6.67-6.61(\mathrm{~m}, 1 \mathrm{H}), 6.45-6.40(\mathrm{~m}, 2 \mathrm{H}), 4.83(\mathrm{q}, 1 \mathrm{H}, J=7.1$ $\mathrm{Hz}$ ), 4.13 (bs, 1H), 1.49 (d, 3H, $J=8 \mathrm{~Hz}) \mathrm{ppm} ;{ }^{13} \mathrm{C} \mathrm{NMR}\left(75 \mathrm{MHz}, \mathrm{CDCl}_{3}\right) \delta 146.7,143.5$, 133.0, 129.1, 128.4, 128.0, 126.9, 126.9, 122.6, 117.4, 113.1, 52.7, 23.1 ppm; HPLC: 55\% ee, ChiralcelAD column (n-hexane/2-propanol, 95/05, 0.6mL/min); $t_{\mathrm{R}}=8.82 \mathrm{~min}(S$ isomer), $9.71 \mathrm{~min}(\mathrm{R}$ isomer).

(R)-N-[(1-(3'-Bromophenyl)ethyl]-p-methoxyaniline, $35 R$.

It was prepared following the general procedure from $N-\left[1-\left(3^{\prime}\right.\right.$-bromophenyl)ethylidene $]-p$ methoxyaniline 25 (55.12 $\mathrm{mg}, 0.2 \mathrm{mmol})$. The resulting residue was purified by flash chromatography to give compound $\mathbf{3 5 R}(51.37 \mathrm{mg}, 90 \%)$ as a yellow solid: ${ }^{1} \mathrm{H}$ NMR (300 $\left.\mathrm{MHz}, \mathrm{CDCl}_{3}\right) \delta 8.13(\mathrm{t}, 1 \mathrm{H}, J=1.8 \mathrm{~Hz}), 7.86(\mathrm{~d}, 1 \mathrm{H}, J=8.0 \mathrm{~Hz}), 7.57(\mathrm{dq}, 1 \mathrm{H}, J=0.9$ and $7.9 \mathrm{~Hz}), 7.30(\mathrm{t}, 1 \mathrm{H}, J=8.0 \mathrm{~Hz}), 6.91(\mathrm{~d}, 2 \mathrm{H}, J=6.8 \mathrm{~Hz}), 6.74(\mathrm{~d}, 2 \mathrm{H}, J=6.8 \mathrm{~Hz}), 4.35(\mathrm{q}, 1 \mathrm{H}$, $J=6.8 \mathrm{~Hz}), 3.69(\mathrm{~s}, 3 \mathrm{H}), 1.46(\mathrm{~d}, 3 \mathrm{H}, J=6.8 \mathrm{~Hz}) \mathrm{ppm} ;{ }^{13} \mathrm{C} \mathrm{NMR}\left(75 \mathrm{MHz}, \mathrm{CDCl}_{3}\right) \delta 152.5$, 148.6, 141.6, 130.7 130.4, 129.5, 125.0, 123.2, 115.2, 115.0, 56.2, 54.40, 24.6 ppm; HPLC: $\geq 99 \%$ ee, ChiralcelAD column (n-hexane/2-propanol, 98/02, $0.6 \mathrm{~mL} / \mathrm{min}) ; t_{\mathrm{R}}=8.90 \mathrm{~min}(R$ isomer), $10.99 \mathrm{~min}$ ( $S$ isomer).

$(R)-N$-[(1-(p-Bromophenyl)ethyl]-p-methoxyaniline, $36 R$.

It was prepared following the general procedure from $N$-[1-( $p$-bromophenyl)ethylidene $]-p$ methoxyaniline 26 (60.60 $\mathrm{mg}, 0.2 \mathrm{mmol})$. The resulting residue was purified by flash chromatography to give compound $\mathbf{3 6} \boldsymbol{R}(56.34 \mathrm{mg}, 92 \%)$ as a yellow solid: ${ }^{1} \mathrm{H}$ NMR (400 $\left.\mathrm{MHz}, \mathrm{CDCl}_{3}\right) \delta 7.42(\mathrm{~d}, 2 \mathrm{H}, J=8.3 \mathrm{~Hz}), 7.23(\mathrm{~d}, 2 \mathrm{H}, J=8.3 \mathrm{~Hz}), 6.68(\mathrm{~d}, 2 \mathrm{H}, J=9.0 \mathrm{~Hz})$, $6.42(\mathrm{~d}, 2 \mathrm{H}, J=9.0 \mathrm{~Hz}), 4.35$ (q, 1H, $J=6.8 \mathrm{~Hz}), 3.69$ (s, 3H), 1.56 (bs, 1H), 1.47 (d, 3H, $J=$ $6.8 \mathrm{~Hz}) \mathrm{ppm} ;{ }^{13} \mathrm{C} \mathrm{NMR}\left(100 \mathrm{MHz}, \mathrm{CDCl}_{3}\right) \delta 152.3,144.8,141.4,131.9,127.9,120.6,115.0$, 
114.7, 55.9, 54.0, 25.3 ppm; HPLC: 97\% ee, ChiralcelOD-H column (n-hexane/2-propanol, $98 / 02,0.6 \mathrm{~mL} / \mathrm{min}) ; t_{\mathrm{R}}=27.00 \mathrm{~min}(R$ isomer $), 33.17 \mathrm{~min}(S$ isomer $)$.

\section{$(R)-N-[(1-(p-N i t r o p h e n y l) e t h y l]-p$-methoxyaniline, $37 R$.}

It was prepared following the general procedure from $N$-[1-(p-nitrophenyl)ethylidene $]-p$ methoxyaniline $27(52.83 \mathrm{mg}, 0.2 \mathrm{mmol})$. The resulting residue was purified by flash chromatography to give compound $\mathbf{3 7 R}(56.34 \mathrm{mg}, 97 \%)$ as a yellow solid: $[\alpha]_{\mathrm{D}}{ }^{20}=+22.6$ $\left(c 0.92\right.$ in $\left.\mathrm{CHCl}_{3}\right) ;{ }^{1} \mathrm{H} \mathrm{NMR}\left(300 \mathrm{MHz}, \mathrm{CDCl}_{3}\right) \delta 8.18(\mathrm{~d}, J=8.8 \mathrm{~Hz}, 2 \mathrm{H}), 7.54$ (d, J=8.5 Hz, 2H), 6.71-6.66 (m, 2H), 6.42-6.37 (m, 2H), 4.50 (q, 1H, J=6.6 Hz), 3.69 (s, 3H), 1.52 (d, 3H, $J=6.6 \mathrm{~Hz}) \mathrm{ppm} ;{ }^{13} \mathrm{C} \mathrm{NMR}\left(100 \mathrm{MHz}, \mathrm{CDCl}_{3}\right) \delta 152.3,147.0,140.8,126.8,124.0,114.7$, 114.6, 55.8, 54.0, 25.0 ppm; HPLC: 99\% ee, Chiralcel AD column (n-hexane/2-propanol, $98 / 02,1.0 \mathrm{~mL} / \mathrm{min}) ; t_{\mathrm{R}}=37.68 \mathrm{~min}$ ( $S$ isomer $), 40.66 \mathrm{~min}$ ( $R$ isomer $)$.

\section{(R)-N-[(1-(2'-Naphthyl)ethyl]aniline, 38R.}

It was prepared following the general procedure from $N$-[1-(2'-naphthyl)ethylidene $]$ aniline 28 (49.06 $\mathrm{mg}, 0.2 \mathrm{mmol}$ ). The resulting residue was purified by flash chromatography to give compound $38 R(47.98 \mathrm{mg}, 97 \%)$ as a yellow solid: $[\alpha]_{\mathrm{D}}{ }^{20}=-17.5\left(c 0.61\right.$ in $\left.\mathrm{CHCl}_{3}\right) ;{ }^{1} \mathrm{H} \mathrm{NMR}$ $\left(500 \mathrm{MHz}, \mathrm{CDCl}_{3}\right) \delta 7.87-7.83(\mathrm{~m}, 4 \mathrm{H}), 7.56-7.54(\mathrm{~m}, 1 \mathrm{H}), 7.52-7.46(\mathrm{~m}, 2 \mathrm{H}), 7.15-7.11(\mathrm{~m}$, 2H), 6.70-6.68 (m, 1H), 6.61-6.59 (m, 2H), 4.69 (q, J=6.8 Hz, 1H), $1.64(\mathrm{~d}, J=6.8 \mathrm{~Hz}, 3 \mathrm{H})$ ppm; ${ }^{13} \mathrm{C}$ NMR $\left(125 \mathrm{MHz}, \mathrm{CDCl}_{3}\right) \delta 147.5,142.9,133.8,132.9,129.3,128.7,128.0,127.8$, 126.2, 125.7, 124.6, 124.4, 117.5, 113.6, 53.9, 25.2 ppm; HPLC: 99\% ee, Chiralcel AD column (n-hexane/2-propanol, 98/02, $0.6 \mathrm{~mL} / \mathrm{min}) ; t_{\mathrm{R}}=13.86 \mathrm{~min}$ ( $S$ isomer $), 16.30 \mathrm{~min}(R$ isomer).

(R)-N-[1-(2'-Naphthyl)ethyl]-p-methoxyaniline, 39R.

It was prepared following the general procedure from $N$-[1-(2'-naphthyl)ethylidene $]$ aniline 29 (55.06 $\mathrm{mg}, 0.2 \mathrm{mmol})$. The resulting residue was purified by flash chromatography to give compound 39R $(53,81 \mathrm{mg}, 97 \%)$ as a white solid: $[\alpha]_{\mathrm{D}}{ }^{20}=+11.1\left(c 1.30\right.$ in $\left.\mathrm{CHCl}_{3}\right)$; ${ }^{1} \mathrm{H}$ NMR $\left(500 \mathrm{MHz}, \mathrm{CDCl}_{3}\right) \delta 7.82-7.77(\mathrm{~m}, 4 \mathrm{H}), 7.51-7.41(\mathrm{~m}, 2 \mathrm{H}), 6.68-6.62(\mathrm{~m}, 2 \mathrm{H}), 6.53-6.48(\mathrm{~m}$, $2 \mathrm{H}), 4.56(\mathrm{q}, J=6.8 \mathrm{~Hz}, 1 \mathrm{H}), 3.67(\mathrm{~s}, 3 \mathrm{H}), 1.56(\mathrm{~d}, 3 \mathrm{H}, J=6.8 \mathrm{~Hz}) \mathrm{ppm} ;{ }^{13} \mathrm{C} \mathrm{NMR}(100$ $\left.\mathrm{MHz}, \mathrm{CDCl}_{3}\right) \delta 152.0,142.9,141.4,133.6,132.7,128.4,127.8,127.6,159.9,125.5,124.4$, 124.3, 114.8, 55.7, 54.6, 25.1 ppm; HPLC: $\geq 99 \%$ ee, ChiralcelOD-H column (n-hexane/2propanol, 95/05, 0.5mL/min); $t_{\mathrm{R}}=17.96 \mathrm{~min}$ ( $R$ isomer), $21.06 \mathrm{~min}$ ( $S$ isomer). 
(R)-1-(3-Methylphenyl) ethylamine, 40R.

A solution of $\mathrm{N}$-(4-Methoxyphenyl)-1-(3-methoxyphenyl) ethylamina $33 \boldsymbol{R}$ (74 $\mathrm{mg}, 0.49$ mmol) in $\mathrm{MeOH} / \mathrm{H}_{2} \mathrm{O}$ (4:1) was stirred during 10 minutes, cooled to $0^{\circ} \mathrm{C}$, and then $\mathrm{CAN}$ $(1.06 \mathrm{~g}, 1.94 \mathrm{mmol})$ was added in a portion. The reaction mixture was stirred during $6 \mathrm{~h}$ at $0^{\circ} \mathrm{C}$ and then, $\mathrm{MeOH}$ was evaporated under reduced pressure. The residue obtained was solved in $\mathrm{CH}_{2} \mathrm{Cl}_{2}(15 \mathrm{~mL})$ and a solution of $1 \mathrm{~N} \mathrm{HCl}$ was added until $\mathrm{pH}=2$ was reached. The reaction mixture was washed with $\mathrm{CH}_{2} \mathrm{Cl}_{2}(2 \times 15 \mathrm{~mL})$ and a solution of $1 \mathrm{~N} \mathrm{NaOH}$ was added to the aqueous layer until alkaline $\mathrm{pH}$. The aqueous phase was then extracted with $\mathrm{CH}_{2} \mathrm{Cl}_{2}(2 \times 15$ $\mathrm{mL}$ ), the combined organic phases were washed with a saturated $\mathrm{NaCl}$ aqueous solution (15 $\mathrm{mL}$ ) and dried with anhydrous $\mathrm{Na}_{2} \mathrm{SO}_{4}$. The solvent was evaporated under reduced pressure and the reaction crude was purified by flash chromatography (ether/hexane, 1:8) to obtain the desired compound $\mathbf{4 0 R}(61.5 \mathrm{mg}, 83 \%)$ as a colorless oil: $[\alpha]_{\mathrm{D}}{ }^{20}=+17.6(c 0.2$ in $\mathrm{MeOH}) ;{ }^{1} \mathrm{H}$ NMR (500 MHz, $\left.\mathrm{CDCl}_{3}\right)$ 87.19-7.16 (m, 1H), 6.86-6.84 (m, 1H), 6.72-6.69 (m, 1H), 4.03 (q, $J=6.6 \mathrm{~Hz}, 1 \mathrm{H}), 3.74(\mathrm{~s}, 3 \mathrm{H}), 2.01(\mathrm{bs}, 2 \mathrm{H}), 1.33$ (d, 3H, $J=6.6 \mathrm{~Hz}) \mathrm{ppm} ;{ }^{13} \mathrm{C} \mathrm{NMR}(125$ $\left.\mathrm{MHz}, \mathrm{CDCl}_{3}\right) \delta 159.9,149.0,129.5,118.1,112.3,111.4,55.2,51.4,25.3$ ppm;EA: calcd for: $\mathrm{C}_{9} \mathrm{H}_{13} \mathrm{NO}$ : C, 71.49; H, 8.67; N, 9.26; found: $\mathrm{C}_{9} \mathrm{H}_{13} \mathrm{NO}$ : C, 67.86; H, 8.42; N, 8.77; HRMS: $\mathrm{C}_{9} \mathrm{H}_{14} \mathrm{NO}[\mathrm{M}+\mathrm{H}]:$ 152.1075; found: 152.1069 .

\section{(R)- $N$-[1-(3-Methoxyphenyl)ethyl] 3-(2-chlorophenyl)propanamide, $42 R$.}

To a solution of 3-(2-chlorophenyl) propionic acid 41 (48 $\mathrm{mg}, 0.257 \mathrm{mmol}$ ) at room temperature, under argon atmosphere, in dry $\mathrm{CH}_{2} \mathrm{Cl}_{2}(2 \mathrm{~mL}$ ), dicyclocarbodiimide (DCC) (58 $\mathrm{mg}, 0.282 \mathrm{mmol}$ ) anddimethylaminopyridine (DMAP) (3.16 mg, 0.10 equiv.) were added. After stirring for $1 \mathrm{~h}$ at $\mathrm{rt}, \mathbf{4 0 R}(38.8 \mathrm{mg}, 0.257 \mathrm{mmol})$ was added and then stirring during $1 \mathrm{~h}$. The reaction mixture was filtered through a pad of Celite and the solvent was evaporated under reduced pressure.The obtained crude was purified by flash chromatography (EtOAc:hexane, 3:1) to obtain the desired compound $\mathbf{4 2 R}(78,41 \mathrm{mg}, 96 \%$ ) as a white solid:mp $96^{\circ} \mathrm{C} ;[\alpha]_{\mathrm{D}}{ }^{20}=+47\left(c 1.0\right.$ in $\left.\mathrm{CHCl}_{3}\right) ;{ }^{1} \mathrm{H}$ NMR $\left(500 \mathrm{MHz}, \mathrm{CDCl}_{3}\right) \delta 7.31-7.20(\mathrm{~m}$, $5 \mathrm{H}), 6.82-6.80(\mathrm{~m}, 3 \mathrm{H}), 5.51(\mathrm{~d}, 1 \mathrm{H}, J=7.0 \mathrm{~Hz}), 5.09$ (c, $1 \mathrm{H}, J=7.0 \mathrm{~Hz}), 3.81(\mathrm{~s}, 3 \mathrm{H}), 2.99$ (t, $2 \mathrm{H}, J=7.5 \mathrm{~Hz}), 2.50(\mathrm{t}, 2 \mathrm{H}, J=7.5 \mathrm{~Hz}), 1.42(\mathrm{~d}, 3 \mathrm{H}, J=6.9 \mathrm{~Hz}) \mathrm{ppm} ;{ }^{13} \mathrm{C} \mathrm{NMR}(125 \mathrm{MHz}$, $\left.\mathrm{CDCl}_{3}\right) \delta 171.0,159.8,144.7,140.8,129.7,128.5,128.4,126.2,118.4,112.5,112.3,55.2$, 48.7, 38.6, 31.7, 21.6 ppm; HRMS: calcd for $\mathrm{C}_{18} \mathrm{H}_{21} \mathrm{NO}_{2}[\mathrm{M}+\mathrm{H}-\mathrm{Cl}]^{+}$: 283.1572; found: 283.1570 . 


\section{(R)-N-[1-(3-Methoxyphenyl)ethyl]-3-(2-chlorophenyl)-1-propylamine, NPS R-568.}

To a solution of amide $\mathbf{4 2 R}(80 \mathrm{mg}, 0.25 \mathrm{mmol})$ in ether $(2 \mathrm{~mL})$ at room temperature and

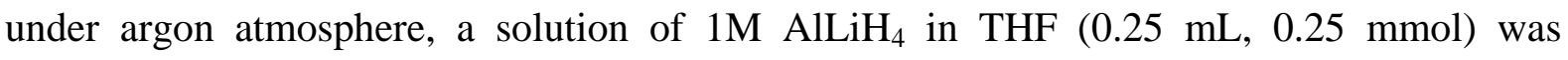
added.After stirring overnight, the reaction mixture was hydrolyzed with a saturated $\mathrm{Na}_{2} \mathrm{SO}_{4}$ aqueous solution $\left(0.3 \mathrm{~mL} / \mathrm{mmolof} \mathrm{AlLiH}_{4}\right)$. The resulting suspension was filtered through a pad of Celite and the solvent was evaporated under reduced pressure. The crude was purified on a cation-exchange column (Isolute SPE SCX-2) using $\mathrm{NH}_{3} / \mathrm{MeOH}(2 \mathrm{M}$ ) as eluent to obtain compound NPS R-568 $(6.33 \mathrm{mg}, 86 \%)$ as a yellow oil: $[\alpha]_{\mathrm{D}}{ }^{20}=+39.4\left(c 1.0\right.$ in $\left.\mathrm{CHCl}_{3}\right)$ [lit. ${ }^{1}[\alpha]_{\mathrm{D}}{ }^{20}=+39.1\left(c 1.0\right.$ in $\left.\mathrm{CHCl}_{3}\right] ;{ }^{1} \mathrm{H}$ NMR $\left(500 \mathrm{MHz}, \mathrm{CDCl}_{3}\right) \delta 7.27-7.22(\mathrm{~m}, 2 \mathrm{H}), 7.18-$ 7.13, (m, 3H), 6.90-6.89 (m, 2H), $6.79(\mathrm{ddd}, 1 \mathrm{H}, J=8.3,2.4$ and $0.9 \mathrm{~Hz}), 3.82(\mathrm{~s}, 3 \mathrm{H}), 3.74$ $(\mathrm{c}, 1 \mathrm{H}, J=6.5 \mathrm{~Hz}), 2.68-2.48(\mathrm{~m}, 4 \mathrm{H}), 1.84-1.78(\mathrm{~m}, 2 \mathrm{H}), 1.36(\mathrm{~d}, 3 \mathrm{H}, J=6.6 \mathrm{~Hz}) \mathrm{ppm} ;{ }^{13} \mathrm{C}$ $\operatorname{NMR}\left(125 \mathrm{MHz}, \mathrm{CDCl}_{3}\right) \delta 159.8,142.1,129.4,128.3,128.2,125.7,119.0,112.3,112.1$, 58.4, 55.2, 47.3, 33.6, 31.7, 24.1 ppm; HRMS: calcd for $\mathrm{C}_{18} \mathrm{H}_{22} \mathrm{NO}[\mathrm{M}-\mathrm{Cl}]^{+}:$268.1701; found: 268.1697 .

\footnotetext{
${ }^{1}$ Fernández, I.; Valdivia, V.; Khiar, N. J Org .Chem. 2008, 73, 745-748.
} 
${ }^{1} \mathrm{H},{ }^{13} \mathrm{C}$ and ${ }^{31} \mathrm{P}-\mathrm{NMR}$ spectra of selected compounds.

$(R)$-(E)-N-[3,5-bis(trifluoromethyl)benzylidene]-2-methyl-2-propanesulfinamide, $3 R$.

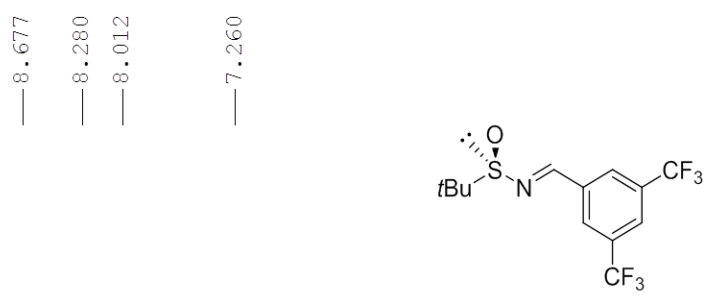

${ }^{1} \mathrm{H}$ NMR $\left(300 \mathrm{MHz}, \mathrm{CDCl}_{3}\right)$

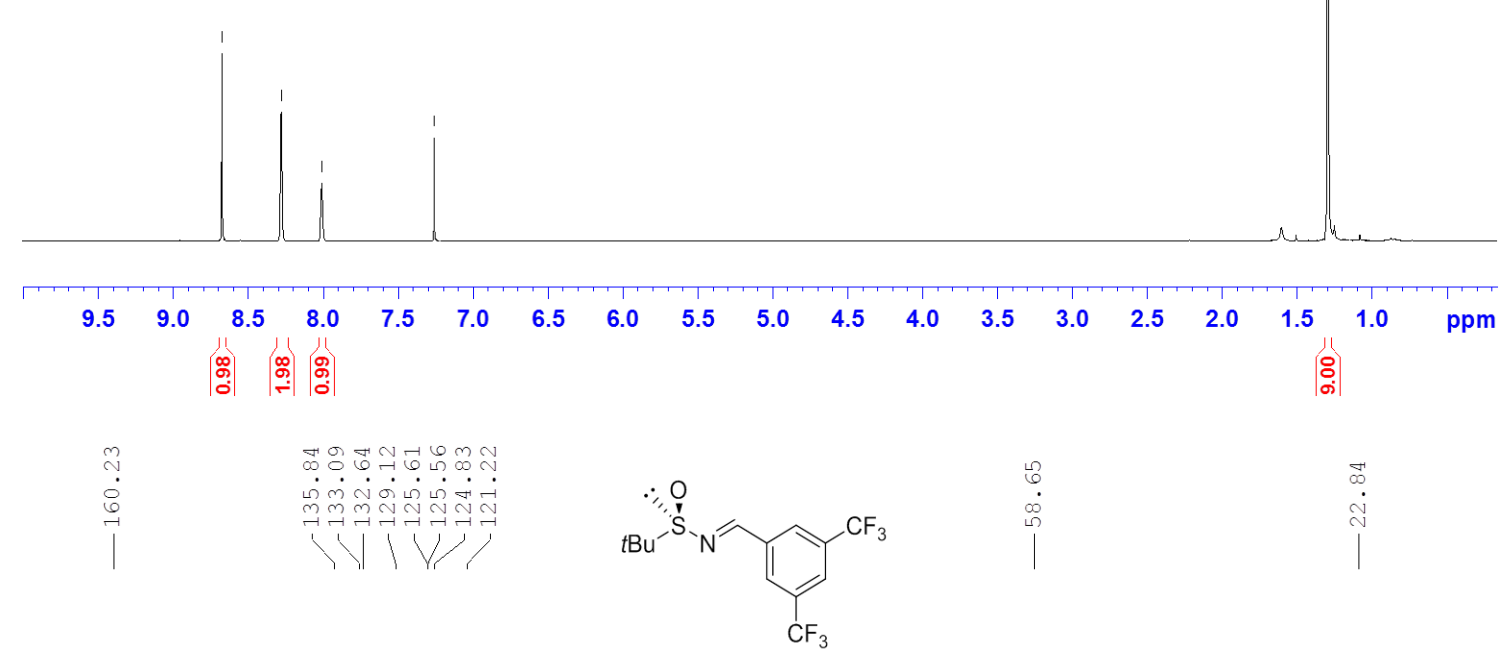

${ }^{13} \mathrm{C} \mathrm{NMR}\left(75 \mathrm{MHz}, \mathrm{CDCl}_{3}\right)$

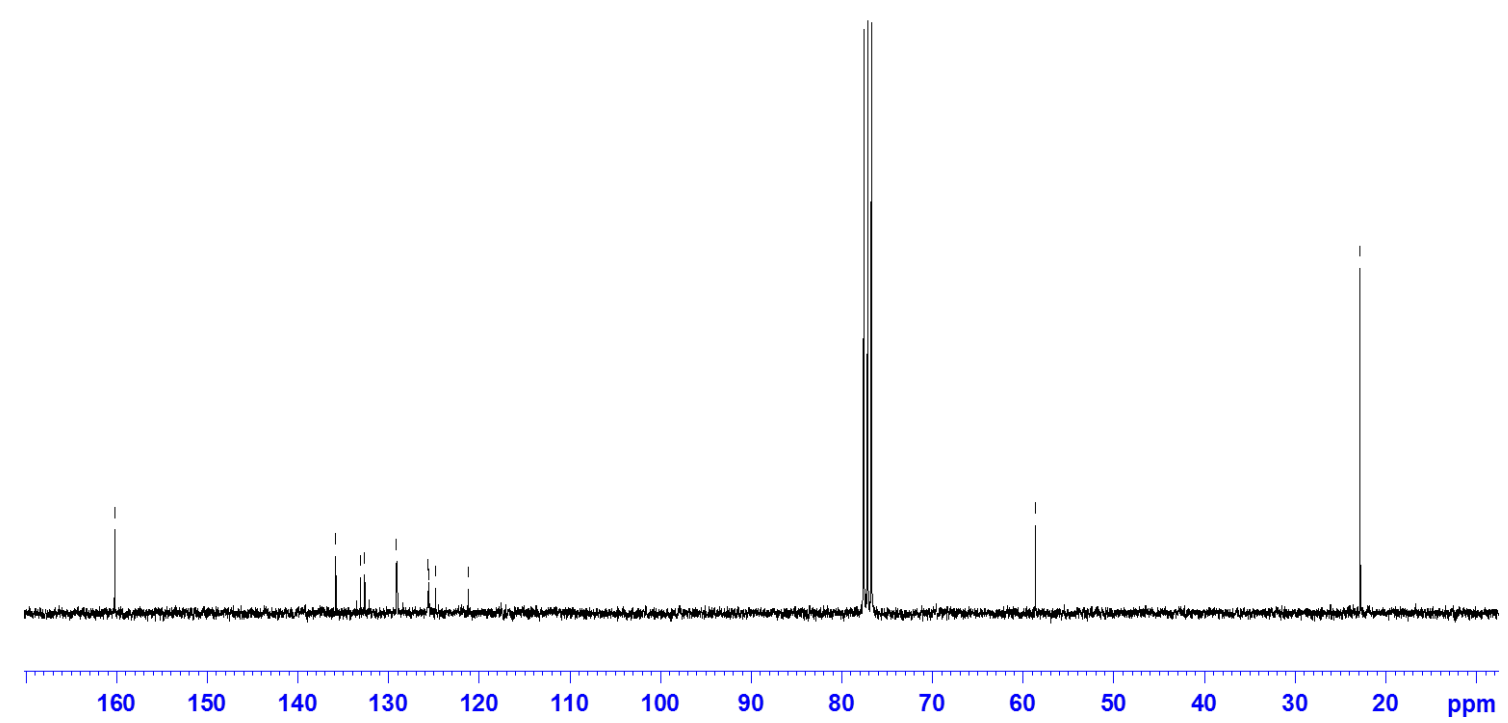


(R)-N-[(2'-naphthyl)methylidene]-2-methyl-2-propanesulfinamide, $4 R$.
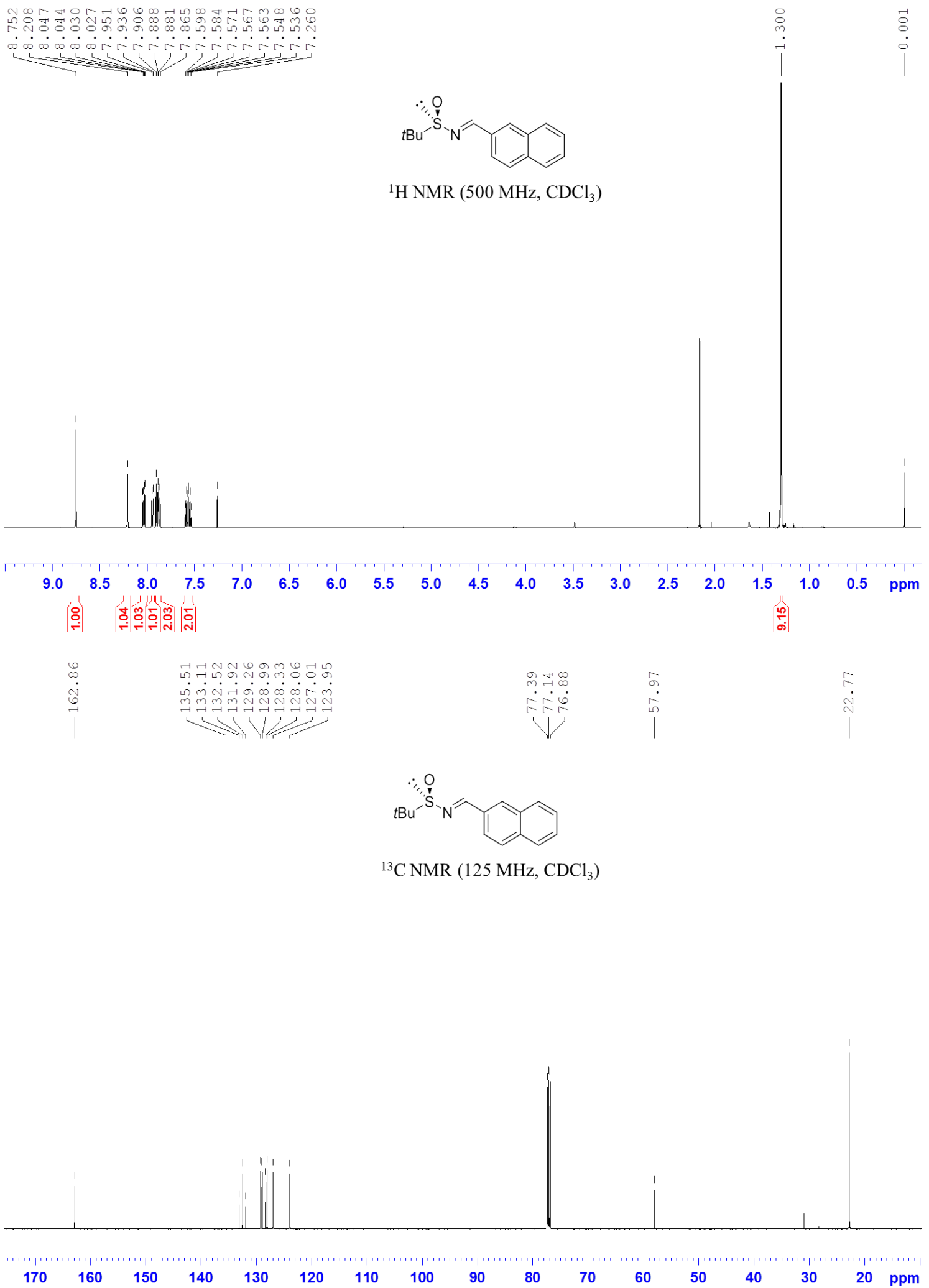
(R)-(E)-N-(ferrocenylmethylidene)-2-methyl-2-propanesulfinamide, $5 R$.
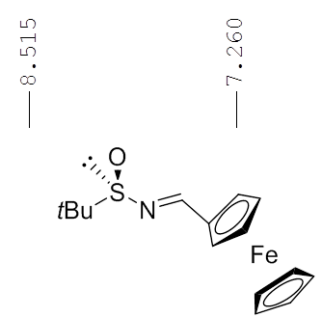

${ }^{1} \mathrm{H}$ NMR $\left(500 \mathrm{MHz}, \mathrm{CDCl}_{3}\right)$
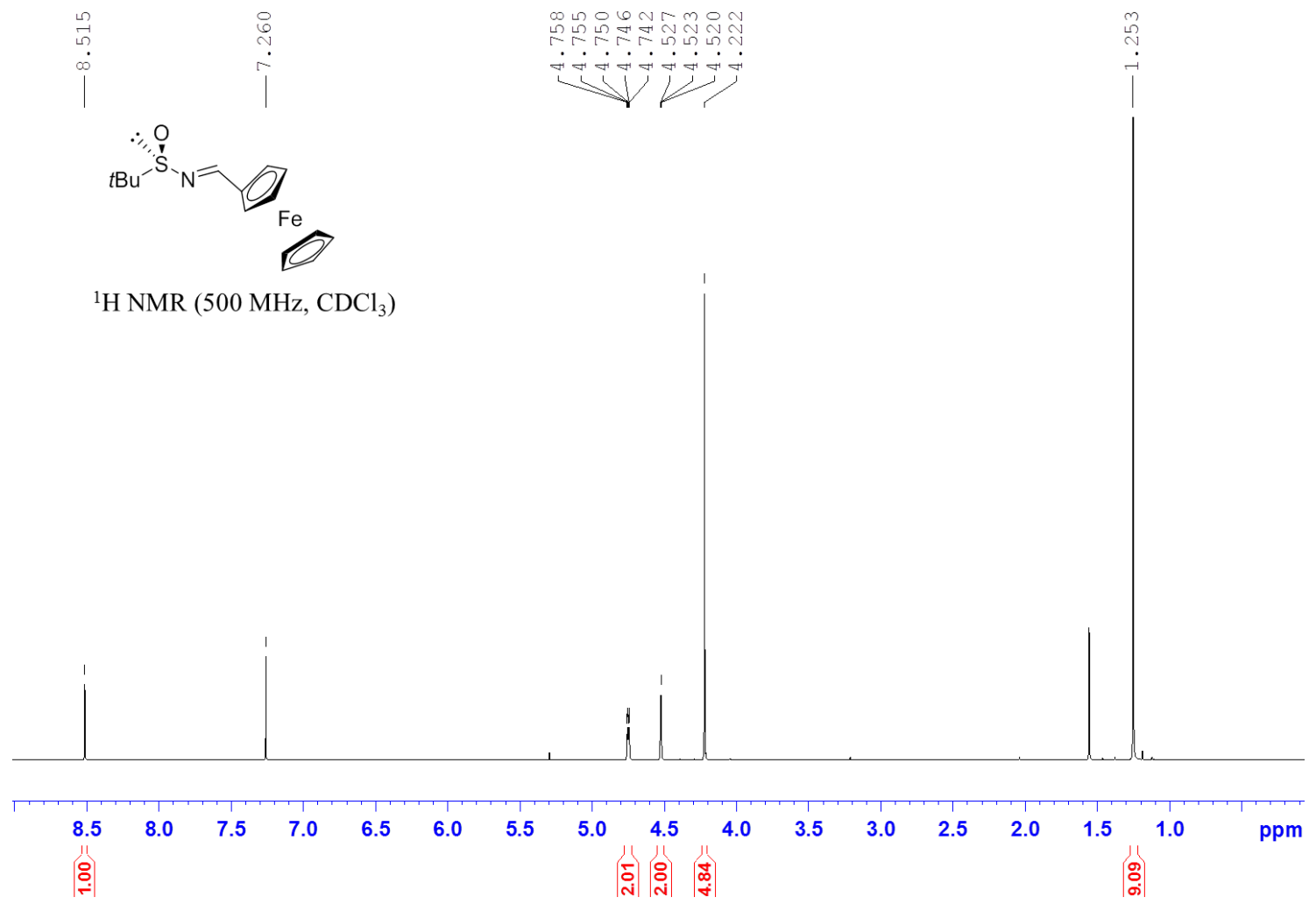

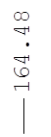

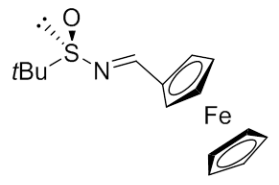

${ }^{13} \mathrm{C}$ NMR (125 MHz, $\mathrm{CDCl}_{3}$ )

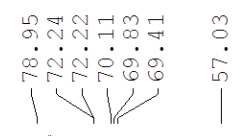

$\vec{\sigma}$

23 
(R)-E-N-(2-methylpropylidene)-2-methyl-2-propanesulfinamide, $6 R$.
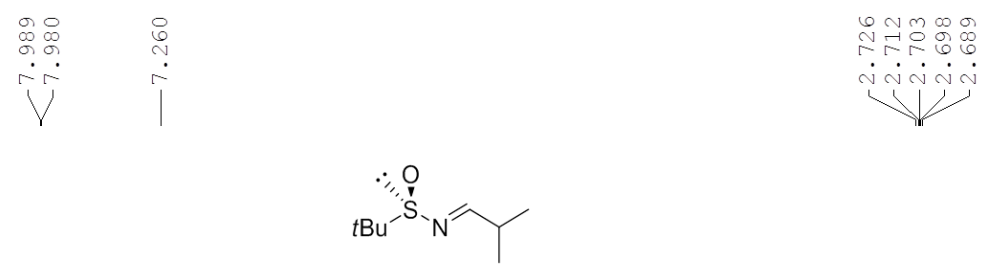

${ }^{1} \mathrm{H}$ NMR $\left(500 \mathrm{MHz}, \mathrm{CDCl}_{3}\right)$
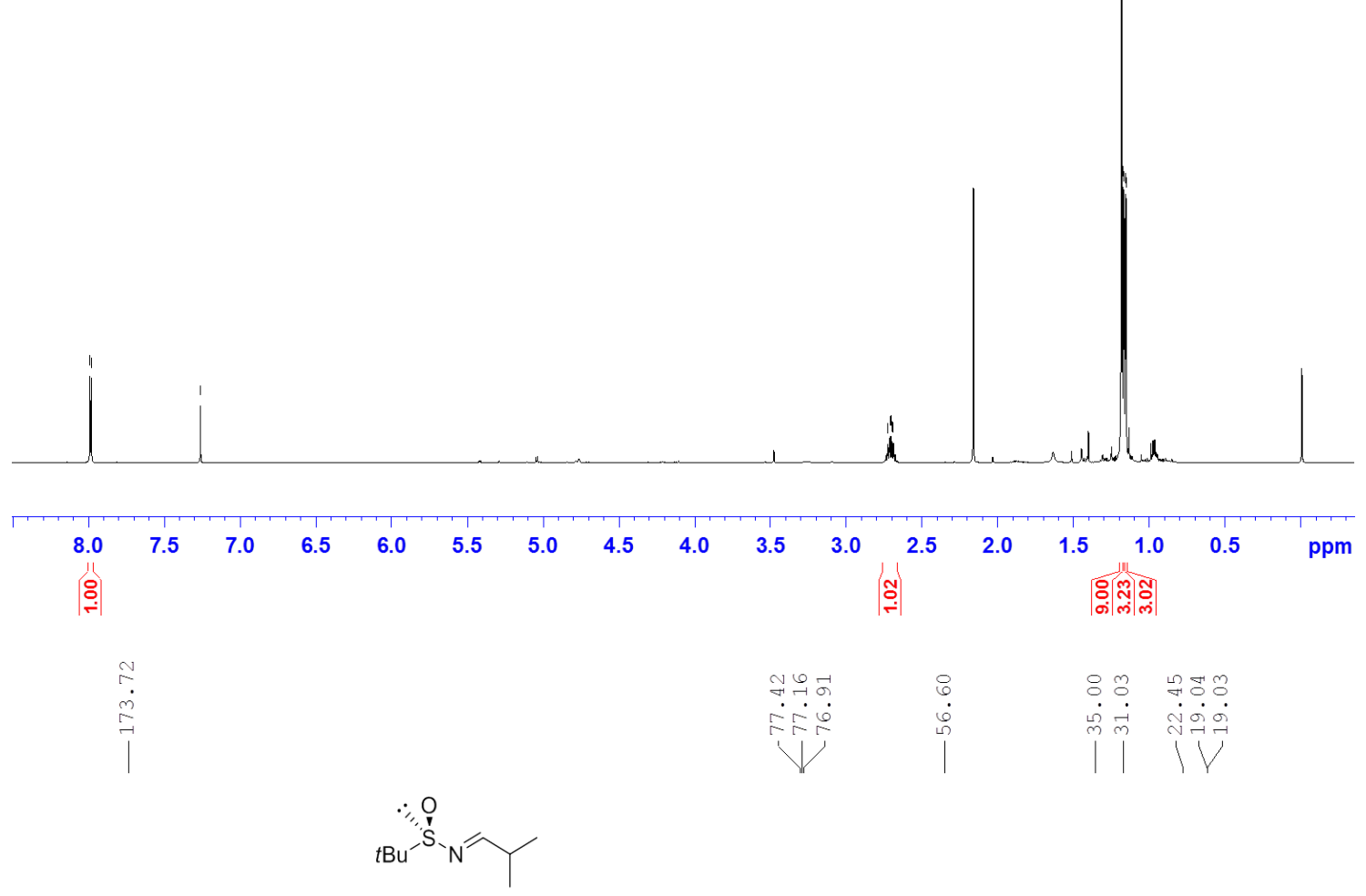

${ }^{13} \mathrm{C} \mathrm{NMR}\left(125 \mathrm{MHz}, \mathrm{CDCl}_{3}\right)$

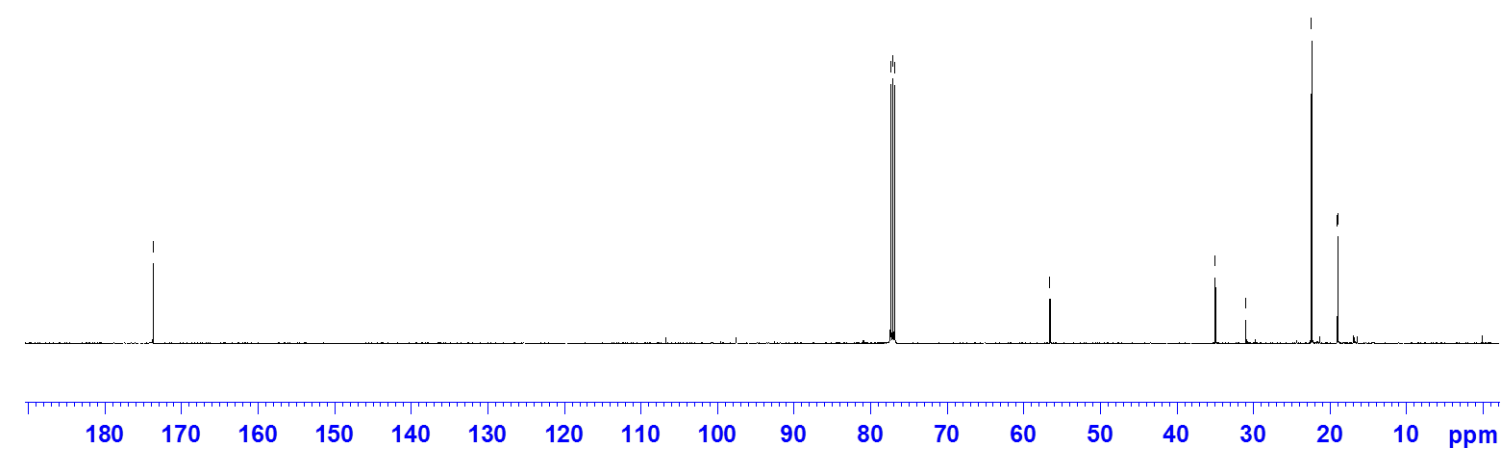


Methyl methyl(phenyl)phosphinate, rac-7.

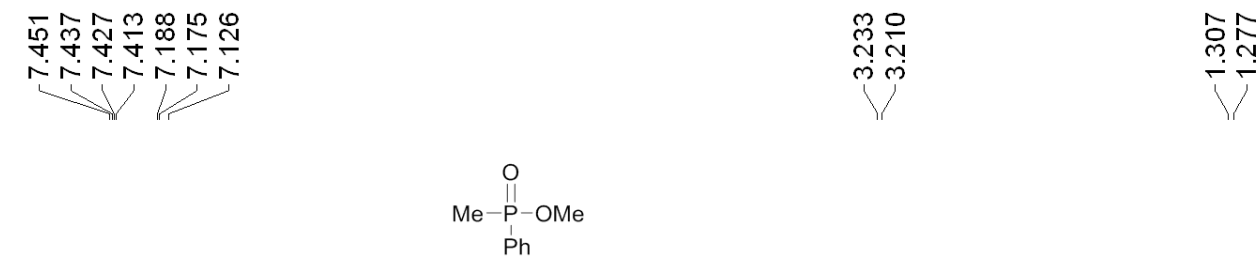

${ }^{1} \mathrm{H} \mathrm{NMR}\left(500 \mathrm{MHz}, \mathrm{CDCl}_{3}\right)$

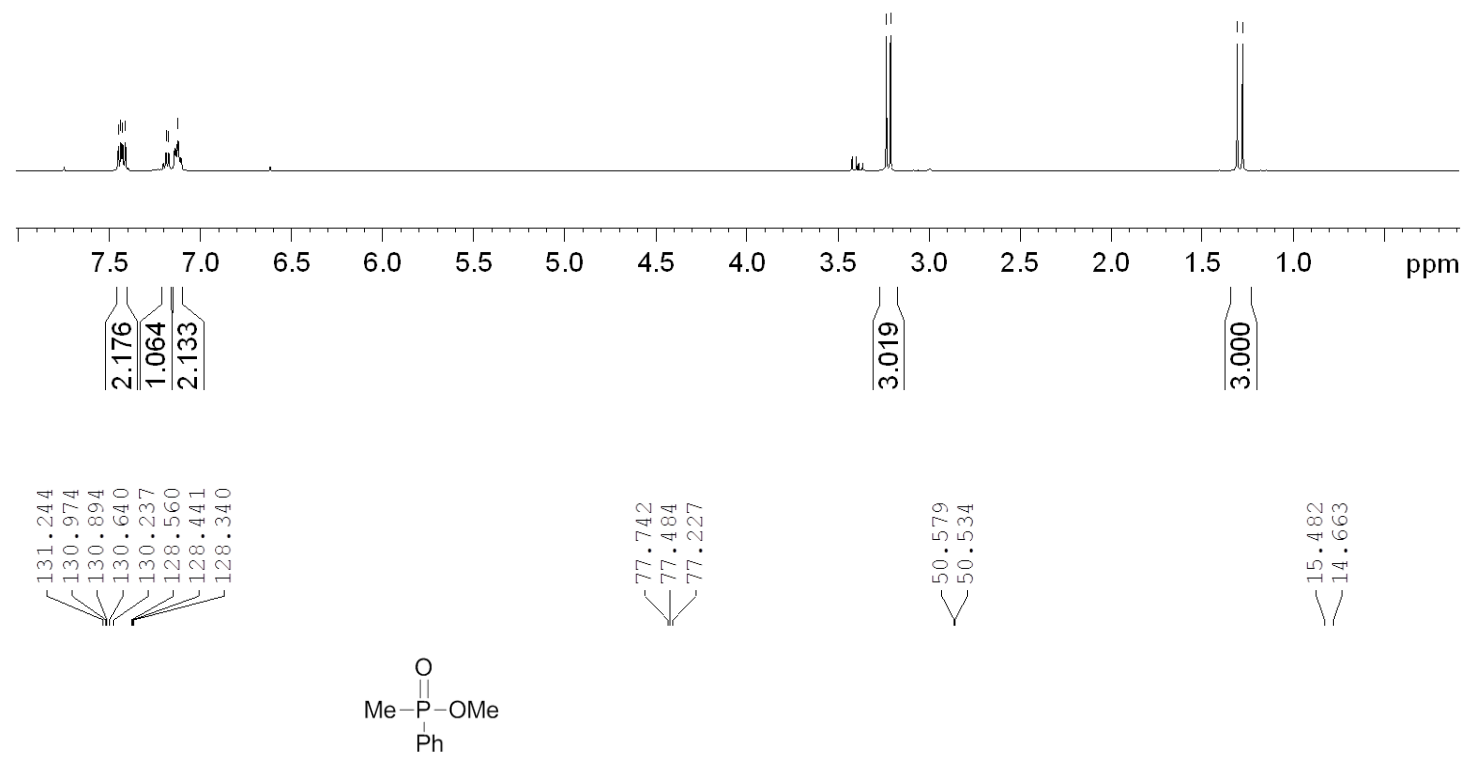

${ }^{13} \mathrm{C} \mathrm{NMR}\left(125 \mathrm{MHz}, \mathrm{CDCl}_{3}\right)$

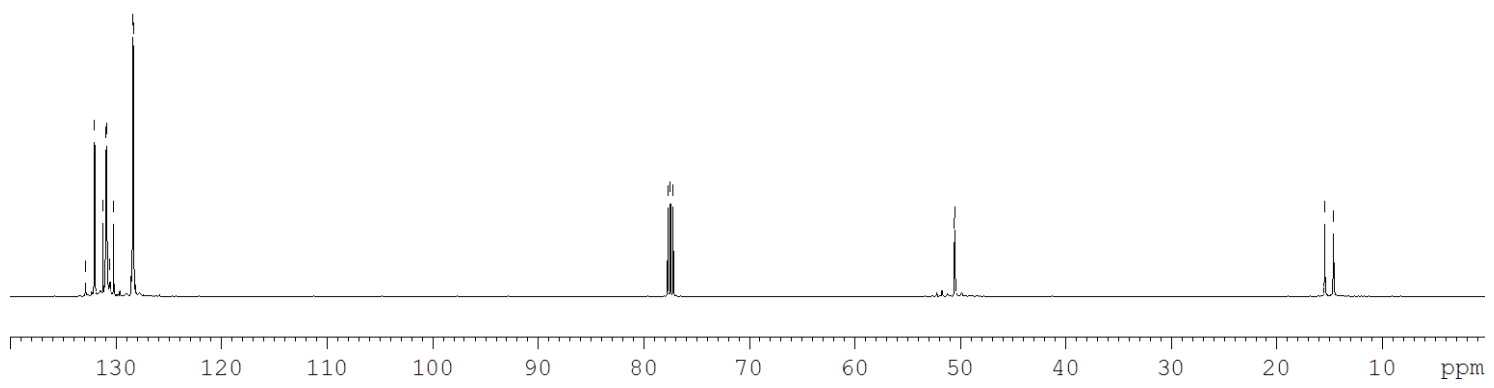




$$
\underset{\substack{\mathrm{O} \\ \mathrm{Me}-\mathrm{P}-\mathrm{OMe} \\ \mathrm{Ph}}}{\stackrel{2}{\mathrm{P}}}
$$

${ }^{31} \mathrm{P}$ NMR $\left(202 \mathrm{MHz}, \mathrm{CDCl}_{3}\right)$

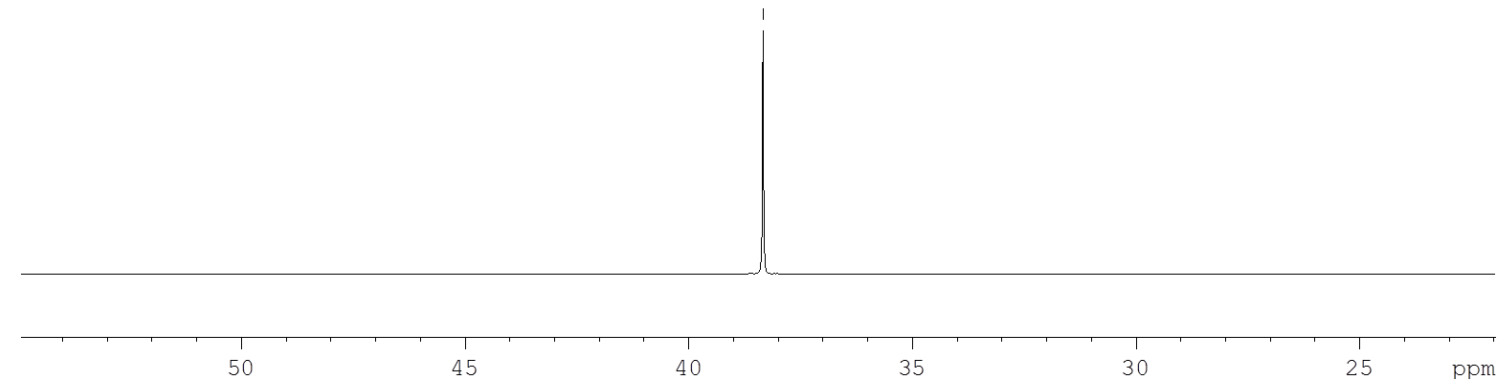

Methyl 2-(tert-Butylsulfinamide)-2-( $p$-methoxyphenyl)ethyl phenylphosphinate, $8(R, S, R)$.

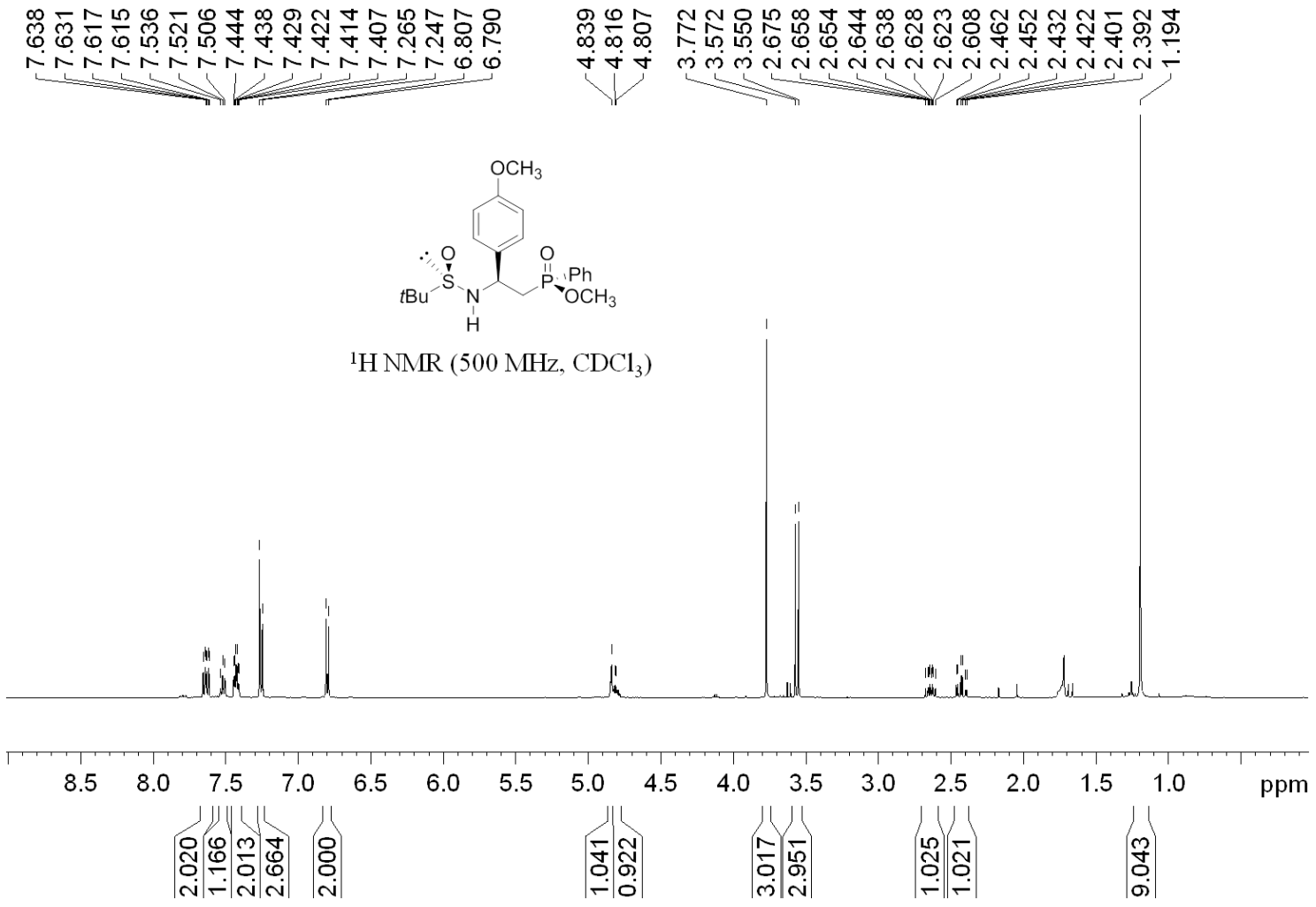




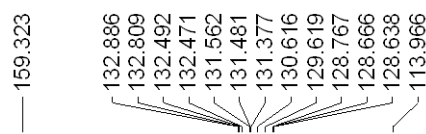

$$
\text { ST }
$$

${ }^{13} \mathrm{C} \mathrm{NMR}\left(125 \mathrm{MHz}, \mathrm{CDCl}_{3}\right)$
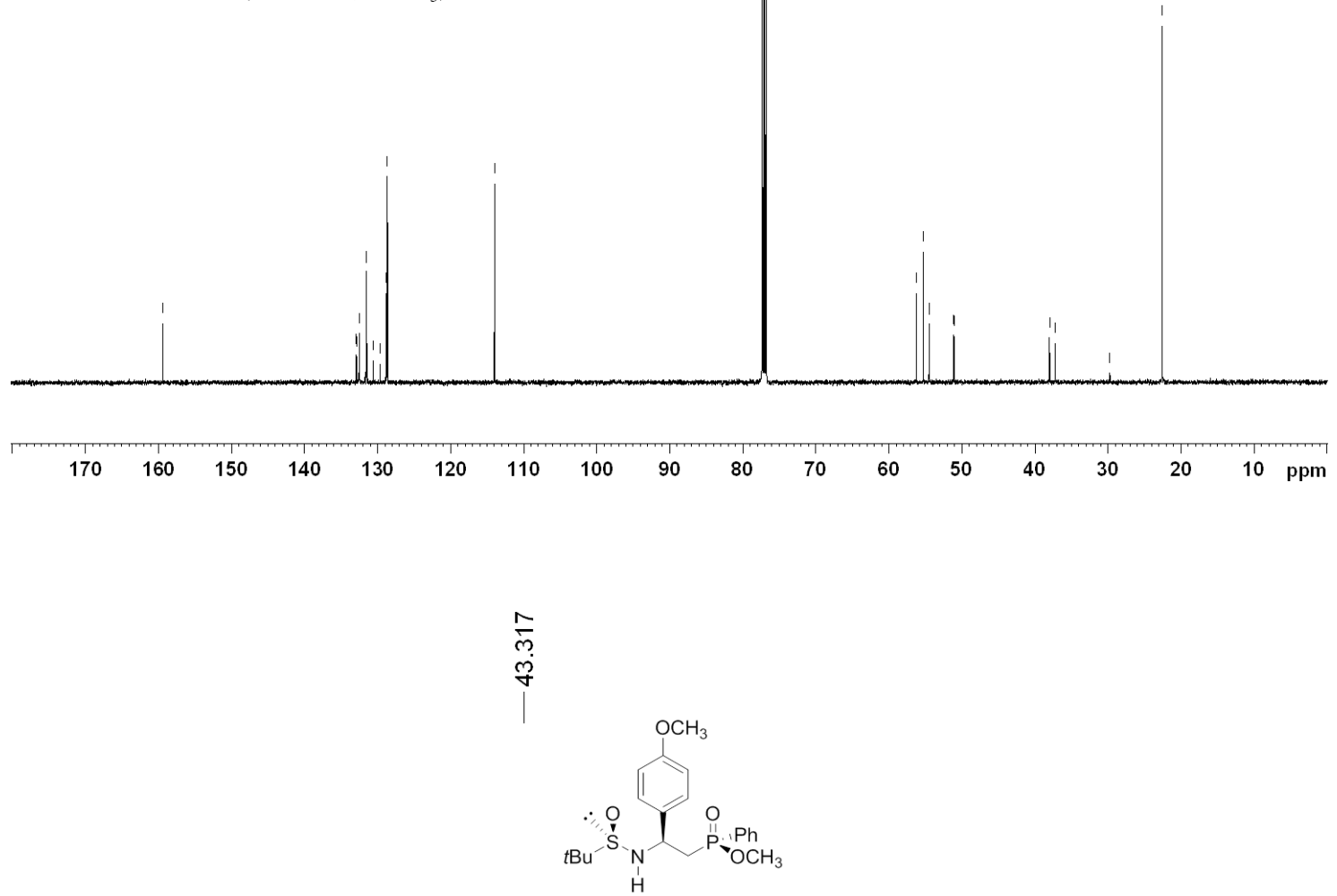

${ }^{31} \mathrm{PNMR}\left(202 \mathrm{MHz}, \mathrm{CDCl}_{3}\right.$ )

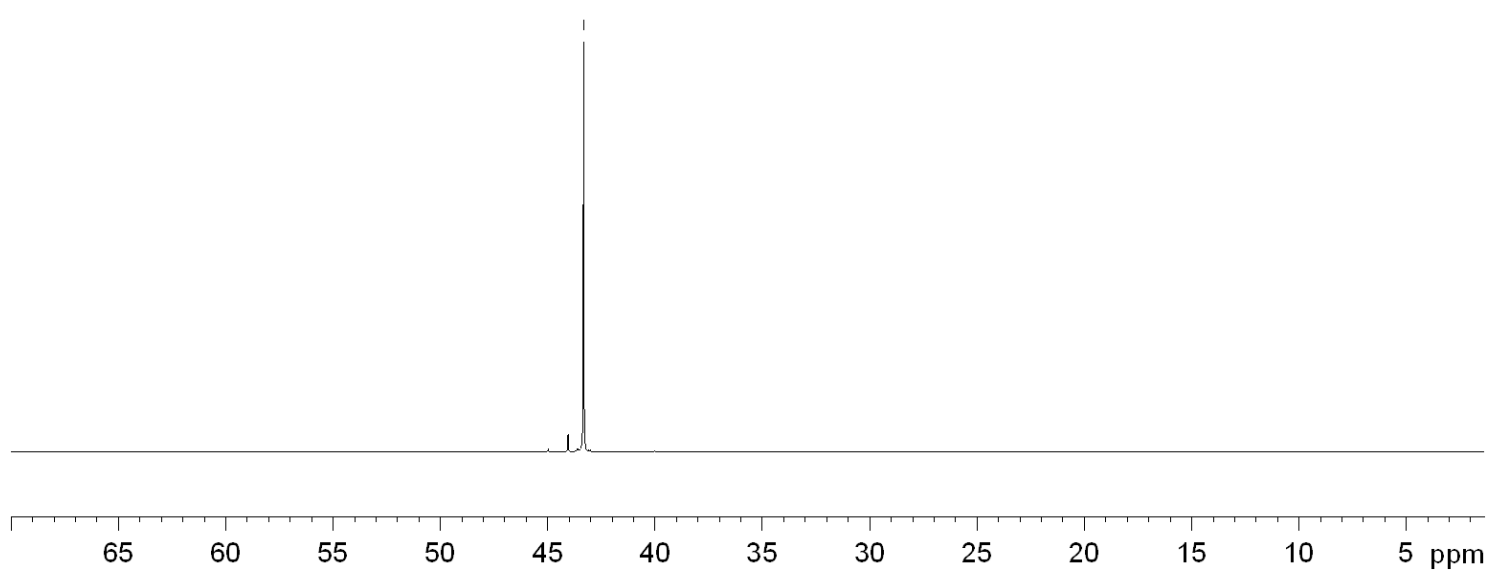




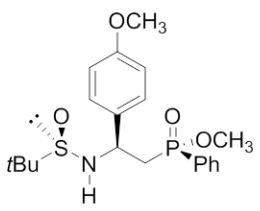

${ }^{1} \mathrm{H} \mathrm{NMR}\left(500 \mathrm{MHz}, \mathrm{CDCl}_{3}\right)$
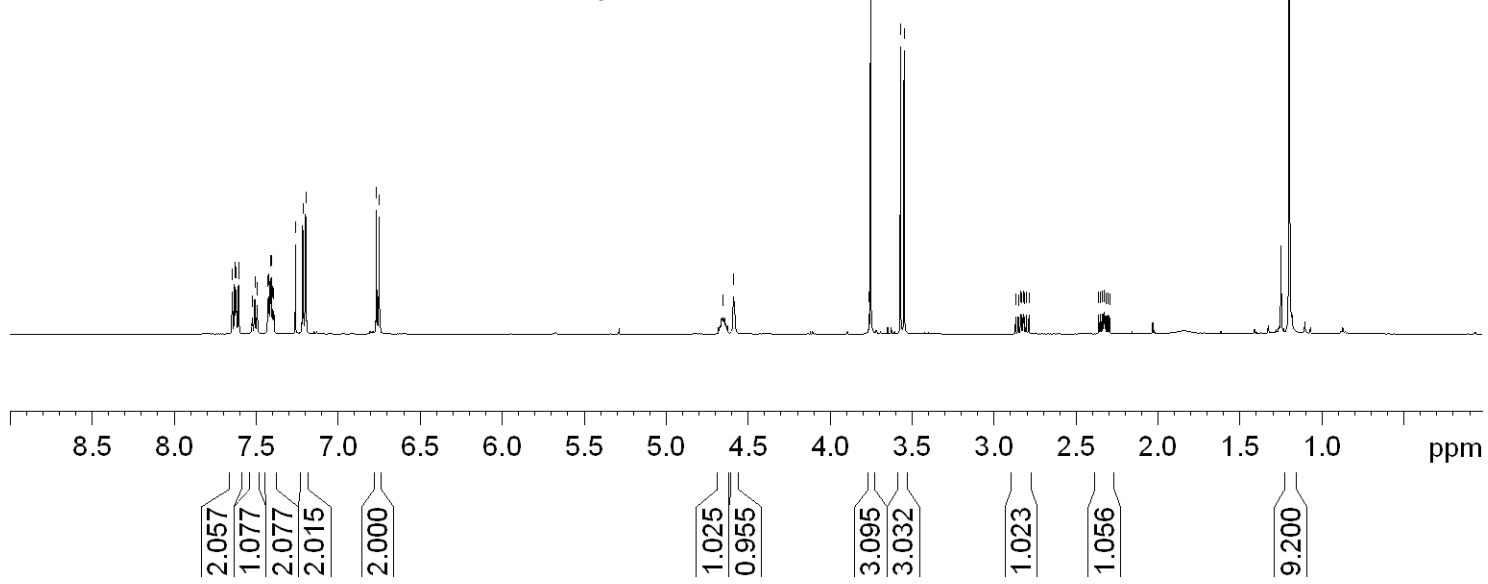

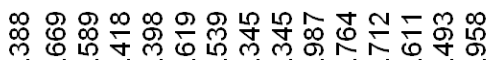

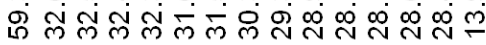

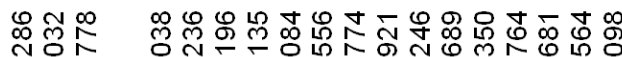

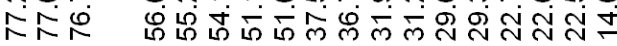
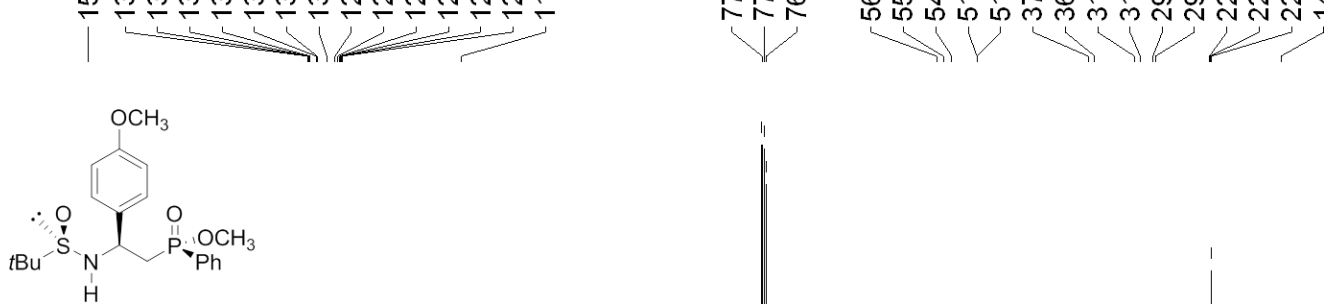

${ }^{13} \mathrm{CNMR}\left(125 \mathrm{MHz}, \mathrm{CDCl}_{3}\right)$

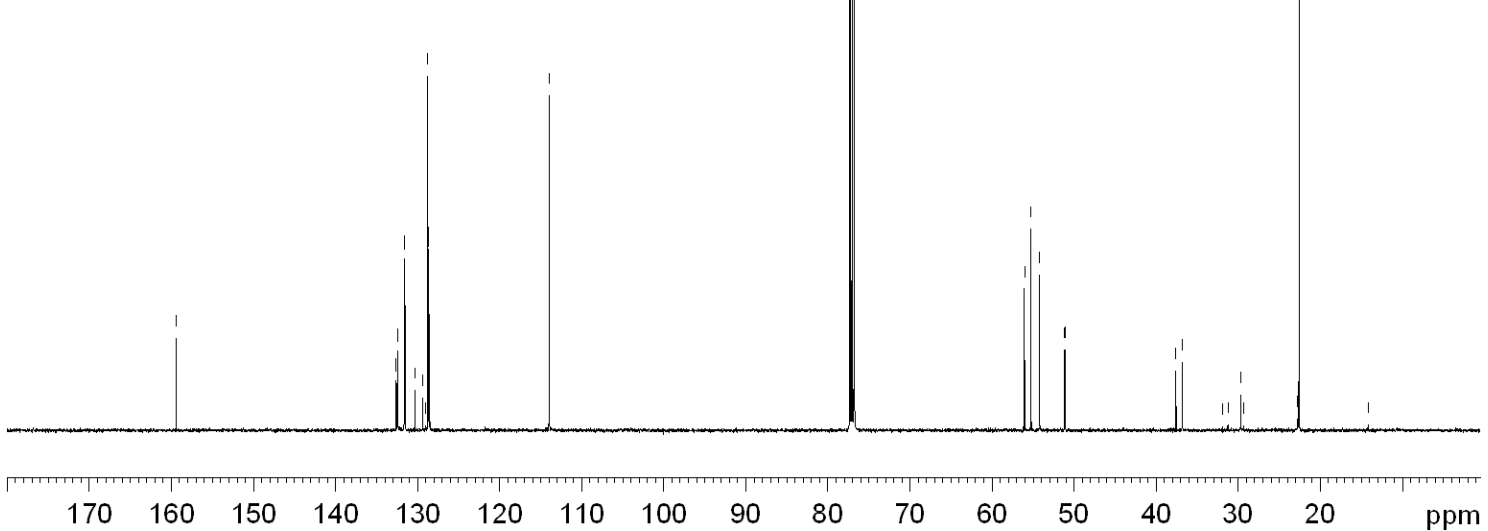




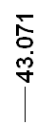

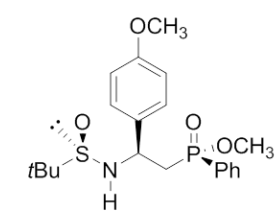

${ }^{31} \mathrm{PNMR}\left(202 \mathrm{MHz}, \mathrm{CDCl}_{3}\right)$

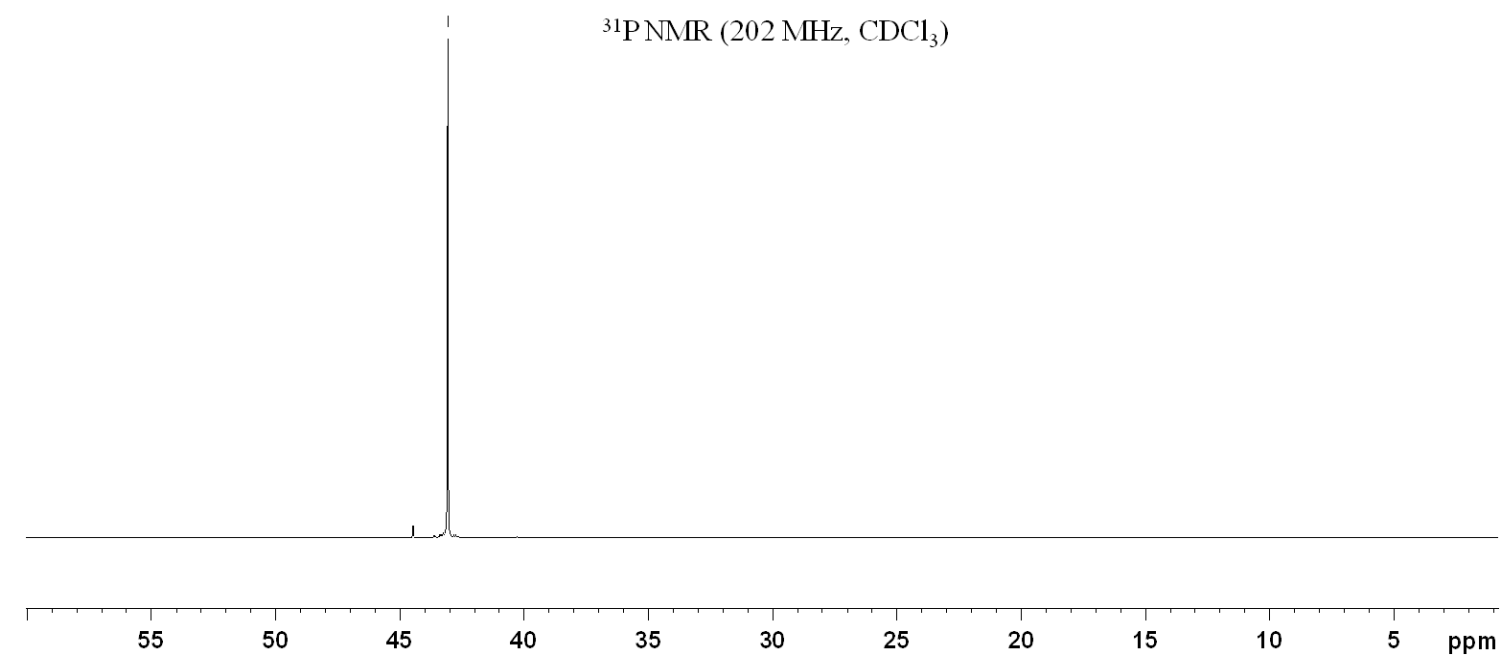

\section{Methyl 2-(tert-butylsulfinamide)-2-[3,5-bis(trifluoromethyl)phenyl]ethyl}

phenylphosphinate, $9(R, S, R)$
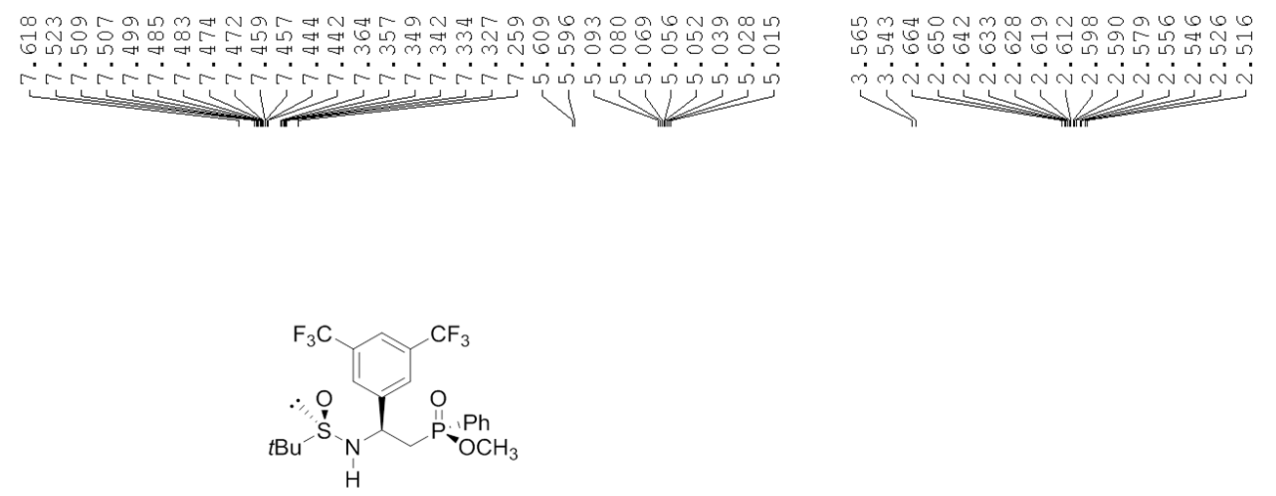

${ }^{1} \mathrm{H} \mathrm{NMR}\left(500 \mathrm{MHz}, \mathrm{CDCl}_{3}\right)$

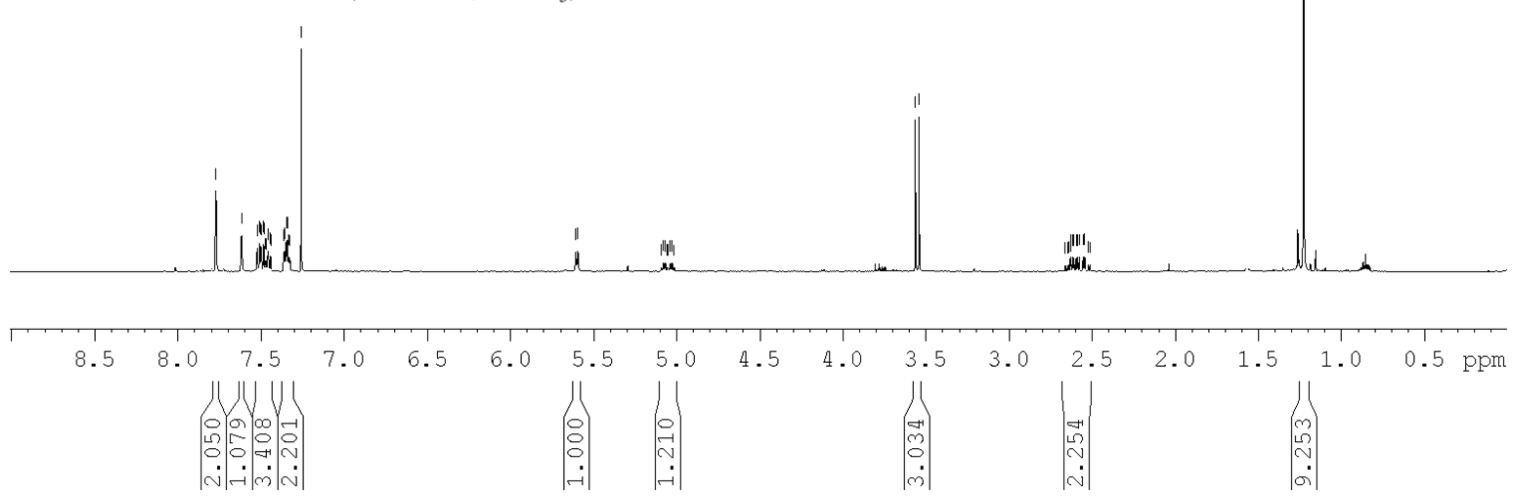




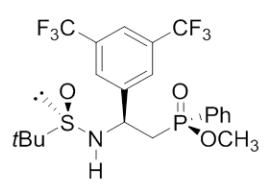

${ }^{13} \mathrm{CNMR}\left(125 \mathrm{MHz}, \mathrm{CDCl}_{3}\right)$
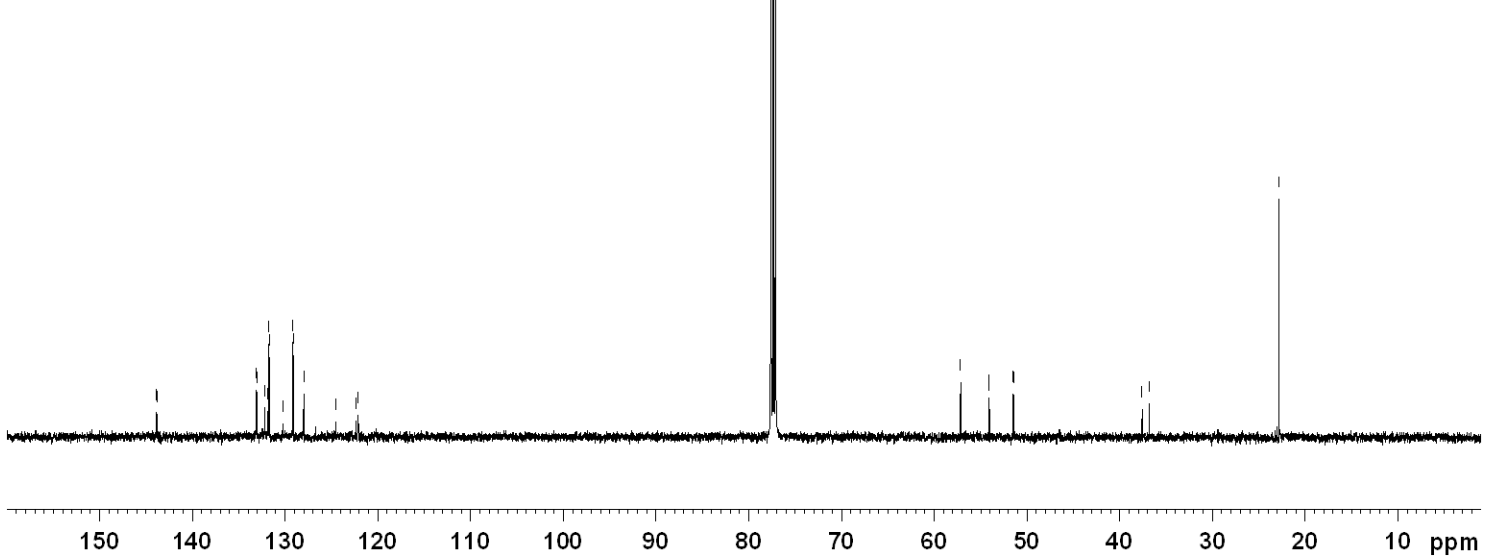

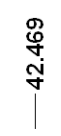

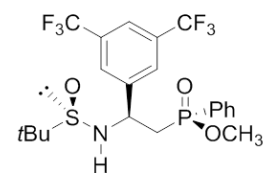

${ }^{31} \mathrm{PNMR}\left(202 \mathrm{MHz}, \mathrm{CDCl}_{3}\right)$

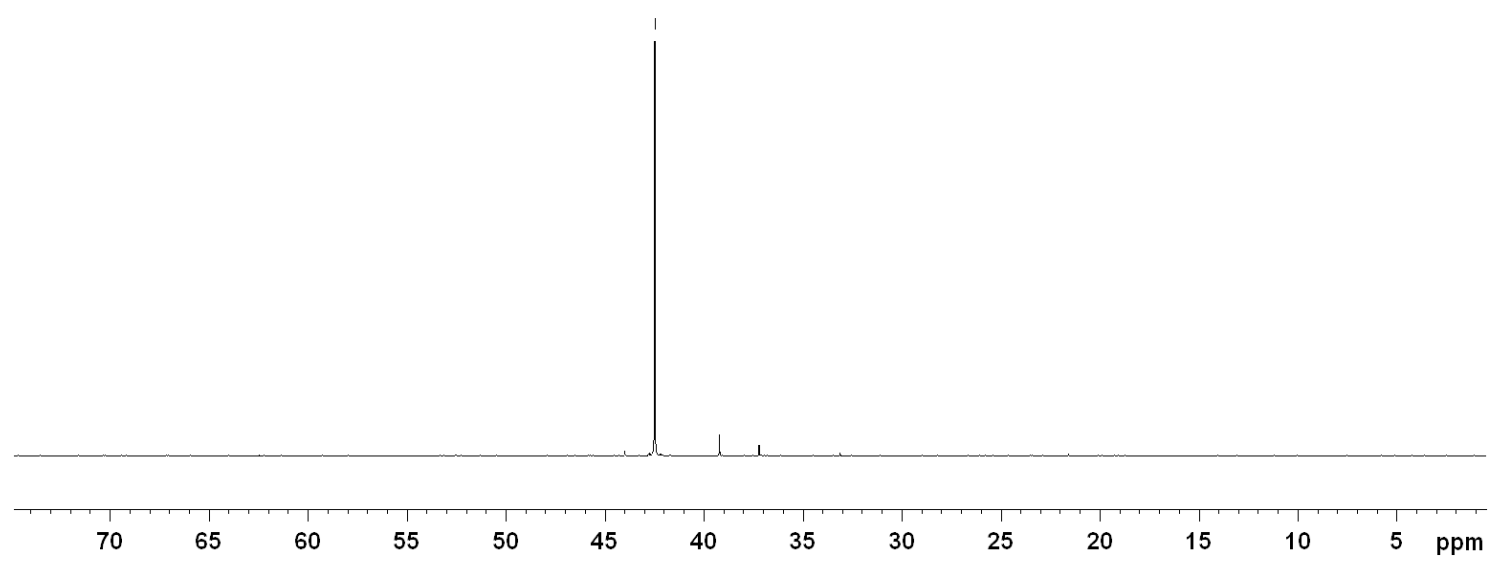


Methyl 2-(tert-butylsulfinamide)-2-[3,5-bis(trifluoromethyl)phenyl]ethyl phenylphosphinate, $9(R, S, S)$.

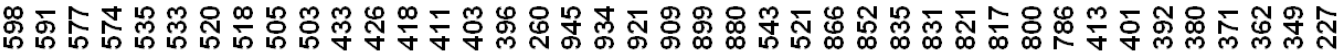
NiN

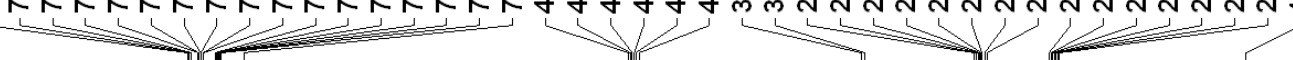

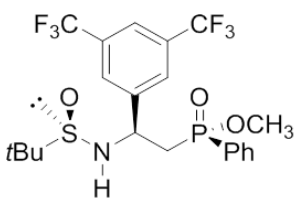

${ }^{1} \mathrm{H} \mathrm{NMR}\left(500 \mathrm{MHz}, \mathrm{CDCl}_{3}\right)$

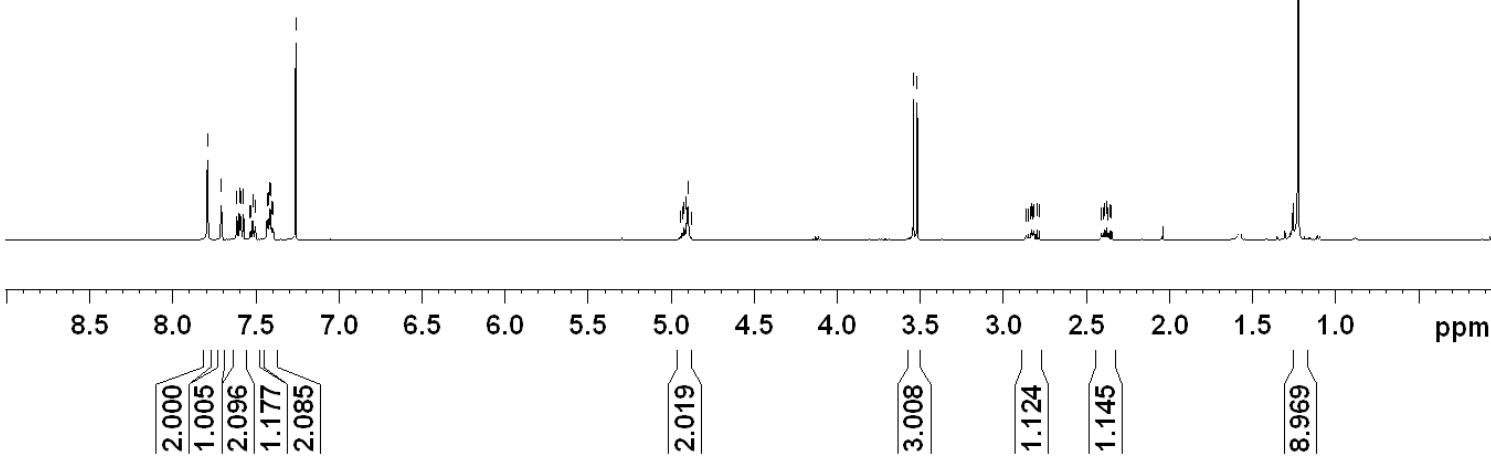

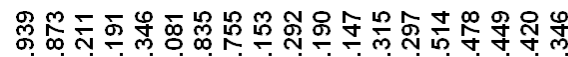

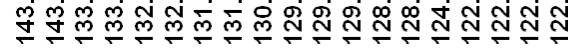

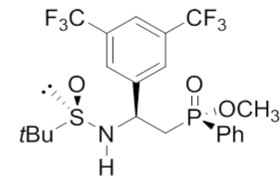

${ }^{13} \mathrm{C} \mathrm{NMR}\left(125 \mathrm{MHz}, \mathrm{CDCl}_{3}\right)$

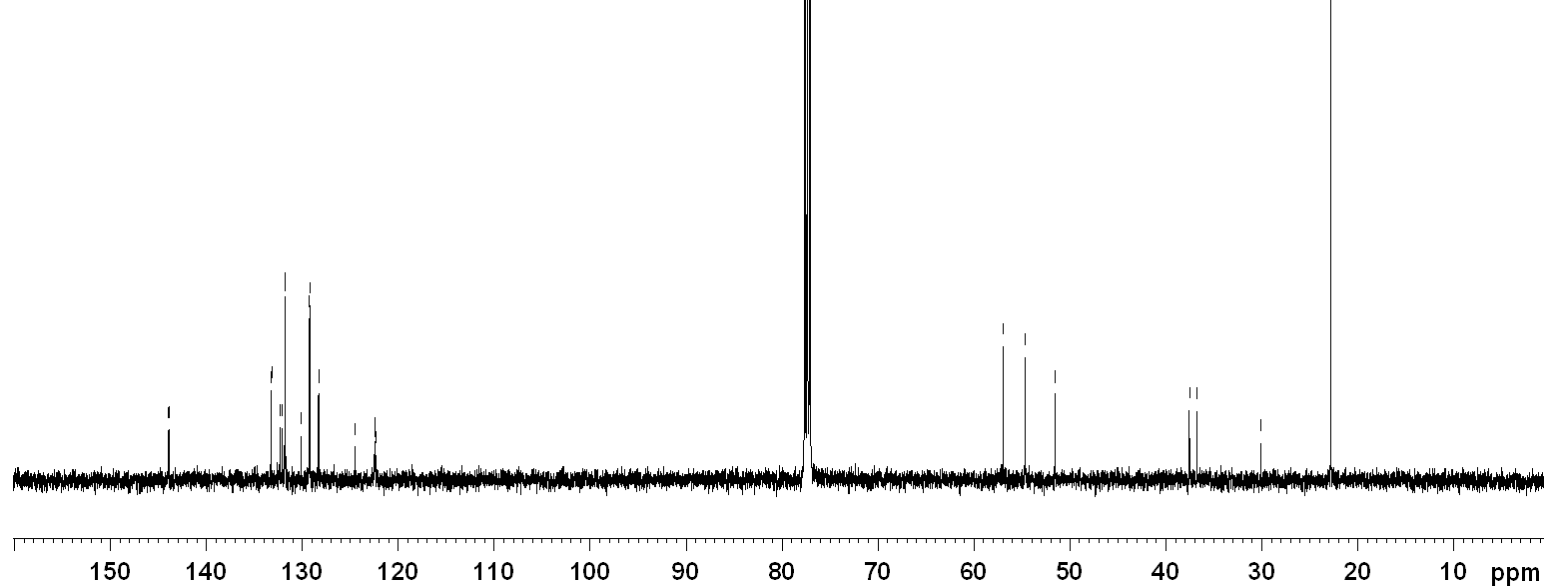




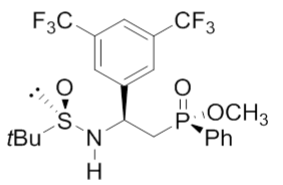

${ }^{31} \mathrm{PNMR}\left(202 \mathrm{MHz}, \mathrm{CDCl}_{3}\right)$

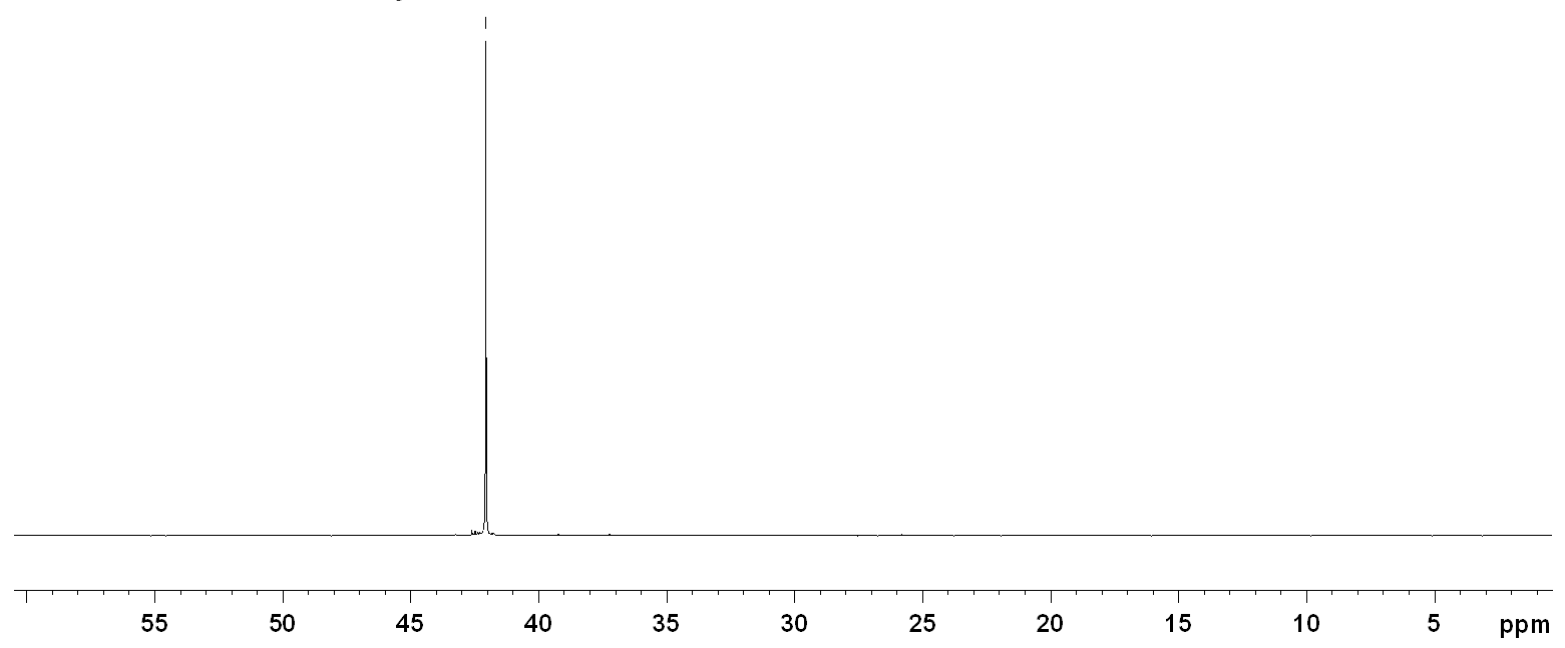

Methyl 2-(tert-butylsulfinamide)-2-(2'-naphthyl)ethyl phenylphosphinate, $10(R, S, R)$, SulPhos

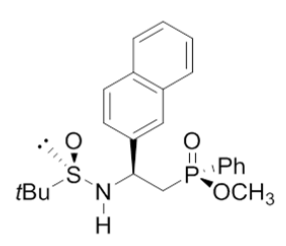

${ }^{1} \mathrm{H} \mathrm{NMR}\left(500 \mathrm{MHz}, \mathrm{CDCl}_{3}\right)$

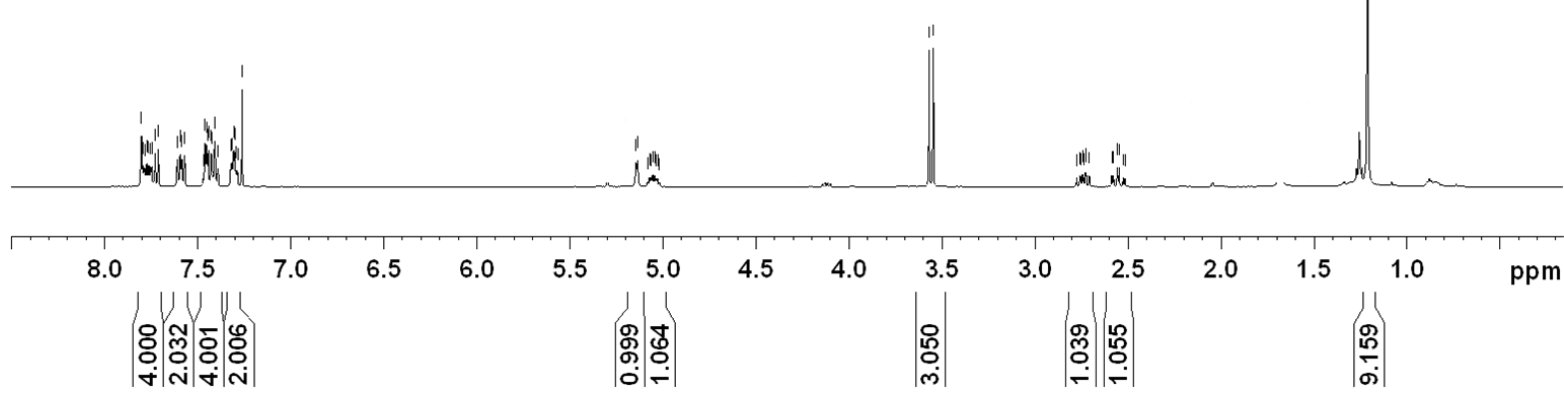




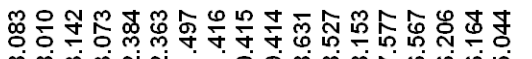

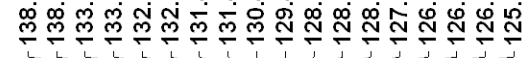

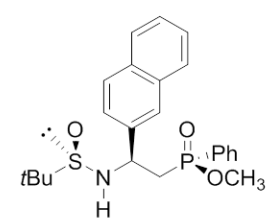

${ }^{13} \mathrm{C} \mathrm{NMR}\left(125 \mathrm{MHz}, \mathrm{CDCl}_{3}\right.$ )

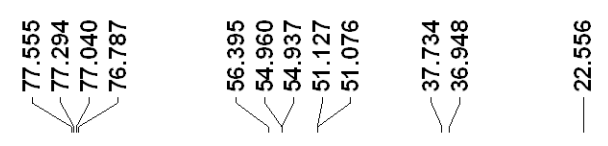
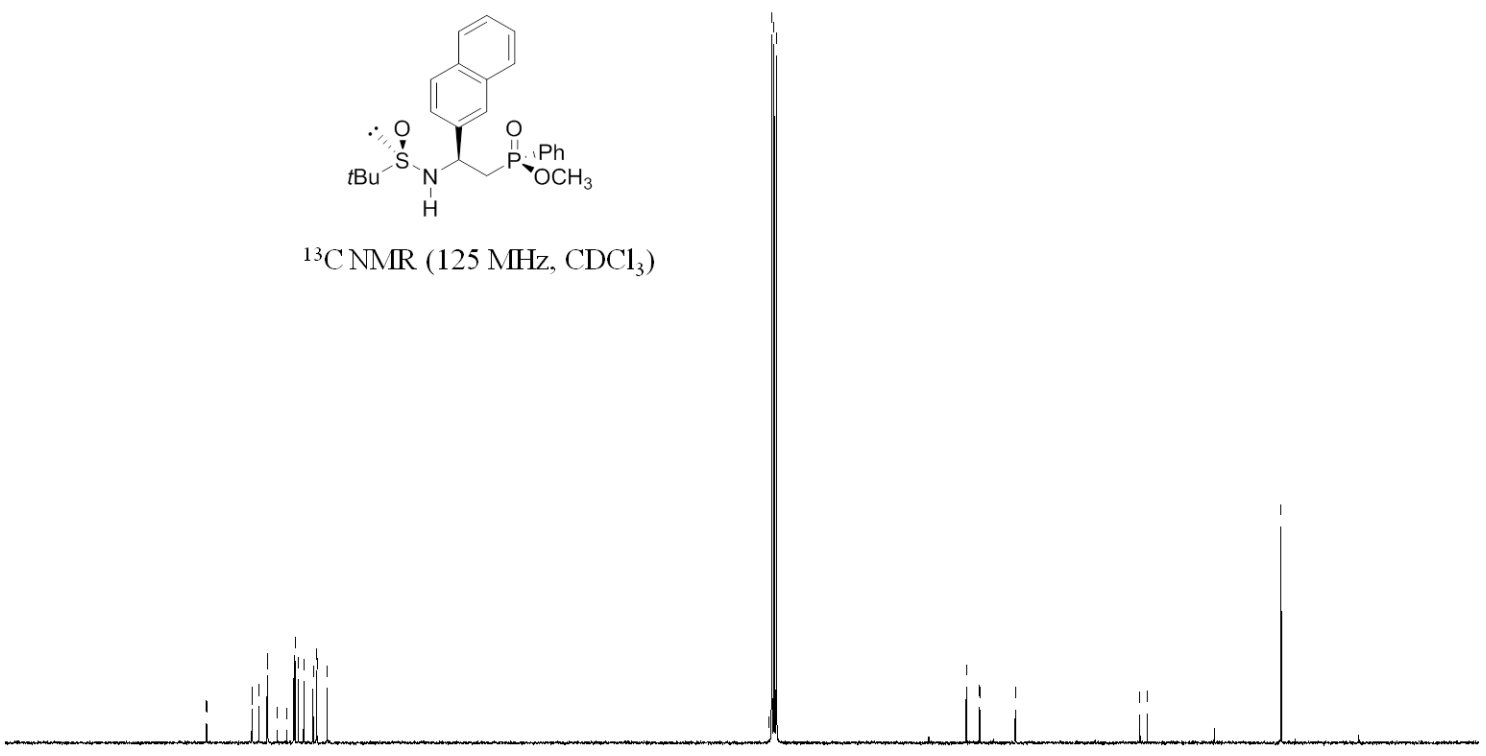

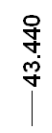

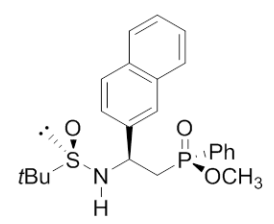

${ }^{31} \mathrm{P} \mathrm{NMR}\left(202 \mathrm{MHz}, \mathrm{CDCl}_{3}\right)$

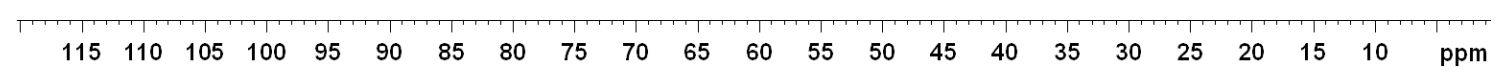

33 
Methyl 2-(tert-butylsulfinamide)-2-(2'-naphthyl)ethyl phenylphosphinate, $10(R, S, S)$

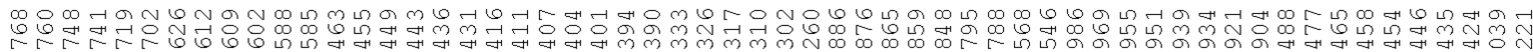
-

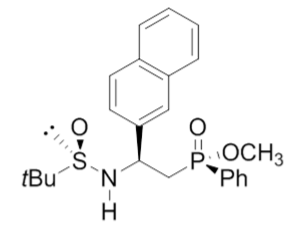

${ }^{1} \mathrm{H} \mathrm{NMR}\left(500 \mathrm{MHz}, \mathrm{CDCl}_{3}\right)$
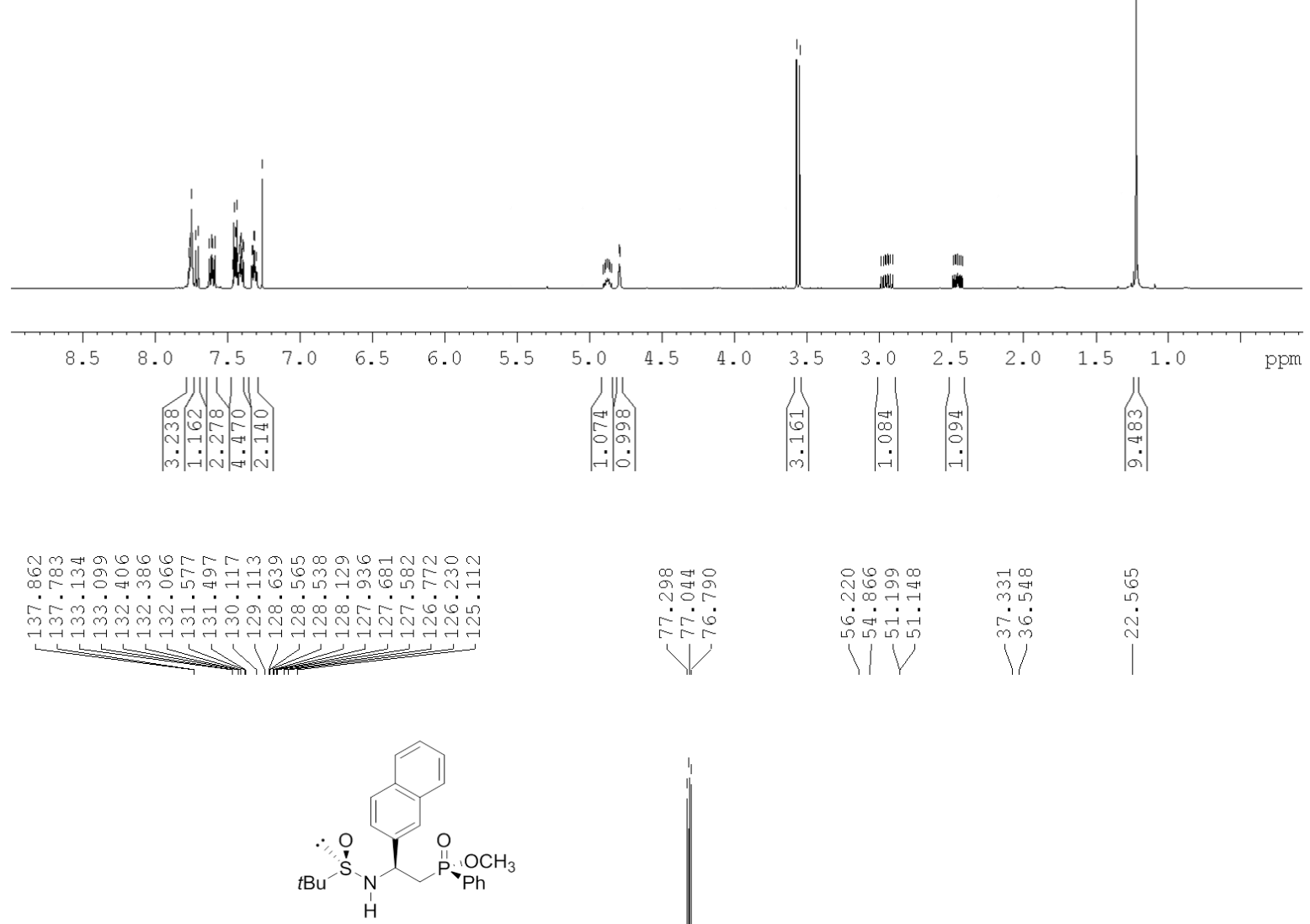

${ }^{13} \mathrm{C} \mathrm{NMR}\left(125 \mathrm{MHz}, \mathrm{CDCl}_{3}\right)$

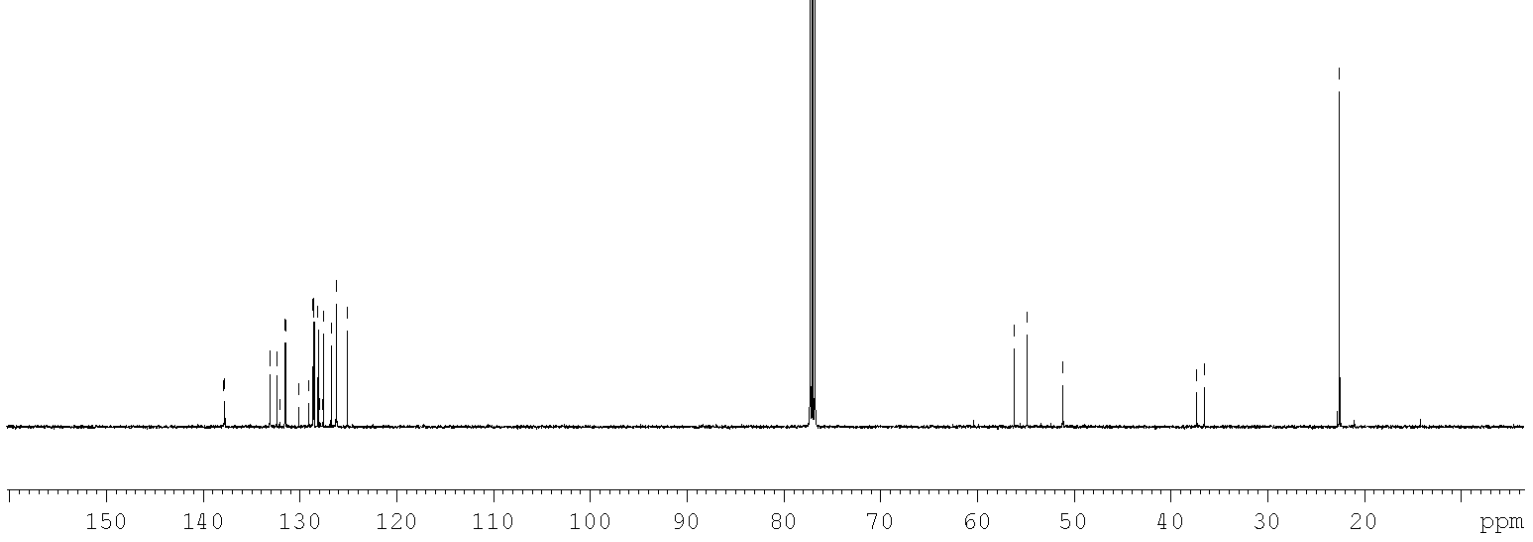




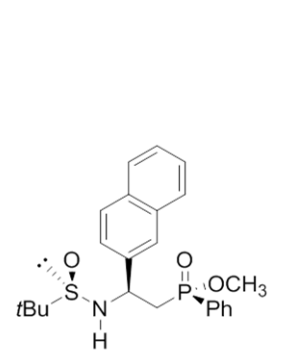

${ }^{31} \mathrm{PNMR}\left(202 \mathrm{MHz}, \mathrm{CDCl}_{3}\right)$

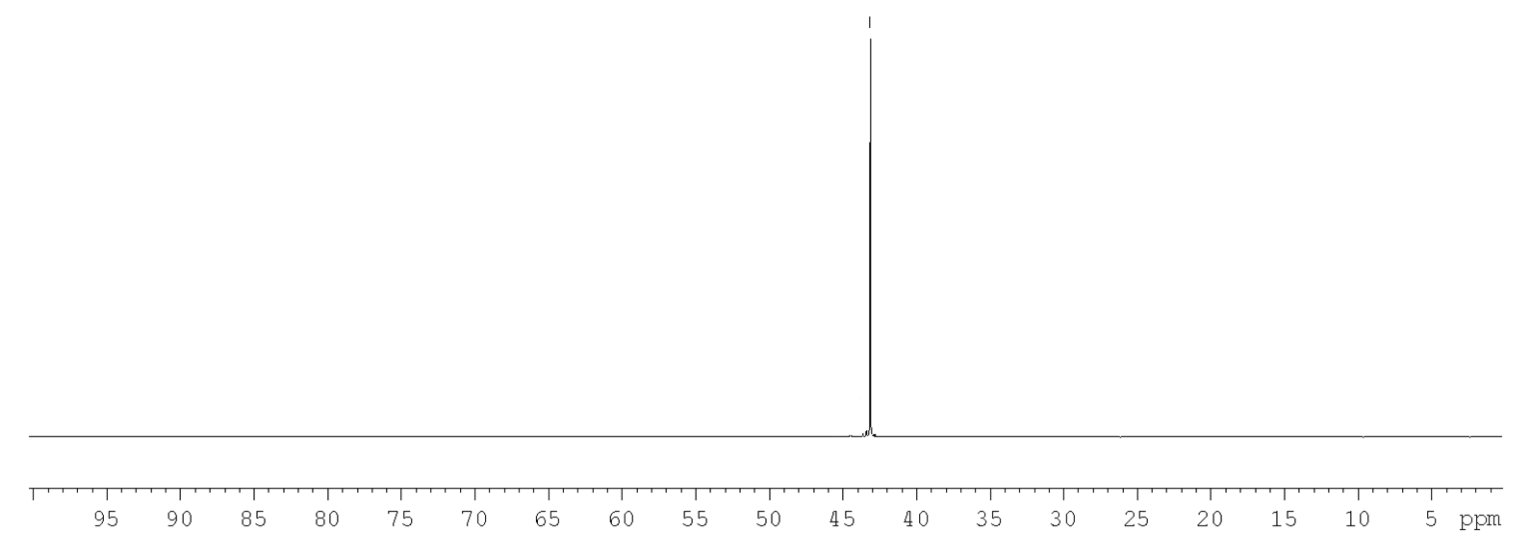

Methyl 2-(tert-butylsulfinamide)-2-(ferrocenyl)ethyl phenylphosphinate, $11(R, S, R)$.

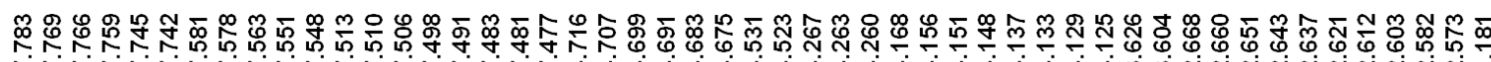

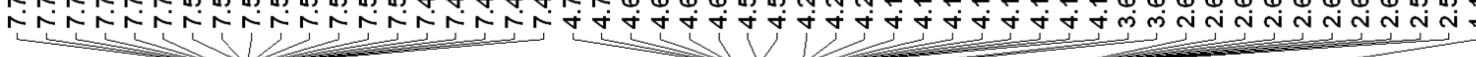

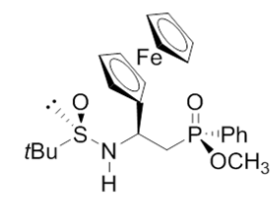

${ }^{1} \mathrm{H} \mathrm{NMR}\left(500 \mathrm{MHz}, \mathrm{CDCl}_{3}\right)$

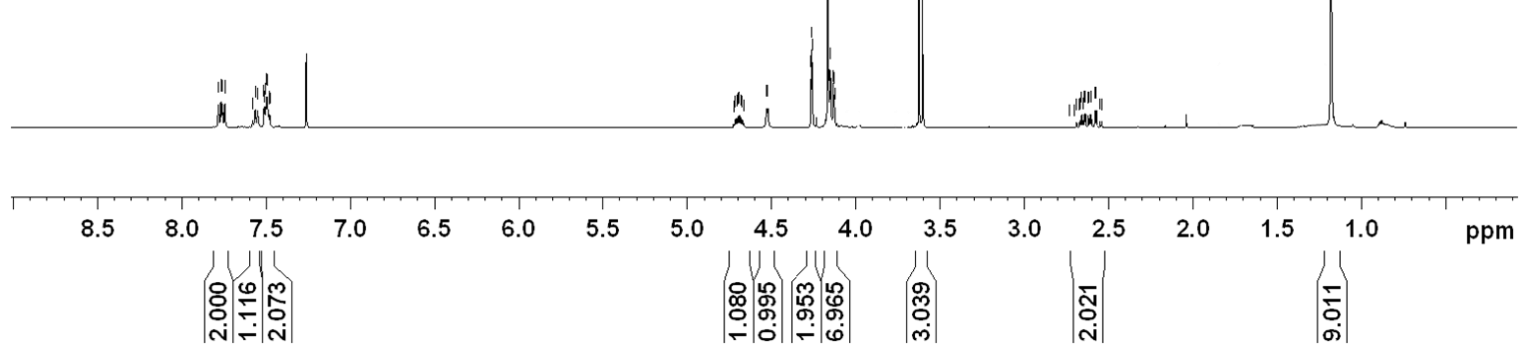




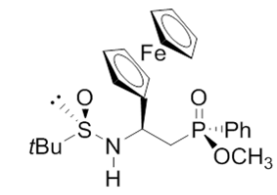

${ }^{1} \mathrm{H} \mathrm{NMR}\left(500 \mathrm{MHz}, \mathrm{CDCl}_{3}\right)$

i象 $\stackrel{1}{1}$
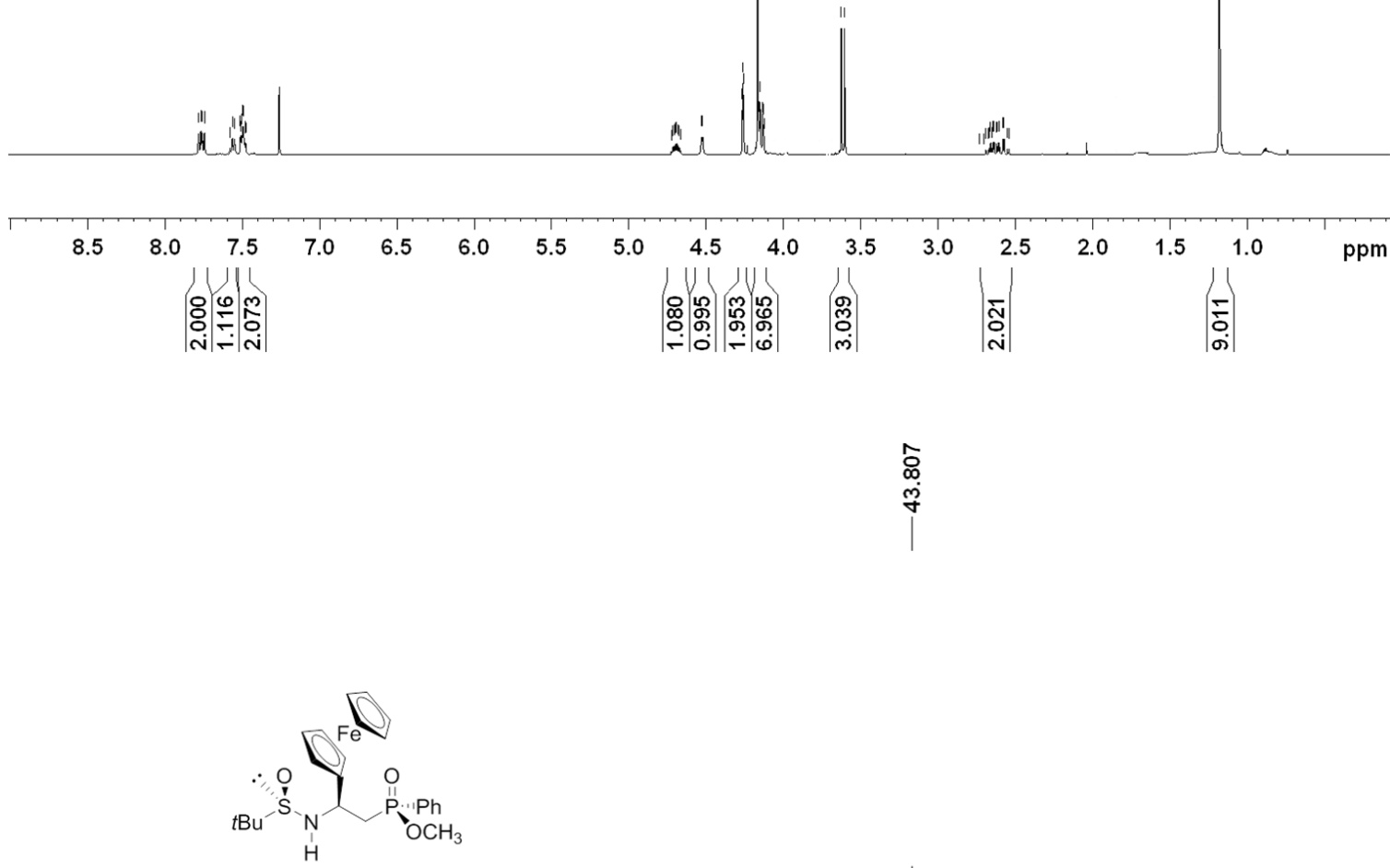

${ }^{31} \mathrm{PNMR}\left(202 \mathrm{MHz}, \mathrm{CDCl}_{3}\right)$ 
Methyl 2-(tert-butylsulfinamide)-2-(isopropyl)ethyl phenylphosphinate, $12(R, S, R)$.

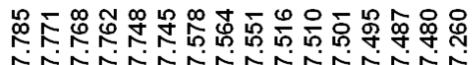

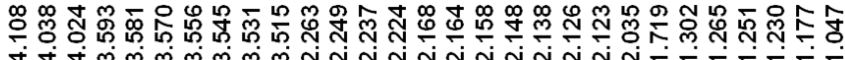

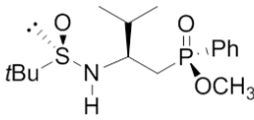

${ }^{1} \mathrm{H} \mathrm{NMR}\left(500 \mathrm{MHz}, \mathrm{CDCl}_{3}\right)$
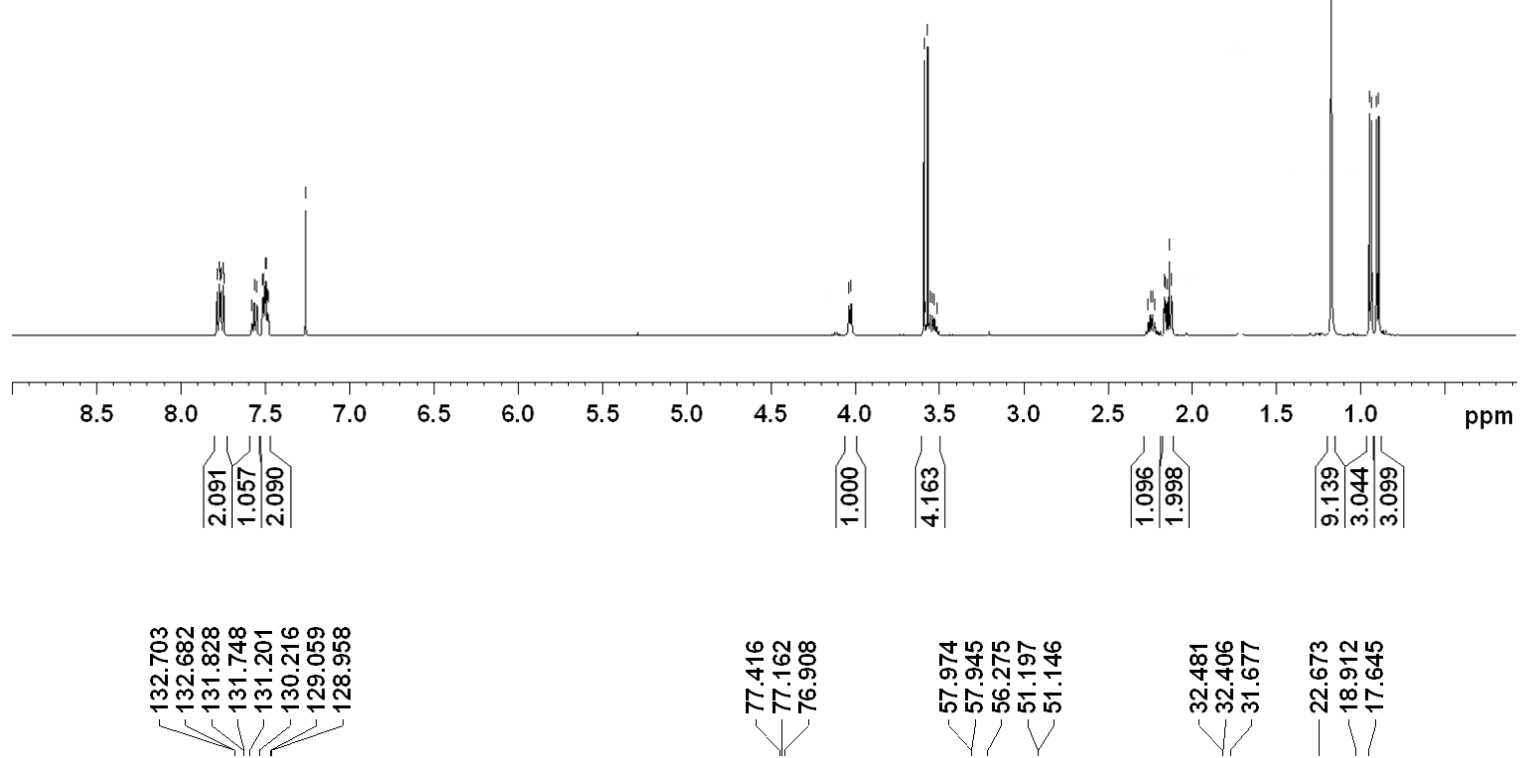

능ำ

乩战的的

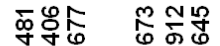

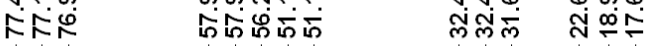

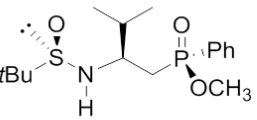

${ }^{13} \mathrm{CNMR}\left(125 \mathrm{MHz}, \mathrm{CDCl}_{3}\right)$

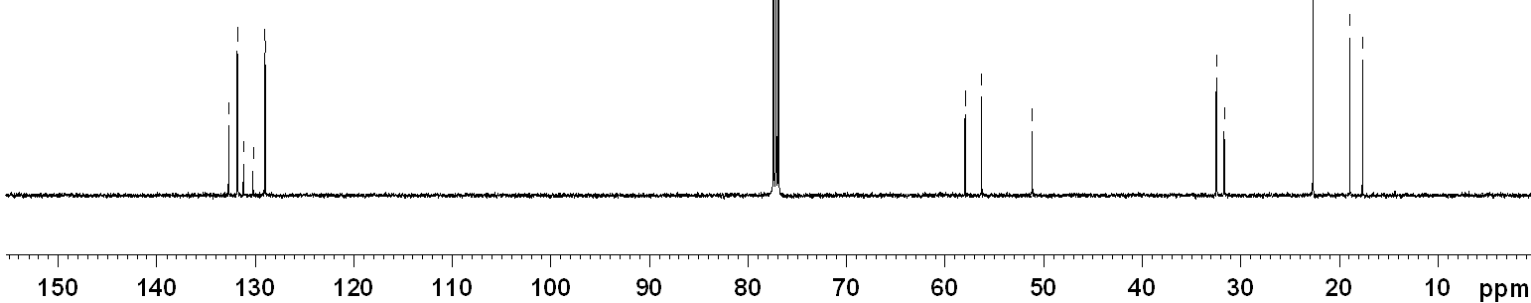




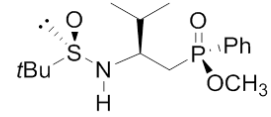

${ }^{31} \mathrm{P} \mathrm{NMR}\left(202 \mathrm{MHz}, \mathrm{CDCl}_{3}\right)$

$110 \quad 100$

90

80

70

60

50

40

30

20

$10 \mathrm{ppm}$

(R,S)-2-(tert-Butilsulfinamide)-2-(2'-naphthyl)ethyl diphenyl phosphine oxide, 17(R,S).

品题

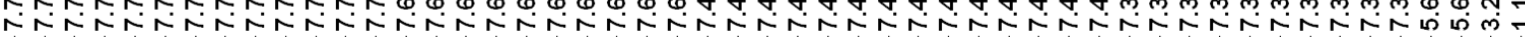

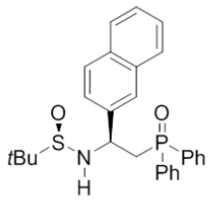

${ }^{1} \mathrm{H} \mathrm{NMR}\left(500 \mathrm{MHz}, \mathrm{CDCl}_{3}\right.$ )

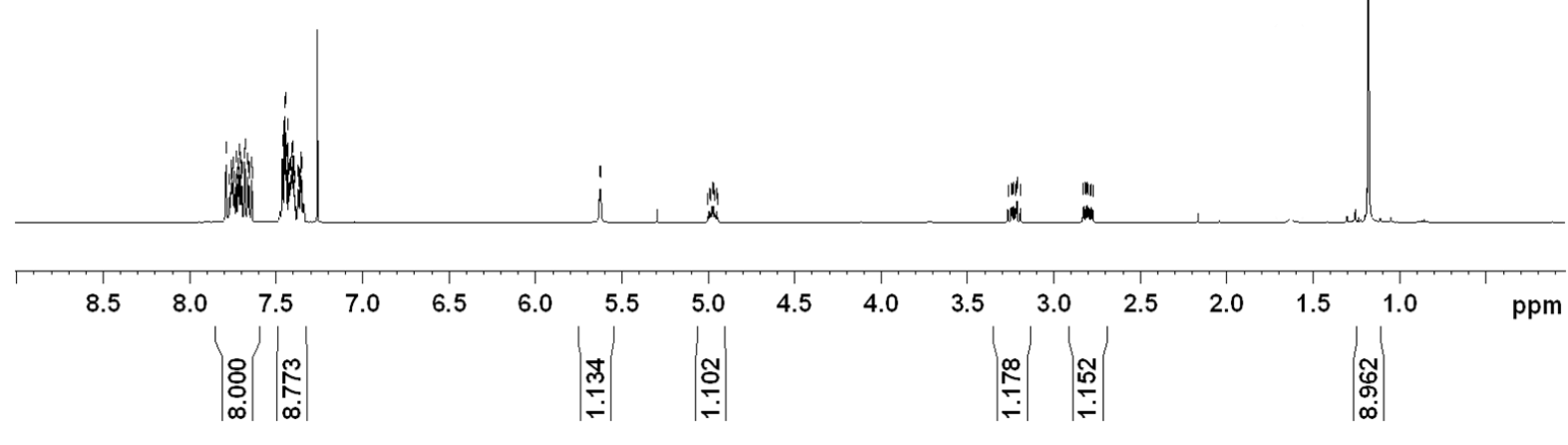




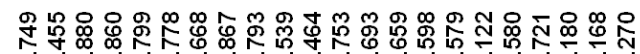

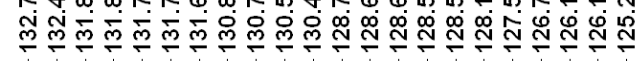

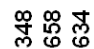

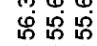

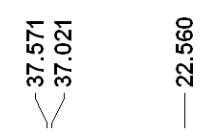

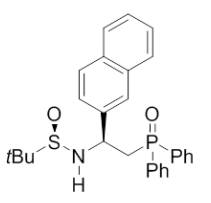

${ }^{13} \mathrm{CNMR}\left(125 \mathrm{MHz}, \mathrm{CDCl}_{3}\right)$
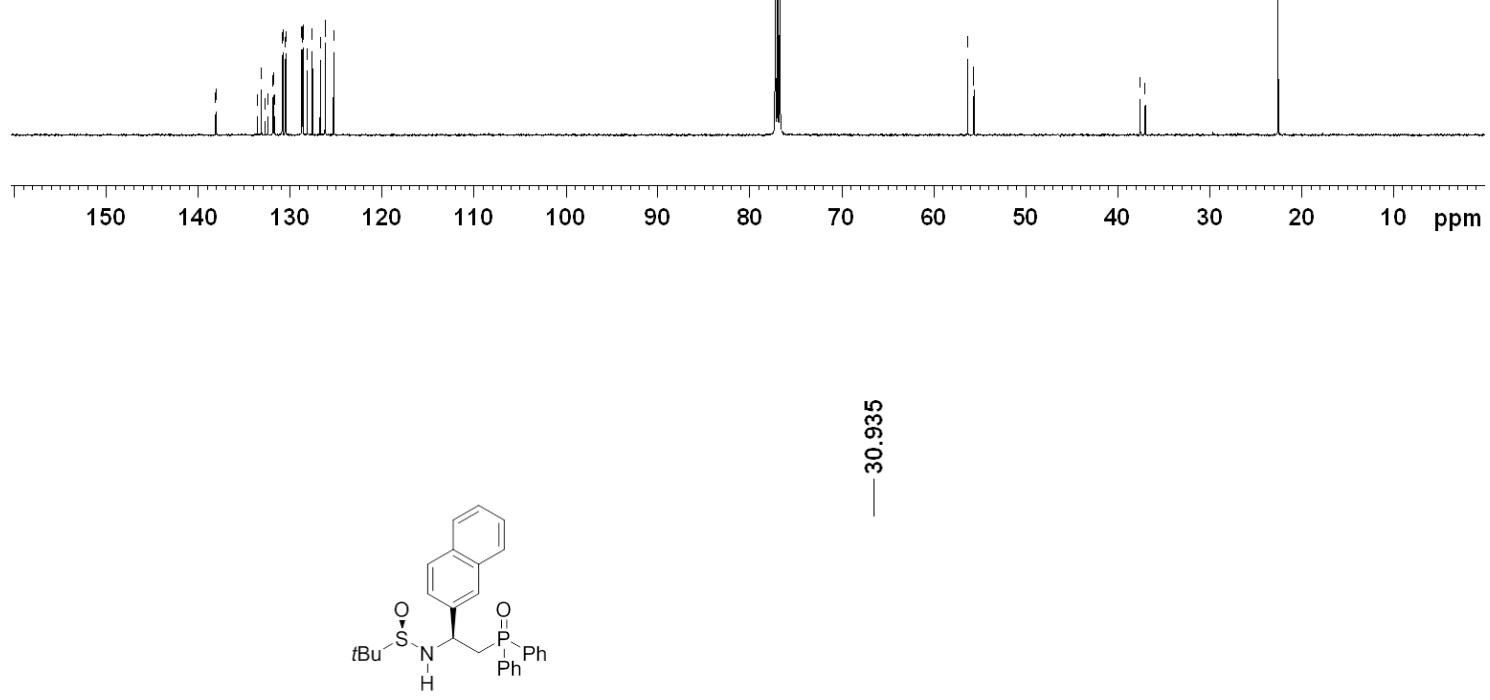

${ }^{31} \mathrm{PNMR}\left(202 \mathrm{MHz}, \mathrm{CDCl}_{3}\right)$

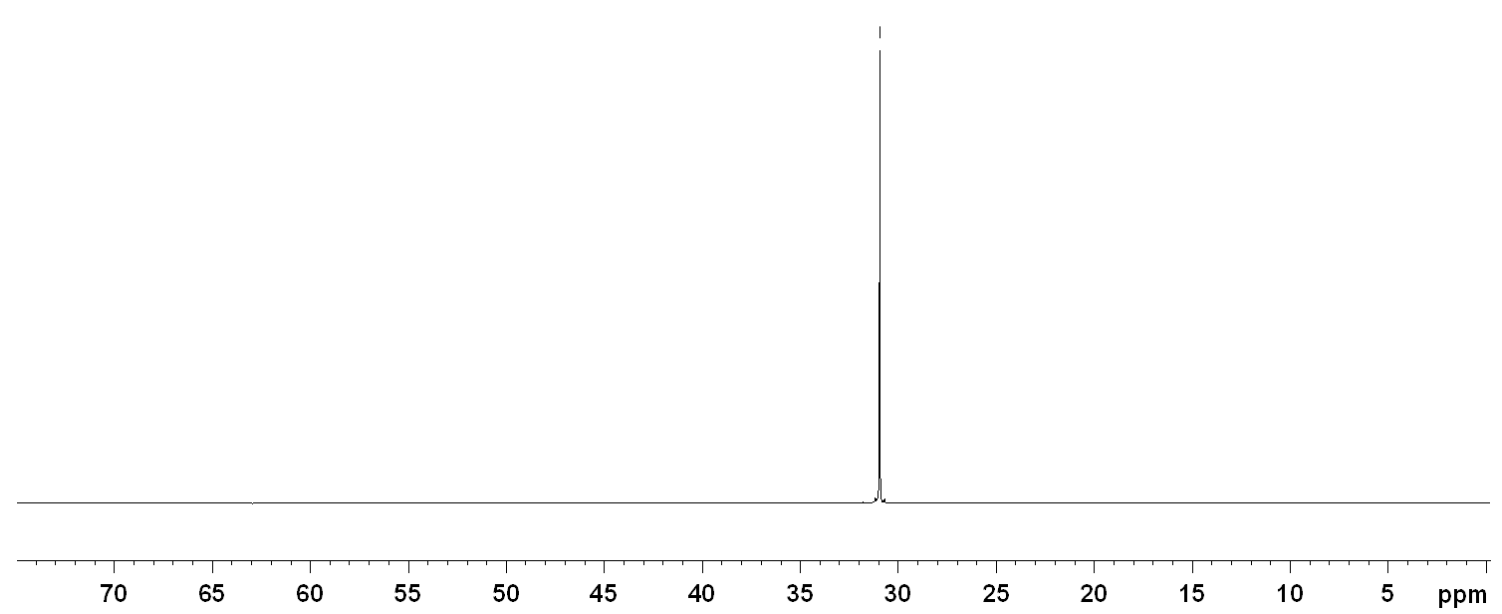


$(R, S)$-Dimethyl 2-(tert-butilsulfinamide)-2-(2'-naphthyl)ethylfosfonate, $18(R, S)$.

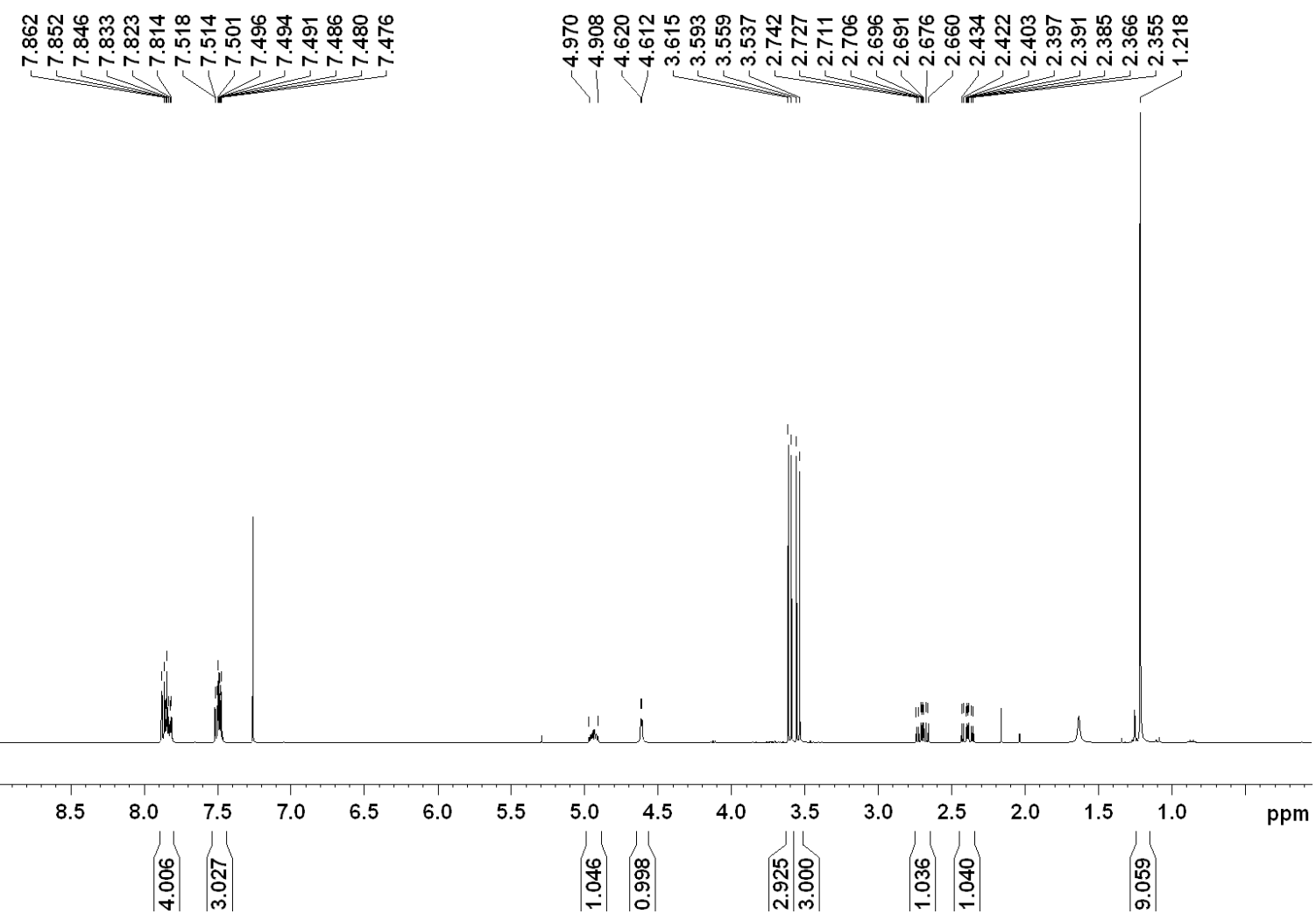

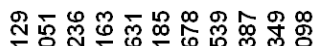

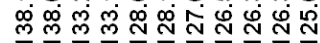

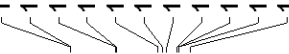

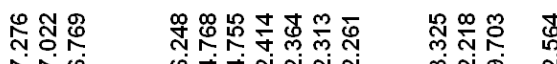

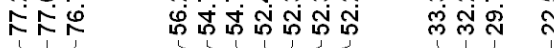

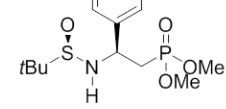

${ }^{13} \mathrm{CNMR}\left(125 \mathrm{MHz}, \mathrm{CDCl}_{3}\right)$

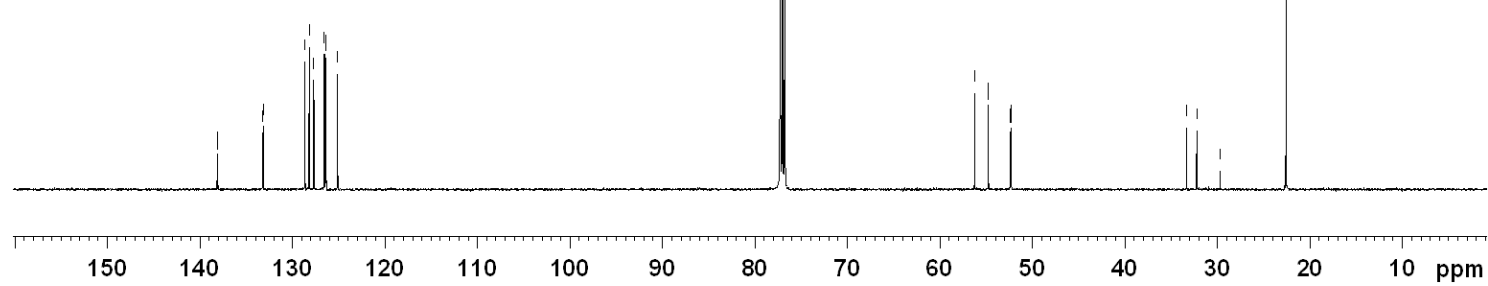




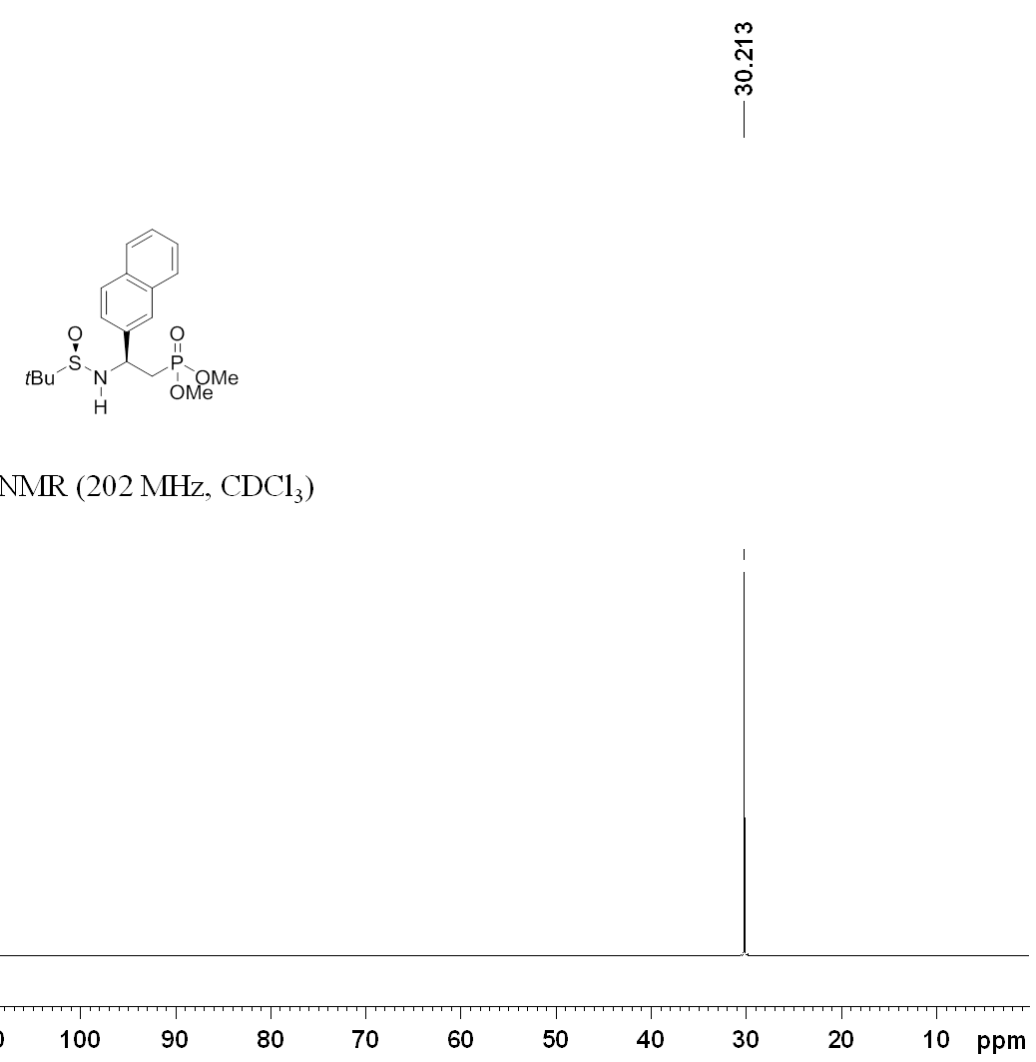

(S,R)-Methyl 2-(tert-butylsulfonamide)-2-(2'-naphthyl)ethylphenylphosphinate, 19(S,R).

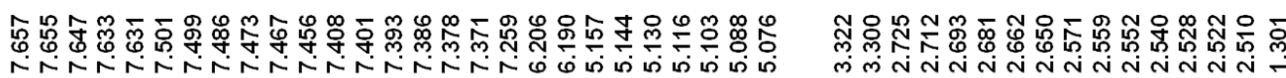

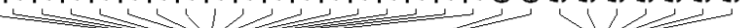

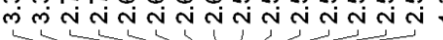

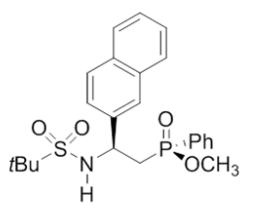

${ }^{1} \mathrm{H} \mathrm{NMR}\left(500 \mathrm{MHz}, \mathrm{CDCl}_{3}\right)$

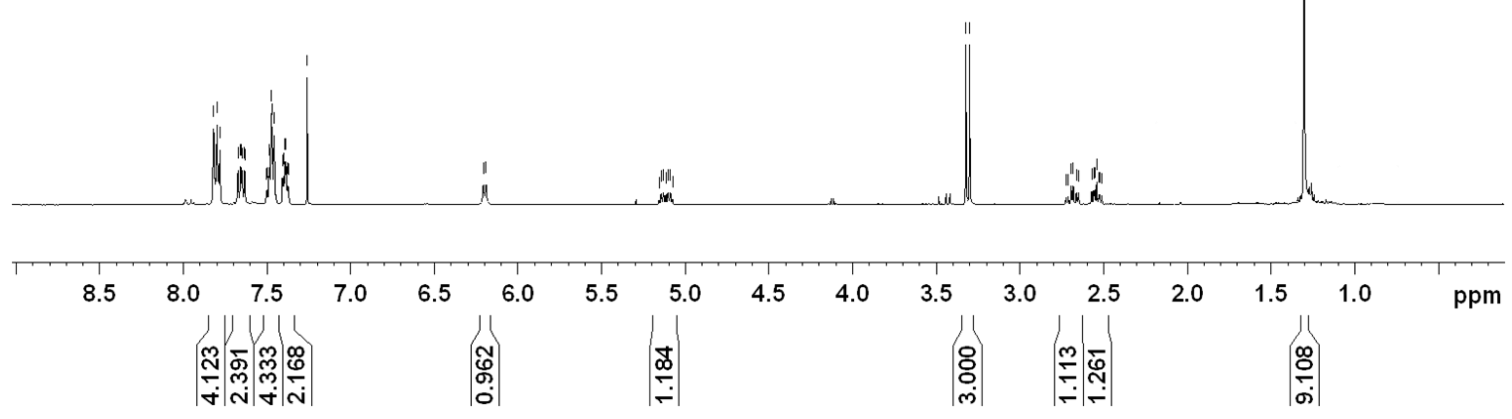




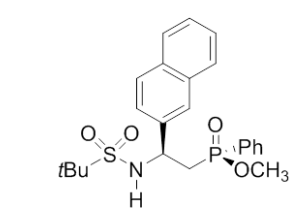

${ }^{13} \mathrm{C} \mathrm{NMR}\left(125 \mathrm{MHz}, \mathrm{CDCl}_{3}\right)$
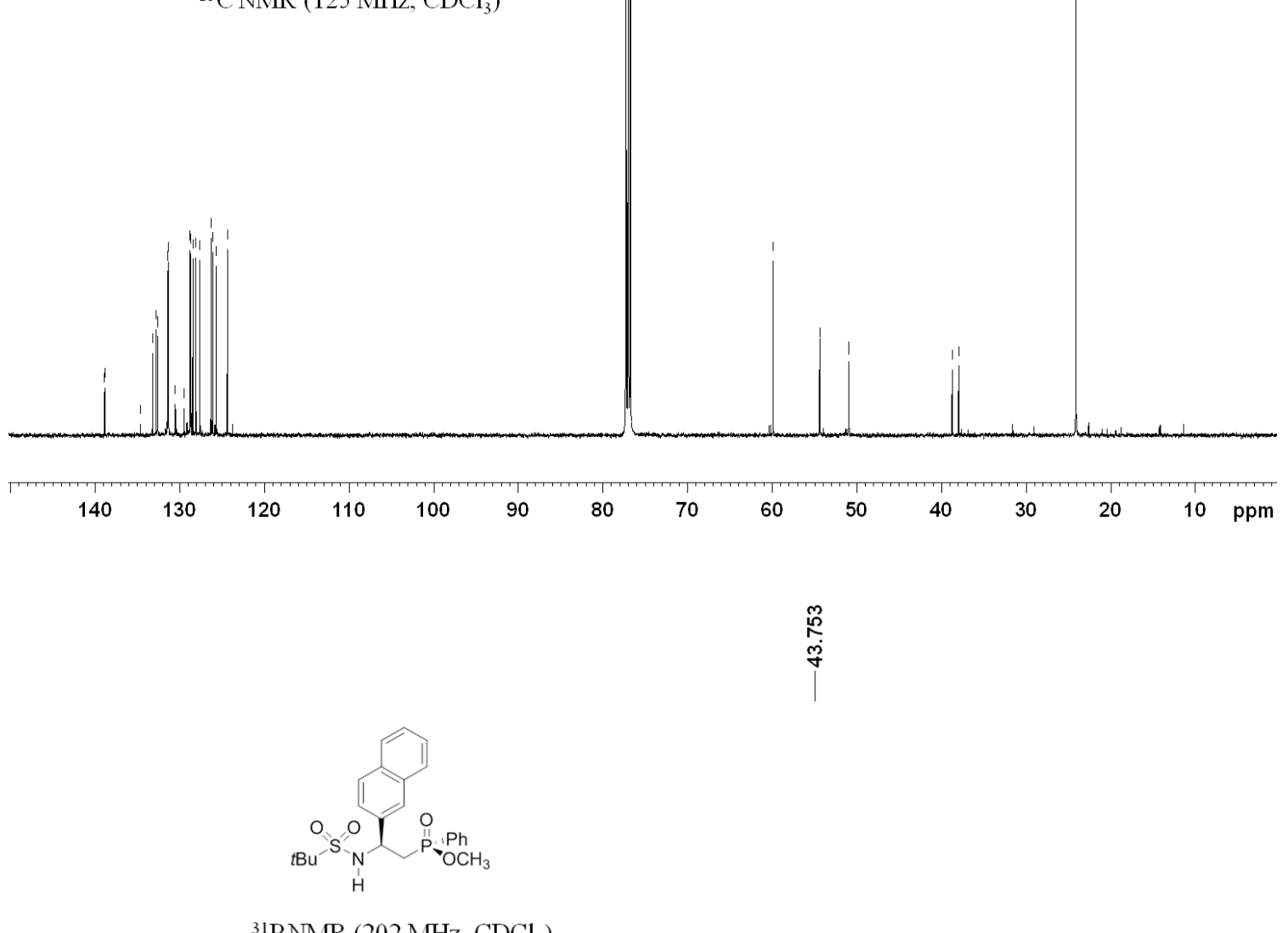

$\stackrel{m}{\stackrel{m}{\sim}}$

${ }^{31} \mathrm{PNMR}\left(202 \mathrm{MHz}, \mathrm{CDCl}_{3}\right)$

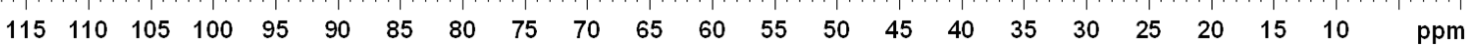




\section{N-(1-Phenylethylidene)aniline, 13.}

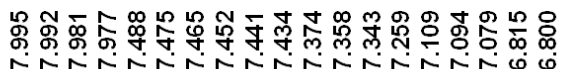

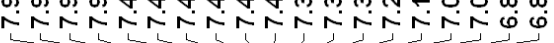

$$
\mathrm{Ph} \stackrel{\mathrm{N}}{\mathrm{N}}_{\mathrm{Me}}^{\mathrm{Ph}}
$$

${ }^{1} \mathrm{H} \mathrm{NMR}\left(500 \mathrm{MHz}, \mathrm{CDCl}_{3}\right)$

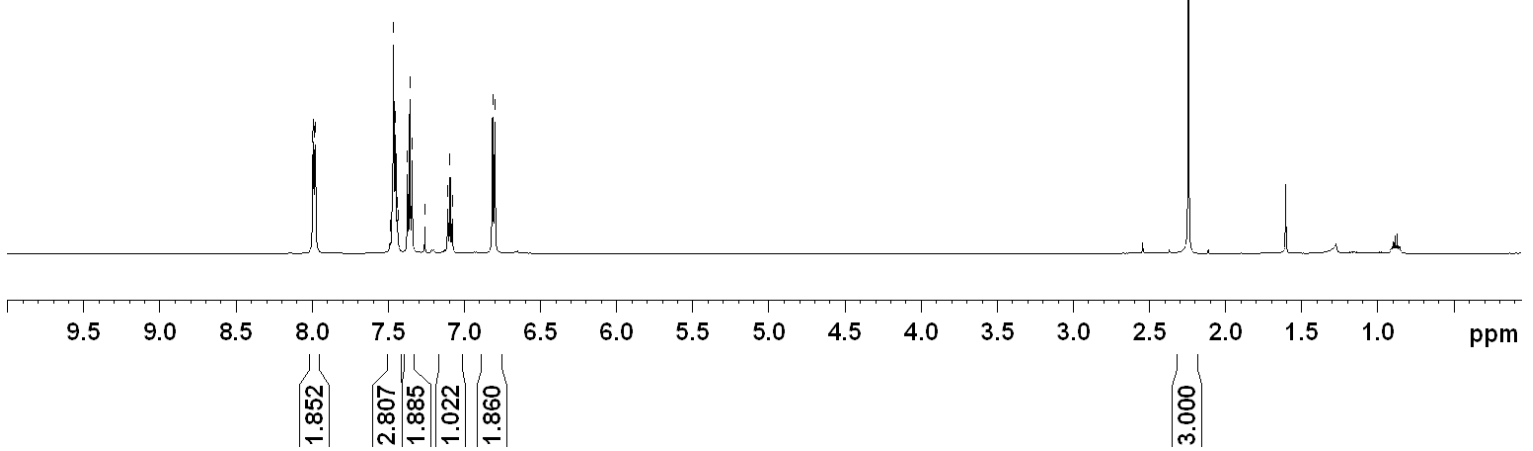

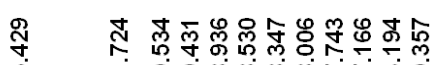

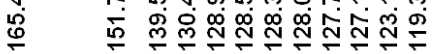

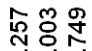

र́ri

$Y$

尊

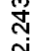

$$
\mathrm{Ph}_{\mathrm{Ph}}^{\mathrm{N}_{\mathrm{Nh}}^{\mathrm{Ph}}}
$$

${ }^{13} \mathrm{C} \mathrm{NMR}\left(125 \mathrm{MHz}, \mathrm{CDCl}_{3}\right)$

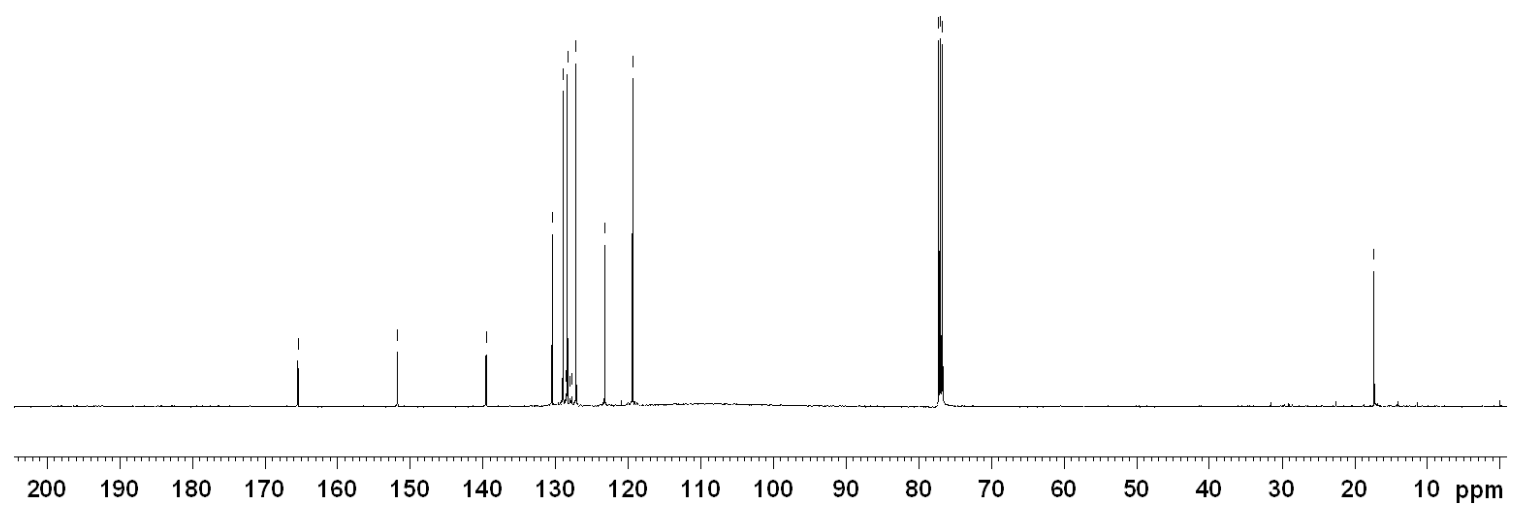




\section{$N$-(1-Phenylethylidene)-p-methoxyaniline, 20.}

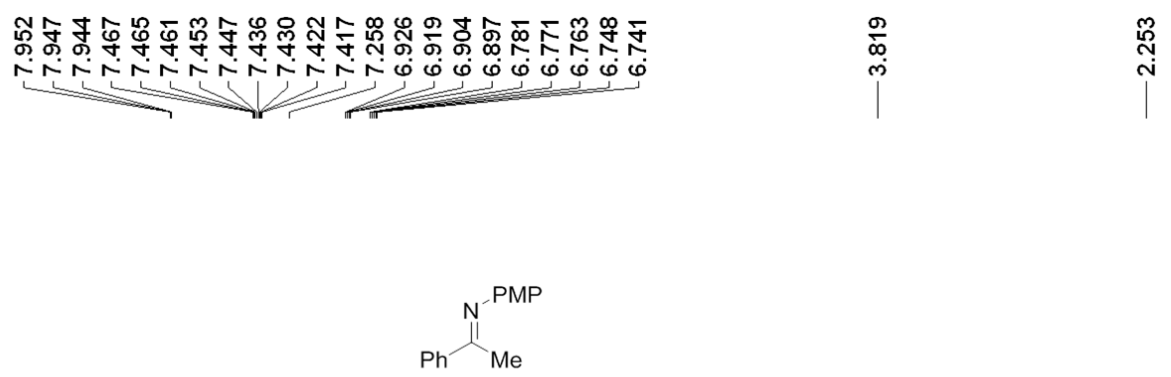

${ }^{1} \mathrm{H} \mathrm{NMR}\left(300 \mathrm{MHz}, \mathrm{CDCl}_{3}\right)$
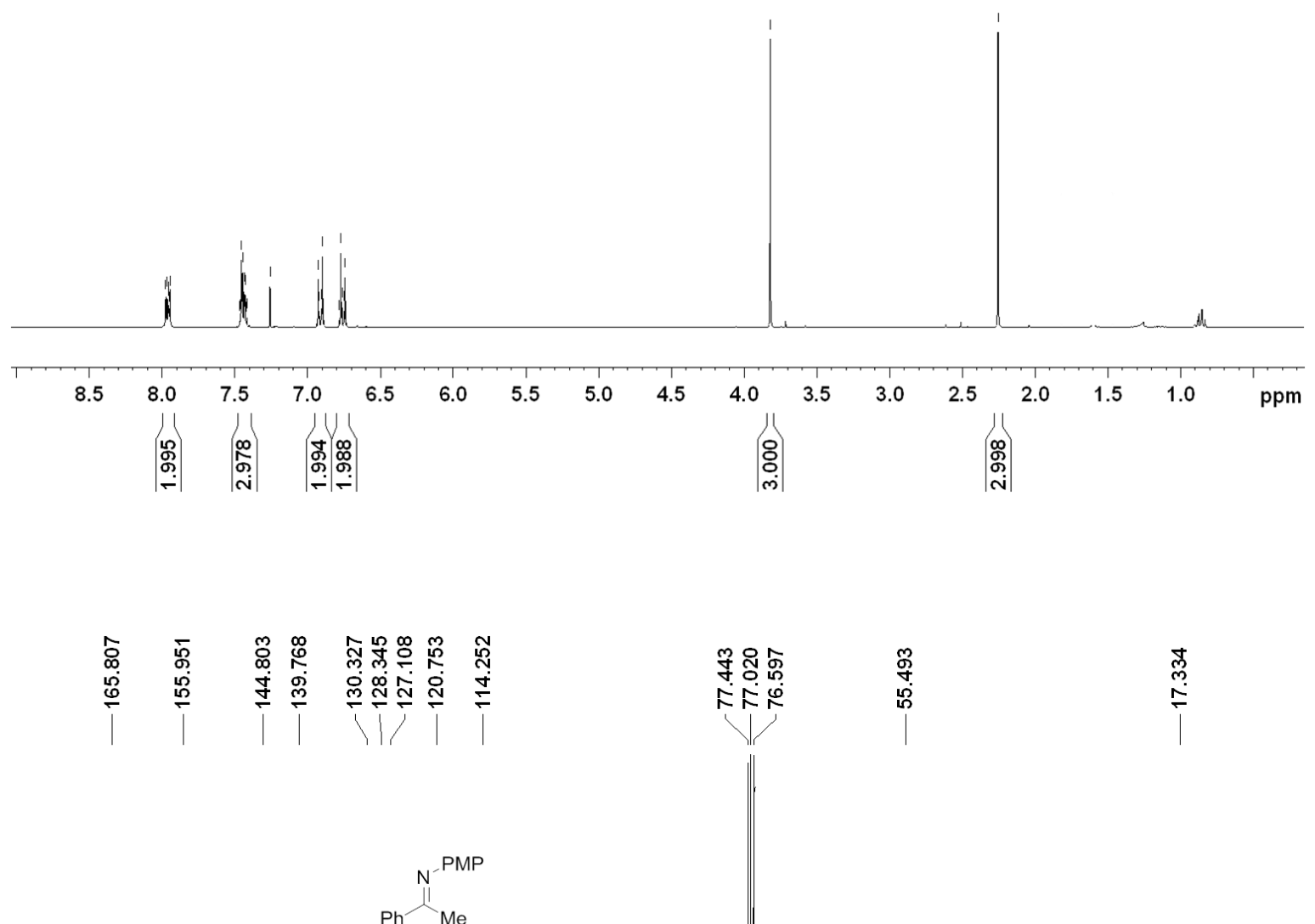

${ }^{13} \mathrm{C} \mathrm{NMR}\left(75 \mathrm{MHz}, \mathrm{CDCl}_{3}\right)$

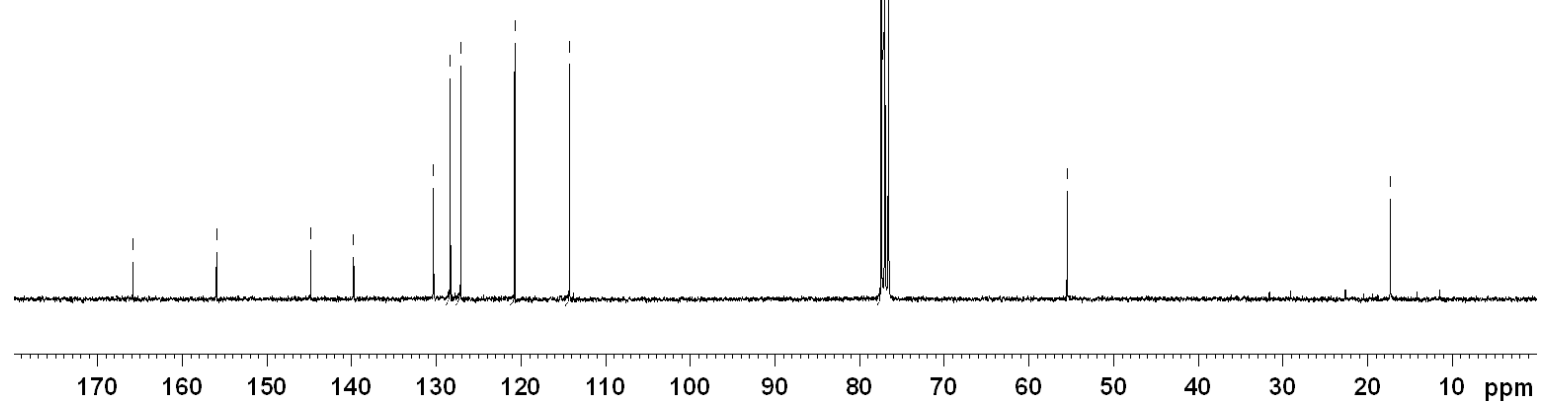




\section{N-1-(p-Methoxyphenyl)ethylidene]aniline, 21.}
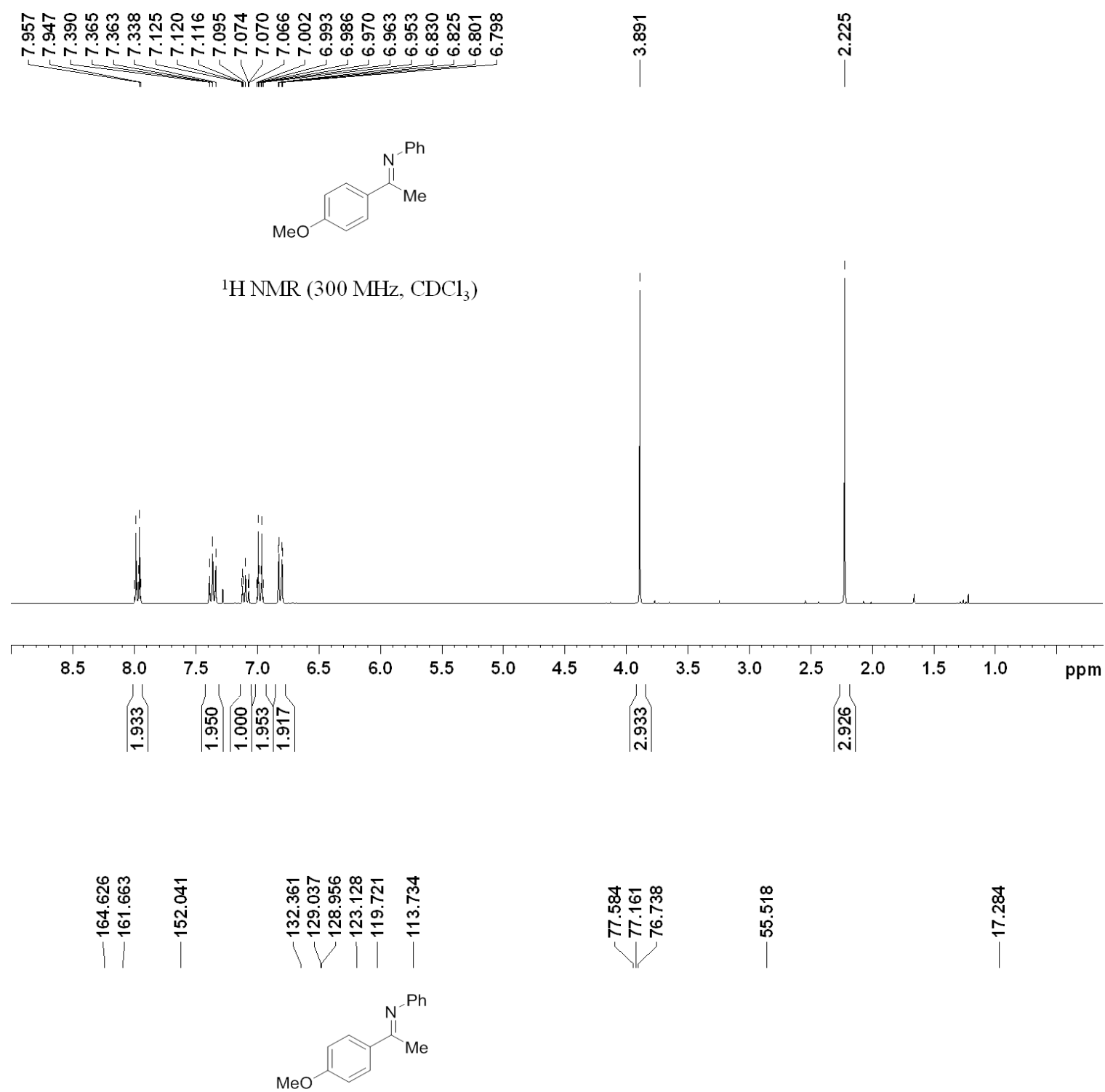

落市点

${ }^{13} \mathrm{CNMR}\left(75 \mathrm{MHz}, \mathrm{CDCl}_{3}\right)$

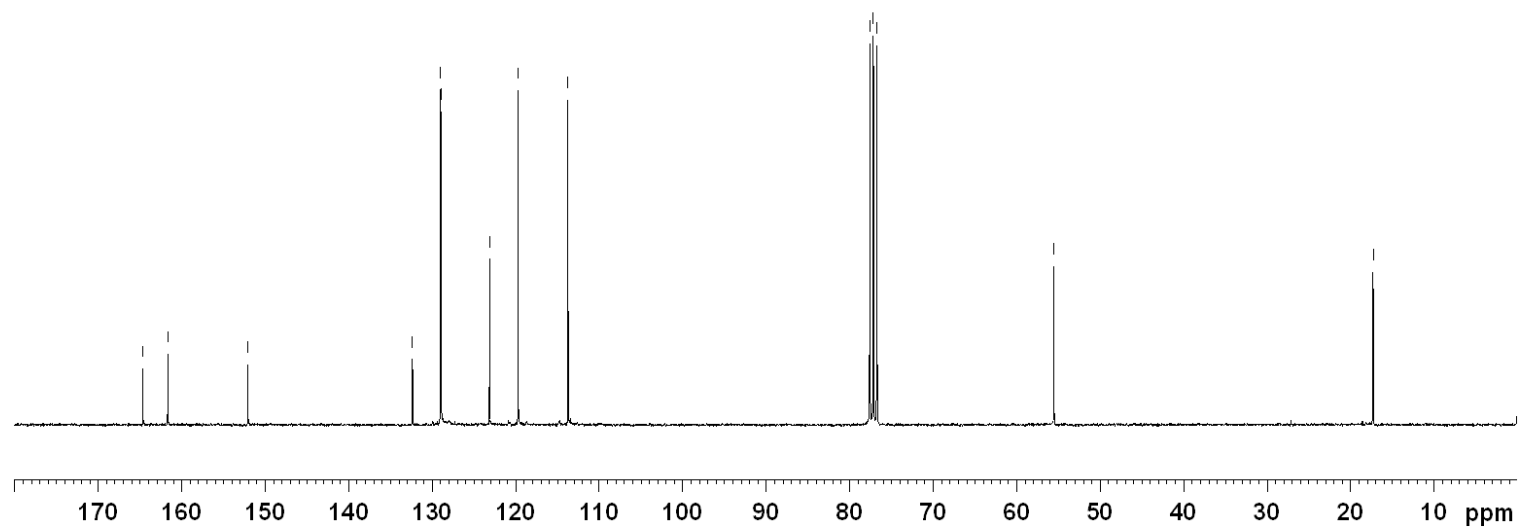


$N$-[1-(p-Methoxyphenyl)ethylidene]-p-methoxyaniline, 22.

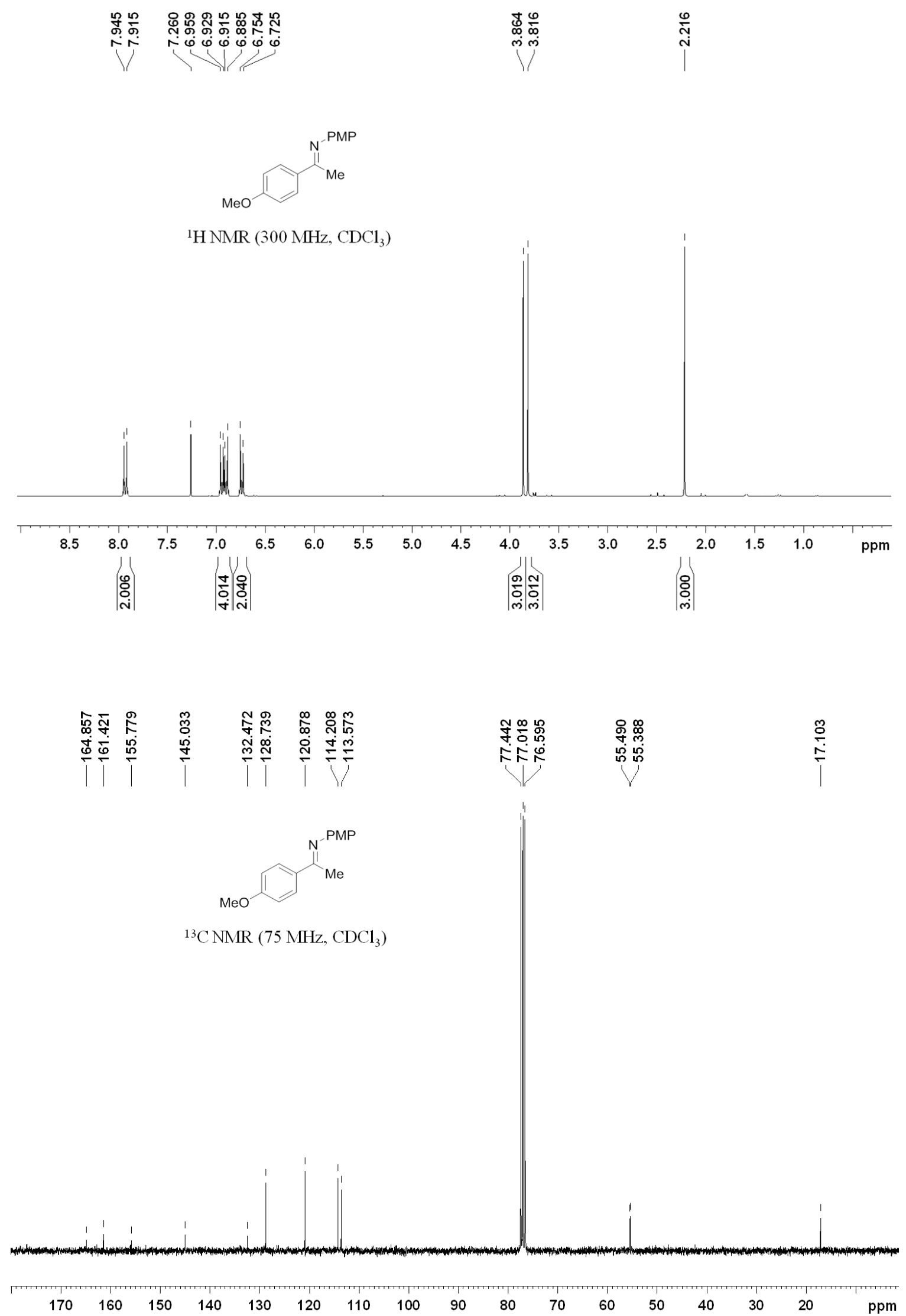




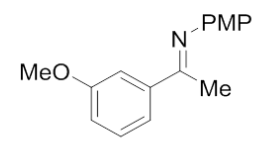

${ }^{1} \mathrm{HNMR}\left(300 \mathrm{MHz}, \mathrm{CDCl}_{3}\right)$

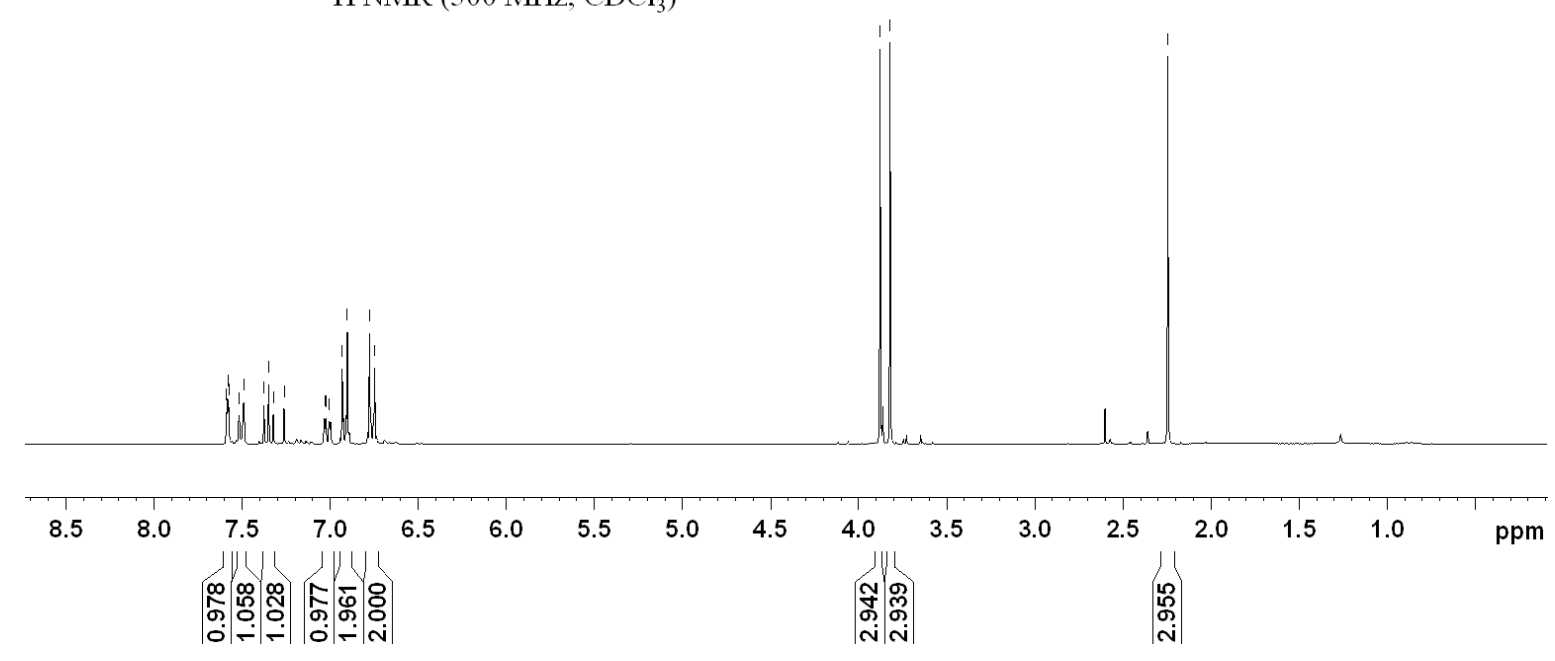

N-[1-(2'-Bromophenyl)ethylidene]aniline, 24.

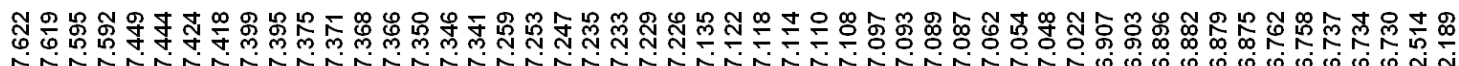

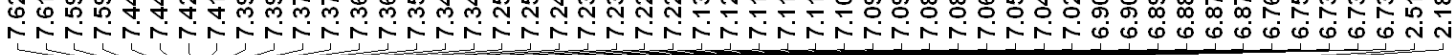

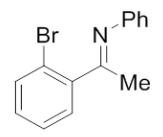

${ }^{1} \mathrm{H} \mathrm{NMR}\left(300 \mathrm{MHz}, \mathrm{CDCl}_{3}\right)$

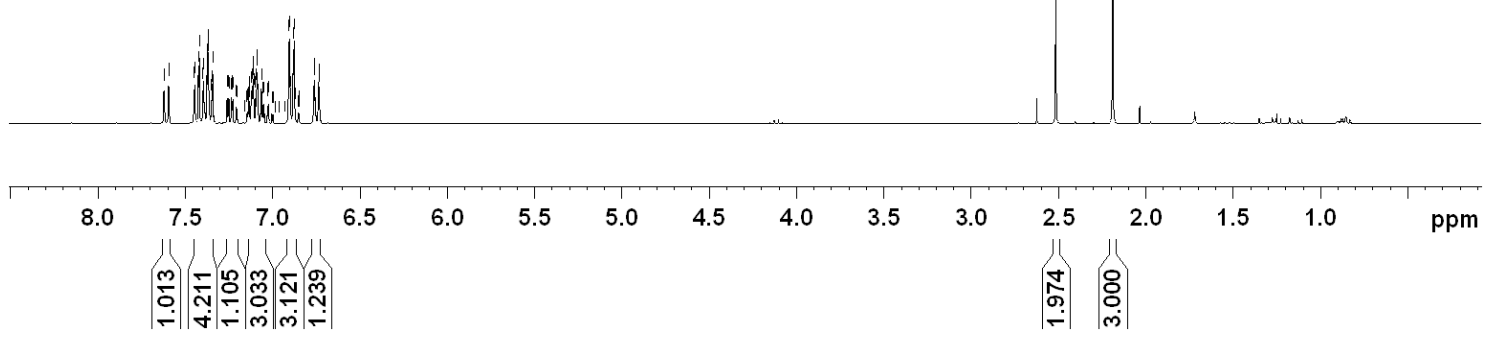




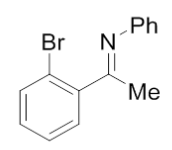

${ }^{13} \mathrm{CNMR}\left(75 \mathrm{MHz}, \mathrm{CDCl}_{3}\right)$

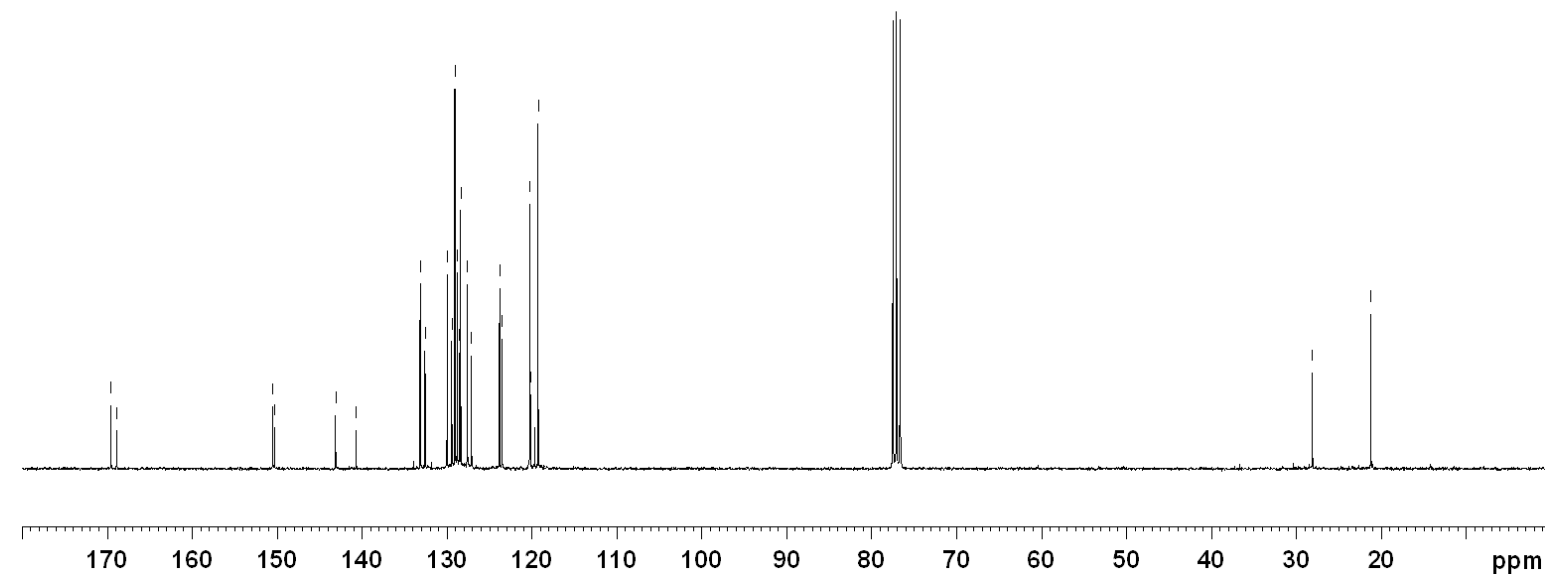

\section{$N$-[1-(3'-Bromophenyl)ethylidene]-p-methoxyaniline, 25.}

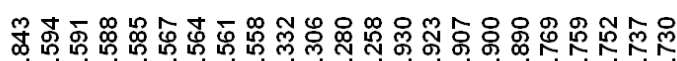

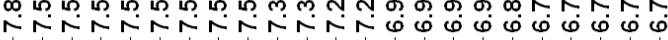
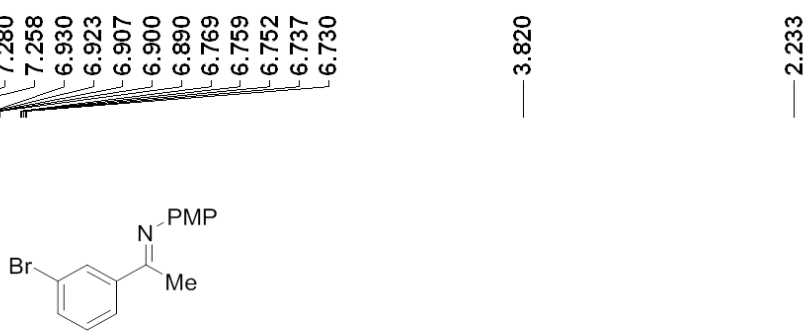

${ }^{1} \mathrm{H} \mathrm{NMR}\left(300 \mathrm{MHz}, \mathrm{CDCl}_{3}\right)$

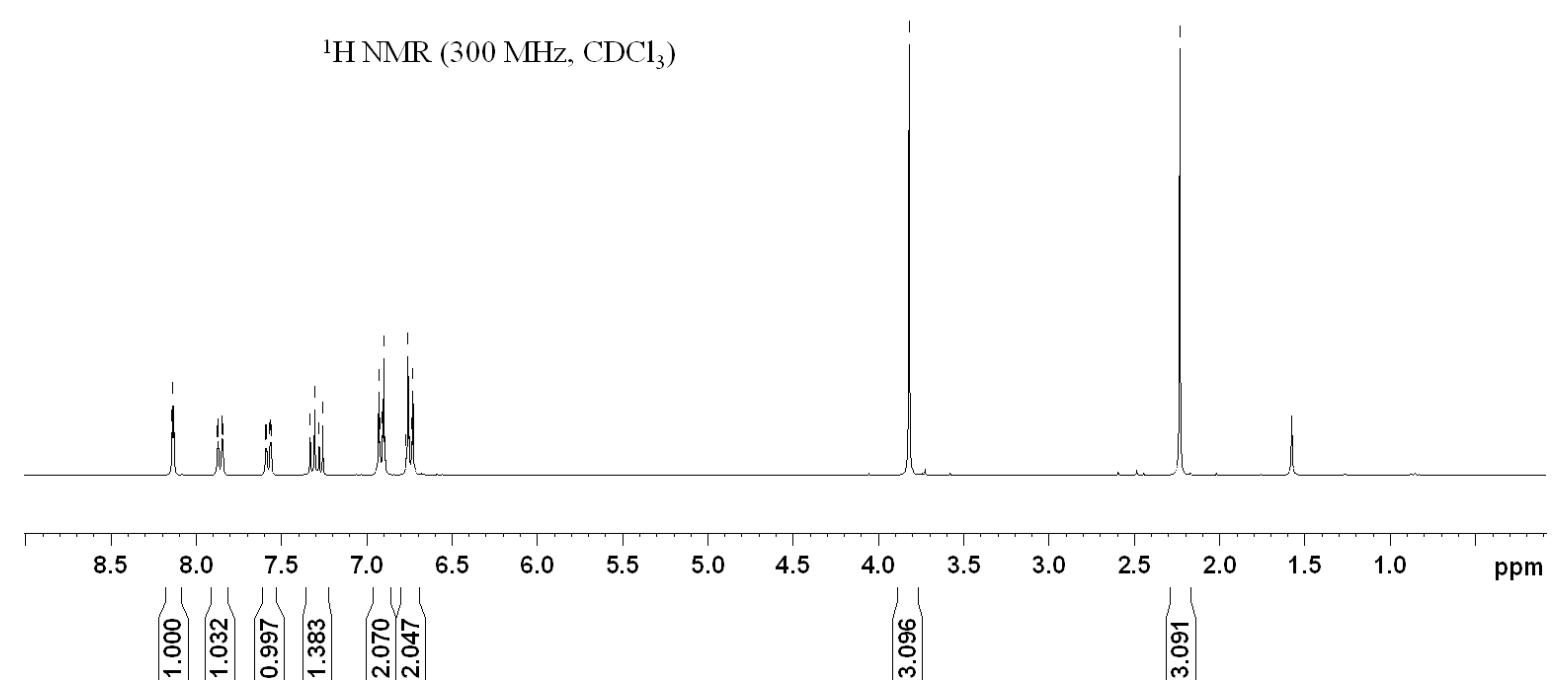



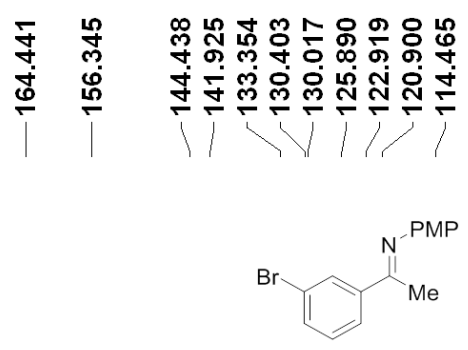

${ }^{13} \mathrm{CNMR}\left(75 \mathrm{MHz}, \mathrm{CDCl}_{3}\right)$

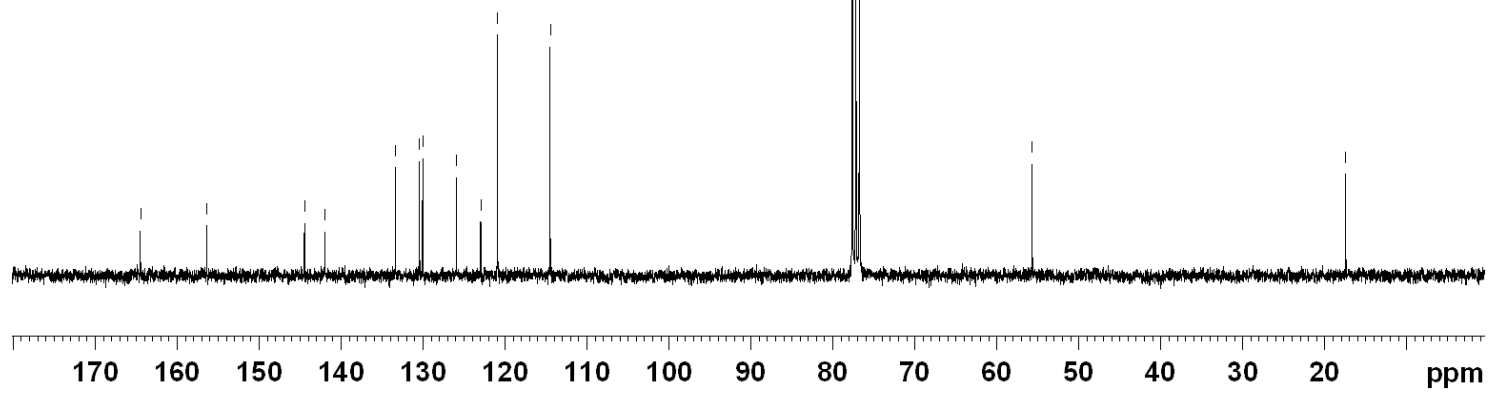

$N$-[1-(4'-Bromophenyl)ethylidene]-p-methoxyaniline, 26.

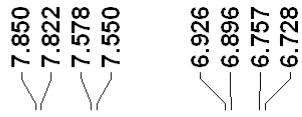
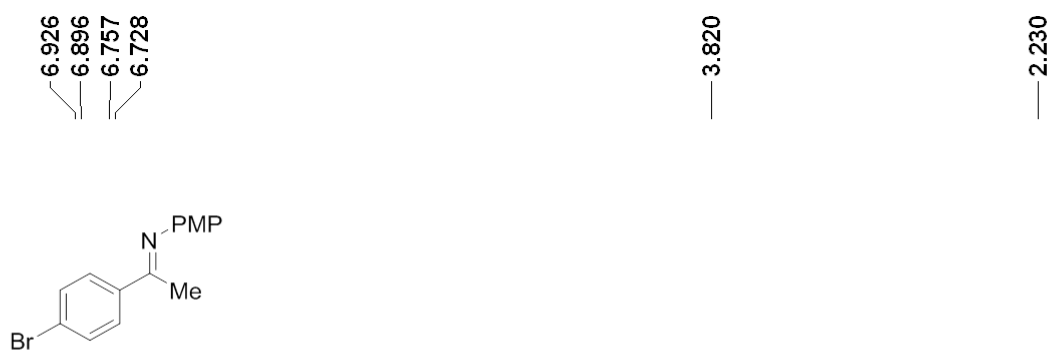

${ }^{1} \mathrm{H} \mathrm{NMR}\left(300 \mathrm{MHz}, \mathrm{CDCl}_{3}\right)$

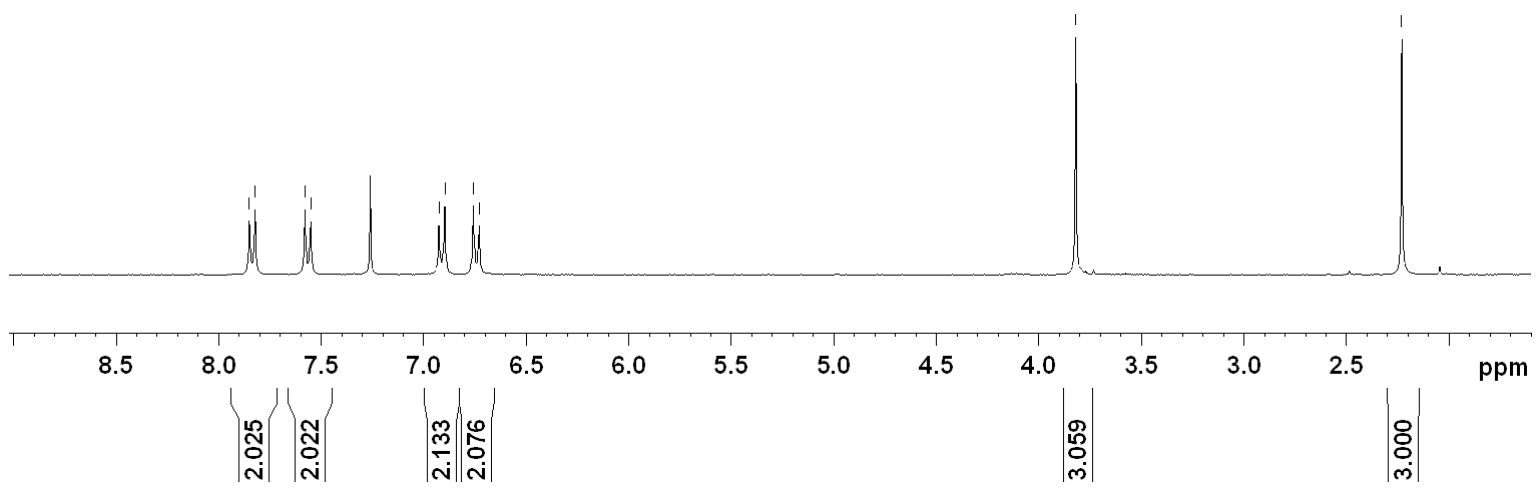


$N$-[1-(p-Nitrophenyl)ethylidene]-p-methoxyaniline, 27.

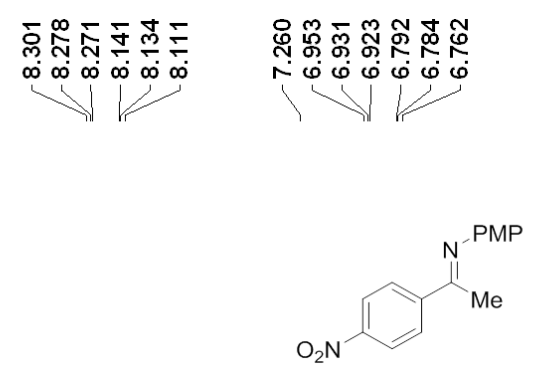

${ }^{1} \mathrm{H} N M R\left(300 \mathrm{MHz}, \mathrm{CDCl}_{3}\right)$
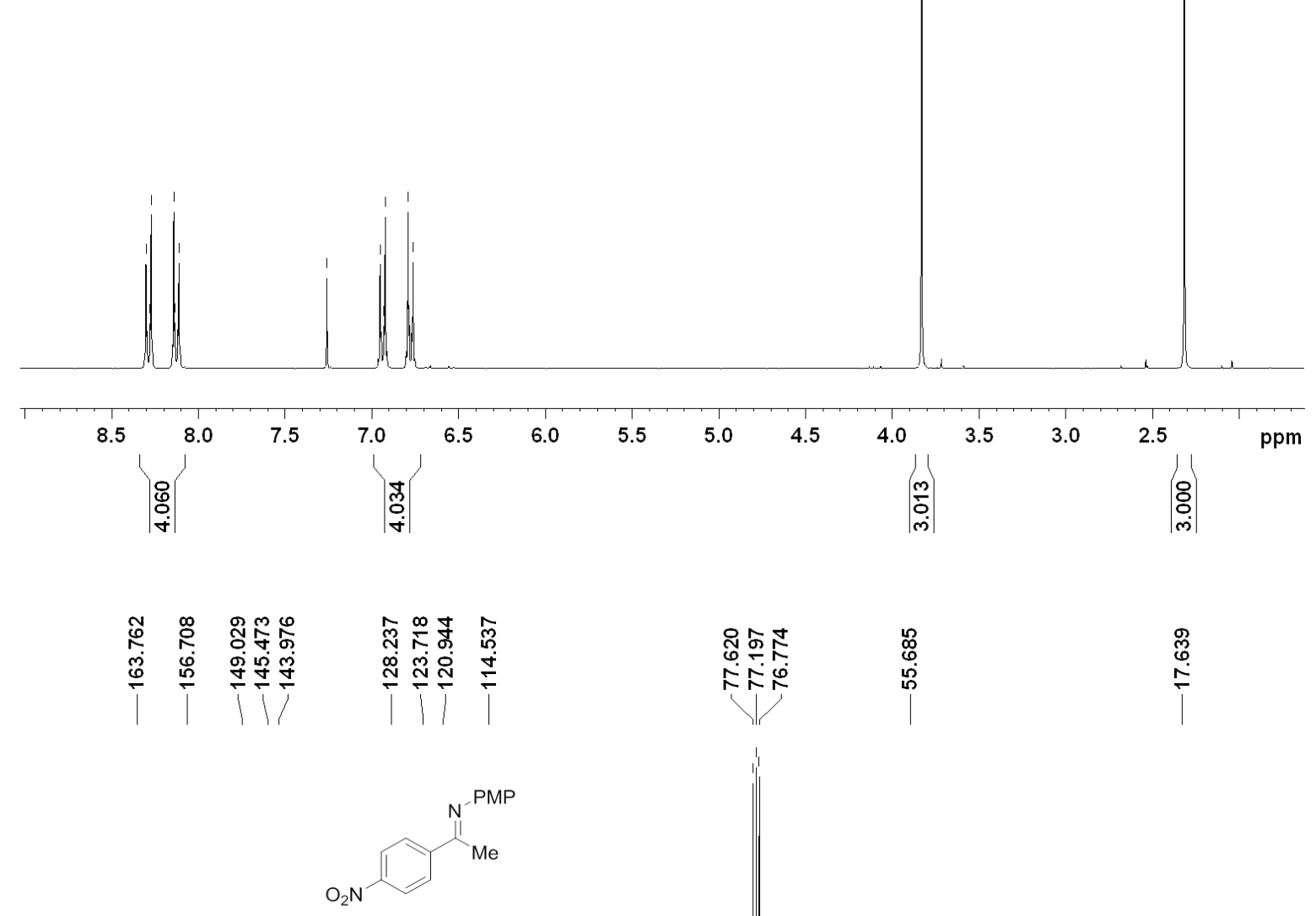

${ }^{13} \mathrm{CNMR}\left(75 \mathrm{MHz}, \mathrm{CDCl}_{3}\right)$

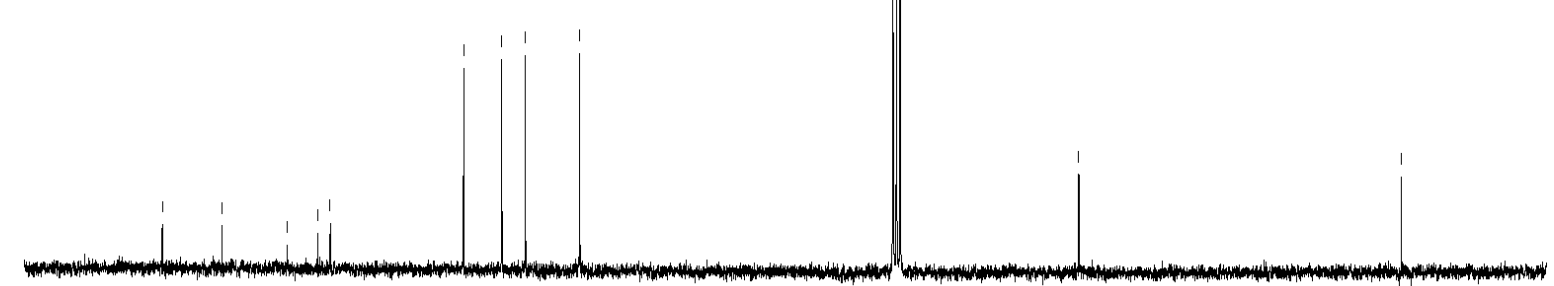

$\begin{array}{lllllllllllllllll}170 & 160 & 150 & 140 & 130 & 120 & 110 & 100 & 90 & 80 & 70 & 60 & 50 & 40 & 30 & 20 & 10\end{array}$ 


\section{$N$-[1-(2'-Naphthyl)ethylidene]aniline, 28.}

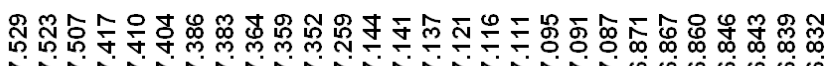

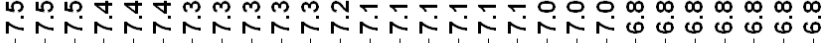

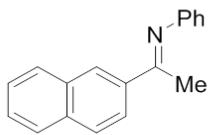

${ }^{1} \mathrm{H} \mathrm{NMR}\left(300 \mathrm{MHz}, \mathrm{CDCl}_{3}\right)$
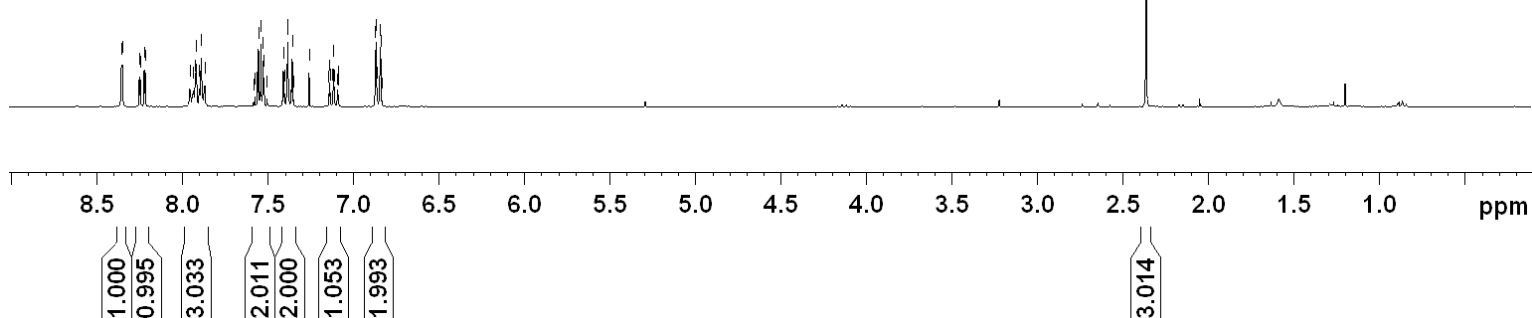

$N$-[1-(2'-Naphthyl)ethylidene]-p-methoxyaniline, 29.

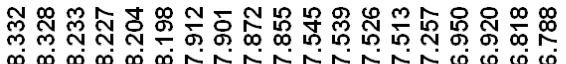

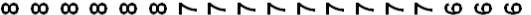

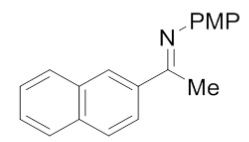

${ }^{1} \mathrm{H} \mathrm{NMR}\left(300 \mathrm{MHz}, \mathrm{CDCl}_{3}\right)$

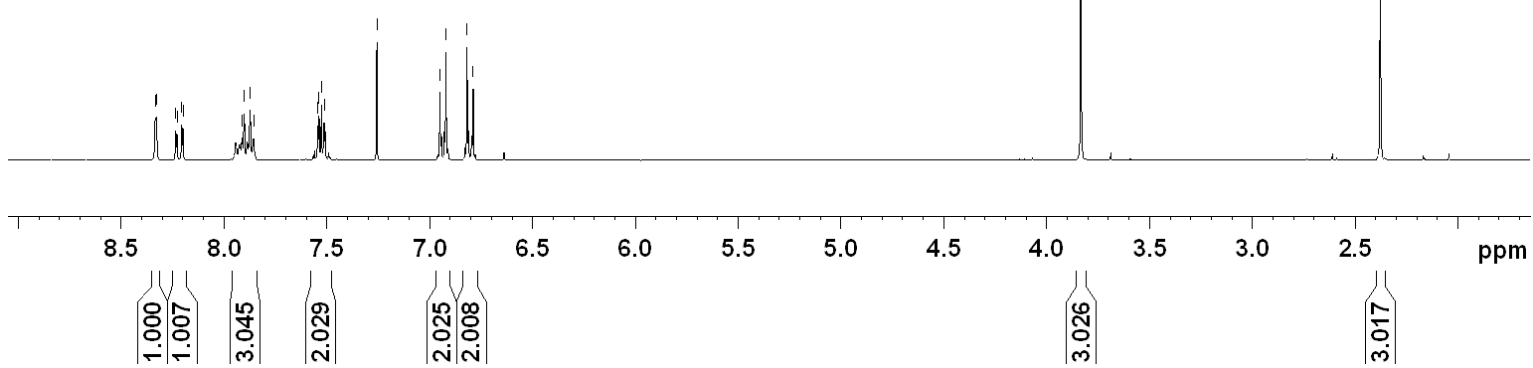



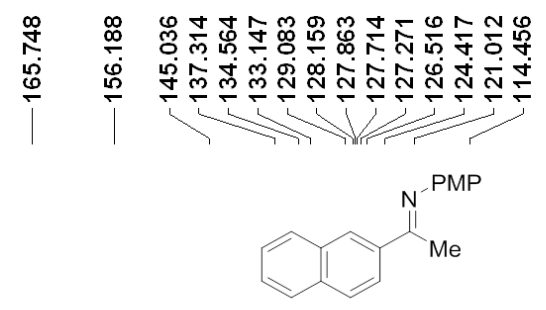

${ }^{13} \mathrm{C} \mathrm{NMR}\left(75 \mathrm{MHz}, \mathrm{CDCl}_{3}\right)$

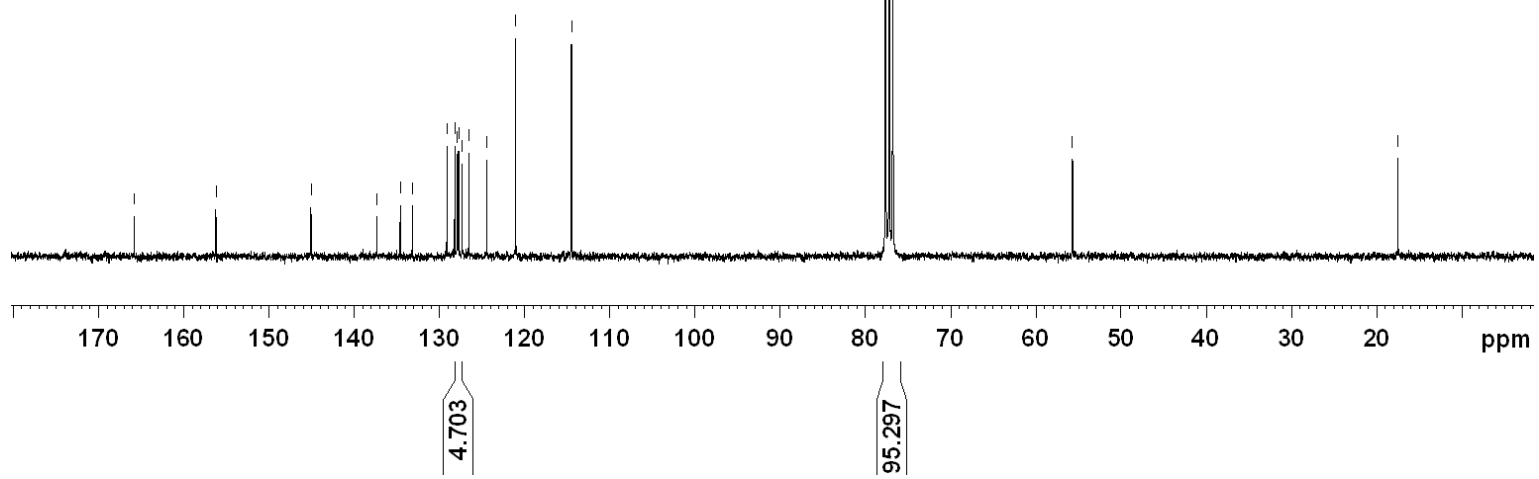

(R)- $N$-(1-Phenyletil)aniline, $14 R$.

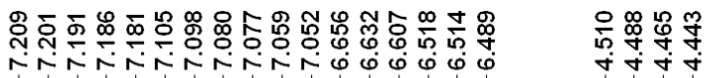

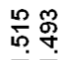

$$
\mathrm{Ph}_{\mathrm{He}}^{\mathrm{HN}^{-\mathrm{Ph}}} \mathrm{Me}
$$

${ }^{1} \mathrm{H} \mathrm{NMR}\left(300 \mathrm{MHz}, \mathrm{CDCl}_{3}\right)$

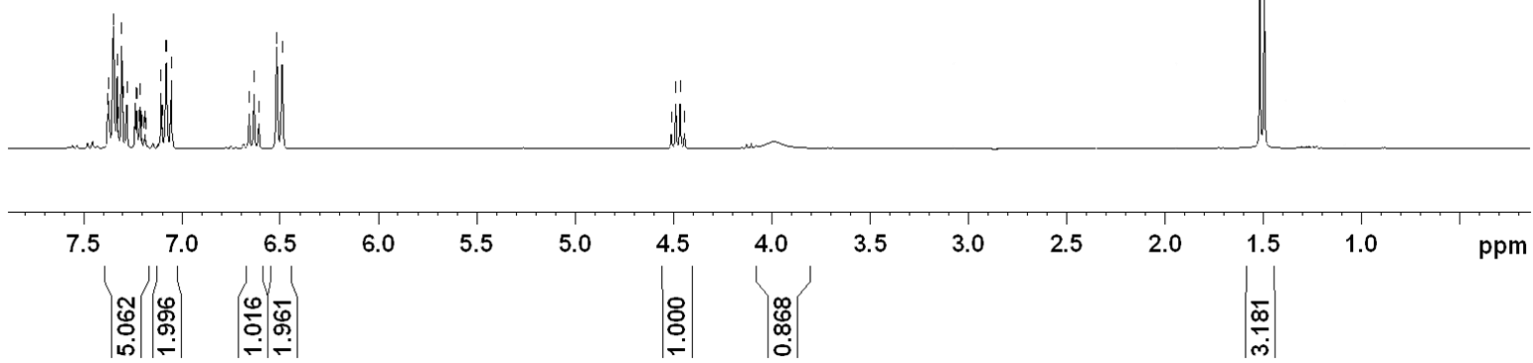


(R)-N-[(1-Phenyl)ethyl]-p-methoxyaniline, $30 R$.

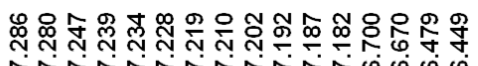

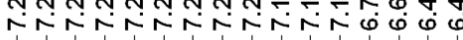

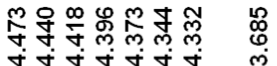

英 品

$1 \ldots$

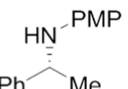

$\mathrm{Ph} \mathrm{Me}$

${ }^{1} \mathrm{H} \mathrm{NMR}\left(300 \mathrm{MHz}, \mathrm{CDCl}_{3}\right)$

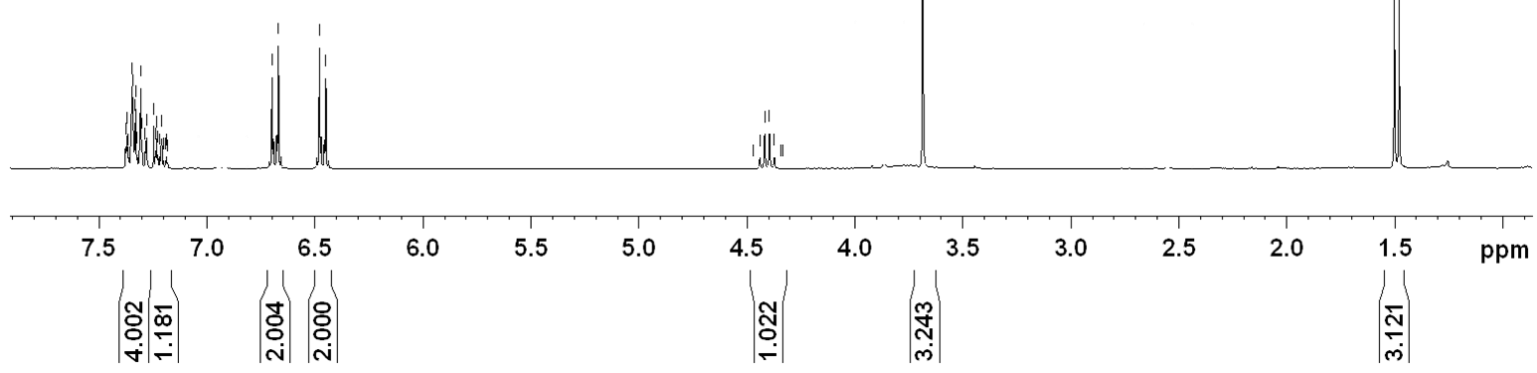

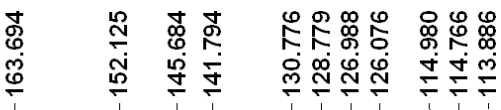

号 ஸ்ุ

トR்

柋㸃

$\stackrel{\leftrightarrow}{\stackrel{n}{*}}$

Y

ผ

$$
\mathrm{Ph} \stackrel{\mathrm{MN}}{\mathrm{H}} \stackrel{\mathrm{PMP}}{\mathrm{C}}
$$

${ }^{13} \mathrm{CNMR}\left(125 \mathrm{MHz}, \mathrm{CDCl}_{3}\right)$

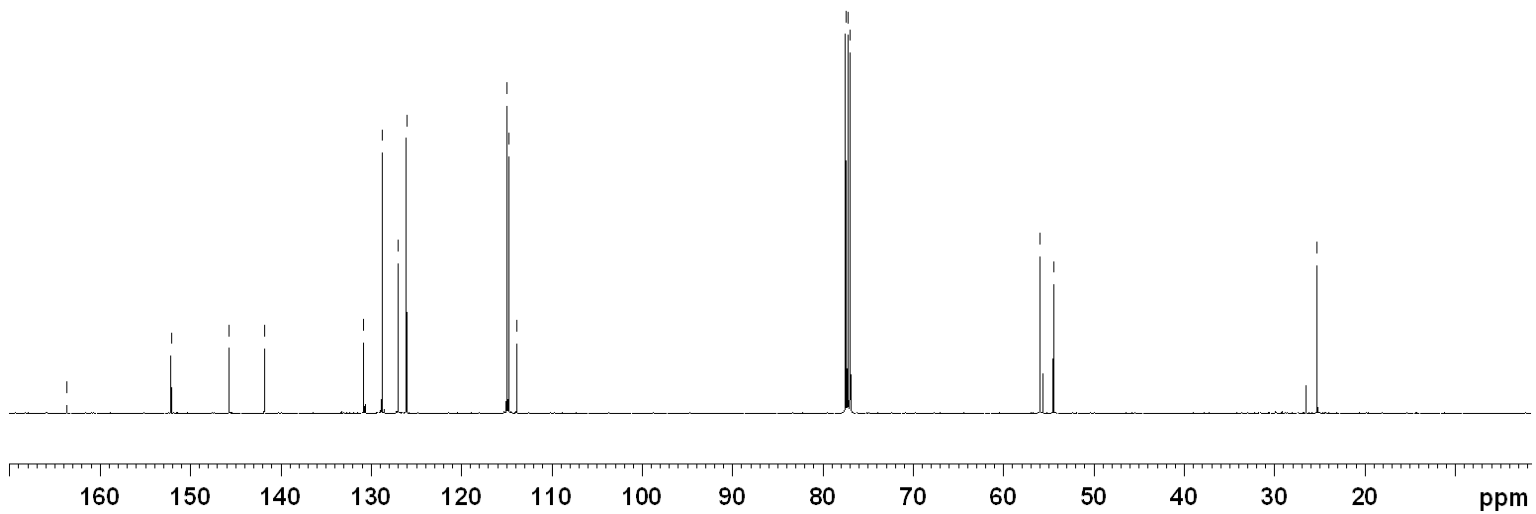


$(R)-N$-[1-(p-Methoxyphenyl)ethyl)]aniline, 31R.

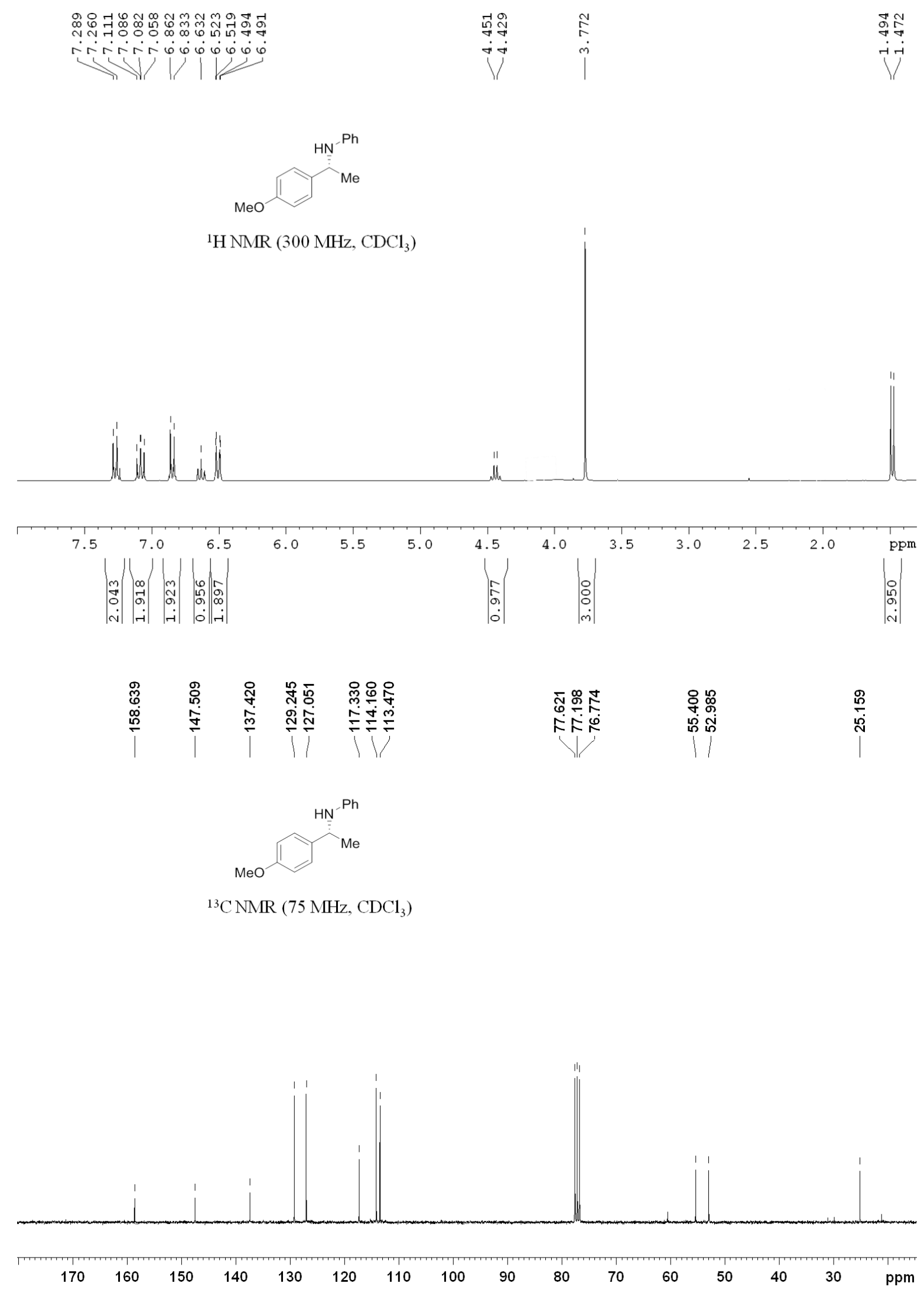


$(R)-N-[(1-(p-M e t h o x y p h e n y l) e t h y l]-p$-methoxyaniline, $32 R$.

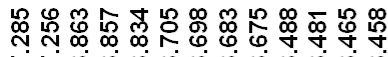

ヘN

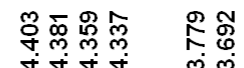

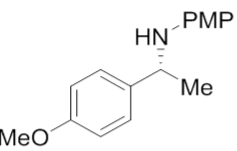

${ }^{1} \mathrm{H} \mathrm{NMR}\left(300 \mathrm{MHz}, \mathrm{CDCl}_{3}\right)$
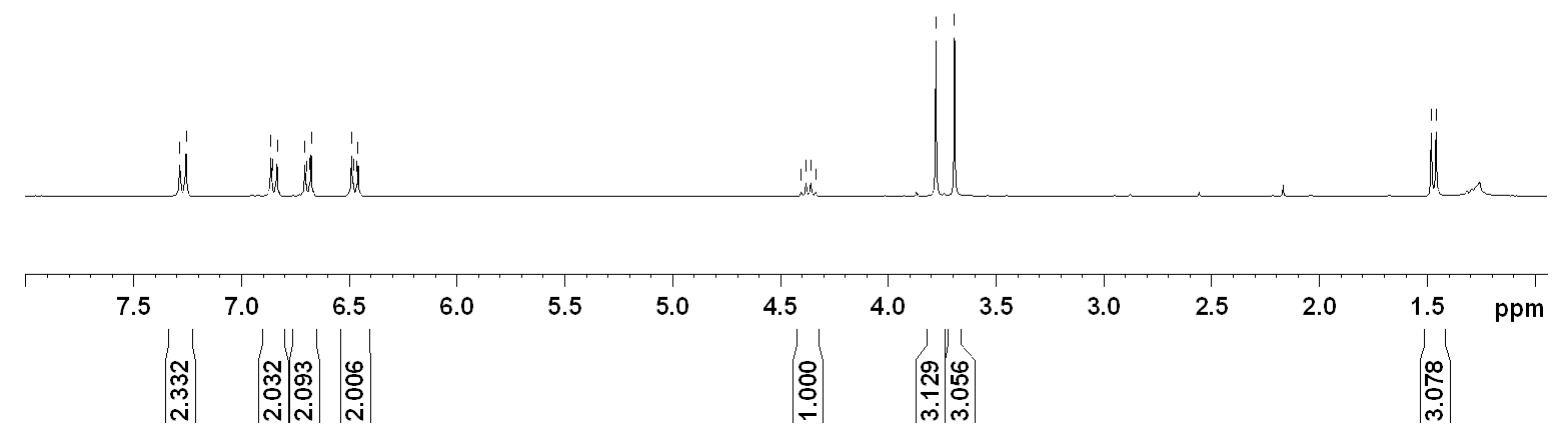

|

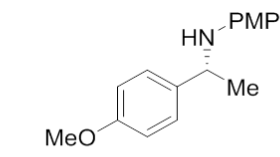

${ }^{13} \mathrm{CNMR}\left(75 \mathrm{MHz}, \mathrm{CDCl}_{3}\right)$

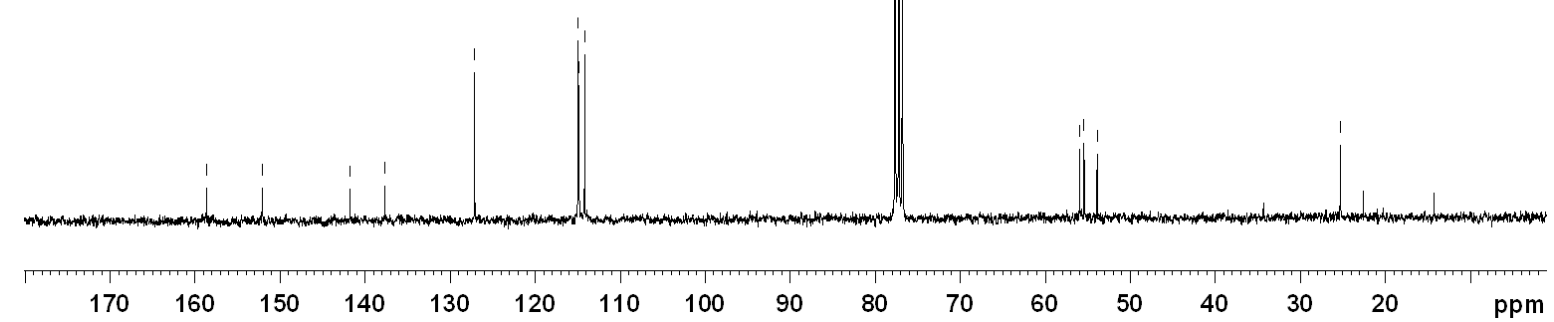


(R)-N-[(1-(3'-Methoxyphenyl)ethyl]-p-methoxyaniline, 33R.

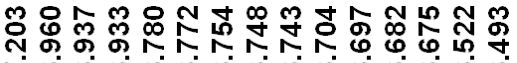
N
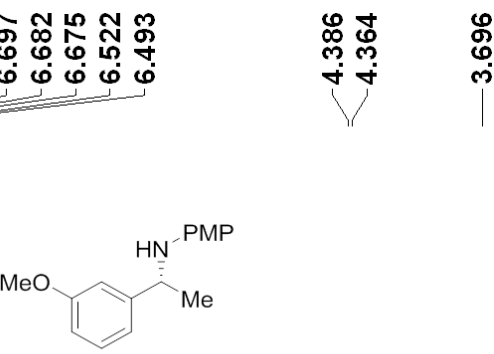

${ }^{1} \mathrm{H} \mathrm{NMR}\left(300 \mathrm{MHz}, \mathrm{CDCl}_{3}\right)$

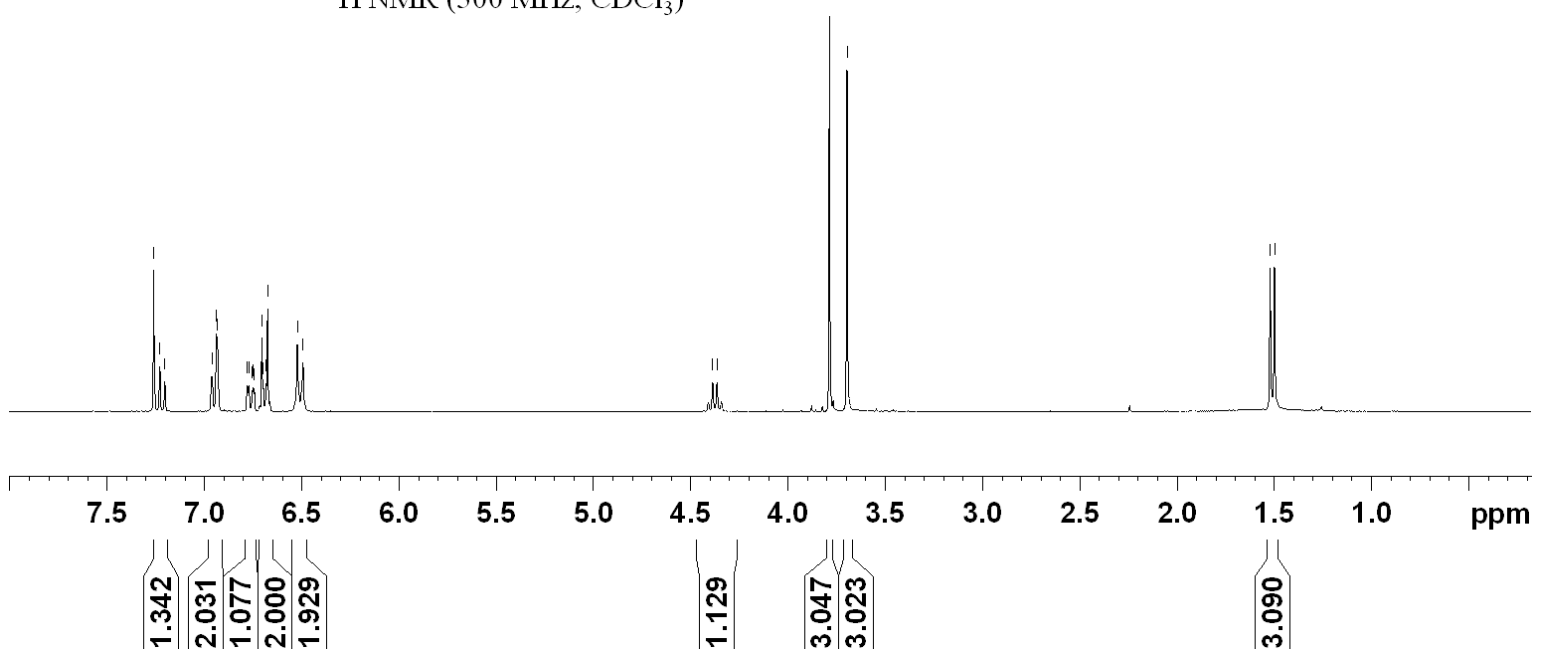

(R)-N-[(1-(2'-Bromophenyl)ethyl]aniline, 34R.

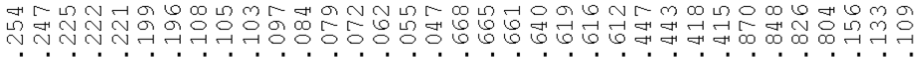

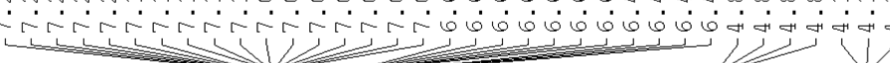

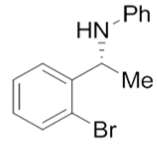

${ }^{1} \mathrm{H} \mathrm{NMR}\left(300 \mathrm{MHz}, \mathrm{CDCl}_{3}\right)$

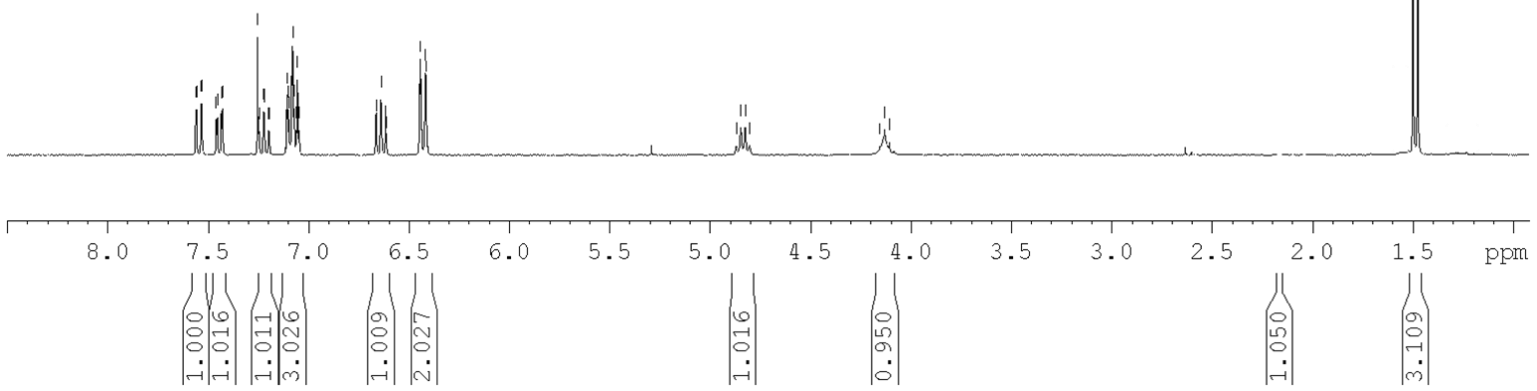


(R)- $N$-[(1-(3'-Bromophenyl)ethyl]-p-methoxyaniline, $35 R$.

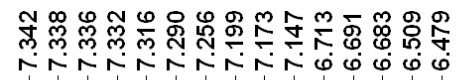

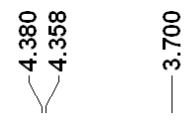

产导

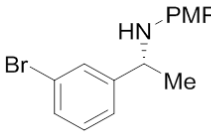

${ }^{1} \mathrm{H} \mathrm{NMR}\left(300 \mathrm{MHz}, \mathrm{CDCl}_{3}\right)$

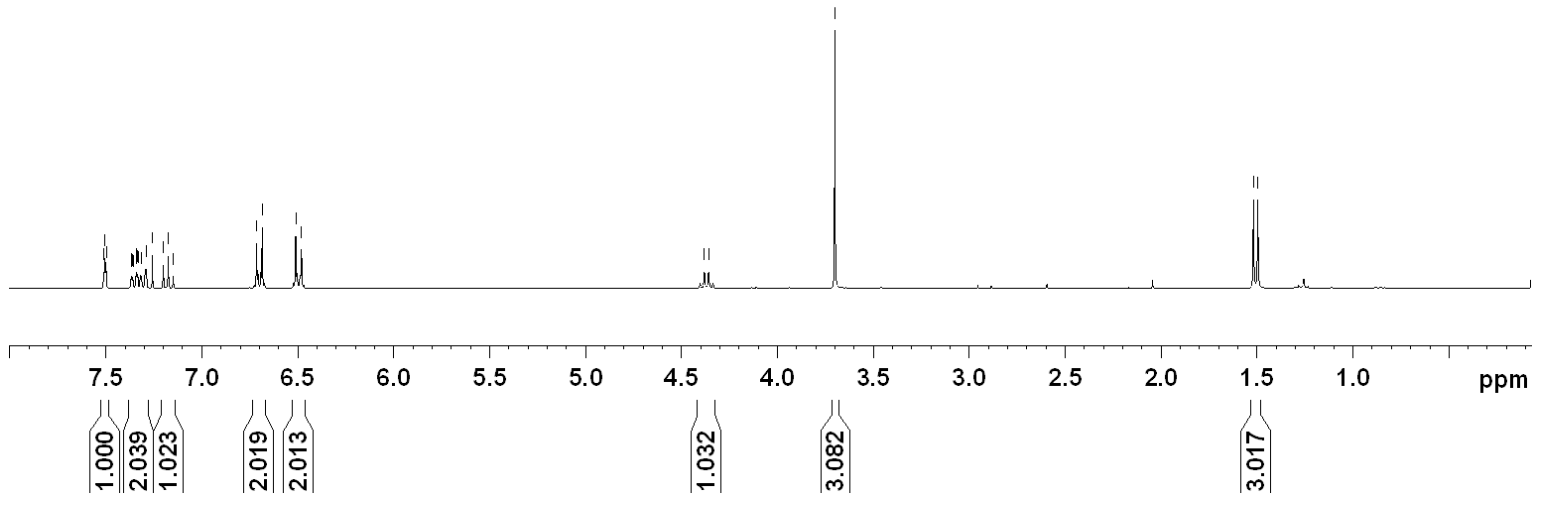

|

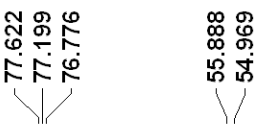

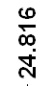

$\mathrm{Br}$

$\mathrm{HN}^{-\mathrm{PMP}}$

$\mathrm{Me}$

${ }^{13} \mathrm{CNMR}\left(75 \mathrm{MHz}, \mathrm{CDCl}_{3}\right)$

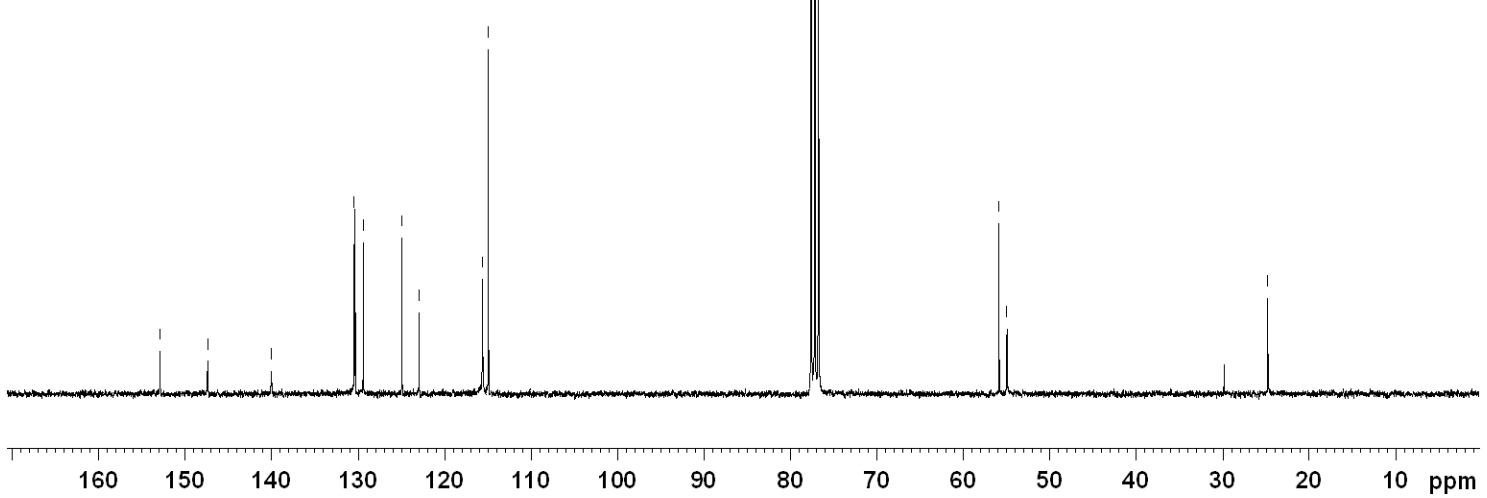


(R)-N-[(1-(p-Bromophenyl)ethyl]-p-methoxyaniline, $36 R$.

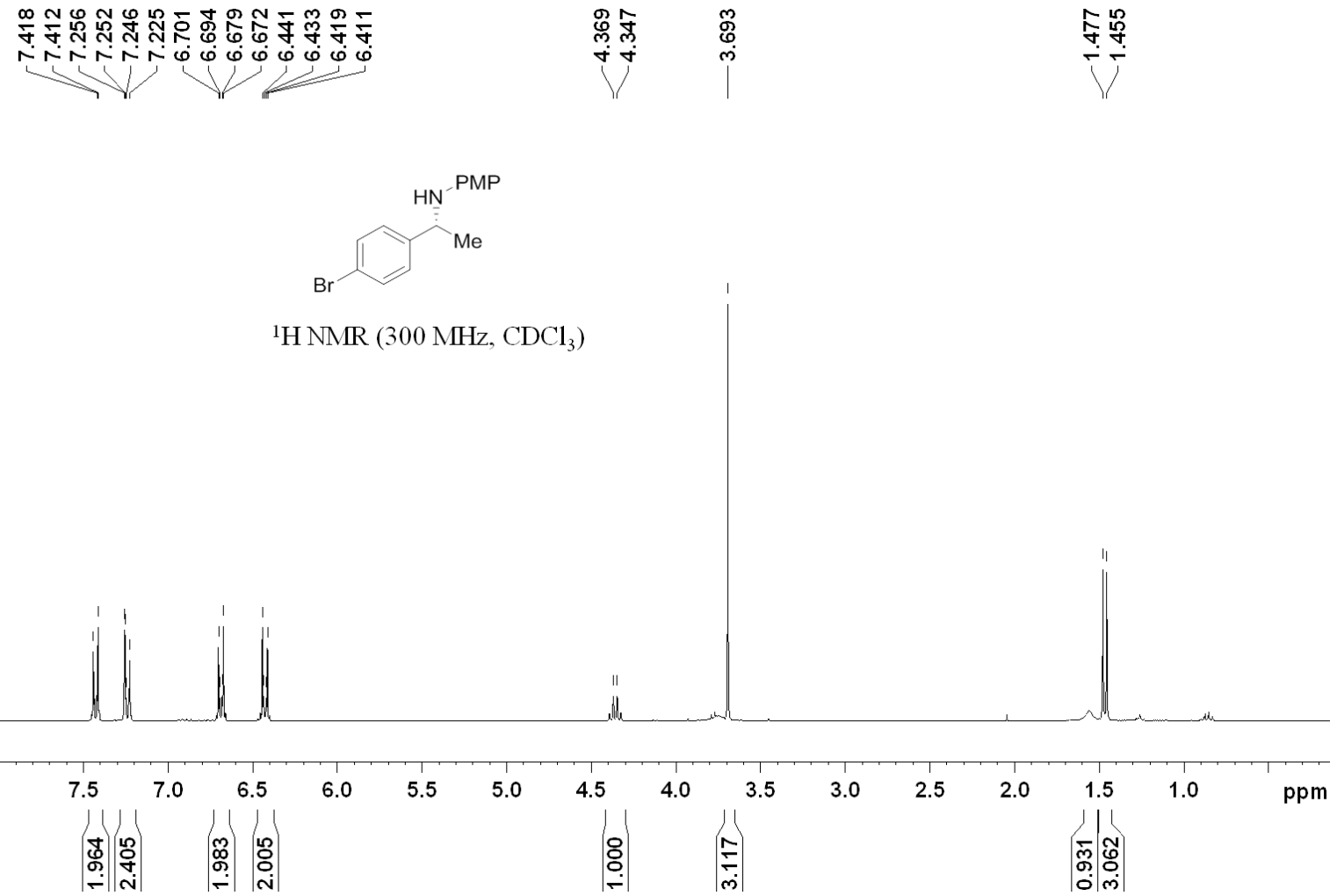

|

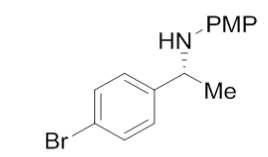

${ }^{13} \mathrm{CNMR}\left(75 \mathrm{MHz}, \mathrm{CDCl}_{3}\right)$

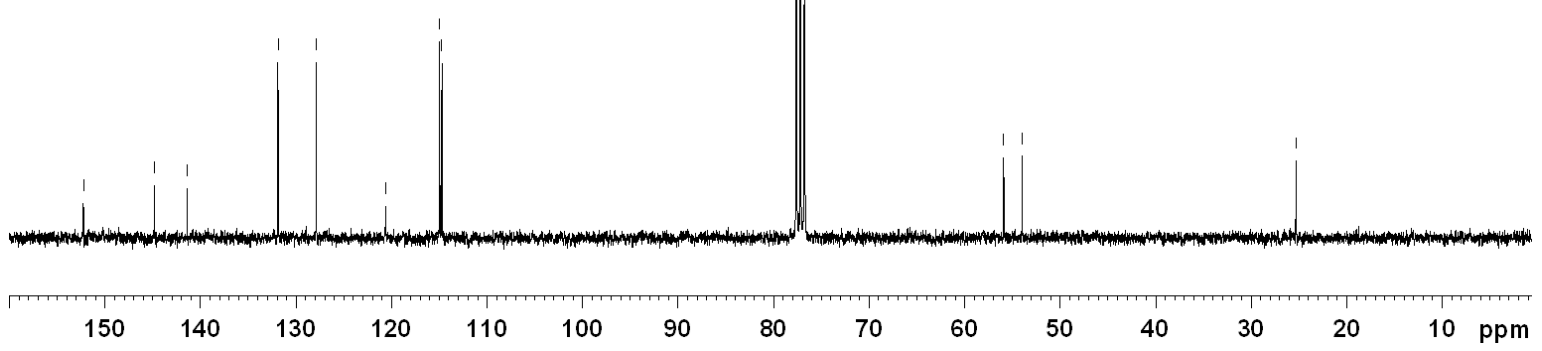


(R)-N-[(1-(p-Nitrophenyl)ethyl]-p-methoxyaniline, $37 R$.

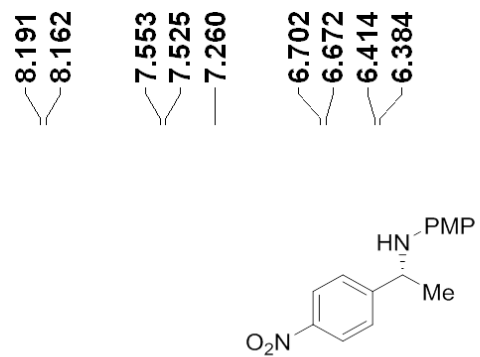

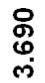

${ }^{1} \mathrm{H} \mathrm{NMR}\left(300 \mathrm{MHz}, \mathrm{CDCl}_{3}\right)$

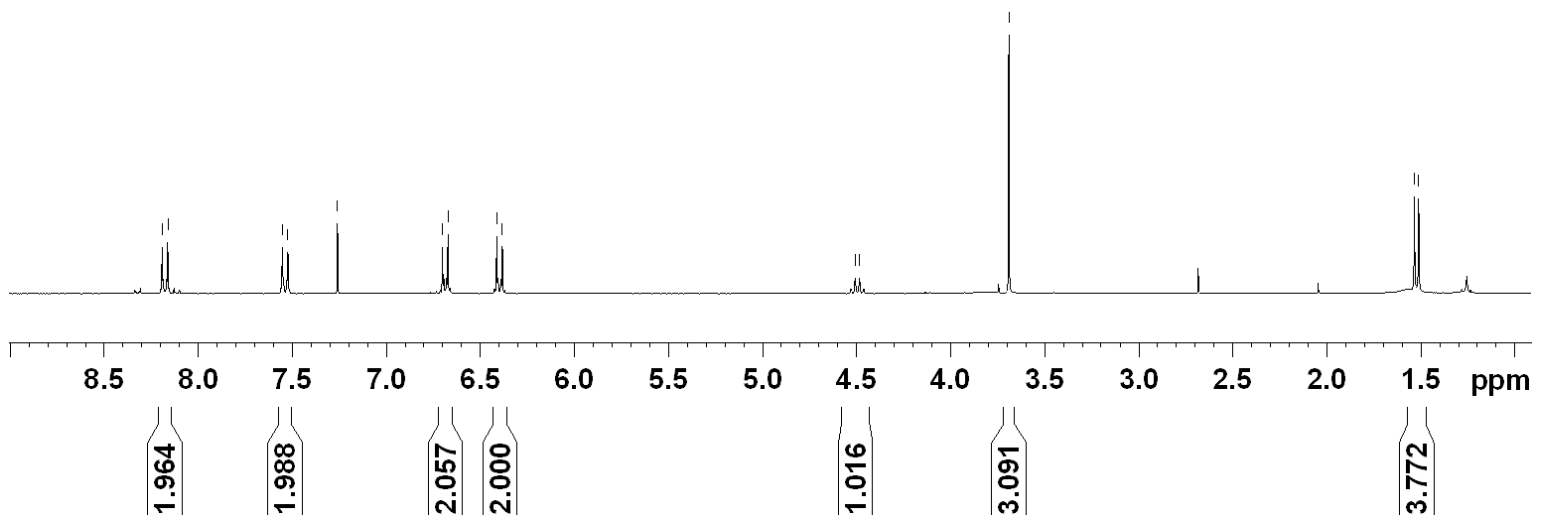

(R)- $N$-[(1-(2'-Naphthyl)ethyl]aniline, 38R

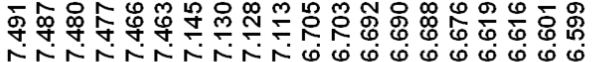

Nㅛ용

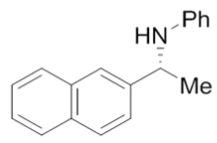

${ }^{1} \mathrm{H} \mathrm{NMR}\left(500 \mathrm{MHz}, \mathrm{CDCl}_{3}\right)$

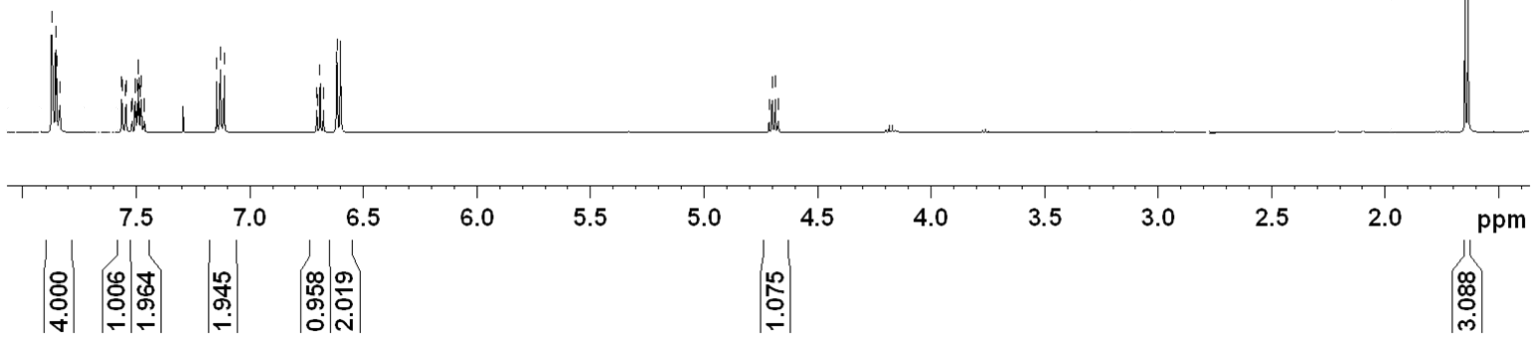




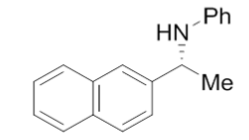

${ }^{13} \mathrm{CNMR}\left(125 \mathrm{MHz}, \mathrm{CDCl}_{3}\right)$

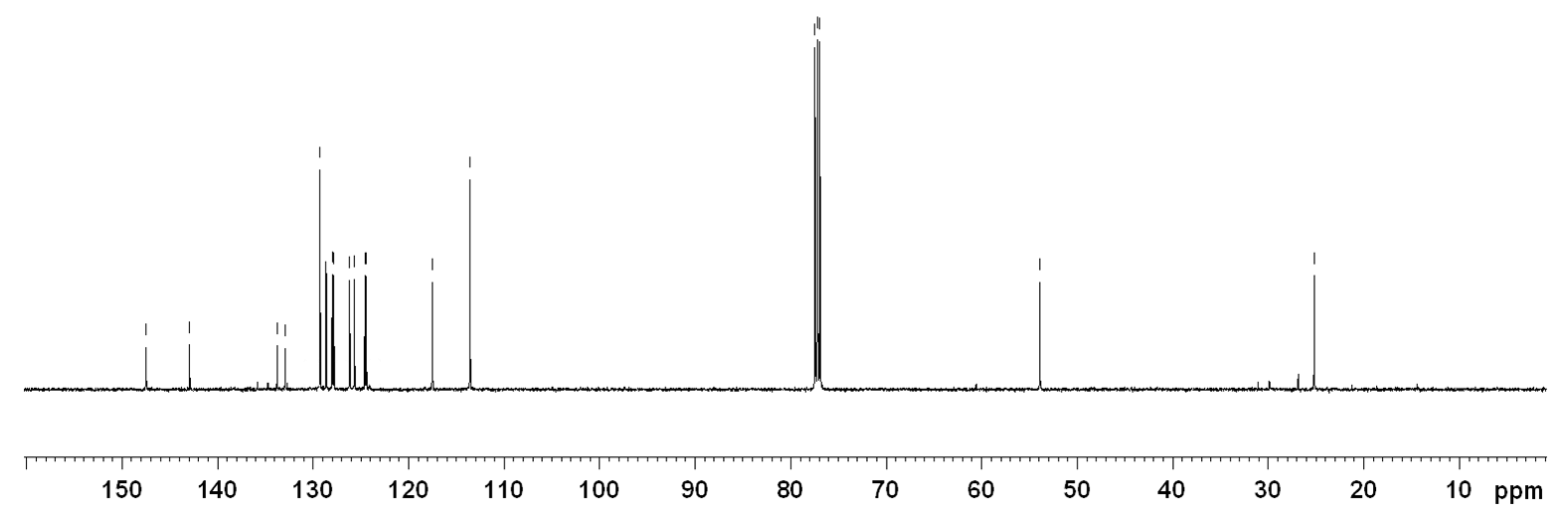

(R)-N-[1-(2'-Naphthyl)ethyl]-p-methoxyaniline, 39R.
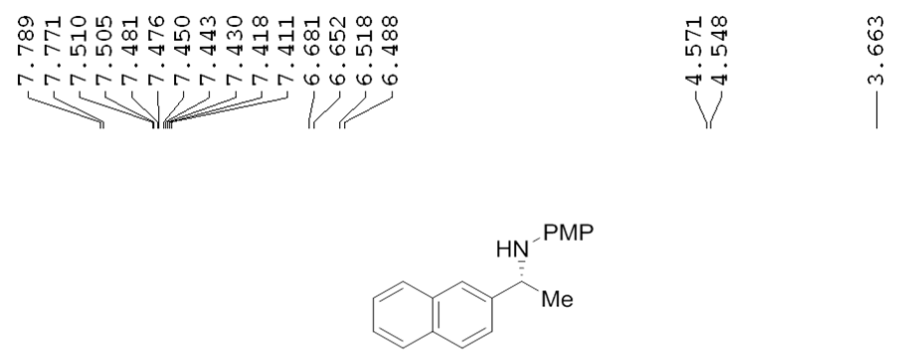

${ }^{1} \mathrm{H} \mathrm{NMR}\left(300 \mathrm{MHz}, \mathrm{CDCl}_{3}\right)$

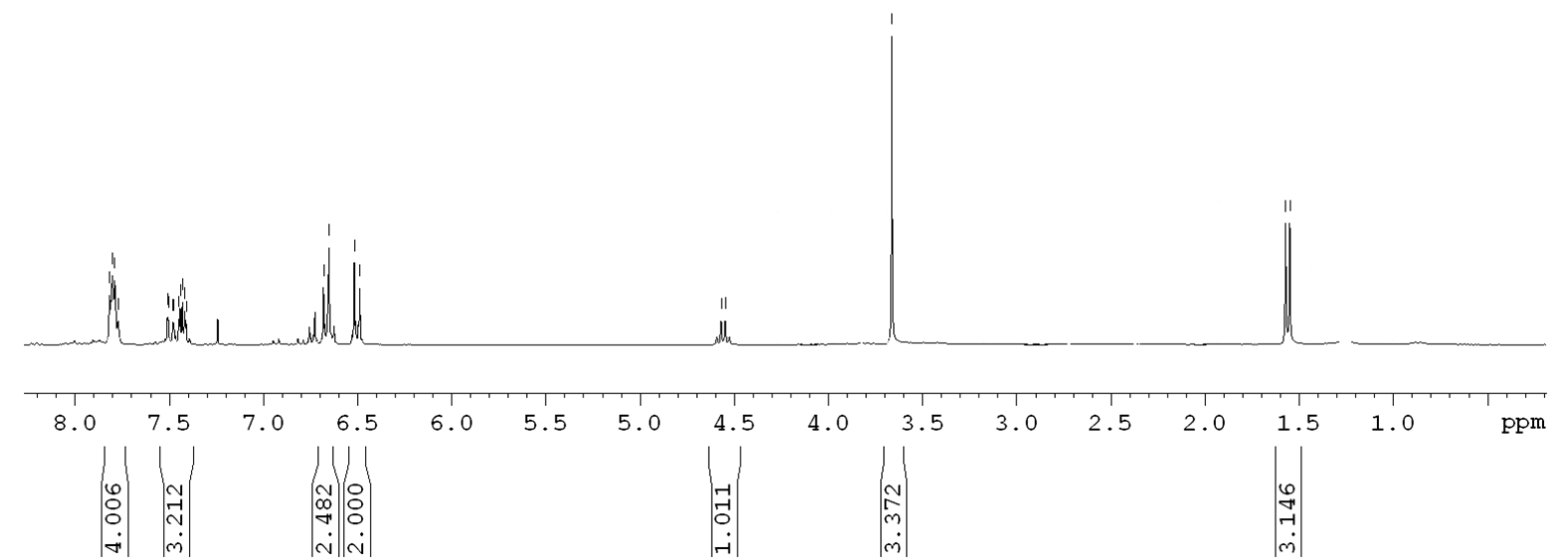


(R)-1-(3-Methylphenyl) ethylamine, 40.

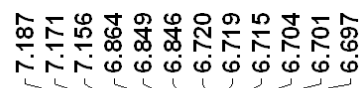

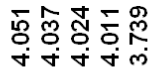

$\rightarrow \infty$

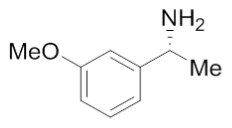

${ }^{1} \mathrm{H} \mathrm{NMR}\left(500 \mathrm{MHz}, \mathrm{CDCl}_{3}\right)$

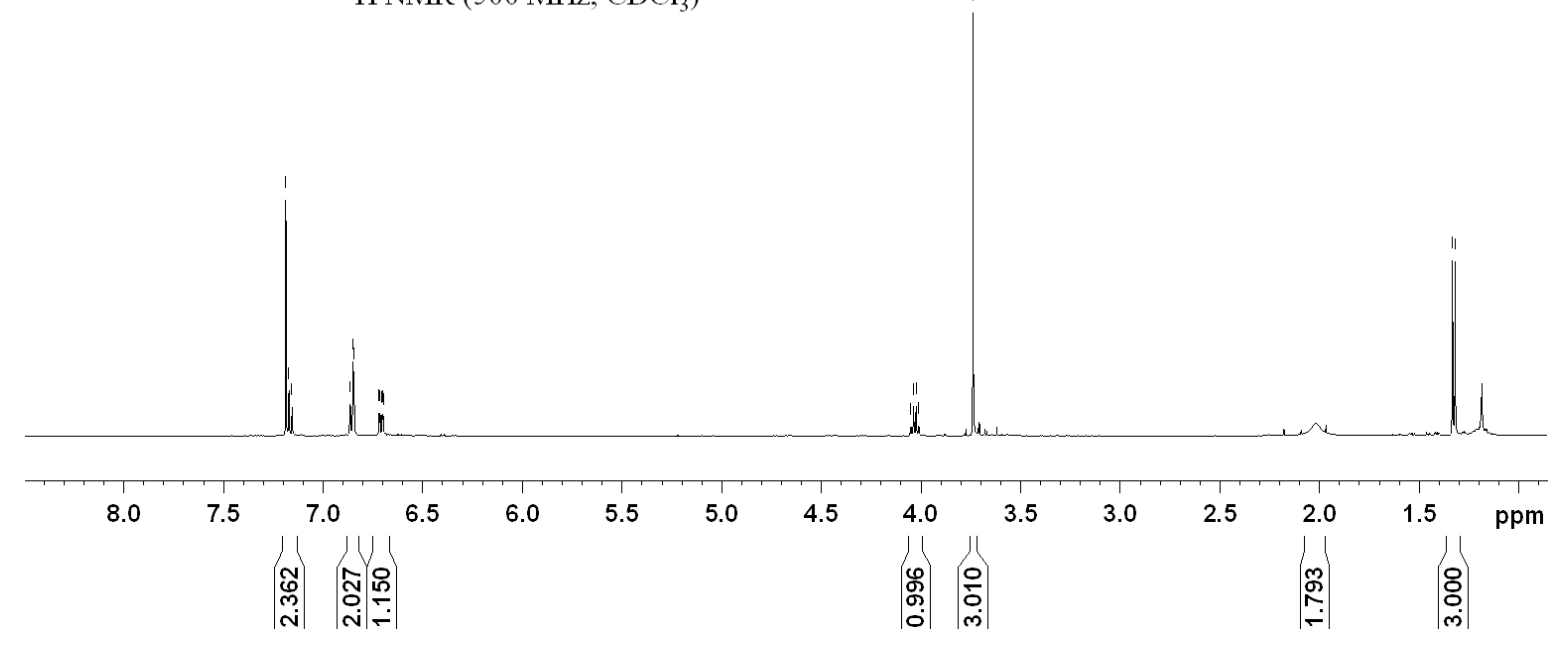



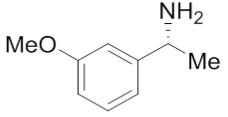

${ }^{13} \mathrm{CNMR}\left(125 \mathrm{MHz}, \mathrm{CDCl}_{3}\right)$

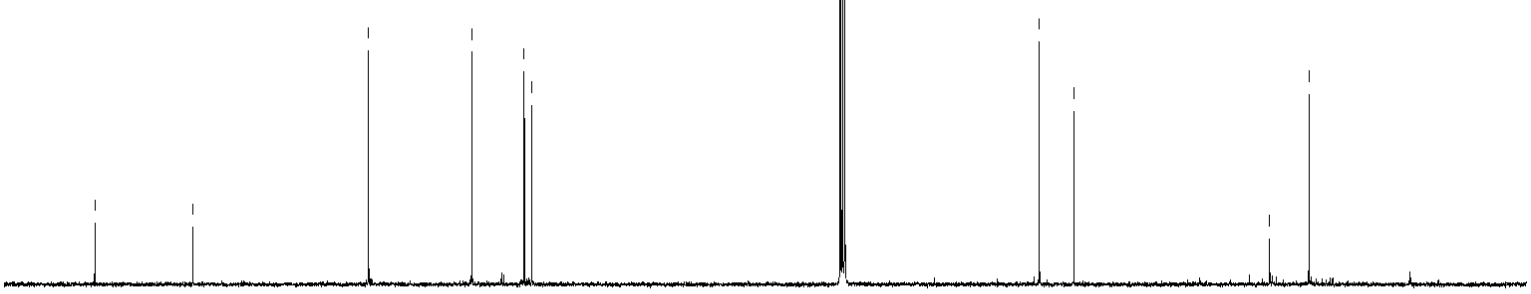

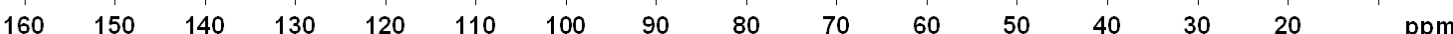


(R)-N-[1-(3-Methoxyphenyl)ethyl]-3-(2-chlorophenyl)-1-propylamine, NPS R-568.

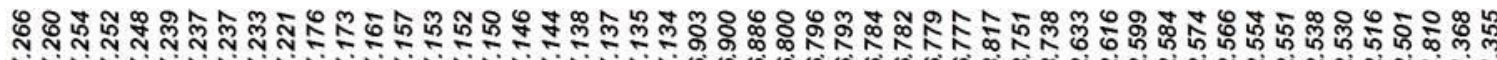

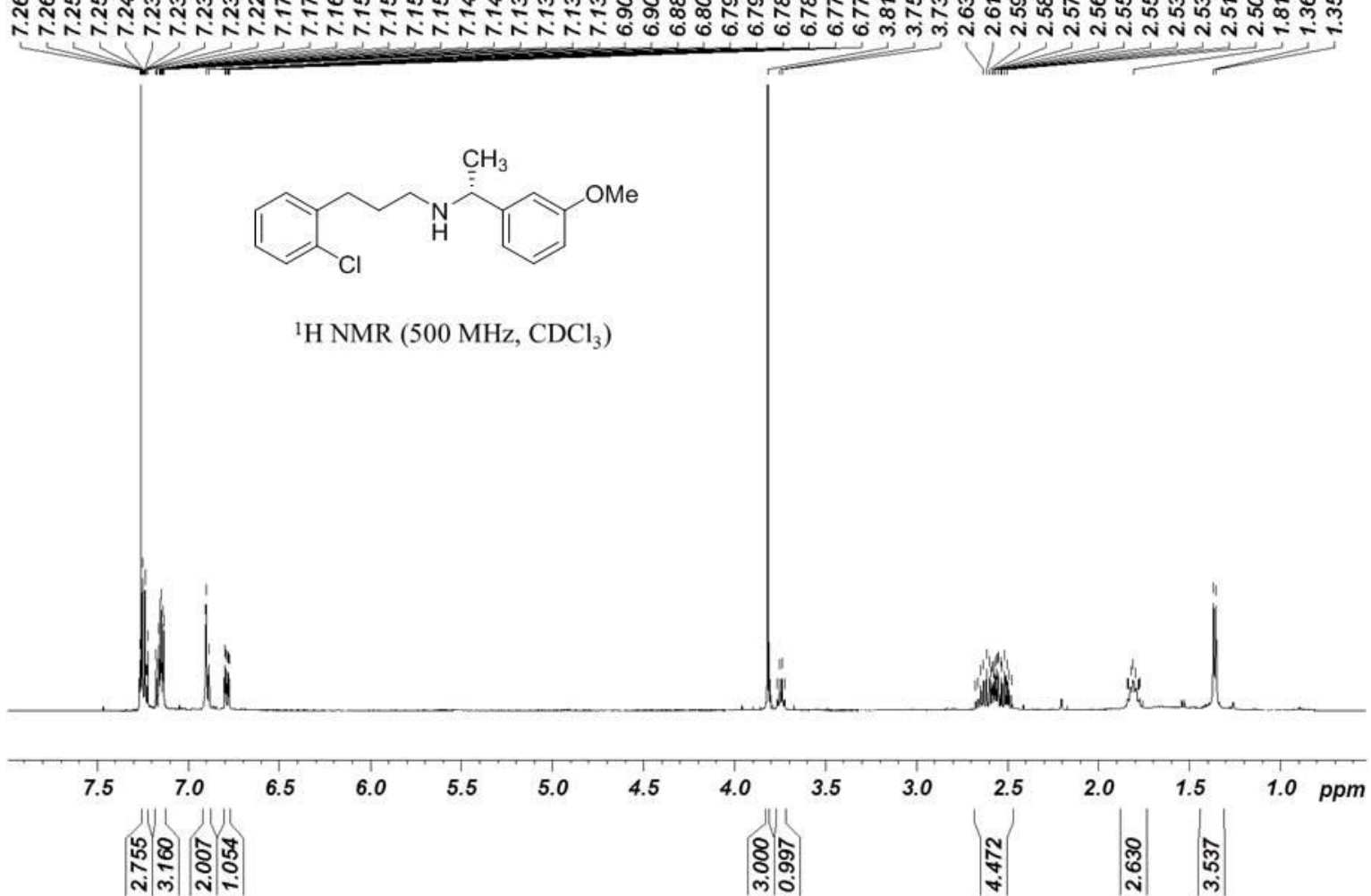

产

సุํำ

Кर官

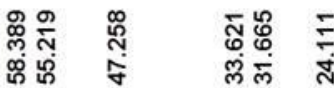

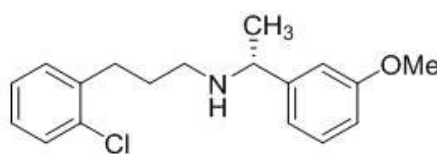

${ }^{13} \mathrm{C} \mathrm{NMR}\left(125 \mathrm{MHz}, \mathrm{CDCl}_{3}\right)$

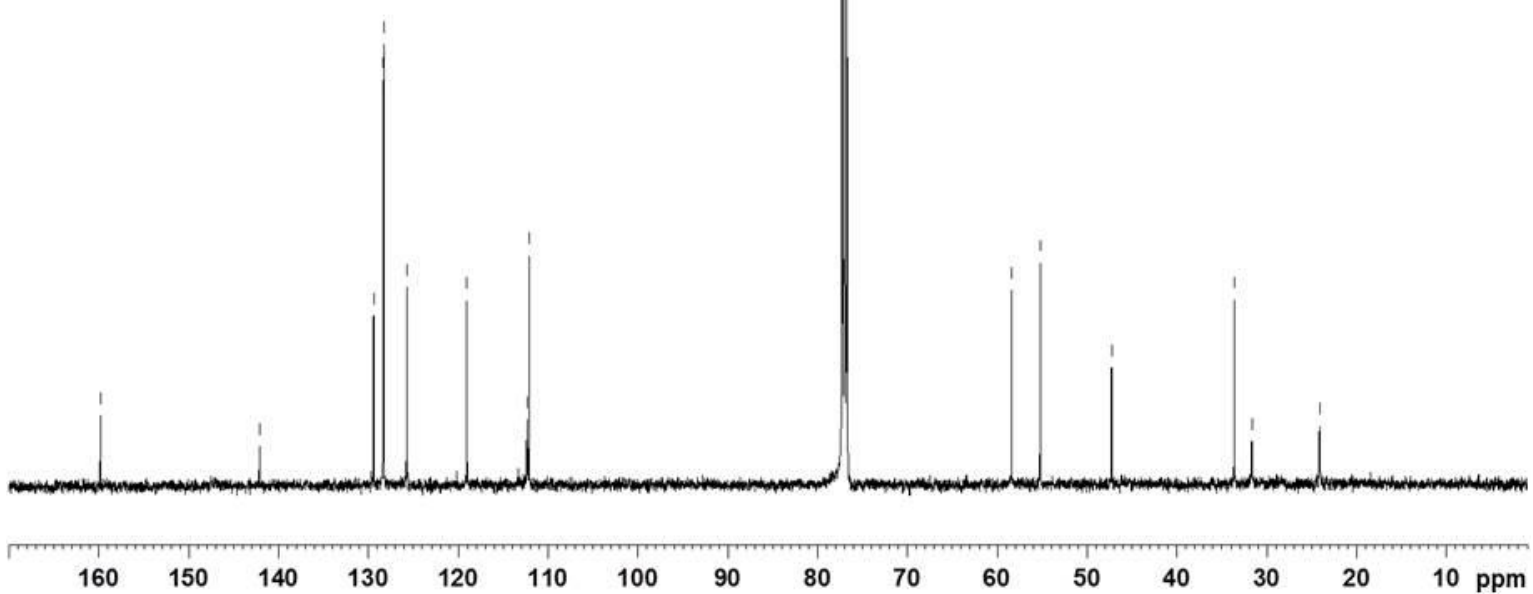


HPLC chromatograms of selected compounds.
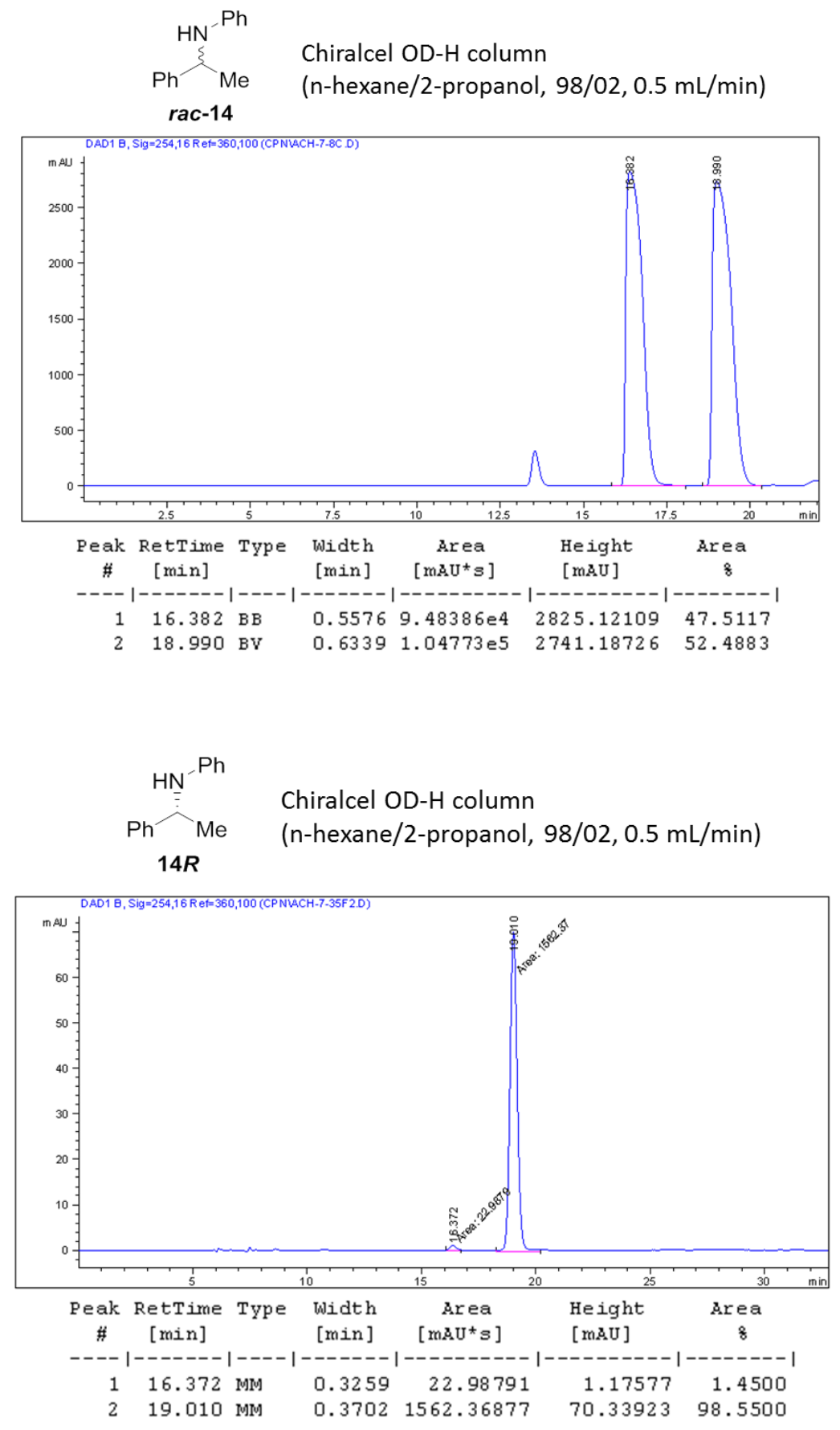

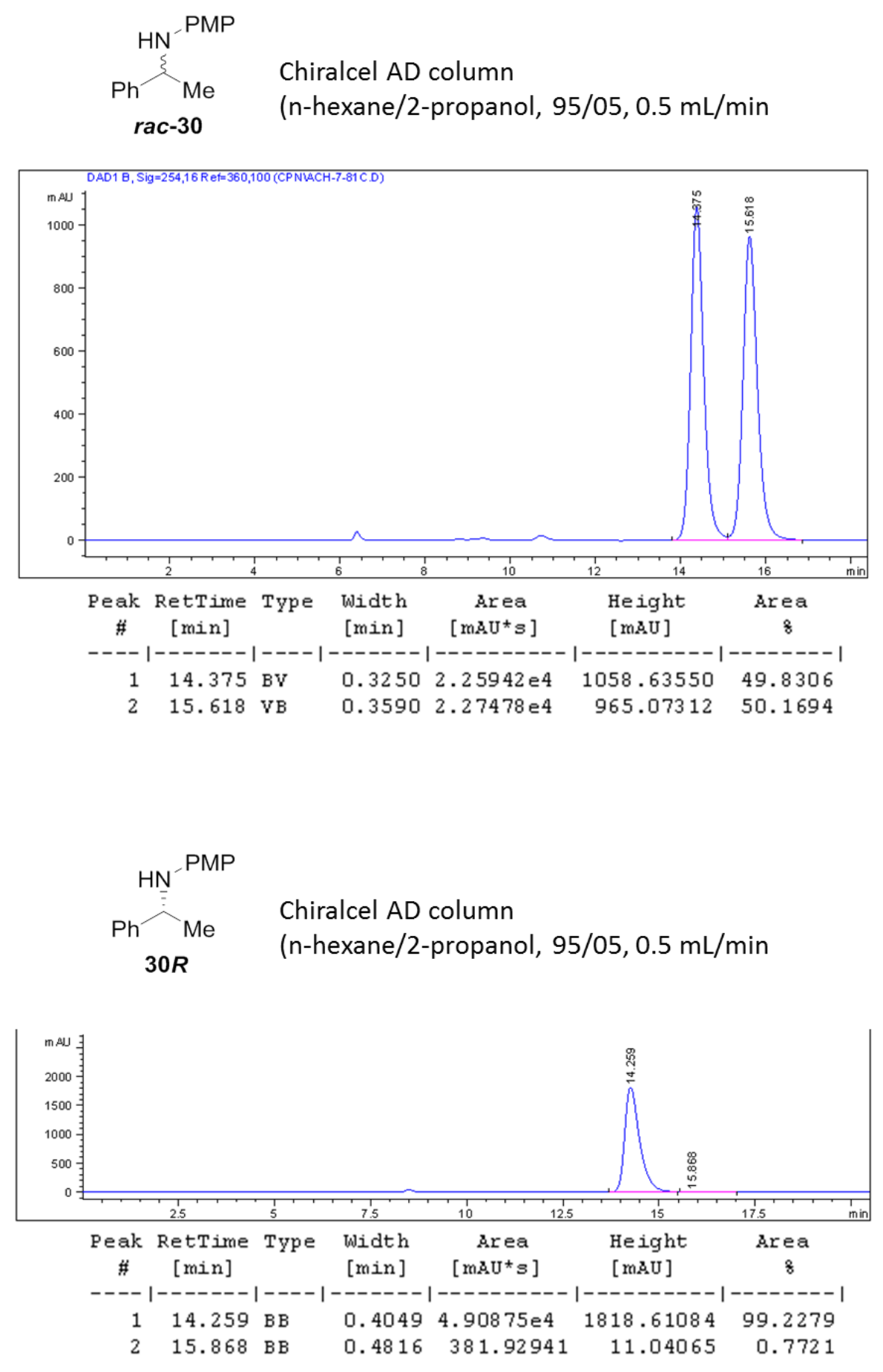
<smiles>COc1ccc(C(C)Nc2ccccc2)cc1</smiles>

Chiralcel OD-H column

(n-hexane/2-propanol, 98/02, $0.5 \mathrm{~mL} / \mathrm{min}$

DAD1 B, Sig $=254,16$ Ref $=360,100$ (CPNIACH-7-30F 2. D)
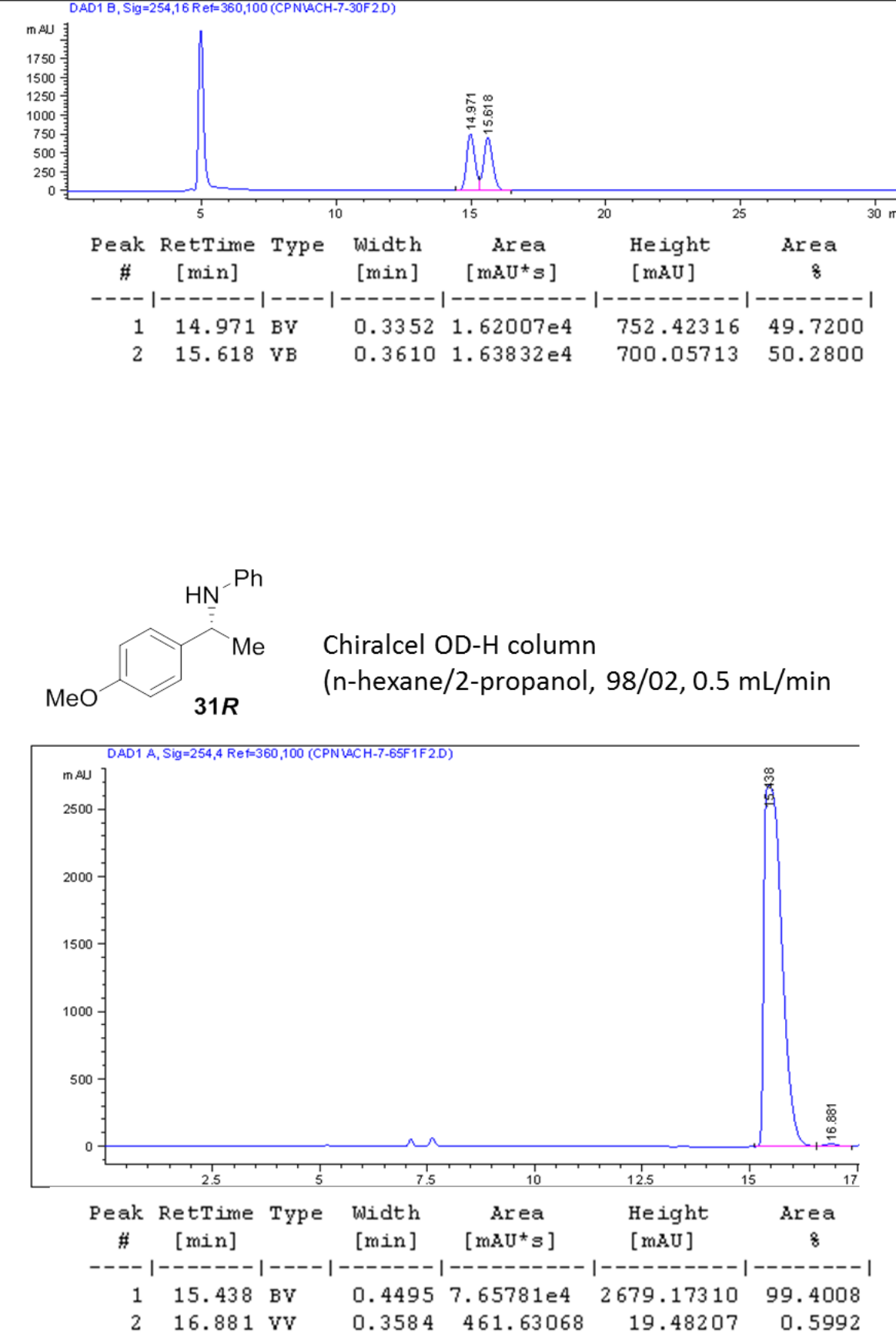

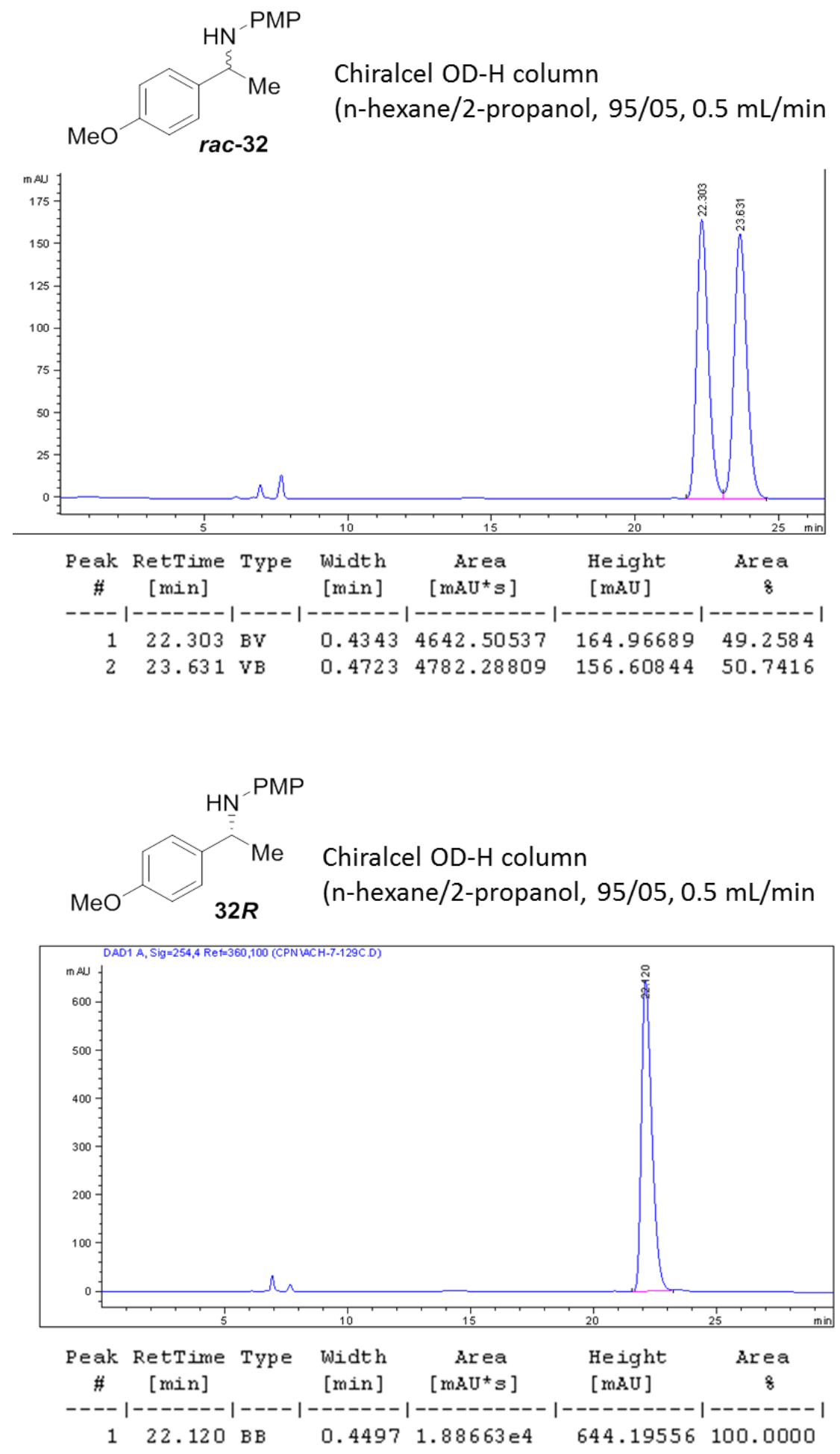


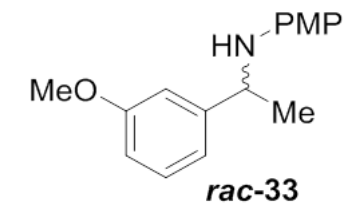

Chiralcel OD-H column

(n-hexane/2-propanol, 96/04, $0.5 \mathrm{~mL} / \mathrm{min}$

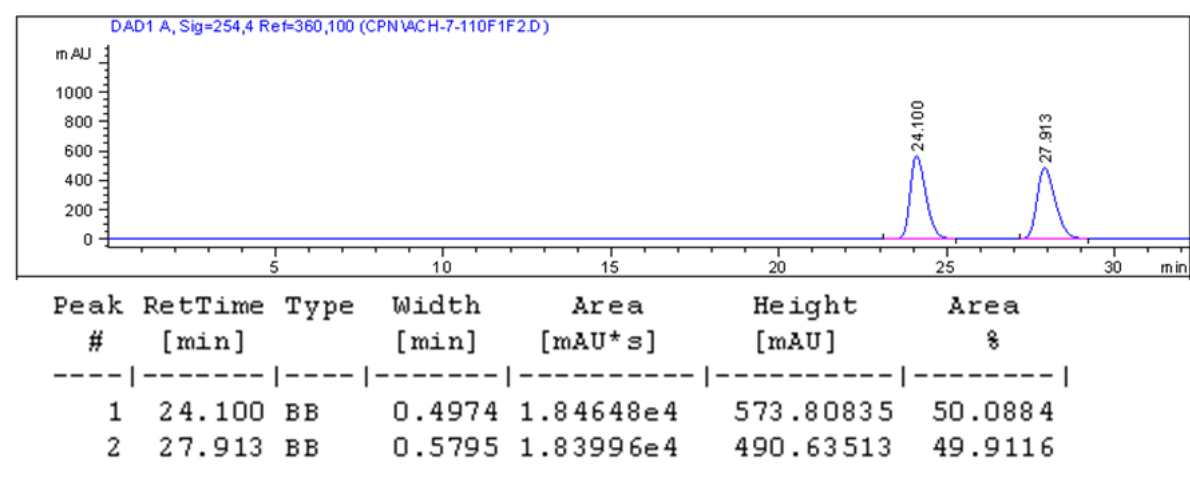

$\mathrm{HN}^{-\mathrm{PMP}}$

$\mathrm{MeO}$<smiles>CCc1ccccc1</smiles>

Chiralcel OD-H column

(n-hexane/2-propanol, 96/04, $0.5 \mathrm{~mL} / \mathrm{min}$

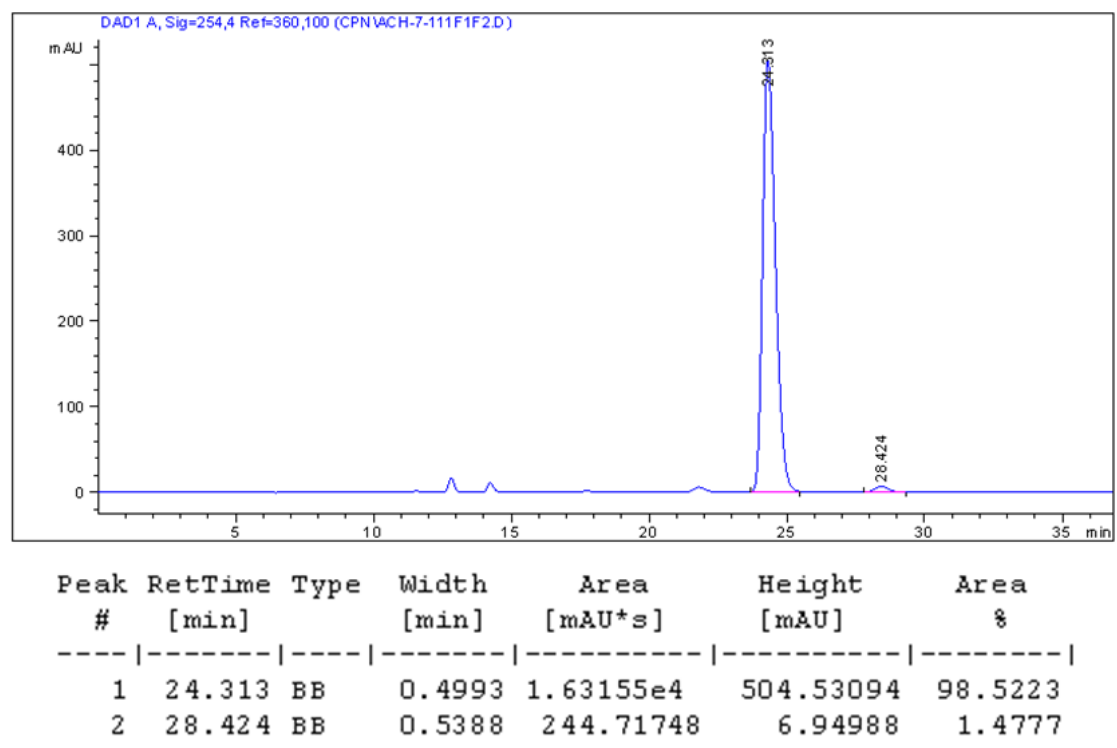



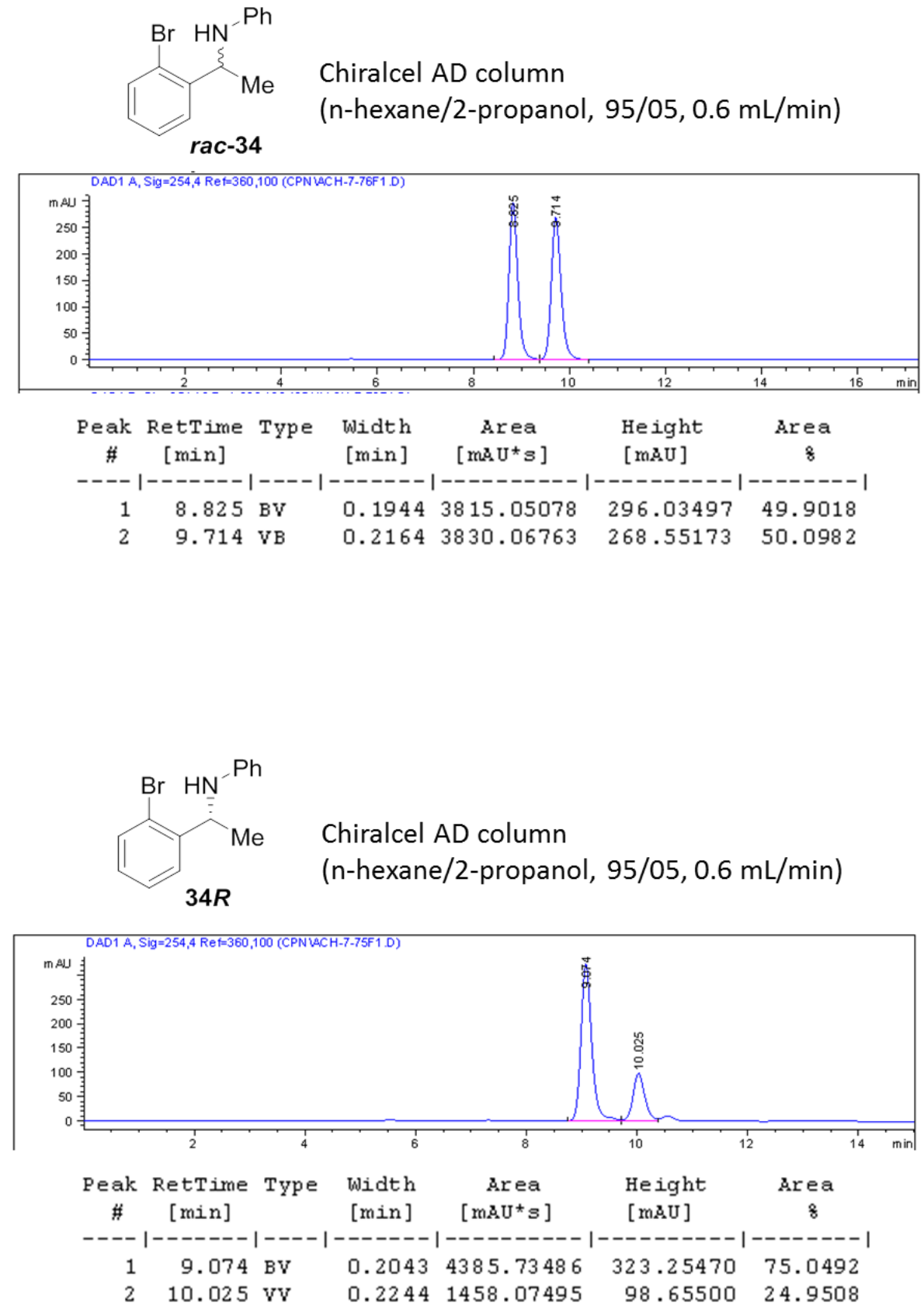


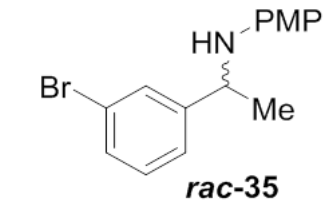

Chiralcel AD column

(n-hexane/2-propanol, 98/02, $0.6 \mathrm{~mL} / \mathrm{min}$ )
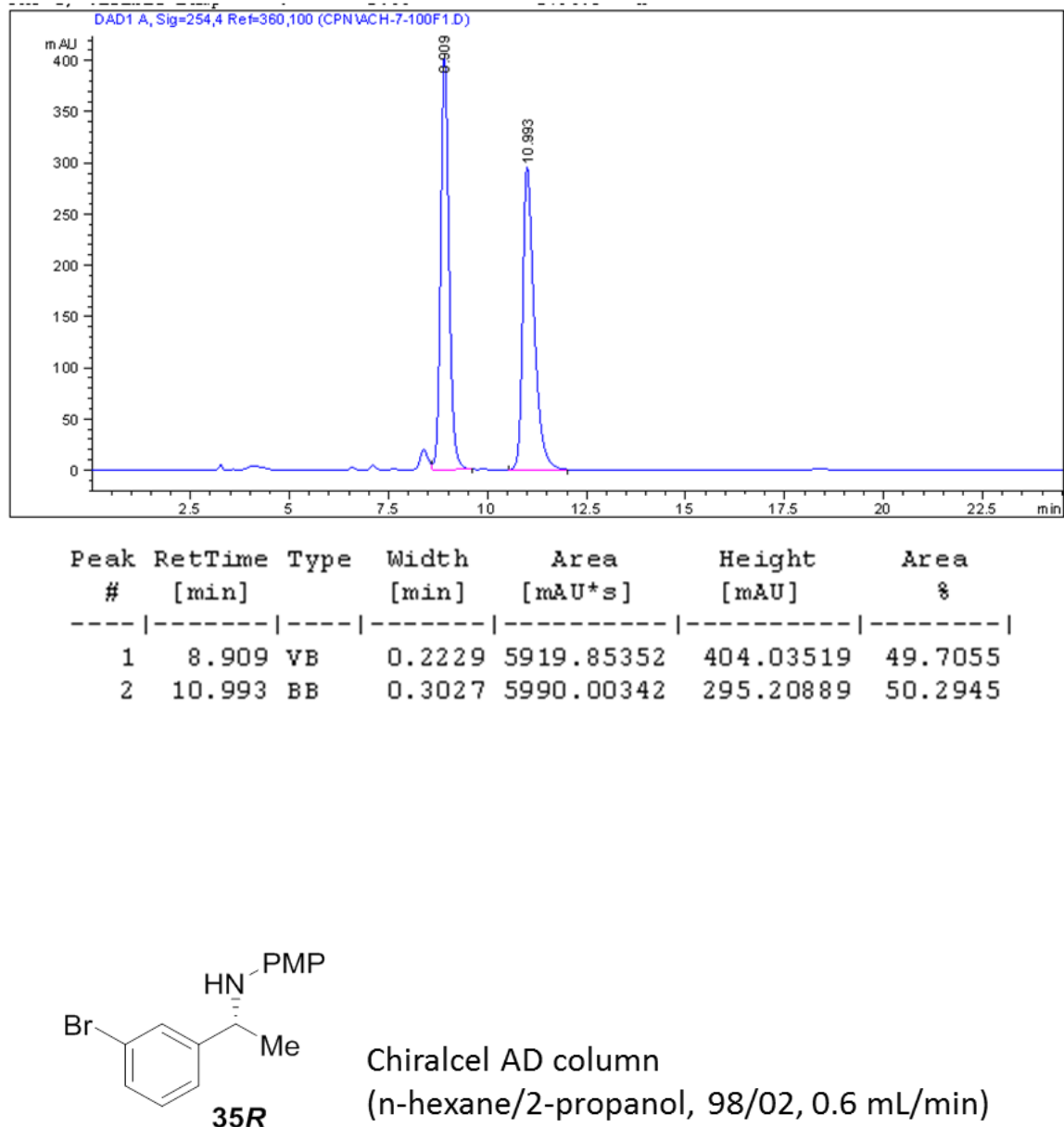

Chiralcel AD column

(n-hexane/2-propanol, 98/02, $0.6 \mathrm{~mL} / \mathrm{min}$ )

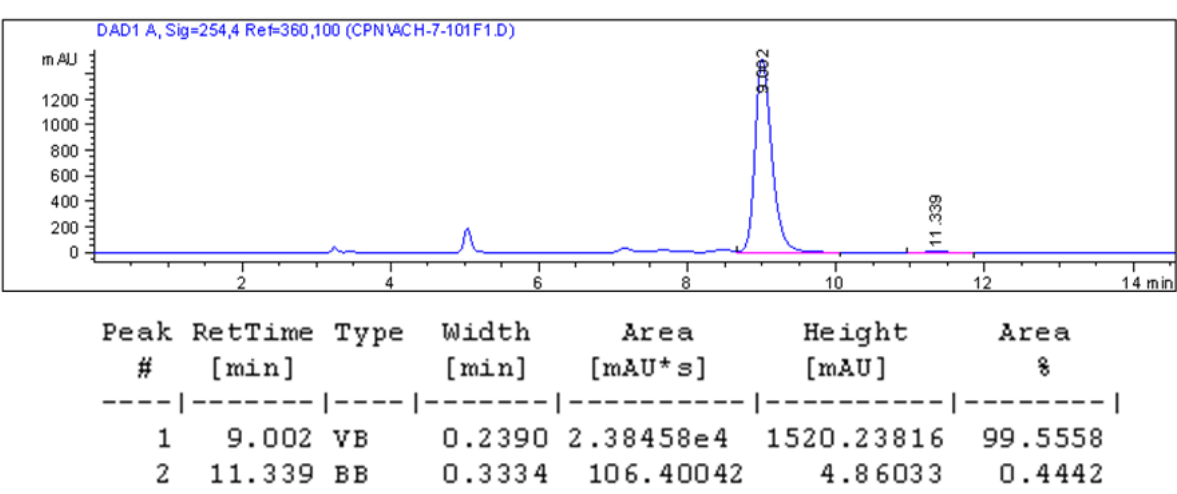


<smiles>CC(NP)c1ccc(Br)cc1</smiles>

Chiralcel OD-H column

(n-hexane/2-propanol, 98/02, $0.6 \mathrm{~mL} / \mathrm{min}$
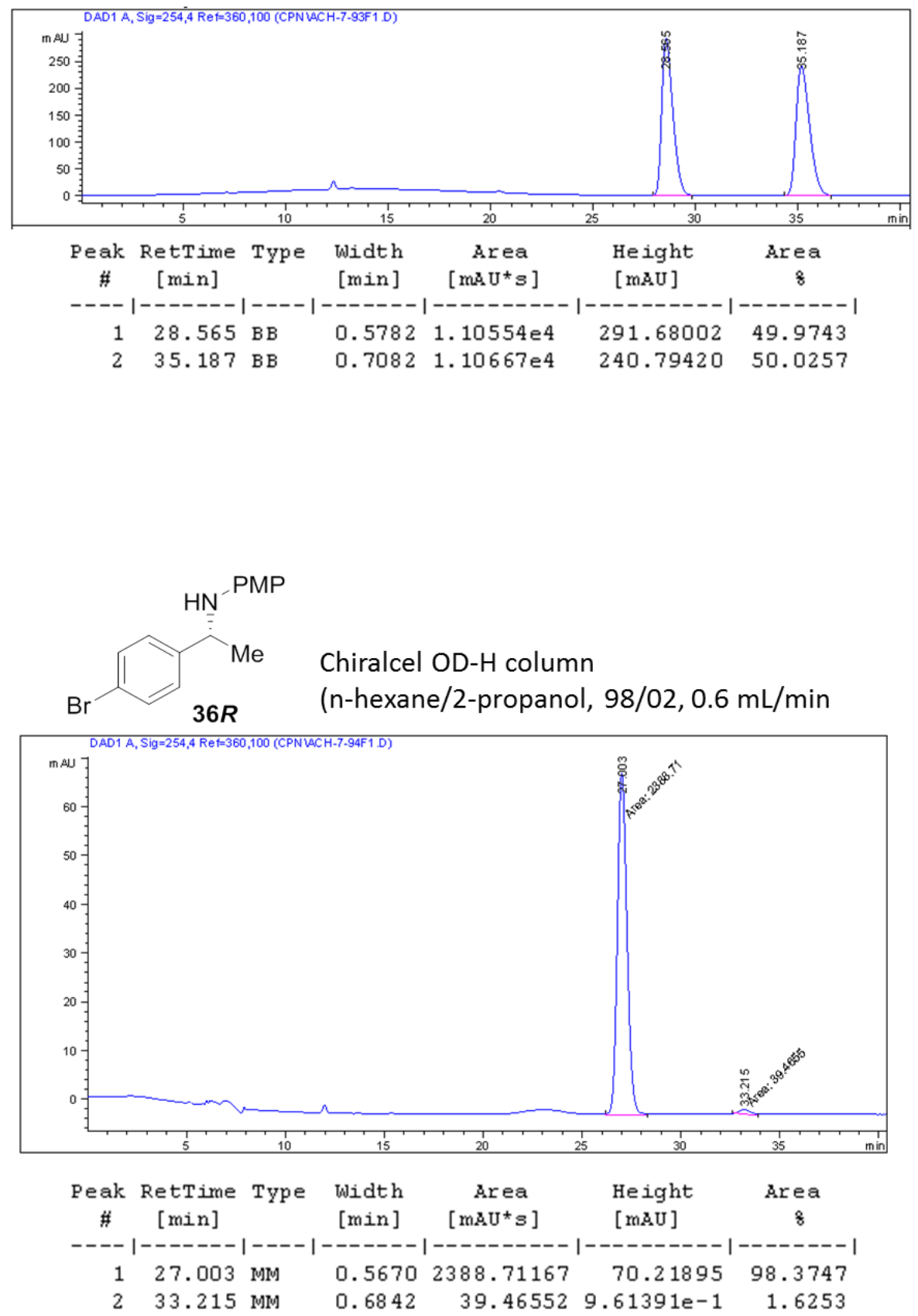

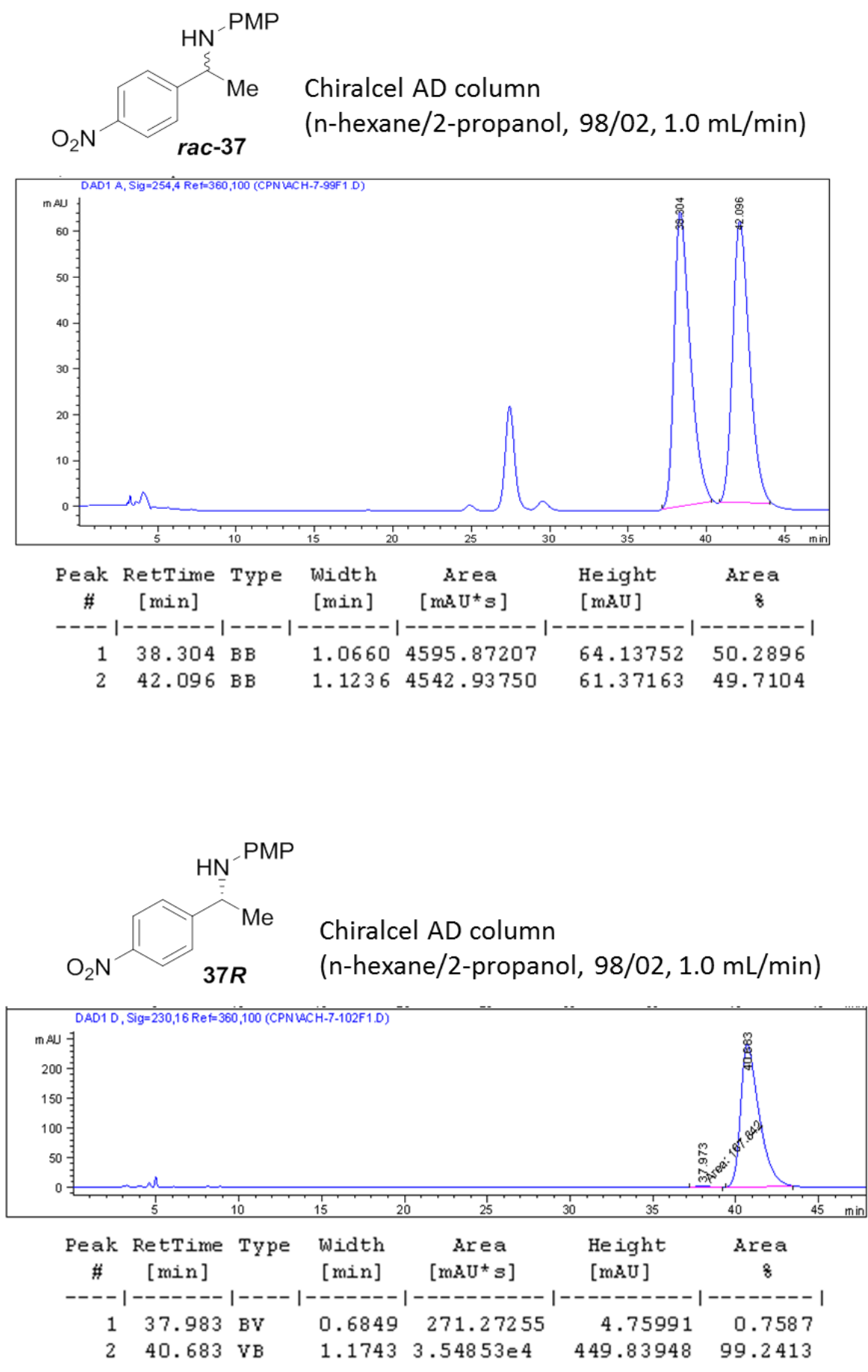

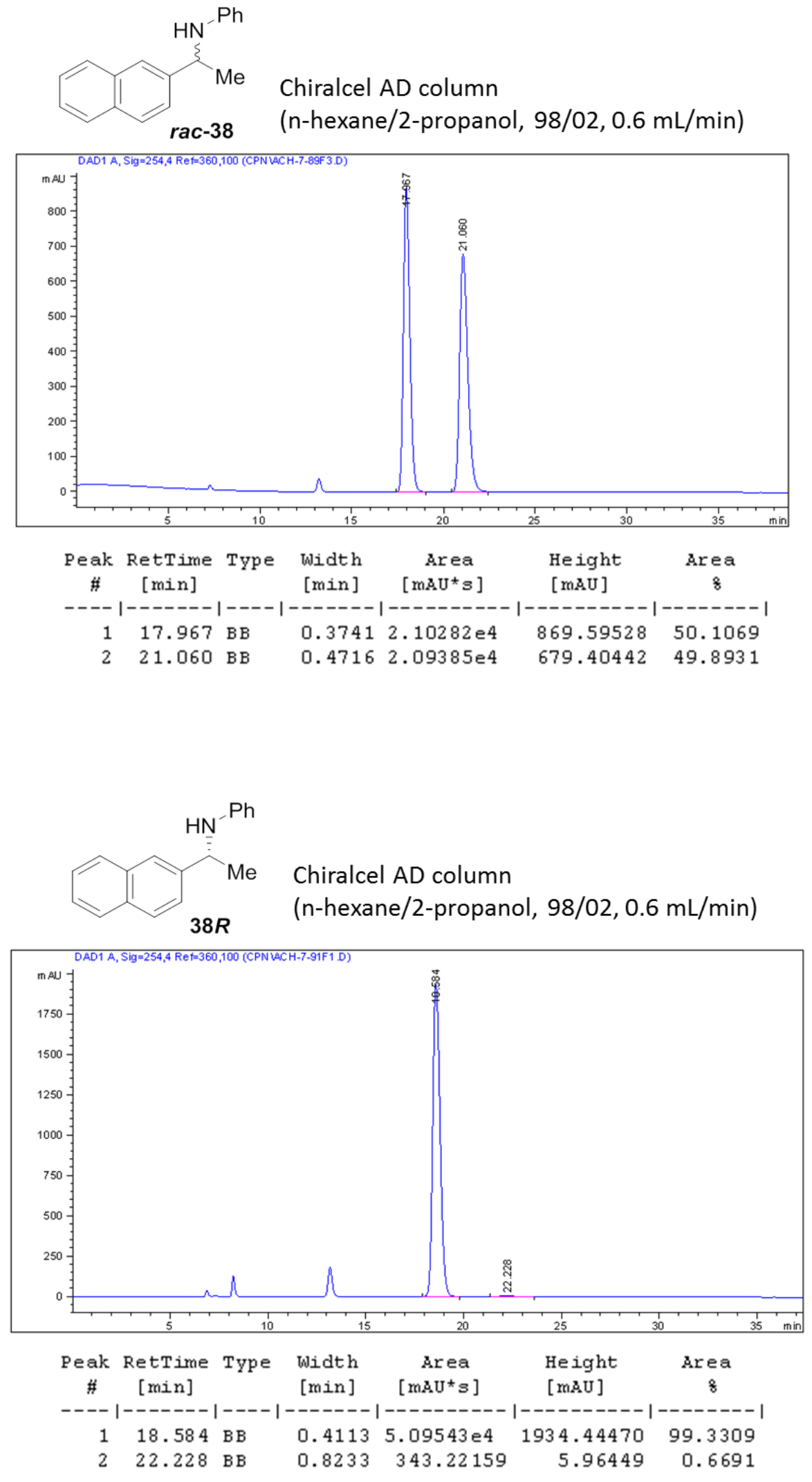

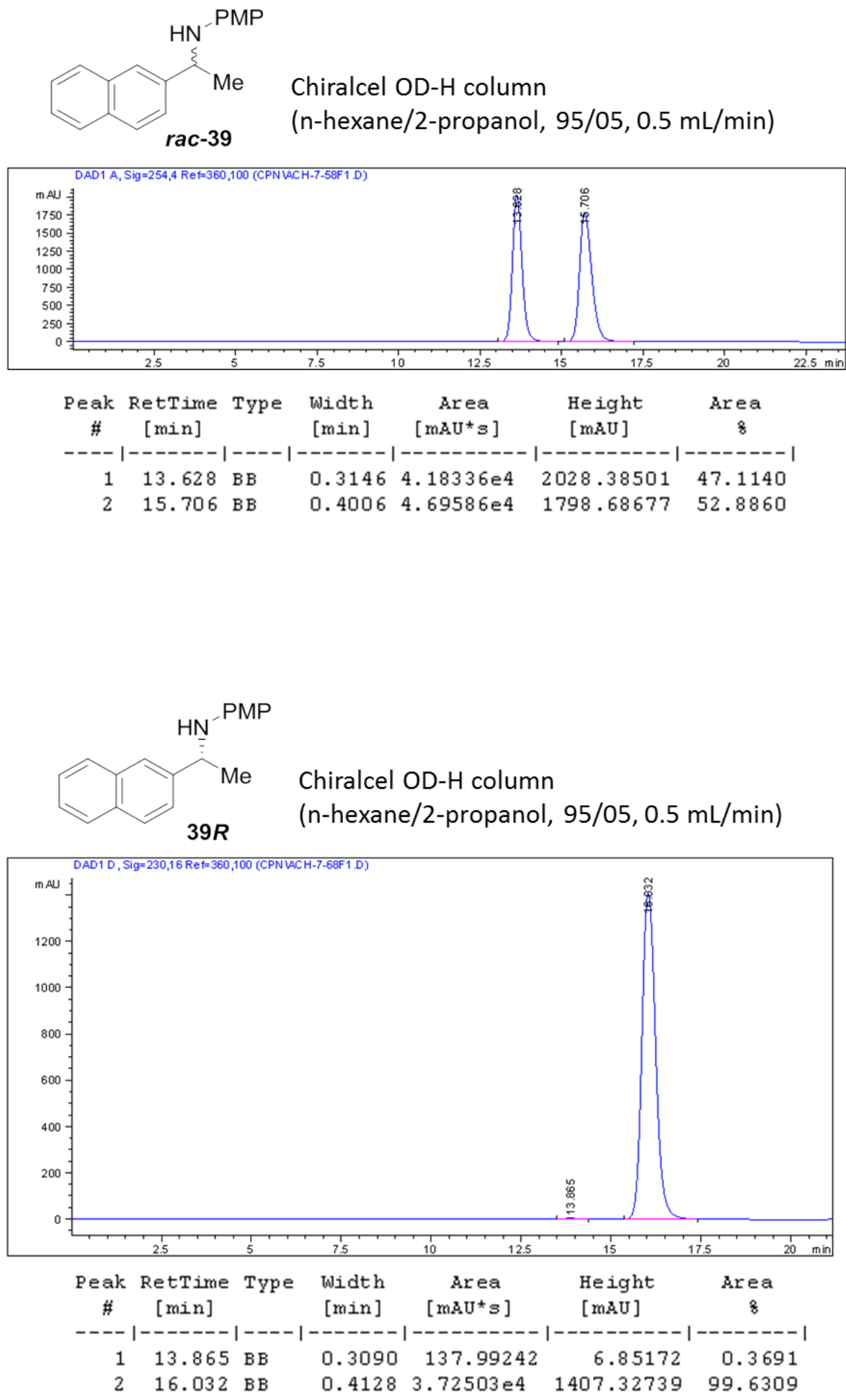
Crystallography Data for selected compounds.

Methyl 2-(tert-butylsulfinamide)-2-[3,5-bis(trifluoromethyl)phenyl]ethyl phenylphosphinate, $9(R, S, S)$.

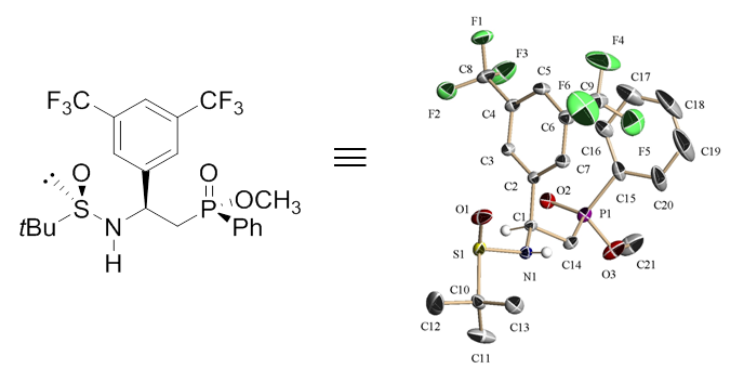

Table 1. Crystal data and structure refinement for compound $9(R, S, S)$.

Empirical formula

Formula weight

Temperature

Wavelength

Crystal system

Space group

Unit cell dimensions

Volume

Z

Density (calculated)

Absorption coefficient

$\mathrm{F}(000)$

Crystal size

Theta range for data collection

Index ranges

Reflections collected

Independent reflections

Completeness to theta $=25.25^{\circ}$

Absorption correction

Max. and min. transmission

Refinement method

Data / restraints / parameters
$\mathrm{C}_{21} \mathrm{H}_{24} \mathrm{~F}_{6} \mathrm{NO}_{3} \mathrm{PS}$

515.44

213(2) K

$0.71073 \AA$

Orthorhombic

P $22_{1} 2_{1} 2_{1}$

$\mathrm{a}=10.9093(5) \AA$ $\alpha=90^{\circ}$.

$\mathrm{b}=13.3000(7) \AA$ $\beta=90^{\circ}$.

$\mathrm{c}=17.2973(8) \AA$ $\gamma=90^{\circ}$
4

$1.364 \mathrm{Mg} / \mathrm{m}^{3}$

$0.259 \mathrm{~mm}^{-1}$

1064

$0.50 \times 0.45 \times 0.40 \mathrm{~mm}^{3}$

3.37 to $25.25^{\circ}$.

$-13<=\mathrm{h}<=12,-15<=\mathrm{k}<=10,-20<=\mathrm{l}<=17$

22834

$4475[\mathrm{R}(\mathrm{int})=0.0270]$

$99.6 \%$

Semi-empirical from equivalents

0.9035 and 0.8814

Full-matrix least-squares on $\mathrm{F}^{2}$

4475 / 37 / 305 
Goodness-of-fit on $\mathrm{F}^{2}$

Final $\mathrm{R}$ indices [I $>2 \operatorname{sigma}(\mathrm{I})]$

$\mathrm{R}$ indices (all data)

Absolute structure parameter

Largest diff. peak and hole
1.068

$\mathrm{R} 1=0.0546, \mathrm{wR} 2=0.1595$

$\mathrm{R} 1=0.0579, \mathrm{wR} 2=0.1636$

0.04(8)

0.535 and -0.404 e. $\AA^{-3}$

Table 2. Atomic coordinates $\left(\times 10^{4}\right)$ and equivalent isotropic displacement parameters $\left(\AA^{2} \times 10^{3}\right)$ for compound $\mathbf{9}(\boldsymbol{R}, S, S)$. U(eq) is defined as one third of the trace of the orthogonalized $\mathrm{U}^{\mathrm{ij}}$ tensor.

\begin{tabular}{|c|c|c|c|c|}
\hline & $\mathrm{x}$ & $\mathrm{y}$ & $\mathrm{z}$ & $\mathrm{U}(\mathrm{eq})$ \\
\hline$S(1)$ & $5067(1)$ & $10424(1)$ & $5415(1)$ & $38(1)$ \\
\hline $\mathrm{P}(1)$ & $5808(1)$ & $6482(1)$ & $4663(1)$ & $33(1)$ \\
\hline $\mathrm{O}(1)$ & $4462(5)$ & 10586(3) & $6171(2)$ & $62(1)$ \\
\hline $\mathrm{O}(2)$ & $7126(2)$ & $6585(2)$ & $4827(2)$ & $39(1)$ \\
\hline $\mathrm{O}(3)$ & $5462(4)$ & $6046(3)$ & $3844(2)$ & $62(1)$ \\
\hline $\mathrm{N}(1)$ & $4619(3)$ & $9345(2)$ & $5019(2)$ & $28(1)$ \\
\hline $\mathrm{C}(1)$ & $5398(3)$ & $8473(3)$ & $5171(2)$ & $26(1)$ \\
\hline $\mathrm{C}(2)$ & $5386(4)$ & $8098(3)$ & $6006(2)$ & $28(1)$ \\
\hline$C(3)$ & $6488(4)$ & $7928(3)$ & $6386(2)$ & $30(1)$ \\
\hline $\mathrm{C}(4)$ & $6497(4)$ & $7523(3)$ & $7126(2)$ & $35(1)$ \\
\hline$C(5)$ & $5427(4)$ & 7294(4) & 7501(3) & $42(1)$ \\
\hline$C(6)$ & 4319(4) & 7501(3) & $7127(3)$ & $39(1)$ \\
\hline$C(7)$ & $4300(4)$ & $7895(3)$ & 6391(2) & $35(1)$ \\
\hline $\mathrm{C}(8)$ & $7713(5)$ & $7292(4)$ & $7487(3)$ & $50(1)$ \\
\hline $\mathrm{F}(1)$ & $7670(3)$ & 7197(3) & $8239(2)$ & $73(1)$ \\
\hline $\mathrm{F}(2)$ & $8549(3)$ & $7980(3)$ & $7338(2)$ & $89(1)$ \\
\hline $\mathrm{F}(3)$ & $8187(4)$ & $6440(4)$ & $7212(3)$ & $103(2)$ \\
\hline $\mathrm{C}(9)$ & $3135(5)$ & $7306(5)$ & $7526(3)$ & $64(2)$ \\
\hline $\mathrm{F}(4)$ & $3209(5)$ & $6837(7)$ & $8155(4)$ & $159(2)$ \\
\hline $\mathrm{F}(5)$ & 2281(4) & $6966(6)$ & $7078(3)$ & $122(2)$ \\
\hline $\mathrm{F}(6)$ & $2607(6)$ & $8169(6)$ & $7734(4)$ & $153(2)$ \\
\hline$C(10)$ & $4321(4)$ & $11300(3)$ & $4735(3)$ & $40(1)$ \\
\hline $\mathrm{C}(11)$ & $4777(7)$ & $11087(6)$ & 3931(4) & $80(2)$ \\
\hline$C(12)$ & $4746(9)$ & $12330(4)$ & $5010(6)$ & $105(3)$ \\
\hline$C(13)$ & $2959(4)$ & $11196(4)$ & $4793(4)$ & $55(1)$ \\
\hline
\end{tabular}




\begin{tabular}{lrlrr}
$\mathrm{C}(14)$ & $4986(3)$ & $7657(3)$ & $4601(2)$ & $31(1)$ \\
$\mathrm{C}(15)$ & $5067(4)$ & $5691(3)$ & $5350(3)$ & $44(1)$ \\
$\mathrm{C}(16)$ & $5644(5)$ & $5474(4)$ & $6052(3)$ & $50(1)$ \\
$\mathrm{C}(17)$ & $5008(8)$ & $4930(5)$ & $6618(5)$ & $92(2)$ \\
$\mathrm{C}(18)$ & $3825(9)$ & $4638(6)$ & $6471(7)$ & $122(4)$ \\
$\mathrm{C}(19)$ & $3266(8)$ & $4840(7)$ & $5788(9)$ & $139(4)$ \\
$\mathrm{C}(20)$ & $3875(5)$ & $5368(5)$ & $5227(6)$ & $84(2)$ \\
$\mathrm{C}(21)$ & $5990(8)$ & $5116(5)$ & $3578(4)$ & $82(2)$ \\
\hline
\end{tabular}

Table 3. Bond lengths $[\AA]$ and angles $\left[{ }^{\circ}\right]$ for compound $\mathbf{9}(\boldsymbol{R}, \boldsymbol{S}, \boldsymbol{S})$.

\begin{tabular}{ll}
\hline $\mathrm{S}(1)-\mathrm{O}(1)$ & $1.480(4)$ \\
$\mathrm{S}(1)-\mathrm{N}(1)$ & $1.663(3)$ \\
$\mathrm{S}(1)-\mathrm{C}(10)$ & $1.845(4)$ \\
$\mathrm{P}(1)-\mathrm{O}(2)$ & $1.473(3)$ \\
$\mathrm{P}(1)-\mathrm{O}(3)$ & $1.576(4)$ \\
$\mathrm{P}(1)-\mathrm{C}(15)$ & $1.781(5)$ \\
$\mathrm{P}(1)-\mathrm{C}(14)$ & $1.805(4)$ \\
$\mathrm{O}(3)-\mathrm{C}(21)$ & $1.440(6)$ \\
$\mathrm{N}(1)-\mathrm{C}(1)$ & $1.463(5)$ \\
$\mathrm{N}(1)-\mathrm{H}(1 \mathrm{~N})$ & $0.83(2)$ \\
$\mathrm{C}(1)-\mathrm{C}(2)$ & $1.528(5)$ \\
$\mathrm{C}(1)-\mathrm{C}(14)$ & $1.534(5)$ \\
$\mathrm{C}(1)-\mathrm{H}(1)$ & 0.9900 \\
$\mathrm{C}(2)-\mathrm{C}(7)$ & $1.385(6)$ \\
$\mathrm{C}(2)-\mathrm{C}(3)$ & $1.388(5)$ \\
$\mathrm{C}(3)-\mathrm{C}(4)$ & $1.388(6)$ \\
$\mathrm{C}(3)-\mathrm{H}(3)$ & 0.9400 \\
$\mathrm{C}(4)-\mathrm{C}(5)$ & $1.369(6)$ \\
$\mathrm{C}(4)-\mathrm{C}(8)$ & $1.499(6)$ \\
$\mathrm{C}(5)-\mathrm{C}(6)$ & $1.399(6)$ \\
$\mathrm{C}(5)-\mathrm{H}(5)$ & 0.9400 \\
$\mathrm{C}(6)-\mathrm{C}(7)$ & $1.377(6)$ \\
$\mathrm{C}(6)-\mathrm{C}(9)$ & $1.487(7)$ \\
$\mathrm{C}(7)-\mathrm{H}(7)$ & 0.9400 \\
$\mathrm{C}(8)-\mathrm{F}(1)$ & $1.307(6)$ \\
$\mathrm{C}(8)-\mathrm{F}(2)$ & $1.317(7)$ \\
& \\
& \\
&
\end{tabular}




\begin{tabular}{|c|c|}
\hline $\mathrm{C}(8)-\mathrm{F}(3)$ & $1.333(7)$ \\
\hline $\mathrm{C}(9)-\mathrm{F}(4)$ & $1.257(8)$ \\
\hline $\mathrm{C}(9)-\mathrm{F}(5)$ & $1.293(8)$ \\
\hline $\mathrm{C}(9)-\mathrm{F}(6)$ & $1.334(9)$ \\
\hline$C(10)-C(13)$ & $1.495(6)$ \\
\hline$C(10)-C(11)$ & $1.506(8)$ \\
\hline$C(10)-C(12)$ & $1.522(7)$ \\
\hline $\mathrm{C}(11)-\mathrm{H}(11 \mathrm{~A})$ & 0.9700 \\
\hline $\mathrm{C}(11)-\mathrm{H}(11 \mathrm{~B})$ & 0.9700 \\
\hline $\mathrm{C}(11)-\mathrm{H}(11 \mathrm{C})$ & 0.9700 \\
\hline $\mathrm{C}(12)-\mathrm{H}(12 \mathrm{~A})$ & 0.9700 \\
\hline $\mathrm{C}(12)-\mathrm{H}(12 \mathrm{~B})$ & 0.9700 \\
\hline $\mathrm{C}(12)-\mathrm{H}(12 \mathrm{C})$ & 0.9700 \\
\hline $\mathrm{C}(13)-\mathrm{H}(13 \mathrm{~A})$ & 0.9700 \\
\hline $\mathrm{C}(13)-\mathrm{H}(13 \mathrm{~B})$ & 0.9700 \\
\hline $\mathrm{C}(13)-\mathrm{H}(13 \mathrm{C})$ & 0.9700 \\
\hline $\mathrm{C}(14)-\mathrm{H}(14 \mathrm{~A})$ & 0.9800 \\
\hline $\mathrm{C}(14)-\mathrm{H}(14 \mathrm{~B})$ & 0.9800 \\
\hline$C(15)-C(20)$ & $1.386(7)$ \\
\hline$C(15)-C(16)$ & $1.398(8)$ \\
\hline$C(16)-C(17)$ & $1.401(8)$ \\
\hline $\mathrm{C}(16)-\mathrm{H}(16)$ & 0.9400 \\
\hline $\mathrm{C}(17)-\mathrm{C}(18)$ & $1.372(15)$ \\
\hline $\mathrm{C}(17)-\mathrm{H}(17)$ & 0.9400 \\
\hline $\mathrm{C}(18)-\mathrm{C}(19)$ & $1.356(17)$ \\
\hline $\mathrm{C}(18)-\mathrm{H}(18)$ & 0.9400 \\
\hline$C(19)-C(20)$ & $1.369(13)$ \\
\hline C(19)-H(19) & 0.9400 \\
\hline $\mathrm{C}(20)-\mathrm{H}(20)$ & 0.9400 \\
\hline $\mathrm{C}(21)-\mathrm{H}(21 \mathrm{~A})$ & 0.9700 \\
\hline $\mathrm{C}(21)-\mathrm{H}(21 \mathrm{~B})$ & 0.9700 \\
\hline $\mathrm{C}(21)-\mathrm{H}(21 \mathrm{C})$ & 0.9700 \\
\hline $\mathrm{O}(1)-\mathrm{S}(1)-\mathrm{N}(1)$ & $111.0(2)$ \\
\hline $\mathrm{O}(1)-\mathrm{S}(1)-\mathrm{C}(10)$ & $105.9(2)$ \\
\hline $\mathrm{N}(1)-\mathrm{S}(1)-\mathrm{C}(10)$ & $98.82(19)$ \\
\hline $\mathrm{O}(2)-\mathrm{P}(1)-\mathrm{O}(3)$ & $116.2(2)$ \\
\hline $\mathrm{O}(2)-\mathrm{P}(1)-\mathrm{C}(15)$ & $111.6(2)$ \\
\hline
\end{tabular}




\begin{tabular}{|c|c|}
\hline $\mathrm{O}(3)-\mathrm{P}(1)-\mathrm{C}(15)$ & $105.9(3)$ \\
\hline $\mathrm{O}(2)-\mathrm{P}(1)-\mathrm{C}(14)$ & $114.57(18)$ \\
\hline $\mathrm{O}(3)-\mathrm{P}(1)-\mathrm{C}(14)$ & $98.43(18)$ \\
\hline $\mathrm{C}(15)-\mathrm{P}(1)-\mathrm{C}(14)$ & $108.99(19)$ \\
\hline $\mathrm{C}(21)-\mathrm{O}(3)-\mathrm{P}(1)$ & $120.5(4)$ \\
\hline $\mathrm{C}(1)-\mathrm{N}(1)-\mathrm{S}(1)$ & $116.1(2)$ \\
\hline $\mathrm{C}(1)-\mathrm{N}(1)-\mathrm{H}(1 \mathrm{~N})$ & $115(4)$ \\
\hline $\mathrm{S}(1)-\mathrm{N}(1)-\mathrm{H}(1 \mathrm{~N})$ & 112(4) \\
\hline $\mathrm{N}(1)-\mathrm{C}(1)-\mathrm{C}(2)$ & $115.0(3)$ \\
\hline $\mathrm{N}(1)-\mathrm{C}(1)-\mathrm{C}(14)$ & $106.0(3)$ \\
\hline $\mathrm{C}(2)-\mathrm{C}(1)-\mathrm{C}(14)$ & $112.0(3)$ \\
\hline $\mathrm{N}(1)-\mathrm{C}(1)-\mathrm{H}(1)$ & 107.8 \\
\hline $\mathrm{C}(2)-\mathrm{C}(1)-\mathrm{H}(1)$ & 107.8 \\
\hline $\mathrm{C}(14)-\mathrm{C}(1)-\mathrm{H}(1)$ & 107.8 \\
\hline$C(7)-C(2)-C(3)$ & $118.8(3)$ \\
\hline$C(7)-C(2)-C(1)$ & $121.7(3)$ \\
\hline $\mathrm{C}(3)-\mathrm{C}(2)-\mathrm{C}(1)$ & $119.5(3)$ \\
\hline$C(2)-C(3)-C(4)$ & $120.4(4)$ \\
\hline $\mathrm{C}(2)-\mathrm{C}(3)-\mathrm{H}(3)$ & 119.8 \\
\hline $\mathrm{C}(4)-\mathrm{C}(3)-\mathrm{H}(3)$ & 119.8 \\
\hline$C(5)-C(4)-C(3)$ & $121.1(4)$ \\
\hline$C(5)-C(4)-C(8)$ & $120.7(4)$ \\
\hline$C(3)-C(4)-C(8)$ & $118.1(4)$ \\
\hline$C(4)-C(5)-C(6)$ & $118.3(4)$ \\
\hline $\mathrm{C}(4)-\mathrm{C}(5)-\mathrm{H}(5)$ & 120.9 \\
\hline $\mathrm{C}(6)-\mathrm{C}(5)-\mathrm{H}(5)$ & 120.9 \\
\hline$C(7)-C(6)-C(5)$ & $121.0(4)$ \\
\hline$C(7)-C(6)-C(9)$ & $118.9(4)$ \\
\hline$C(5)-C(6)-C(9)$ & $120.1(4)$ \\
\hline$C(6)-C(7)-C(2)$ & $120.4(4)$ \\
\hline $\mathrm{C}(6)-\mathrm{C}(7)-\mathrm{H}(7)$ & 119.8 \\
\hline $\mathrm{C}(2)-\mathrm{C}(7)-\mathrm{H}(7)$ & 119.8 \\
\hline$F(1)-C(8)-F(2)$ & $106.6(5)$ \\
\hline$F(1)-C(8)-F(3)$ & $106.7(5)$ \\
\hline $\mathrm{F}(2)-\mathrm{C}(8)-\mathrm{F}(3)$ & $104.6(5)$ \\
\hline $\mathrm{F}(1)-\mathrm{C}(8)-\mathrm{C}(4)$ & $113.8(5)$ \\
\hline $\mathrm{F}(2)-\mathrm{C}(8)-\mathrm{C}(4)$ & $112.9(4)$ \\
\hline $\mathrm{F}(3)-\mathrm{C}(8)-\mathrm{C}(4)$ & $111.7(4)$ \\
\hline
\end{tabular}




\begin{tabular}{|c|c|}
\hline$F(4)-C(9)-F(5)$ & $113.0(7)$ \\
\hline $\mathrm{F}(4)-\mathrm{C}(9)-\mathrm{F}(6)$ & $102.8(7)$ \\
\hline$F(5)-C(9)-F(6)$ & $98.7(6)$ \\
\hline $\mathrm{F}(4)-\mathrm{C}(9)-\mathrm{C}(6)$ & $115.6(5)$ \\
\hline $\mathrm{F}(5)-\mathrm{C}(9)-\mathrm{C}(6)$ & $114.1(5)$ \\
\hline $\mathrm{F}(6)-\mathrm{C}(9)-\mathrm{C}(6)$ & $110.5(6)$ \\
\hline$C(13)-C(10)-C(11)$ & $111.9(5)$ \\
\hline$C(13)-C(10)-C(12)$ & $111.5(5)$ \\
\hline$C(11)-C(10)-C(12)$ & $110.9(6)$ \\
\hline$C(13)-C(10)-S(1)$ & $109.7(3)$ \\
\hline$C(11)-C(10)-S(1)$ & $108.9(3)$ \\
\hline$C(12)-C(10)-S(1)$ & $103.6(4)$ \\
\hline $\mathrm{C}(10)-\mathrm{C}(11)-\mathrm{H}(11 \mathrm{~A})$ & 109.5 \\
\hline $\mathrm{C}(10)-\mathrm{C}(11)-\mathrm{H}(11 \mathrm{~B})$ & 109.5 \\
\hline $\mathrm{H}(11 \mathrm{~A})-\mathrm{C}(11)-\mathrm{H}(11 \mathrm{~B})$ & 109.5 \\
\hline $\mathrm{C}(10)-\mathrm{C}(11)-\mathrm{H}(11 \mathrm{C})$ & 109.5 \\
\hline $\mathrm{H}(11 \mathrm{~A})-\mathrm{C}(11)-\mathrm{H}(11 \mathrm{C})$ & 109.5 \\
\hline $\mathrm{H}(11 \mathrm{~B})-\mathrm{C}(11)-\mathrm{H}(11 \mathrm{C})$ & 109.5 \\
\hline $\mathrm{C}(10)-\mathrm{C}(12)-\mathrm{H}(12 \mathrm{~A})$ & 109.5 \\
\hline $\mathrm{C}(10)-\mathrm{C}(12)-\mathrm{H}(12 \mathrm{~B})$ & 109.5 \\
\hline $\mathrm{H}(12 \mathrm{~A})-\mathrm{C}(12)-\mathrm{H}(12 \mathrm{~B})$ & 109.5 \\
\hline $\mathrm{C}(10)-\mathrm{C}(12)-\mathrm{H}(12 \mathrm{C})$ & 109.5 \\
\hline $\mathrm{H}(12 \mathrm{~A})-\mathrm{C}(12)-\mathrm{H}(12 \mathrm{C})$ & 109.5 \\
\hline $\mathrm{H}(12 \mathrm{~B})-\mathrm{C}(12)-\mathrm{H}(12 \mathrm{C})$ & 109.5 \\
\hline $\mathrm{C}(10)-\mathrm{C}(13)-\mathrm{H}(13 \mathrm{~A})$ & 109.5 \\
\hline $\mathrm{C}(10)-\mathrm{C}(13)-\mathrm{H}(13 \mathrm{~B})$ & 109.5 \\
\hline $\mathrm{H}(13 \mathrm{~A})-\mathrm{C}(13)-\mathrm{H}(13 \mathrm{~B})$ & 109.5 \\
\hline $\mathrm{C}(10)-\mathrm{C}(13)-\mathrm{H}(13 \mathrm{C})$ & 109.5 \\
\hline $\mathrm{H}(13 \mathrm{~A})-\mathrm{C}(13)-\mathrm{H}(13 \mathrm{C})$ & 109.5 \\
\hline $\mathrm{H}(13 \mathrm{~B})-\mathrm{C}(13)-\mathrm{H}(13 \mathrm{C})$ & 109.5 \\
\hline $\mathrm{C}(1)-\mathrm{C}(14)-\mathrm{P}(1)$ & $115.4(3)$ \\
\hline $\mathrm{C}(1)-\mathrm{C}(14)-\mathrm{H}(14 \mathrm{~A})$ & 108.4 \\
\hline $\mathrm{P}(1)-\mathrm{C}(14)-\mathrm{H}(14 \mathrm{~A})$ & 108.4 \\
\hline $\mathrm{C}(1)-\mathrm{C}(14)-\mathrm{H}(14 \mathrm{~B})$ & 108.4 \\
\hline$P(1)-C(14)-H(14 B)$ & 108.4 \\
\hline $\mathrm{H}(14 \mathrm{~A})-\mathrm{C}(14)-\mathrm{H}(14 \mathrm{~B})$ & 107.5 \\
\hline$C(20)-C(15)-C(16)$ & $119.4(5)$ \\
\hline $\mathrm{C}(20)-\mathrm{C}(15)-\mathrm{P}(1)$ & $120.4(5)$ \\
\hline
\end{tabular}




$\begin{array}{ll}\mathrm{C}(16)-\mathrm{C}(15)-\mathrm{P}(1) & 119.9(4) \\ \mathrm{C}(15)-\mathrm{C}(16)-\mathrm{C}(17) & 119.4(6) \\ \mathrm{C}(15)-\mathrm{C}(16)-\mathrm{H}(16) & 120.3 \\ \mathrm{C}(17)-\mathrm{C}(16)-\mathrm{H}(16) & 120.3 \\ \mathrm{C}(18)-\mathrm{C}(17)-\mathrm{C}(16) & 118.8(9) \\ \mathrm{C}(18)-\mathrm{C}(17)-\mathrm{H}(17) & 120.6 \\ \mathrm{C}(16)-\mathrm{C}(17)-\mathrm{H}(17) & 120.6 \\ \mathrm{C}(19)-\mathrm{C}(18)-\mathrm{C}(17) & 121.9(7) \\ \mathrm{C}(19)-\mathrm{C}(18)-\mathrm{H}(18) & 119.0 \\ \mathrm{C}(17)-\mathrm{C}(18)-\mathrm{H}(18) & 119.0 \\ \mathrm{C}(18)-\mathrm{C}(19)-\mathrm{C}(20) & 120.0(8) \\ \mathrm{C}(18)-\mathrm{C}(19)-\mathrm{H}(19) & 120.0 \\ \mathrm{C}(20)-\mathrm{C}(19)-\mathrm{H}(19) & 120.0 \\ \mathrm{C}(19)-\mathrm{C}(20)-\mathrm{C}(15) & 120.4(8) \\ \mathrm{C}(19)-\mathrm{C}(20)-\mathrm{H}(20) & 119.8 \\ \mathrm{C}(15)-\mathrm{C}(20)-\mathrm{H}(20) & 119.8 \\ \mathrm{O}(3)-\mathrm{C}(21)-\mathrm{H}(21 \mathrm{~A}) & 109.5 \\ \mathrm{O}(3)-\mathrm{C}(21)-\mathrm{H}(21 \mathrm{~B}) & 109.5 \\ \mathrm{H}(21 \mathrm{~A})-\mathrm{C}(21)-\mathrm{H}(21 \mathrm{~B}) & 109.5 \\ \mathrm{O}(3)-\mathrm{C}(21)-\mathrm{H}(21 \mathrm{C}) & 109.5 \\ \mathrm{H}(21 \mathrm{~A})-\mathrm{C}(21)-\mathrm{H}(21 \mathrm{C}) & 109.5 \\ \mathrm{H}(21 \mathrm{~B})-\mathrm{C}(21)-\mathrm{H}(21 \mathrm{C}) & 109.5 \\ & \\ & \end{array}$

Symmetry transformations used to generate equivalent atoms:

Table 4. Anisotropic displacement parameters $\left(\AA^{2} \times 10^{3}\right)$ for compound $\mathbf{9}(\boldsymbol{R}, \boldsymbol{S}, \boldsymbol{S})$. The anisotropic displacement factor exponent takes the form: $-2 \pi^{2}\left[\mathrm{~h}^{2} \mathrm{a}^{* 2} \mathrm{U}^{11}+\ldots+2 \mathrm{~h} \mathrm{k} \mathrm{a}^{*}\right.$ $b^{*} \mathrm{U}^{12}$ ]

\begin{tabular}{lcccccc}
\hline & $\mathrm{U}^{11}$ & $\mathrm{U}^{22}$ & $\mathrm{U}^{33}$ & $\mathrm{U}^{23}$ & $\mathrm{U}^{13}$ & $\mathrm{U}^{12}$ \\
\hline $\mathrm{S}(1)$ & $39(1)$ & $30(1)$ & $44(1)$ & $1(1)$ & $-15(1)$ & $-2(1)$ \\
$\mathrm{P}(1)$ & $27(1)$ & $36(1)$ & $37(1)$ & $-7(1)$ & $-2(1)$ & $8(1)$ \\
$\mathrm{O}(1)$ & $101(3)$ & $49(2)$ & $35(2)$ & $-12(2)$ & $-14(2)$ & $12(2)$ \\
$\mathrm{O}(2)$ & $25(1)$ & $47(2)$ & $45(2)$ & $1(1)$ & $5(1)$ & $7(1)$ \\
$\mathrm{O}(3)$ & $74(3)$ & $57(2)$ & $56(2)$ & $-26(2)$ & $-24(2)$ & $32(2)$ \\
$\mathrm{N}(1)$ & $25(2)$ & $30(2)$ & $31(2)$ & $1(1)$ & $-4(1)$ & $3(1)$ \\
$\mathrm{C}(1)$ & $24(2)$ & $30(2)$ & $25(2)$ & $3(1)$ & $-1(1)$ & $3(1)$
\end{tabular}




\begin{tabular}{|c|c|c|c|c|c|c|}
\hline $\mathrm{C}(2)$ & $31(2)$ & $27(2)$ & $27(2)$ & $-1(1)$ & $-3(1)$ & 1(1) \\
\hline $\mathrm{C}(3)$ & $28(2)$ & $32(2)$ & $29(2)$ & $-1(2)$ & $0(2)$ & $3(2)$ \\
\hline $\mathrm{C}(4)$ & $35(2)$ & $39(2)$ & $32(2)$ & $1(2)$ & $-5(2)$ & $8(2)$ \\
\hline $\mathrm{C}(5)$ & $48(2)$ & $48(2)$ & $30(2)$ & 10(2) & $-1(2)$ & $4(2)$ \\
\hline$C(6)$ & $34(2)$ & $48(2)$ & $37(2)$ & $5(2)$ & $6(2)$ & 2(2) \\
\hline $\mathrm{C}(7)$ & $27(2)$ & 41(2) & $35(2)$ & $3(2)$ & $0(2)$ & $4(2)$ \\
\hline $\mathrm{C}(8)$ & $52(3)$ & $59(3)$ & $39(2)$ & $4(2)$ & $-16(2)$ & $9(2)$ \\
\hline $\mathrm{F}(1)$ & $61(2)$ & $112(3)$ & $46(2)$ & $22(2)$ & $-14(1)$ & $-1(2)$ \\
\hline $\mathrm{F}(2)$ & $48(2)$ & $120(3)$ & $98(3)$ & $51(2)$ & $-31(2)$ & $-18(2)$ \\
\hline $\mathrm{F}(3)$ & $93(3)$ & $114(3)$ & $103(3)$ & $-21(2)$ & $-44(2)$ & $57(2)$ \\
\hline $\mathrm{C}(9)$ & $41(3)$ & $102(4)$ & $47(3)$ & 19(3) & $11(2)$ & $6(3)$ \\
\hline $\mathrm{F}(4)$ & $83(3)$ & $265(5)$ & $130(4)$ & $129(4)$ & $28(3)$ & $3(3)$ \\
\hline $\mathrm{F}(5)$ & $63(2)$ & $193(5)$ & $110(3)$ & 12(3) & $18(2)$ & $-37(3)$ \\
\hline $\mathrm{F}(6)$ & $117(4)$ & $167(4)$ & $175(5)$ & $-11(4)$ & $94(3)$ & $20(3)$ \\
\hline$C(10)$ & $33(2)$ & $33(2)$ & $52(3)$ & $12(2)$ & $-1(2)$ & $1(2)$ \\
\hline $\mathrm{C}(11)$ & $72(4)$ & $104(5)$ & 64(4) & $46(3)$ & $24(3)$ & $39(4)$ \\
\hline $\mathrm{C}(12)$ & $131(7)$ & $32(3)$ & $153(7)$ & $24(3)$ & $-69(6)$ & $-17(3)$ \\
\hline $\mathrm{C}(13)$ & $36(2)$ & $60(3)$ & 70(4) & 11(3) & $0(2)$ & $12(2)$ \\
\hline $\mathrm{C}(14)$ & $30(2)$ & $35(2)$ & $30(2)$ & $-5(2)$ & $-5(2)$ & $9(2)$ \\
\hline $\mathrm{C}(15)$ & $28(2)$ & $28(2)$ & 77(3) & $4(2)$ & $6(2)$ & $2(2)$ \\
\hline $\mathrm{C}(16)$ & $55(3)$ & $35(2)$ & $60(3)$ & $6(2)$ & $13(2)$ & $8(2)$ \\
\hline $\mathrm{C}(17)$ & $108(5)$ & $59(4)$ & $107(5)$ & $39(4)$ & $42(4)$ & $28(4)$ \\
\hline $\mathrm{C}(18)$ & $98(6)$ & $76(5)$ & 192(9) & $65(6)$ & $69(6)$ & 1(4) \\
\hline$C(19)$ & $53(4)$ & $93(6)$ & 271(12) & $80(8)$ & $11(5)$ & $-18(4)$ \\
\hline $\mathrm{C}(20)$ & $38(3)$ & $53(3)$ & $162(7)$ & $35(4)$ & $-16(3)$ & $-10(2)$ \\
\hline $\mathrm{C}(21)$ & $108(5)$ & $70(4)$ & 68(4) & $-39(3)$ & $-25(4)$ & $45(4)$ \\
\hline
\end{tabular}

Table 5. Hydrogen coordinates $\left(\times 10^{4}\right)$ and isotropic displacement parameters $\left(\AA^{2} \times 10^{3}\right)$ for compound $\mathbf{9}(\boldsymbol{R}, S, S)$.

$\mathrm{X}$

$\begin{array}{llll}\text { y } & \text { z } & \text { U(eq) }\end{array}$

\begin{tabular}{lllll}
\hline $\mathrm{H}(1 \mathrm{~N})$ & $3870(20)$ & $9250(40)$ & $5080(30)$ & 43 \\
$\mathrm{H}(1)$ & 6252 & 8660 & 5040 & 31 \\
$\mathrm{H}(3)$ & 7232 & 8088 & 6141 & 35 \\
$\mathrm{H}(5)$ & 5438 & 7006 & 7997 & 50
\end{tabular}




\begin{tabular}{lrrrr}
$\mathrm{H}(7)$ & 3547 & 8027 & 6148 & 41 \\
$\mathrm{H}(11 \mathrm{~A})$ & 4446 & 10450 & 3753 & 120 \\
$\mathrm{H}(11 \mathrm{~B})$ & 5665 & 11053 & 3934 & 120 \\
$\mathrm{H}(11 \mathrm{C})$ & 4515 & 11620 & 3586 & 120 \\
$\mathrm{H}(12 \mathrm{~A})$ & 4355 & 12848 & 4702 & 158 \\
$\mathrm{H}(12 \mathrm{~B})$ & 5629 & 12380 & 4954 & 158 \\
$\mathrm{H}(12 \mathrm{C})$ & 4527 & 12418 & 5549 & 158 \\
$\mathrm{H}(13 \mathrm{~A})$ & 2720 & 11186 & 5333 & 83 \\
$\mathrm{H}(13 \mathrm{~B})$ & 2706 & 10574 & 4548 & 83 \\
$\mathrm{H}(13 \mathrm{C})$ & 2569 & 11760 & 4537 & 83 \\
$\mathrm{H}(14 \mathrm{~A})$ & 5073 & 7920 & 4074 & 38 \\
$\mathrm{H}(14 \mathrm{~B})$ & 4113 & 7523 & 4687 & 38 \\
$\mathrm{H}(16)$ & 6451 & 5690 & 6144 & 60 \\
$\mathrm{H}(17)$ & 5385 & 4769 & 7091 & 110 \\
$\mathrm{H}(18)$ & 3388 & 4288 & 6854 & 146 \\
$\mathrm{H}(19)$ & 2460 & 4617 & 5699 & 167 \\
$\mathrm{H}(20)$ & 3482 & 5512 & 4756 & 101 \\
$\mathrm{H}(21 \mathrm{~A})$ & 6812 & 5046 & 3786 & 123 \\
$\mathrm{H}(21 \mathrm{~B})$ & 6027 & 5118 & 3017 & 123 \\
$\mathrm{H}(21 \mathrm{C})$ & 5489 & 4557 & 3751 & 123 \\
\hline & & & & \\
\hline
\end{tabular}

Table 6. Torsion angles $\left[{ }^{\circ}\right]$ for compound $\mathbf{9}(\boldsymbol{R}, S, S)$.

\begin{tabular}{lc}
\hline $\mathrm{O}(2)-\mathrm{P}(1)-\mathrm{O}(3)-\mathrm{C}(21)$ & $52.2(6)$ \\
$\mathrm{C}(15)-\mathrm{P}(1)-\mathrm{O}(3)-\mathrm{C}(21)$ & $-72.4(6)$ \\
$\mathrm{C}(14)-\mathrm{P}(1)-\mathrm{O}(3)-\mathrm{C}(21)$ & $175.0(5)$ \\
$\mathrm{O}(1)-\mathrm{S}(1)-\mathrm{N}(1)-\mathrm{C}(1)$ & $-94.0(3)$ \\
$\mathrm{C}(10)-\mathrm{S}(1)-\mathrm{N}(1)-\mathrm{C}(1)$ & $155.1(3)$ \\
$\mathrm{S}(1)-\mathrm{N}(1)-\mathrm{C}(1)-\mathrm{C}(2)$ & $69.0(4)$ \\
$\mathrm{S}(1)-\mathrm{N}(1)-\mathrm{C}(1)-\mathrm{C}(14)$ & $-166.6(2)$ \\
$\mathrm{N}(1)-\mathrm{C}(1)-\mathrm{C}(2)-\mathrm{C}(7)$ & $51.7(5)$ \\
$\mathrm{C}(14)-\mathrm{C}(1)-\mathrm{C}(2)-\mathrm{C}(7)$ & $-69.4(5)$ \\
$\mathrm{N}(1)-\mathrm{C}(1)-\mathrm{C}(2)-\mathrm{C}(3)$ & $-130.3(4)$ \\
$\mathrm{C}(14)-\mathrm{C}(1)-\mathrm{C}(2)-\mathrm{C}(3)$ & $108.6(4)$ \\
$\mathrm{C}(7)-\mathrm{C}(2)-\mathrm{C}(3)-\mathrm{C}(4)$ & $2.6(6)$ \\
$\mathrm{C}(1)-\mathrm{C}(2)-\mathrm{C}(3)-\mathrm{C}(4)$ & $-175.6(3)$ \\
$\mathrm{C}(2)-\mathrm{C}(3)-\mathrm{C}(4)-\mathrm{C}(5)$ & $-0.8(6)$
\end{tabular}




\begin{tabular}{|c|c|}
\hline $\mathrm{C}(2)-\mathrm{C}(3)-\mathrm{C}(4)-\mathrm{C}(8)$ & $176.0(4)$ \\
\hline$C(3)-C(4)-C(5)-C(6)$ & $-1.4(7)$ \\
\hline$C(8)-C(4)-C(5)-C(6)$ & $-178.2(5)$ \\
\hline$C(4)-C(5)-C(6)-C(7)$ & $1.9(7)$ \\
\hline$C(4)-C(5)-C(6)-C(9)$ & $-177.4(5)$ \\
\hline$C(5)-C(6)-C(7)-C(2)$ & $-0.2(7)$ \\
\hline$C(9)-C(6)-C(7)-C(2)$ & $179.1(5)$ \\
\hline$C(3)-C(2)-C(7)-C(6)$ & $-2.1(6)$ \\
\hline$C(1)-C(2)-C(7)-C(6)$ & $176.0(4)$ \\
\hline$C(5)-C(4)-C(8)-F(1)$ & $-22.0(7)$ \\
\hline $\mathrm{C}(3)-\mathrm{C}(4)-\mathrm{C}(8)-\mathrm{F}(1)$ & $161.2(4)$ \\
\hline $\mathrm{C}(5)-\mathrm{C}(4)-\mathrm{C}(8)-\mathrm{F}(2)$ & $-143.7(5)$ \\
\hline $\mathrm{C}(3)-\mathrm{C}(4)-\mathrm{C}(8)-\mathrm{F}(2)$ & $39.5(6)$ \\
\hline $\mathrm{C}(5)-\mathrm{C}(4)-\mathrm{C}(8)-\mathrm{F}(3)$ & $98.8(6)$ \\
\hline $\mathrm{C}(3)-\mathrm{C}(4)-\mathrm{C}(8)-\mathrm{F}(3)$ & $-78.0(6)$ \\
\hline$C(7)-C(6)-C(9)-F(4)$ & $171.8(7)$ \\
\hline$C(5)-C(6)-C(9)-F(4)$ & $-8.9(10)$ \\
\hline$C(7)-C(6)-C(9)-F(5)$ & $38.2(8)$ \\
\hline$C(5)-C(6)-C(9)-F(5)$ & $-142.5(6)$ \\
\hline$C(7)-C(6)-C(9)-F(6)$ & $-72.0(7)$ \\
\hline $\mathrm{C}(5)-\mathrm{C}(6)-\mathrm{C}(9)-\mathrm{F}(6)$ & $107.3(6)$ \\
\hline $\mathrm{O}(1)-\mathrm{S}(1)-\mathrm{C}(10)-\mathrm{C}(13)$ & $-50.1(4)$ \\
\hline $\mathrm{N}(1)-\mathrm{S}(1)-\mathrm{C}(10)-\mathrm{C}(13)$ & $64.8(4)$ \\
\hline $\mathrm{O}(1)-\mathrm{S}(1)-\mathrm{C}(10)-\mathrm{C}(11)$ & $-172.9(4)$ \\
\hline $\mathrm{N}(1)-\mathrm{S}(1)-\mathrm{C}(10)-\mathrm{C}(11)$ & $-58.0(5)$ \\
\hline $\mathrm{O}(1)-\mathrm{S}(1)-\mathrm{C}(10)-\mathrm{C}(12)$ & $69.0(5)$ \\
\hline $\mathrm{N}(1)-\mathrm{S}(1)-\mathrm{C}(10)-\mathrm{C}(12)$ & $-176.1(5)$ \\
\hline $\mathrm{N}(1)-\mathrm{C}(1)-\mathrm{C}(14)-\mathrm{P}(1)$ & $180.0(3)$ \\
\hline $\mathrm{C}(2)-\mathrm{C}(1)-\mathrm{C}(14)-\mathrm{P}(1)$ & $-53.8(4)$ \\
\hline $\mathrm{O}(2)-\mathrm{P}(1)-\mathrm{C}(14)-\mathrm{C}(1)$ & $-37.1(3)$ \\
\hline $\mathrm{O}(3)-\mathrm{P}(1)-\mathrm{C}(14)-\mathrm{C}(1)$ & $-161.1(3)$ \\
\hline $\mathrm{C}(15)-\mathrm{P}(1)-\mathrm{C}(14)-\mathrm{C}(1)$ & $88.8(3)$ \\
\hline $\mathrm{O}(2)-\mathrm{P}(1)-\mathrm{C}(15)-\mathrm{C}(20)$ & $-169.5(5)$ \\
\hline $\mathrm{O}(3)-\mathrm{P}(1)-\mathrm{C}(15)-\mathrm{C}(20)$ & $-42.1(5)$ \\
\hline $\mathrm{C}(14)-\mathrm{P}(1)-\mathrm{C}(15)-\mathrm{C}(20)$ & $62.9(5)$ \\
\hline $\mathrm{O}(2)-\mathrm{P}(1)-\mathrm{C}(15)-\mathrm{C}(16)$ & $16.7(4)$ \\
\hline $\mathrm{O}(3)-\mathrm{P}(1)-\mathrm{C}(15)-\mathrm{C}(16)$ & $144.1(4)$ \\
\hline $\mathrm{C}(14)-\mathrm{P}(1)-\mathrm{C}(15)-\mathrm{C}(16)$ & $-110.9(4)$ \\
\hline
\end{tabular}




$\begin{array}{lc}\mathrm{C}(20)-\mathrm{C}(15)-\mathrm{C}(16)-\mathrm{C}(17) & 0.2(8) \\ \mathrm{P}(1)-\mathrm{C}(15)-\mathrm{C}(16)-\mathrm{C}(17) & 174.0(4) \\ \mathrm{C}(15)-\mathrm{C}(16)-\mathrm{C}(17)-\mathrm{C}(18) & -0.9(10) \\ \mathrm{C}(16)-\mathrm{C}(17)-\mathrm{C}(18)-\mathrm{C}(19) & 1.6(14) \\ \mathrm{C}(17)-\mathrm{C}(18)-\mathrm{C}(19)-\mathrm{C}(20) & -1.4(17) \\ \mathrm{C}(18)-\mathrm{C}(19)-\mathrm{C}(20)-\mathrm{C}(15) & 0.6(16) \\ \mathrm{C}(16)-\mathrm{C}(15)-\mathrm{C}(20)-\mathrm{C}(19) & 0.0(11) \\ \mathrm{P}(1)-\mathrm{C}(15)-\mathrm{C}(20)-\mathrm{C}(19) & -173.8(7)\end{array}$

Symmetry transformations used to generate equivalent atoms:

Table 7. Hydrogen bonds for compound $\mathbf{9}(\boldsymbol{R}, S, S)\left[\AA\right.$ and $\left.^{\circ}\right]$.

\begin{tabular}{lllll}
\hline $\mathrm{D}-\mathrm{H} \ldots \mathrm{A}$ & $\mathrm{d}(\mathrm{D}-\mathrm{H})$ & $\mathrm{d}(\mathrm{H} \ldots \mathrm{A})$ & $\mathrm{d}(\mathrm{D} \ldots \mathrm{A})$ & $<(\mathrm{DHA})$ \\
\hline $\mathrm{N}(1)-\mathrm{H}(1 \mathrm{~N}) \ldots \mathrm{O}(2) \# 1$ & $0.83(2)$ & $2.21(3)$ & $2.999(4)$ & $159(5)$ \\
& & & & \\
\hline
\end{tabular}

Symmetry transformations used to generate equivalent atoms:

\#1 x-1/2,-y+3/2,-z+1

\section{Methyl 2-(tert-butylsulfinamide)-2-[3,5-bis(trifluoromethyl)phenyl]ethyl}

phenylphosphinate, $9(R, S, R)$.

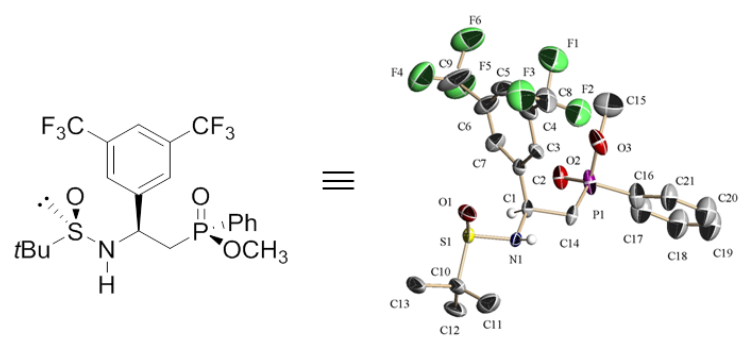

Table 1. Crystal data and structure refinement for compound $\mathbf{9}(\boldsymbol{R}, \boldsymbol{S}, \boldsymbol{R})$.

Empirical formula

Formula weight

Temperature

Wavelength

Crystal system

Space group

Unit cell dimensions
$\mathrm{C}_{21} \mathrm{H}_{24} \mathrm{~F}_{6} \mathrm{NO}_{3} \mathrm{PS}$

515.44

213(2) K

$0.71073 \AA$

Orthorhombic

P $22_{1} 2$

$\mathrm{a}=10.3003(6) \AA \quad \alpha=90^{\circ}$. 
Volume

Z

Density (calculated)

Absorption coefficient

$\mathrm{F}(000)$

Crystal size

Theta range for data collection

Index ranges

Reflections collected

Independent reflections

Completeness to theta $=25.25^{\circ}$

Absorption correction

Max. and min. transmission

Refinement method

Data / restraints / parameters

Goodness-of-fit on $\mathrm{F}^{2}$

Final $\mathrm{R}$ indices $[\mathrm{I}>2 \operatorname{sigma}(\mathrm{I})]$

$\mathrm{R}$ indices (all data)

Absolute structure parameter

Largest diff. peak and hole

$$
\begin{array}{ll}
\mathrm{b}=10.6894(4) \AA & \beta=90^{\circ} . \\
\mathrm{c}=22.8824(13) \AA & \gamma=90^{\circ} .
\end{array}
$$

$$
\text { 2519.4(2) } \AA^{3}
$$

4

$1.359 \mathrm{Mg} / \mathrm{m}^{3}$

$0.258 \mathrm{~mm}^{-1}$

1064

$0.50 \times 0.45 \times 0.40 \mathrm{~mm}^{3}$

1.78 to $25.25^{\circ}$.

$-12<=\mathrm{h}<=9,-11<=\mathrm{k}<=11,-27<=\mathrm{l}<=20$

23443

$4539[\mathrm{R}(\mathrm{int})=0.0418]$

$99.4 \%$

Semi-empirical from equivalents

0.9039 and 0.8819

Full-matrix least-squares on $\mathrm{F}^{2}$

4539 / $121 / 293$

1.094

$\mathrm{R} 1=0.0607, \mathrm{wR} 2=0.1370$

$\mathrm{R} 1=0.0889, \mathrm{wR} 2=0.2106$

$0.03(7)$

\begin{tabular}{|c|c|c|c|c|}
\hline & $\mathrm{x}$ & $\mathrm{y}$ & $\mathrm{z}$ & $\mathrm{U}(\mathrm{eq})$ \\
\hline$S(1)$ & $4700(2)$ & $2913(2)$ & $8374(1)$ & $45(1)$ \\
\hline $\mathrm{P}(1)$ & $4326(2)$ & 1996(3) & 10691(1) & $62(1)$ \\
\hline $\mathrm{O}(1)$ & $5665(7)$ & $2057(5)$ & $8094(2)$ & $68(2)$ \\
\hline $\mathrm{O}(2)$ & 2902(4) & $1910(7)$ & $10618(2)$ & $64(2)$ \\
\hline $\mathrm{O}(3)$ & $5085(6)$ & $668(8)$ & $10673(3)$ & $83(2)$ \\
\hline $\mathrm{N}(1)$ & $5173(5)$ & $3237(5)$ & $9054(2)$ & $38(1)$ \\
\hline$C(1)$ & $4539(6)$ & $2435(6)$ & $9498(2)$ & $40(1)$ \\
\hline $\mathrm{C}(2)$ & $4663(7)$ & $1068(7)$ & $9377(2)$ & $42(1)$ \\
\hline
\end{tabular}

1.061 and -0.666 e. $\AA^{-3}$

Table 2. Atomic coordinates $\left(\times 10^{4}\right)$ and equivalent isotropic displacement parameters $\left(\AA^{2} \times 10^{3}\right)$ for compound $\mathbf{9}(\boldsymbol{R}, S, \boldsymbol{R})$. U(eq) is defined as one third of the trace of the orthogonalized $\mathrm{Uij}$ tensor. 


\begin{tabular}{|c|c|c|c|c|}
\hline $\mathrm{C}(3)$ & $5856(8)$ & $471(8)$ & $9339(3)$ & $52(2)$ \\
\hline $\mathrm{C}(4)$ & $5954(14)$ & $-833(10)$ & $9232(4)$ & $85(2)$ \\
\hline$C(5)$ & $4818(17)$ & $-1508(10)$ & $9172(5)$ & 103(3) \\
\hline$C(6)$ & $3647(14)$ & $-897(12)$ & $9205(5)$ & $97(3)$ \\
\hline$C(7)$ & $3574(9)$ & $335(9)$ & $9327(4)$ & $67(2)$ \\
\hline $\mathrm{C}(8)$ & $7340(20)$ & $-1303(16)$ & $9146(8)$ & $138(4)$ \\
\hline $\mathrm{F}(1)$ & $7242(13)$ & $-2526(11)$ & $9361(7)$ & $177(4)$ \\
\hline $\mathrm{F}(2)$ & $8148(10)$ & $-861(10)$ & $9444(5)$ & $146(3)$ \\
\hline $\mathrm{F}(3)$ & $7564(12)$ & $-1623(12)$ & $8639(5)$ & $165(3)$ \\
\hline$C(9)$ & $2340(20)$ & $-1649(17)$ & $9073(9)$ & $161(5)$ \\
\hline $\mathrm{F}(4)$ & $2205(12)$ & $-1913(13)$ & $8541(6)$ & $176(4)$ \\
\hline $\mathrm{F}(5)$ & $1460(11)$ & $-1301(12)$ & $9430(6)$ & $163(4)$ \\
\hline$F(6)$ & $2593(13)$ & $-2873(11)$ & $9294(6)$ & $170(3)$ \\
\hline$C(10)$ & $4916(11)$ & $4409(9)$ & $8031(4)$ & $72(2)$ \\
\hline$C(11)$ & $6265(13)$ & $4901(12)$ & $8145(6)$ & $102(4)$ \\
\hline$C(12)$ & $3861(14)$ & $5277(10)$ & $8282(5)$ & $96(3)$ \\
\hline$C(13)$ & 4701(17) & $4170(9)$ & $7377(4)$ & $105(4)$ \\
\hline$C(14)$ & $5089(6)$ & $2810(8)$ & $10098(3)$ & $48(2)$ \\
\hline$C(15)$ & $4765(16)$ & $-389(18)$ & 11003(9) & $134(5)$ \\
\hline$C(16)$ & $4767(6)$ & $2621(8)$ & $11364(2)$ & $78(2)$ \\
\hline$C(17)$ & $3831(5)$ & $3214(10)$ & $11702(3)$ & $98(3)$ \\
\hline $\mathrm{C}(18)$ & $4186(8)$ & $3819(12)$ & $12215(4)$ & $127(4)$ \\
\hline$C(19)$ & $5479(9)$ & $3831(12)$ & $12390(4)$ & $134(4)$ \\
\hline$C(20)$ & $6415(6)$ & $3237(11)$ & $12052(4)$ & $128(4)$ \\
\hline $\mathrm{C}(21)$ & $6059(5)$ & $2632(9)$ & 11539(3) & $87(3)$ \\
\hline
\end{tabular}

Table 3. Bond lengths $[\AA]$ and angles $\left[^{\circ}\right]$ for compound $\mathbf{9}(\boldsymbol{R}, \boldsymbol{S}, \boldsymbol{R})$.

\begin{tabular}{llll}
\hline $\mathrm{S}(1)-\mathrm{O}(1)$ & $1.495(6)$ & $\mathrm{N}(1)-\mathrm{H}(1 \mathrm{~N})$ & $0.84(2)$ \\
$\mathrm{S}(1)-\mathrm{N}(1)$ & $1.668(5)$ & $\mathrm{C}(1)-\mathrm{C}(2)$ & $1.493(11)$ \\
$\mathrm{S}(1)-\mathrm{C}(10)$ & $1.795(9)$ & $\mathrm{C}(1)-\mathrm{C}(14)$ & $1.538(8)$ \\
$\mathrm{P}(1)-\mathrm{O}(2)$ & $1.480(5)$ & $\mathrm{C}(1)-\mathrm{H}(1)$ & 0.9900 \\
$\mathrm{P}(1)-\mathrm{O}(3)$ & $1.621(8)$ & $\mathrm{C}(2)-\mathrm{C}(7)$ & $1.373(11)$ \\
$\mathrm{P}(1)-\mathrm{C}(16)$ & $1.739(5)$ & $\mathrm{C}(2)-\mathrm{C}(3)$ & $1.388(10)$ \\
$\mathrm{P}(1)-\mathrm{C}(14)$ & $1.792(7)$ & $\mathrm{C}(3)-\mathrm{C}(4)$ & $1.419(14)$ \\
$\mathrm{O}(3)-\mathrm{C}(15)$ & $1.397(19)$ & $\mathrm{C}(3)-\mathrm{H}(3)$ & 0.9400 \\
$\mathrm{~N}(1)-\mathrm{C}(1)$ & $1.480(8)$ & $\mathrm{C}(4)-\mathrm{C}(5)$ & $1.382(19)$
\end{tabular}




\begin{tabular}{|c|c|c|c|}
\hline $\mathrm{C}(4)-\mathrm{C}(8)$ & $1.53(2)$ & $\mathrm{C}(20)-\mathrm{H}(20)$ & 0.9400 \\
\hline$C(5)-C(6)$ & $1.37(2)$ & $\mathrm{C}(21)-\mathrm{H}(21)$ & 0.9400 \\
\hline $\mathrm{C}(5)-\mathrm{H}(5)$ & 0.9400 & & \\
\hline$C(6)-C(7)$ & $1.348(16)$ & $\mathrm{O}(1)-\mathrm{S}(1)-\mathrm{N}(1)$ & $109.4(3)$ \\
\hline $\mathrm{C}(6)-\mathrm{C}(9)$ & $1.59(2)$ & $\mathrm{O}(1)-\mathrm{S}(1)-\mathrm{C}(10)$ & $106.0(4)$ \\
\hline $\mathrm{C}(7)-\mathrm{H}(7)$ & 0.9400 & $\mathrm{~N}(1)-\mathrm{S}(1)-\mathrm{C}(10)$ & $100.8(3)$ \\
\hline $\mathrm{C}(8)-\mathrm{F}(2)$ & $1.175(18)$ & $\mathrm{O}(2)-\mathrm{P}(1)-\mathrm{O}(3)$ & $114.9(4)$ \\
\hline $\mathrm{C}(8)-\mathrm{F}(3)$ & $1.231(19)$ & $\mathrm{O}(2)-\mathrm{P}(1)-\mathrm{C}(16)$ & $112.5(3)$ \\
\hline $\mathrm{C}(8)-\mathrm{F}(1)$ & $1.40(2)$ & $\mathrm{O}(3)-\mathrm{P}(1)-\mathrm{C}(16)$ & $103.4(4)$ \\
\hline $\mathrm{C}(9)-\mathrm{F}(4)$ & $1.26(2)$ & $\mathrm{O}(2)-\mathrm{P}(1)-\mathrm{C}(14)$ & $112.3(3)$ \\
\hline $\mathrm{C}(9)-\mathrm{F}(5)$ & $1.28(2)$ & $\mathrm{O}(3)-\mathrm{P}(1)-\mathrm{C}(14)$ & 101.3(3) \\
\hline $\mathrm{C}(9)-\mathrm{F}(6)$ & $1.43(3)$ & $\mathrm{C}(16)-\mathrm{P}(1)-\mathrm{C}(14)$ & $111.7(4)$ \\
\hline$C(10)-C(11)$ & $1.509(16)$ & $\mathrm{C}(15)-\mathrm{O}(3)-\mathrm{P}(1)$ & $125.5(8)$ \\
\hline $\mathrm{C}(10)-\mathrm{C}(13)$ & $1.532(12)$ & $\mathrm{C}(1)-\mathrm{N}(1)-\mathrm{S}(1)$ & 113.1(4) \\
\hline $\mathrm{C}(10)-\mathrm{C}(12)$ & $1.540(16)$ & $\mathrm{C}(1)-\mathrm{N}(1)-\mathrm{H}(1 \mathrm{~N})$ & $111(6)$ \\
\hline $\mathrm{C}(11)-\mathrm{H}(11 \mathrm{~A})$ & 0.9700 & $\mathrm{~S}(1)-\mathrm{N}(1)-\mathrm{H}(1 \mathrm{~N})$ & $111(6)$ \\
\hline $\mathrm{C}(11)-\mathrm{H}(11 \mathrm{~B})$ & 0.9700 & $\mathrm{~N}(1)-\mathrm{C}(1)-\mathrm{C}(2)$ & $113.7(5)$ \\
\hline $\mathrm{C}(11)-\mathrm{H}(11 \mathrm{C})$ & 0.9700 & $\mathrm{~N}(1)-\mathrm{C}(1)-\mathrm{C}(14)$ & $107.4(5)$ \\
\hline $\mathrm{C}(12)-\mathrm{H}(12 \mathrm{~A})$ & 0.9700 & $\mathrm{C}(2)-\mathrm{C}(1)-\mathrm{C}(14)$ & $112.9(6)$ \\
\hline $\mathrm{C}(12)-\mathrm{H}(12 \mathrm{~B})$ & 0.9700 & $\mathrm{~N}(1)-\mathrm{C}(1)-\mathrm{H}(1)$ & 107.5 \\
\hline $\mathrm{C}(12)-\mathrm{H}(12 \mathrm{C})$ & 0.9700 & $\mathrm{C}(2)-\mathrm{C}(1)-\mathrm{H}(1)$ & 107.5 \\
\hline $\mathrm{C}(13)-\mathrm{H}(13 \mathrm{~A})$ & 0.9700 & $\mathrm{C}(14)-\mathrm{C}(1)-\mathrm{H}(1)$ & 107.5 \\
\hline $\mathrm{C}(13)-\mathrm{H}(13 \mathrm{~B})$ & 0.9700 & $\mathrm{C}(7)-\mathrm{C}(2)-\mathrm{C}(3)$ & $117.1(7)$ \\
\hline $\mathrm{C}(13)-\mathrm{H}(13 \mathrm{C})$ & 0.9700 & $\mathrm{C}(7)-\mathrm{C}(2)-\mathrm{C}(1)$ & $120.3(7)$ \\
\hline $\mathrm{C}(14)-\mathrm{H}(14 \mathrm{~A})$ & 0.9800 & $\mathrm{C}(3)-\mathrm{C}(2)-\mathrm{C}(1)$ & $122.5(6)$ \\
\hline $\mathrm{C}(14)-\mathrm{H}(14 \mathrm{~B})$ & 0.9800 & $\mathrm{C}(2)-\mathrm{C}(3)-\mathrm{C}(4)$ & $121.7(9)$ \\
\hline$C(15)-H(15 A)$ & 0.9700 & $\mathrm{C}(2)-\mathrm{C}(3)-\mathrm{H}(3)$ & 119.1 \\
\hline $\mathrm{C}(15)-\mathrm{H}(15 \mathrm{~B})$ & 0.9700 & $\mathrm{C}(4)-\mathrm{C}(3)-\mathrm{H}(3)$ & 119.1 \\
\hline$C(15)-H(15 C)$ & 0.9700 & $\mathrm{C}(5)-\mathrm{C}(4)-\mathrm{C}(3)$ & $118.0(11)$ \\
\hline$C(16)-C(17)$ & 1.3900 & $\mathrm{C}(5)-\mathrm{C}(4)-\mathrm{C}(8)$ & $127.4(11)$ \\
\hline$C(16)-C(21)$ & 1.3900 & $\mathrm{C}(3)-\mathrm{C}(4)-\mathrm{C}(8)$ & $114.3(12)$ \\
\hline $\mathrm{C}(17)-\mathrm{C}(18)$ & 1.3900 & $C(6)-C(5)-C(4)$ & 119.4(9) \\
\hline $\mathrm{C}(17)-\mathrm{H}(17)$ & 0.9400 & $\mathrm{C}(6)-\mathrm{C}(5)-\mathrm{H}(5)$ & 120.3 \\
\hline $\mathrm{C}(18)-\mathrm{C}(19)$ & 1.3900 & $\mathrm{C}(4)-\mathrm{C}(5)-\mathrm{H}(5)$ & 120.3 \\
\hline $\mathrm{C}(18)-\mathrm{H}(18)$ & 0.9400 & $C(7)-C(6)-C(5)$ & $121.6(11)$ \\
\hline$C(19)-C(20)$ & 1.3900 & $C(7)-C(6)-C(9)$ & $119.0(15)$ \\
\hline $\mathrm{C}(19)-\mathrm{H}(19)$ & 0.9400 & $\mathrm{C}(5)-\mathrm{C}(6)-\mathrm{C}(9)$ & $119.3(13)$ \\
\hline$C(20)-C(21)$ & 1.3900 & $\mathrm{C}(6)-\mathrm{C}(7)-\mathrm{C}(2)$ & $122.0(10)$ \\
\hline
\end{tabular}




\begin{tabular}{|c|c|c|c|}
\hline $\mathrm{C}(6)-\mathrm{C}(7)-\mathrm{H}(7)$ & 119.0 & $\mathrm{C}(1)-\mathrm{C}(14)-\mathrm{P}(1)$ & $112.8(5)$ \\
\hline $\mathrm{C}(2)-\mathrm{C}(7)-\mathrm{H}(7)$ & 119.0 & $\mathrm{C}(1)-\mathrm{C}(14)-\mathrm{H}(14 \mathrm{~A})$ & 109.0 \\
\hline$F(2)-C(8)-F(3)$ & $122(2)$ & $\mathrm{P}(1)-\mathrm{C}(14)-\mathrm{H}(14 \mathrm{~A})$ & 109.0 \\
\hline $\mathrm{F}(2)-\mathrm{C}(8)-\mathrm{F}(1)$ & $102.8(13)$ & $\mathrm{C}(1)-\mathrm{C}(14)-\mathrm{H}(14 \mathrm{~B})$ & 109.0 \\
\hline $\mathrm{F}(3)-\mathrm{C}(8)-\mathrm{F}(1)$ & $94.9(13)$ & $\mathrm{P}(1)-\mathrm{C}(14)-\mathrm{H}(14 \mathrm{~B})$ & 109.0 \\
\hline $\mathrm{F}(2)-\mathrm{C}(8)-\mathrm{C}(4)$ & $117.0(13)$ & $\mathrm{H}(14 \mathrm{~A})-\mathrm{C}(14)-\mathrm{H}(14 \mathrm{~B})$ & 107.8 \\
\hline $\mathrm{F}(3)-\mathrm{C}(8)-\mathrm{C}(4)$ & $112.8(13)$ & $\mathrm{O}(3)-\mathrm{C}(15)-\mathrm{H}(15 \mathrm{~A})$ & 109.5 \\
\hline $\mathrm{F}(1)-\mathrm{C}(8)-\mathrm{C}(4)$ & $101.2(17)$ & $\mathrm{O}(3)-\mathrm{C}(15)-\mathrm{H}(15 \mathrm{~B})$ & 109.5 \\
\hline $\mathrm{F}(4)-\mathrm{C}(9)-\mathrm{F}(5)$ & $127(2)$ & $H(15 A)-C(15)-H(15 B)$ & 109.5 \\
\hline$F(4)-C(9)-F(6)$ & $99.1(14)$ & $\mathrm{O}(3)-\mathrm{C}(15)-\mathrm{H}(15 \mathrm{C})$ & 109.5 \\
\hline$F(5)-C(9)-F(6)$ & $99.8(14)$ & $\mathrm{H}(15 \mathrm{~A})-\mathrm{C}(15)-\mathrm{H}(15 \mathrm{C})$ & 109.5 \\
\hline $\mathrm{F}(4)-\mathrm{C}(9)-\mathrm{C}(6)$ & $113.0(13)$ & $\mathrm{H}(15 \mathrm{~B})-\mathrm{C}(15)-\mathrm{H}(15 \mathrm{C})$ & 109.5 \\
\hline $\mathrm{F}(5)-\mathrm{C}(9)-\mathrm{C}(6)$ & $109.4(13)$ & $C(17)-C(16)-C(21)$ & 120.0 \\
\hline$F(6)-C(9)-C(6)$ & $104.1(18)$ & $\mathrm{C}(17)-\mathrm{C}(16)-\mathrm{P}(1)$ & $119.2(4)$ \\
\hline$C(11)-C(10)-C(13)$ & $111.1(11)$ & $\mathrm{C}(21)-\mathrm{C}(16)-\mathrm{P}(1)$ & $120.6(4)$ \\
\hline$C(11)-C(10)-C(12)$ & $112.0(10)$ & $\mathrm{C}(18)-\mathrm{C}(17)-\mathrm{C}(16)$ & 120.0 \\
\hline$C(13)-C(10)-C(12)$ & $111.3(10)$ & $\mathrm{C}(18)-\mathrm{C}(17)-\mathrm{H}(17)$ & 120.0 \\
\hline$C(11)-C(10)-S(1)$ & $110.4(7)$ & $\mathrm{C}(16)-\mathrm{C}(17)-\mathrm{H}(17)$ & 120.0 \\
\hline$C(13)-C(10)-S(1)$ & $105.1(7)$ & $\mathrm{C}(17)-\mathrm{C}(18)-\mathrm{C}(19)$ & 120.0 \\
\hline$C(12)-C(10)-S(1)$ & $106.6(7)$ & $\mathrm{C}(17)-\mathrm{C}(18)-\mathrm{H}(18)$ & 120.0 \\
\hline$C(10)-C(11)-H(11 A)$ & 109.5 & $\mathrm{C}(19)-\mathrm{C}(18)-\mathrm{H}(18)$ & 120.0 \\
\hline $\mathrm{C}(10)-\mathrm{C}(11)-\mathrm{H}(11 \mathrm{~B})$ & 109.5 & $C(20)-C(19)-C(18)$ & 120.0 \\
\hline $\mathrm{H}(11 \mathrm{~A})-\mathrm{C}(11)-\mathrm{H}(11 \mathrm{~B})$ & 109.5 & $\mathrm{C}(20)-\mathrm{C}(19)-\mathrm{H}(19)$ & 120.0 \\
\hline $\mathrm{C}(10)-\mathrm{C}(11)-\mathrm{H}(11 \mathrm{C})$ & 109.5 & $\mathrm{C}(18)-\mathrm{C}(19)-\mathrm{H}(19)$ & 120.0 \\
\hline $\mathrm{H}(11 \mathrm{~A})-\mathrm{C}(11)-\mathrm{H}(11 \mathrm{C})$ & 109.5 & $C(19)-C(20)-C(21)$ & 120.0 \\
\hline $\mathrm{H}(11 \mathrm{~B})-\mathrm{C}(11)-\mathrm{H}(11 \mathrm{C})$ & 109.5 & $\mathrm{C}(19)-\mathrm{C}(20)-\mathrm{H}(20)$ & 120.0 \\
\hline $\mathrm{C}(10)-\mathrm{C}(12)-\mathrm{H}(12 \mathrm{~A})$ & 109.5 & $\mathrm{C}(21)-\mathrm{C}(20)-\mathrm{H}(20)$ & 120.0 \\
\hline $\mathrm{C}(10)-\mathrm{C}(12)-\mathrm{H}(12 \mathrm{~B})$ & 109.5 & $C(20)-C(21)-C(16)$ & 120.0 \\
\hline $\mathrm{H}(12 \mathrm{~A})-\mathrm{C}(12)-\mathrm{H}(12 \mathrm{~B})$ & 109.5 & $\mathrm{C}(20)-\mathrm{C}(21)-\mathrm{H}(21)$ & 120.0 \\
\hline $\mathrm{C}(10)-\mathrm{C}(12)-\mathrm{H}(12 \mathrm{C})$ & 109.5 & $C(16)-C(21)-H(21)$ & 120.0 \\
\hline$H(12 A)-C(12)-H(12 C)$ & 109.5 & & \\
\hline $\mathrm{H}(12 \mathrm{~B})-\mathrm{C}(12)-\mathrm{H}(12 \mathrm{C})$ & 109.5 & & \\
\hline $\mathrm{C}(10)-\mathrm{C}(13)-\mathrm{H}(13 \mathrm{~A})$ & 109.5 & & \\
\hline $\mathrm{C}(10)-\mathrm{C}(13)-\mathrm{H}(13 \mathrm{~B})$ & 109.5 & & \\
\hline $\mathrm{H}(13 \mathrm{~A})-\mathrm{C}(13)-\mathrm{H}(13 \mathrm{~B})$ & 109.5 & & \\
\hline $\mathrm{C}(10)-\mathrm{C}(13)-\mathrm{H}(13 \mathrm{C})$ & 109.5 & & \\
\hline $\mathrm{H}(13 \mathrm{~A})-\mathrm{C}(13)-\mathrm{H}(13 \mathrm{C})$ & 109.5 & & \\
\hline $\mathrm{H}(13 \mathrm{~B})-\mathrm{C}(13)-\mathrm{H}(13 \mathrm{C})$ & 109.5 & & \\
\hline
\end{tabular}


Symmetry transformations used to generate equivalent atoms:

Table 4. Anisotropic displacement parameters $\left(\AA^{2} \times 10^{3}\right)$ for compound $9(\boldsymbol{R}, \boldsymbol{S}, \boldsymbol{R})$. The anisotropic displacement factor exponent takes the form: $-2 \pi^{2}\left[\mathrm{~h}^{2} \mathrm{a}^{* 2} \mathrm{U}^{11}+\ldots+2 \mathrm{~h} \mathrm{k} \mathrm{a}^{*}\right.$ $b^{*} \mathrm{U}^{12}$ ]

\begin{tabular}{|c|c|c|c|c|c|c|}
\hline & $\mathrm{U}^{11}$ & $\mathrm{U}^{22}$ & $\mathrm{U}^{33}$ & $\mathrm{U}^{23}$ & $\mathrm{U}^{13}$ & $\mathrm{U}^{12}$ \\
\hline $\mathrm{S}(1)$ & $51(1)$ & $52(1)$ & $33(1)$ & $-3(1)$ & $-10(1)$ & $-5(1)$ \\
\hline $\mathrm{P}(1)$ & $31(1)$ & $126(2)$ & $29(1)$ & $-1(1)$ & $2(1)$ & $17(1)$ \\
\hline $\mathrm{O}(1)$ & $108(5)$ & $56(3)$ & $39(2)$ & $0(2)$ & $5(3)$ & 21(3) \\
\hline $\mathrm{O}(2)$ & $34(2)$ & $115(5)$ & $41(2)$ & $-3(3)$ & $6(2)$ & $8(3)$ \\
\hline $\mathrm{O}(3)$ & $58(3)$ & $140(5)$ & $49(3)$ & $25(3)$ & $8(3)$ & $30(3)$ \\
\hline $\mathrm{N}(1)$ & $34(2)$ & $41(3)$ & $39(2)$ & $-5(2)$ & $-9(2)$ & $-2(2)$ \\
\hline$C(1)$ & $28(3)$ & $63(4)$ & $31(3)$ & $-3(2)$ & $-3(2)$ & $-1(2)$ \\
\hline $\mathrm{C}(2)$ & $44(3)$ & $57(3)$ & $24(2)$ & $0(2)$ & $6(2)$ & $-4(3)$ \\
\hline$C(3)$ & $66(4)$ & $50(4)$ & $40(3)$ & $9(3)$ & $-1(3)$ & $12(3)$ \\
\hline $\mathrm{C}(4)$ & $146(6)$ & $56(5)$ & $53(4)$ & $-1(4)$ & $-10(5)$ & $26(5)$ \\
\hline $\mathrm{C}(5)$ & $195(8)$ & $45(5)$ & $70(6)$ & $-9(4)$ & $17(7)$ & $-8(5)$ \\
\hline$C(6)$ & $142(7)$ & $77(6)$ & $72(6)$ & $-16(5)$ & $40(6)$ & $-52(5)$ \\
\hline$C(7)$ & $61(4)$ & $77(5)$ & $62(4)$ & $-6(4)$ & $23(4)$ & $-26(4)$ \\
\hline $\mathrm{C}(8)$ & 177(9) & 101(8) & 137(9) & $-36(7)$ & $-63(8)$ & $87(7)$ \\
\hline $\mathrm{F}(1)$ & $192(7)$ & $133(5)$ & $205(7)$ & $9(5)$ & $-32(6)$ & $55(5)$ \\
\hline $\mathrm{F}(2)$ & $125(5)$ & 139(6) & $174(6)$ & $-36(5)$ & $-29(5)$ & $55(4)$ \\
\hline $\mathrm{F}(3)$ & $155(6)$ & 181(7) & $160(5)$ & $-42(5)$ & $7(5)$ & $59(6)$ \\
\hline $\mathrm{C}(9)$ & 211(10) & 102(8) & $168(10)$ & $-35(7)$ & $67(9)$ & $-110(8)$ \\
\hline $\mathrm{F}(4)$ & $173(7)$ & $178(7)$ & $178(6)$ & $-32(5)$ & $-23(6)$ & $-52(6)$ \\
\hline $\mathrm{F}(5)$ & $140(6)$ & $148(6)$ & 202(7) & $-16(6)$ & $30(6)$ & $-62(5)$ \\
\hline $\mathrm{F}(6)$ & $185(7)$ & $129(5)$ & $196(7)$ & $2(5)$ & $46(6)$ & $-64(5)$ \\
\hline$C(10)$ & $112(7)$ & $58(4)$ & $46(4)$ & 13(3) & $-13(4)$ & $-4(4)$ \\
\hline $\mathrm{C}(11)$ & 132(8) & $86(8)$ & $88(7)$ & $3(6)$ & $16(7)$ & $-46(7)$ \\
\hline $\mathrm{C}(12)$ & $147(9)$ & $49(5)$ & $91(7)$ & $12(5)$ & $-9(7)$ & $24(6)$ \\
\hline $\mathrm{C}(13)$ & 211(14) & $60(6)$ & $45(4)$ & $9(3)$ & $-26(6)$ & $6(7)$ \\
\hline$C(14)$ & $35(3)$ & $76(5)$ & $34(3)$ & $-18(3)$ & $-1(2)$ & $-6(3)$ \\
\hline$C(15)$ & $134(12)$ & 127(9) & 141(13) & 11(9) & $43(11)$ & $5(10)$ \\
\hline$C(16)$ & $49(4)$ & $136(6)$ & $49(3)$ & $-4(4)$ & $5(3)$ & $2(4)$ \\
\hline
\end{tabular}




\begin{tabular}{lcccccc}
$\mathrm{C}(17)$ & $76(5)$ & $146(7)$ & $72(5)$ & $-27(5)$ & $20(4)$ & $8(5)$ \\
$\mathrm{C}(18)$ & $116(6)$ & $166(8)$ & $100(6)$ & $-37(6)$ & $-8(6)$ & $8(6)$ \\
$\mathrm{C}(19)$ & $117(7)$ & $184(9)$ & $99(6)$ & $-40(6)$ & $-5(5)$ & $2(7)$ \\
$\mathrm{C}(20)$ & $111(6)$ & $170(8)$ & $104(6)$ & $-31(6)$ & $-13(5)$ & $7(6)$ \\
$\mathrm{C}(21)$ & $58(4)$ & $140(7)$ & $64(4)$ & $-16(5)$ & $-4(3)$ & $5(5)$ \\
\hline
\end{tabular}

Table 5. Hydrogen coordinates $\left(\times 10^{4}\right)$ and isotropic displacement parameters $\left(\AA^{2} \times 10^{3}\right)$ for compound $\mathbf{9}(\boldsymbol{R}, \boldsymbol{S}, \boldsymbol{R})$.

\begin{tabular}{|c|c|c|c|c|}
\hline & $\mathrm{x}$ & $\mathrm{y}$ & $\mathrm{z}$ & $\mathrm{U}(\mathrm{eq})$ \\
\hline $\mathrm{H}(1 \mathrm{~N})$ & $5980(20)$ & $3190(80)$ & $9080(40)$ & 56 \\
\hline $\mathrm{H}(1)$ & 3602 & 2641 & 9498 & 49 \\
\hline $\mathrm{H}(3)$ & 6620 & 942 & 9386 & 62 \\
\hline $\mathrm{H}(5)$ & 4846 & -2376 & 9109 & 124 \\
\hline $\mathrm{H}(7)$ & 2754 & 703 & 9379 & 80 \\
\hline $\mathrm{H}(11 \mathrm{~A})$ & 6899 & 4268 & 8039 & 153 \\
\hline $\mathrm{H}(11 \mathrm{~B})$ & 6355 & 5102 & 8556 & 153 \\
\hline $\mathrm{H}(11 \mathrm{C})$ & 6410 & 5647 & 7913 & 153 \\
\hline $\mathrm{H}(12 \mathrm{~A})$ & 3968 & 5341 & 8702 & 143 \\
\hline $\mathrm{H}(12 \mathrm{~B})$ & 3010 & 4935 & 8195 & 143 \\
\hline $\mathrm{H}(12 \mathrm{C})$ & 3941 & 6100 & 8108 & 143 \\
\hline $\mathrm{H}(13 \mathrm{~A})$ & 5510 & 4296 & 7169 & 158 \\
\hline $\mathrm{H}(13 \mathrm{~B})$ & 4051 & 4746 & 7230 & 158 \\
\hline $\mathrm{H}(13 \mathrm{C})$ & 4405 & 3317 & 7320 & 158 \\
\hline $\mathrm{H}(14 \mathrm{~A})$ & 4972 & 3712 & 10153 & 58 \\
\hline $\mathrm{H}(14 \mathrm{~B})$ & 6023 & 2635 & 10105 & 58 \\
\hline $\mathrm{H}(15 \mathrm{~A})$ & 5546 & -864 & 11085 & 200 \\
\hline $\mathrm{H}(15 \mathrm{~B})$ & 4162 & -906 & 10784 & 200 \\
\hline $\mathrm{H}(15 \mathrm{C})$ & 4365 & -131 & 11367 & 200 \\
\hline $\mathrm{H}(17)$ & 2957 & 3207 & 11584 & 117 \\
\hline $\mathrm{H}(18)$ & 3553 & 4221 & 12444 & 153 \\
\hline $\mathrm{H}(19)$ & 5719 & 4240 & 12738 & 160 \\
\hline $\mathrm{H}(20)$ & 7289 & 3245 & 12171 & 154 \\
\hline $\mathrm{H}(21)$ & 6693 & 2231 & 11310 & 105 \\
\hline
\end{tabular}


Table 6. Torsion angles $\left[{ }^{\circ}\right]$ for compound $\mathbf{9}(\boldsymbol{R}, \boldsymbol{S}, \boldsymbol{R})$.

\begin{tabular}{|c|c|}
\hline $\mathrm{O}(2)-\mathrm{P}(1)-\mathrm{O}(3)-\mathrm{C}(15)$ & $-50.6(13)$ \\
\hline $\mathrm{C}(16)-\mathrm{P}(1)-\mathrm{O}(3)-\mathrm{C}(15)$ & $72.3(13)$ \\
\hline $\mathrm{C}(14)-\mathrm{P}(1)-\mathrm{O}(3)-\mathrm{C}(15)$ & $-171.9(12)$ \\
\hline $\mathrm{O}(1)-\mathrm{S}(1)-\mathrm{N}(1)-\mathrm{C}(1)$ & $-96.7(5)$ \\
\hline $\mathrm{C}(10)-\mathrm{S}(1)-\mathrm{N}(1)-\mathrm{C}(1)$ & $151.9(5)$ \\
\hline$S(1)-N(1)-C(1)-C(2)$ & $52.9(6)$ \\
\hline $\mathrm{S}(1)-\mathrm{N}(1)-\mathrm{C}(1)-\mathrm{C}(14)$ & $178.5(4)$ \\
\hline $\mathrm{N}(1)-\mathrm{C}(1)-\mathrm{C}(2)-\mathrm{C}(7)$ & $-123.0(7)$ \\
\hline$C(14)-C(1)-C(2)-C(7)$ & $114.3(7)$ \\
\hline $\mathrm{N}(1)-\mathrm{C}(1)-\mathrm{C}(2)-\mathrm{C}(3)$ & $60.3(8)$ \\
\hline $\mathrm{C}(14)-\mathrm{C}(1)-\mathrm{C}(2)-\mathrm{C}(3)$ & $-62.4(8)$ \\
\hline $\mathrm{C}(7)-\mathrm{C}(2)-\mathrm{C}(3)-\mathrm{C}(4)$ & $2.3(10)$ \\
\hline$C(1)-C(2)-C(3)-C(4)$ & $179.1(7)$ \\
\hline$C(2)-C(3)-C(4)-C(5)$ & $-0.8(12)$ \\
\hline$C(2)-C(3)-C(4)-C(8)$ & $174.3(9)$ \\
\hline$C(3)-C(4)-C(5)-C(6)$ & $1.4(14)$ \\
\hline$C(8)-C(4)-C(5)-C(6)$ & $-173.0(13)$ \\
\hline$C(4)-C(5)-C(6)-C(7)$ & $-3.8(16)$ \\
\hline$C(4)-C(5)-C(6)-C(9)$ & $174.0(12)$ \\
\hline$C(5)-C(6)-C(7)-C(2)$ & $5.5(16)$ \\
\hline$C(9)-C(6)-C(7)-C(2)$ & $-172.3(11)$ \\
\hline$C(3)-C(2)-C(7)-C(6)$ & $-4.6(12)$ \\
\hline$C(1)-C(2)-C(7)-C(6)$ & $178.5(8)$ \\
\hline $\mathrm{C}(5)-\mathrm{C}(4)-\mathrm{C}(8)-\mathrm{F}(2)$ & $-146.6(16)$ \\
\hline $\mathrm{C}(3)-\mathrm{C}(4)-\mathrm{C}(8)-\mathrm{F}(2)$ & $39(2)$ \\
\hline $\mathrm{C}(5)-\mathrm{C}(4)-\mathrm{C}(8)-\mathrm{F}(3)$ & $64(2)$ \\
\hline$C(3)-C(4)-C(8)-F(3)$ & $-110.2(16)$ \\
\hline $\mathrm{C}(5)-\mathrm{C}(4)-\mathrm{C}(8)-\mathrm{F}(1)$ & $-35.9(17)$ \\
\hline $\mathrm{C}(3)-\mathrm{C}(4)-\mathrm{C}(8)-\mathrm{F}(1)$ & $149.5(10)$ \\
\hline$C(7)-C(6)-C(9)-F(4)$ & $106.2(18)$ \\
\hline $\mathrm{C}(5)-\mathrm{C}(6)-\mathrm{C}(9)-\mathrm{F}(4)$ & $-72(2)$ \\
\hline $\mathrm{C}(7)-\mathrm{C}(6)-\mathrm{C}(9)-\mathrm{F}(5)$ & $-41(2)$ \\
\hline $\mathrm{C}(5)-\mathrm{C}(6)-\mathrm{C}(9)-\mathrm{F}(5)$ & $140.7(16)$ \\
\hline$C(7)-C(6)-C(9)-F(6)$ & $-147.4(12)$ \\
\hline $\mathrm{C}(5)-\mathrm{C}(6)-\mathrm{C}(9)-\mathrm{F}(6)$ & $34.8(17)$ \\
\hline $\mathrm{O}(1)-\mathrm{S}(1)-\mathrm{C}(10)-\mathrm{C}(11)$ & $-62.2(8)$ \\
\hline
\end{tabular}




$\begin{array}{lc}\mathrm{N}(1)-\mathrm{S}(1)-\mathrm{C}(10)-\mathrm{C}(11) & 51.9(8) \\ \mathrm{O}(1)-\mathrm{S}(1)-\mathrm{C}(10)-\mathrm{C}(13) & 57.8(10) \\ \mathrm{N}(1)-\mathrm{S}(1)-\mathrm{C}(10)-\mathrm{C}(13) & 171.8(9) \\ \mathrm{O}(1)-\mathrm{S}(1)-\mathrm{C}(10)-\mathrm{C}(12) & 176.0(7) \\ \mathrm{N}(1)-\mathrm{S}(1)-\mathrm{C}(10)-\mathrm{C}(12) & -70.0(7) \\ \mathrm{N}(1)-\mathrm{C}(1)-\mathrm{C}(14)-\mathrm{P}(1) & 175.7(4) \\ \mathrm{C}(2)-\mathrm{C}(1)-\mathrm{C}(14)-\mathrm{P}(1) & -58.2(7) \\ \mathrm{O}(2)-\mathrm{P}(1)-\mathrm{C}(14)-\mathrm{C}(1) & -42.5(7) \\ \mathrm{O}(3)-\mathrm{P}(1)-\mathrm{C}(14)-\mathrm{C}(1) & 80.6(6) \\ \mathrm{C}(16)-\mathrm{P}(1)-\mathrm{C}(14)-\mathrm{C}(1) & -169.9(5) \\ \mathrm{O}(2)-\mathrm{P}(1)-\mathrm{C}(16)-\mathrm{C}(17) & -13.8(7) \\ \mathrm{O}(3)-\mathrm{P}(1)-\mathrm{C}(16)-\mathrm{C}(17) & -138.4(5) \\ \mathrm{C}(14)-\mathrm{P}(1)-\mathrm{C}(16)-\mathrm{C}(17) & 113.5(6) \\ \mathrm{O}(2)-\mathrm{P}(1)-\mathrm{C}(16)-\mathrm{C}(21) & 172.1(6) \\ \mathrm{O}(3)-\mathrm{P}(1)-\mathrm{C}(16)-\mathrm{C}(21) & 47.6(6) \\ \mathrm{C}(14)-\mathrm{P}(1)-\mathrm{C}(16)-\mathrm{C}(21) & -60.5(7) \\ \mathrm{C}(21)-\mathrm{C}(16)-\mathrm{C}(17)-\mathrm{C}(18) & 0.0 \\ \mathrm{P}(1)-\mathrm{C}(16)-\mathrm{C}(17)-\mathrm{C}(18) & -174.1(7) \\ \mathrm{C}(16)-\mathrm{C}(17)-\mathrm{C}(18)-\mathrm{C}(19) & 0.0 \\ \mathrm{C}(17)-\mathrm{C}(18)-\mathrm{C}(19)-\mathrm{C}(20) & 0.0 \\ \mathrm{C}(18)-\mathrm{C}(19)-\mathrm{C}(20)-\mathrm{C}(21) & 0.0 \\ \mathrm{C}(19)-\mathrm{C}(20)-\mathrm{C}(21)-\mathrm{C}(16) & 0.0 \\ \mathrm{C}(17)-\mathrm{C}(16)-\mathrm{C}(21)-\mathrm{C}(20) & 0.0 \\ \mathrm{P}(1)-\mathrm{C}(16)-\mathrm{C}(21)-\mathrm{C}(20) & \\ & 0(7) \\ & \\ & \\ & \end{array}$

Symmetry transformations used to generate equivalent atoms:

Table 7. Hydrogen bonds for compound $\mathbf{9}(\boldsymbol{R}, S, S)\left[\AA\right.$ and $\left.^{\circ}\right]$.

\begin{tabular}{lllll}
\hline D-H...A & d(D-H) & d(H...A & $d(D . . A)$ & $<($ DHA $)$ \\
\hline $\mathrm{N}(1)-\mathrm{H}(1 \mathrm{~N}) \ldots \mathrm{O}(2) \# 1$ & $0.84(2)$ & $2.10(3)$ & $2.913(7)$ & $165(9)$ \\
\hline
\end{tabular}

Symmetry transformations used to generate equivalent atoms:

$\# 1 \mathrm{x}+1 / 2,-\mathrm{y}+1 / 2,-\mathrm{z}+2$ 

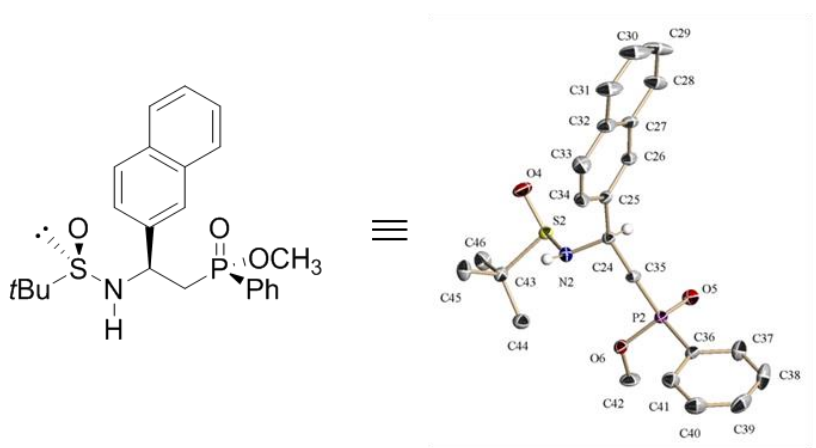

Table 1. Crystal data and structure refinement for compound $10(R, S, S)$.

Empirical formula

Formula weight

Temperature

Wavelength

Crystal system

Space group

Unit cell dimensions

Volume

$\mathrm{Z}$

Density (calculated)

Absorption coefficient

$\mathrm{F}(000)$

Crystal size

Theta range for data collection

Index ranges

Reflections collected

Independent reflections

Completeness to theta $=25.24^{\circ}$

Absorption correction

Max. and min. transmission

Refinement method

Data / restraints / parameters

Goodness-of-fit on $\mathrm{F}^{2}$

Final $\mathrm{R}$ indices [I $>2 \operatorname{sigma}(\mathrm{I})]$

$\mathrm{R}$ indices (all data)
$\mathrm{C}_{23} \mathrm{H}_{28} \mathrm{NO}_{3} \mathrm{PS}$

429.49

213(2) K

$0.71073 \AA$

Monoclinic

$\mathrm{C}_{2}$

$\mathrm{a}=36.035(2) \AA$

$\alpha=90^{\circ}$

$\mathrm{b}=5.9026(4) \AA$ $\beta=114.708(3)^{\circ}$.

$\mathrm{c}=24.7204(14) \AA$ $\gamma=90^{\circ}$.

4776.7(5) $\AA^{3}$

8

$1.194 \mathrm{Mg} / \mathrm{m}^{3}$

$0.225 \mathrm{~mm}^{-1}$

1824

$0.30 \times 0.20 \times 0.10 \mathrm{~mm}^{3}$

2.49 to $25.24^{\circ}$.

$-42<=\mathrm{h}<=41,-7<=\mathrm{k}<=5,-29<=\mathrm{l}<=29$

25097

$6721[\mathrm{R}(\mathrm{int})=0.0455]$

$99.5 \%$

Semi-empirical from equivalents

0.9779 and 0.9467

Full-matrix-block least-squares on $\mathrm{F}^{2}$

$6721 / 3 / 537$

1.087

$\mathrm{R} 1=0.0546, \mathrm{wR} 2=0.1383$

$\mathrm{R} 1=0.0618, \mathrm{wR} 2=0.1416$ 
Table 2. Atomic coordinates $\left(\times 10^{4}\right)$ and equivalent isotropic displacement parameters $\left(\AA^{2} \times 10^{3}\right)$ for compound $\mathbf{1 0}(\boldsymbol{R}, S, S)$. U(eq) is defined as one third of the trace of the orthogonalized $\mathrm{U}^{\mathrm{ij}}$ tensor.

\begin{tabular}{|c|c|c|c|c|}
\hline & $\mathrm{x}$ & $\mathrm{y}$ & $\mathrm{z}$ & $\mathrm{U}(\mathrm{eq})$ \\
\hline$S(1)$ & $8646(1)$ & $7516(3)$ & $8808(1)$ & $59(1)$ \\
\hline $\mathrm{P}(1)$ & $7469(1)$ & $11810(2)$ & $8774(1)$ & $29(1)$ \\
\hline $\mathrm{O}(1)$ & $8662(2)$ & $5524(13)$ & $8457(2)$ & $124(3)$ \\
\hline $\mathrm{O}(2)$ & $7624(1)$ & 14071(6) & $8728(2)$ & $47(1)$ \\
\hline $\mathrm{O}(3)$ & $7702(1)$ & $10550(6)$ & 9391(1) & $39(1)$ \\
\hline $\mathrm{N}(1)$ & $8170(1)$ & $7964(7)$ & $8741(2)$ & $31(1)$ \\
\hline $\mathrm{C}(1)$ & $7928(1)$ & $9467(9)$ & $8245(2)$ & $31(1)$ \\
\hline $\mathrm{C}(2)$ & $7860(1)$ & $8612(8)$ & $7632(2)$ & $31(1)$ \\
\hline $\mathrm{C}(3)$ & 7967(1) & $9927(8)$ & $7269(2)$ & $31(1)$ \\
\hline$C(4)$ & 7899(1) & 9211(8) & $6683(2)$ & $30(1)$ \\
\hline$C(5)$ & 8013(1) & 10484(9) & $6299(2)$ & $42(1)$ \\
\hline$C(6)$ & $7956(2)$ & $9735(10)$ & $5756(2)$ & $45(1)$ \\
\hline$C(7)$ & 7761(1) & $7616(10)$ & $5547(2)$ & 41(1) \\
\hline $\mathrm{C}(8)$ & $7642(1)$ & $6326(9)$ & $5900(2)$ & $37(1)$ \\
\hline $\mathrm{C}(9)$ & $7712(1)$ & $7077(8)$ & $6482(2)$ & $32(1)$ \\
\hline $\mathrm{C}(10)$ & $7612(1)$ & $5728(9)$ & $6877(2)$ & $33(1)$ \\
\hline $\mathrm{C}(11)$ & $7687(1)$ & $6443(8)$ & $7429(2)$ & $33(1)$ \\
\hline $\mathrm{C}(12)$ & $7500(1)$ & $9762(9)$ & $8257(2)$ & $34(1)$ \\
\hline$C(13)$ & 6941(1) & $11920(8)$ & $8648(2)$ & $26(1)$ \\
\hline$C(14)$ & $6712(2)$ & 13857(9) & $8406(2)$ & $39(1)$ \\
\hline$C(15)$ & $6311(2)$ & $13965(11)$ & $8319(2)$ & $52(1)$ \\
\hline$C(16)$ & $6130(2)$ & $12154(11)$ & $8462(2)$ & $50(2)$ \\
\hline $\mathrm{C}(17)$ & $6350(2)$ & 10193(10) & $8691(2)$ & $44(1)$ \\
\hline $\mathrm{C}(18)$ & $6756(1)$ & 10071(9) & $8788(2)$ & $35(1)$ \\
\hline$C(19)$ & $7794(2)$ & $11673(12)$ & $9939(2)$ & $58(2)$ \\
\hline $\mathrm{C}(20)$ & 8874(1) & $6555(9)$ & $9580(2)$ & $40(1)$ \\
\hline $\mathrm{C}(21)$ & $8870(2)$ & $8508(16)$ & $9958(3)$ & $94(3)$ \\
\hline$C(22)$ & $8650(2)$ & $4543(15)$ & $9668(3)$ & $96(3)$ \\
\hline
\end{tabular}




\begin{tabular}{|c|c|c|c|c|}
\hline$C(23)$ & $9311(2)$ & $5983(17)$ & $9720(3)$ & $94(3)$ \\
\hline$S(2)$ & $5138(1)$ & $7451(2)$ & 8293(1) & $30(1)$ \\
\hline $\mathrm{P}(2)$ & $4272(1)$ & $10812(2)$ & 6584(1) & $26(1)$ \\
\hline $\mathrm{O}(4)$ & 5533(1) & $6263(8)$ & $8560(2)$ & $54(1)$ \\
\hline $\mathrm{O}(5)$ & 4463(1) & 13010(6) & $6819(1)$ & $39(1)$ \\
\hline $\mathrm{O}(6)$ & $4040(1)$ & $9650(6)$ & 6932(1) & $34(1)$ \\
\hline $\mathrm{N}(2)$ & 4890(1) & 6992(7) & $7572(1)$ & $30(1)$ \\
\hline $\mathrm{C}(24)$ & $5004(1)$ & $8317(8)$ & $7168(2)$ & $27(1)$ \\
\hline$C(25)$ & $5344(1)$ & $7319(8)$ & 7033(2) & $29(1)$ \\
\hline$C(26)$ & 5691(1) & $8590(9)$ & $7144(2)$ & $32(1)$ \\
\hline$C(27)$ & 5992(1) & $7764(10)$ & $6969(2)$ & $38(1)$ \\
\hline$C(28)$ & $6344(1)$ & $9093(12)$ & $7052(2)$ & $53(2)$ \\
\hline$C(29)$ & $6621(2)$ & $8286(15)$ & $6843(2)$ & $75(2)$ \\
\hline$C(30)$ & $6565(2)$ & $6227(16)$ & $6568(2)$ & $79(3)$ \\
\hline$C(31)$ & $6241(2)$ & $4871(13)$ & $6488(2)$ & $62(2)$ \\
\hline$C(32)$ & 5939(1) & $5658(11)$ & 6691(2) & $43(1)$ \\
\hline$C(33)$ & $5589(2)$ & $4352(10)$ & $6602(2)$ & $48(1)$ \\
\hline $\mathrm{C}(34)$ & 5301(1) & $5182(8)$ & $6768(2)$ & $36(1)$ \\
\hline$C(35)$ & $4621(1)$ & $8676(8)$ & $6578(2)$ & $27(1)$ \\
\hline$C(36)$ & 3902(1) & 11081(8) & $5824(2)$ & $30(1)$ \\
\hline$C(37)$ & $3895(2)$ & 13039(10) & $5506(2)$ & $47(1)$ \\
\hline $\mathrm{C}(38)$ & $3622(2)$ & $13204(11)$ & 4903(2) & $63(2)$ \\
\hline $\mathrm{C}(39)$ & $3363(2)$ & $11436(12)$ & $4628(2)$ & $60(2)$ \\
\hline$C(40)$ & $3366(2)$ & $9537(12)$ & 4934(2) & $56(2)$ \\
\hline $\mathrm{C}(41)$ & $3638(1)$ & $9344(10)$ & $5539(2)$ & $43(1)$ \\
\hline$C(42)$ & $3769(1)$ & $10998(11)$ & $7089(2)$ & $50(1)$ \\
\hline $\mathrm{C}(43)$ & $4795(1)$ & 5872(9) & $8541(2)$ & $31(1)$ \\
\hline$C(44)$ & $4362(1)$ & $6775(11)$ & $8220(2)$ & $45(1)$ \\
\hline $\mathrm{C}(45)$ & 4819(2) & $3341(9)$ & $8443(2)$ & $47(1)$ \\
\hline$C(46)$ & 4967(2) & $6415(10)$ & $9214(2)$ & $47(1)$ \\
\hline
\end{tabular}


Table 3. Bond lengths $[\AA]$ and angles $\left[{ }^{\circ}\right]$ for compound $\mathbf{1 0}(\boldsymbol{R}, S, S)$.

\begin{tabular}{|c|c|}
\hline $\mathrm{S}(1)-\mathrm{O}(1)$ & $1.478(6)$ \\
\hline $\mathrm{S}(1)-\mathrm{N}(1)$ & $1.671(4)$ \\
\hline$S(1)-C(20)$ & $1.824(5)$ \\
\hline $\mathrm{P}(1)-\mathrm{O}(2)$ & $1.469(4)$ \\
\hline $\mathrm{P}(1)-\mathrm{O}(3)$ & $1.587(3)$ \\
\hline $\mathrm{P}(1)-\mathrm{C}(12)$ & $1.795(4)$ \\
\hline$P(1)-C(13)$ & $1.797(4)$ \\
\hline $\mathrm{O}(3)-\mathrm{C}(19)$ & $1.418(6)$ \\
\hline $\mathrm{N}(1)-\mathrm{C}(1)$ & $1.468(6)$ \\
\hline $\mathrm{N}(1)-\mathrm{H}(1 \mathrm{~N})$ & $0.89(2)$ \\
\hline$C(1)-C(2)$ & $1.517(6)$ \\
\hline $\mathrm{C}(1)-\mathrm{C}(12)$ & $1.564(6)$ \\
\hline $\mathrm{C}(1)-\mathrm{H}(1)$ & 0.9900 \\
\hline$C(2)-C(3)$ & $1.359(6)$ \\
\hline $\mathrm{C}(2)-\mathrm{C}(11)$ & $1.421(7)$ \\
\hline$C(3)-C(4)$ & $1.428(6)$ \\
\hline $\mathrm{C}(3)-\mathrm{H}(3)$ & 0.9400 \\
\hline$C(4)-C(5)$ & $1.400(6)$ \\
\hline$C(4)-C(9)$ & $1.416(7)$ \\
\hline$C(5)-C(6)$ & $1.344(7)$ \\
\hline $\mathrm{C}(5)-\mathrm{H}(5)$ & 0.9400 \\
\hline$C(6)-C(7)$ & $1.421(8)$ \\
\hline $\mathrm{C}(6)-\mathrm{H}(6)$ & 0.9400 \\
\hline$C(7)-C(8)$ & $1.356(7)$ \\
\hline $\mathrm{C}(7)-\mathrm{H}(7)$ & 0.9400 \\
\hline $\mathrm{C}(8)-\mathrm{C}(9)$ & $1.422(6)$ \\
\hline $\mathrm{C}(8)-\mathrm{H}(8)$ & 0.9400 \\
\hline $\mathrm{C}(9)-\mathrm{C}(10)$ & $1.419(6)$ \\
\hline$C(10)-C(11)$ & $1.343(6)$ \\
\hline $\mathrm{C}(10)-\mathrm{H}(10)$ & 0.9400 \\
\hline $\mathrm{C}(11)-\mathrm{H}(11)$ & 0.9400 \\
\hline $\mathrm{C}(12)-\mathrm{H}(12 \mathrm{~A})$ & 0.9800 \\
\hline $\mathrm{C}(12)-\mathrm{H}(12 \mathrm{~B})$ & 0.9800 \\
\hline $\mathrm{C}(13)-\mathrm{C}(14)$ & $1.391(7)$ \\
\hline $\mathrm{C}(13)-\mathrm{C}(18)$ & $1.397(6)$ \\
\hline $\mathrm{C}(14)-\mathrm{C}(15)$ & $1.370(7)$ \\
\hline
\end{tabular}




\begin{tabular}{|c|c|}
\hline $\mathrm{C}(14)-\mathrm{H}(14)$ & 0.9400 \\
\hline$C(15)-C(16)$ & $1.375(9)$ \\
\hline $\mathrm{C}(15)-\mathrm{H}(15)$ & 0.9400 \\
\hline$C(16)-C(17)$ & $1.384(8)$ \\
\hline $\mathrm{C}(16)-\mathrm{H}(16)$ & 0.9400 \\
\hline $\mathrm{C}(17)-\mathrm{C}(18)$ & $1.380(6)$ \\
\hline $\mathrm{C}(17)-\mathrm{H}(17)$ & 0.9400 \\
\hline $\mathrm{C}(18)-\mathrm{H}(18)$ & 0.9400 \\
\hline $\mathrm{C}(19)-\mathrm{H}(19 \mathrm{~A})$ & 0.9700 \\
\hline $\mathrm{C}(19)-\mathrm{H}(19 \mathrm{~B})$ & 0.9700 \\
\hline $\mathrm{C}(19)-\mathrm{H}(19 \mathrm{C})$ & 0.9700 \\
\hline$C(20)-C(21)$ & $1.489(9)$ \\
\hline$C(20)-C(23)$ & $1.502(7)$ \\
\hline$C(20)-C(22)$ & $1.503(9)$ \\
\hline $\mathrm{C}(21)-\mathrm{H}(21 \mathrm{~A})$ & 0.9700 \\
\hline $\mathrm{C}(21)-\mathrm{H}(21 \mathrm{~B})$ & 0.9700 \\
\hline $\mathrm{C}(21)-\mathrm{H}(21 \mathrm{C})$ & 0.9700 \\
\hline $\mathrm{C}(22)-\mathrm{H}(22 \mathrm{~A})$ & 0.9700 \\
\hline $\mathrm{C}(22)-\mathrm{H}(22 \mathrm{~B})$ & 0.9700 \\
\hline $\mathrm{C}(22)-\mathrm{H}(22 \mathrm{C})$ & 0.9700 \\
\hline $\mathrm{C}(23)-\mathrm{H}(23 \mathrm{~A})$ & 0.9700 \\
\hline $\mathrm{C}(23)-\mathrm{H}(23 \mathrm{~B})$ & 0.9700 \\
\hline $\mathrm{C}(23)-\mathrm{H}(23 \mathrm{C})$ & 0.9700 \\
\hline $\mathrm{S}(2)-\mathrm{O}(4)$ & $1.473(3)$ \\
\hline $\mathrm{S}(2)-\mathrm{N}(2)$ & $1.648(3)$ \\
\hline$S(2)-C(43)$ & $1.845(5)$ \\
\hline $\mathrm{P}(2)-\mathrm{O}(5)$ & $1.470(4)$ \\
\hline $\mathrm{P}(2)-\mathrm{O}(6)$ & $1.585(3)$ \\
\hline $\mathrm{P}(2)-\mathrm{C}(35)$ & $1.785(4)$ \\
\hline $\mathrm{P}(2)-\mathrm{C}(36)$ & $1.799(4)$ \\
\hline $\mathrm{O}(6)-\mathrm{C}(42)$ & $1.435(6)$ \\
\hline $\mathrm{N}(2)-\mathrm{C}(24)$ & $1.456(5)$ \\
\hline $\mathrm{N}(2)-\mathrm{H}(2 \mathrm{~N})$ & $0.90(2)$ \\
\hline$C(24)-C(25)$ & $1.517(5)$ \\
\hline$C(24)-C(35)$ & $1.546(5)$ \\
\hline $\mathrm{C}(24)-\mathrm{H}(24)$ & 0.9900 \\
\hline$C(25)-C(26)$ & $1.384(6)$ \\
\hline $\mathrm{C}(25)-\mathrm{C}(34)$ & $1.399(7)$ \\
\hline
\end{tabular}




\begin{tabular}{|c|c|}
\hline$C(26)-C(27)$ & $1.414(6)$ \\
\hline $\mathrm{C}(26)-\mathrm{H}(26)$ & 0.9400 \\
\hline $\mathrm{C}(27)-\mathrm{C}(32)$ & $1.394(8)$ \\
\hline $\mathrm{C}(27)-\mathrm{C}(28)$ & $1.430(7)$ \\
\hline $\mathrm{C}(28)-\mathrm{C}(29)$ & $1.388(7)$ \\
\hline $\mathrm{C}(28)-\mathrm{H}(28)$ & 0.9400 \\
\hline $\mathrm{C}(29)-\mathrm{C}(30)$ & $1.366(11)$ \\
\hline $\mathrm{C}(29)-\mathrm{H}(29)$ & 0.9400 \\
\hline $\mathrm{C}(30)-\mathrm{C}(31)$ & $1.361(10)$ \\
\hline $\mathrm{C}(30)-\mathrm{H}(30)$ & 0.9400 \\
\hline $\mathrm{C}(31)-\mathrm{C}(32)$ & $1.449(6)$ \\
\hline $\mathrm{C}(31)-\mathrm{H}(31)$ & 0.9400 \\
\hline $\mathrm{C}(32)-\mathrm{C}(33)$ & $1.416(8)$ \\
\hline $\mathrm{C}(33)-\mathrm{C}(34)$ & $1.359(6)$ \\
\hline $\mathrm{C}(33)-\mathrm{H}(33)$ & 0.9400 \\
\hline $\mathrm{C}(34)-\mathrm{H}(34)$ & 0.9400 \\
\hline $\mathrm{C}(35)-\mathrm{H}(35 \mathrm{~A})$ & 0.9800 \\
\hline $\mathrm{C}(35)-\mathrm{H}(35 \mathrm{~B})$ & 0.9800 \\
\hline$C(36)-C(41)$ & $1.376(7)$ \\
\hline$C(36)-C(37)$ & $1.392(7)$ \\
\hline $\mathrm{C}(37)-\mathrm{C}(38)$ & $1.405(7)$ \\
\hline $\mathrm{C}(37)-\mathrm{H}(37)$ & 0.9400 \\
\hline $\mathrm{C}(38)-\mathrm{C}(39)$ & $1.375(9)$ \\
\hline $\mathrm{C}(38)-\mathrm{H}(38)$ & 0.9400 \\
\hline$C(39)-C(40)$ & $1.349(9)$ \\
\hline C(39)-H(39) & 0.9400 \\
\hline$C(40)-C(41)$ & $1.409(7)$ \\
\hline $\mathrm{C}(40)-\mathrm{H}(40)$ & 0.9400 \\
\hline $\mathrm{C}(41)-\mathrm{H}(41)$ & 0.9400 \\
\hline $\mathrm{C}(42)-\mathrm{H}(42 \mathrm{~A})$ & 0.9700 \\
\hline $\mathrm{C}(42)-\mathrm{H}(42 \mathrm{~B})$ & 0.9700 \\
\hline $\mathrm{C}(42)-\mathrm{H}(42 \mathrm{C})$ & 0.9700 \\
\hline$C(43)-C(45)$ & $1.521(7)$ \\
\hline$C(43)-C(44)$ & $1.522(6)$ \\
\hline$C(43)-C(46)$ & $1.546(6)$ \\
\hline $\mathrm{C}(44)-\mathrm{H}(44 \mathrm{~A})$ & 0.9700 \\
\hline $\mathrm{C}(44)-\mathrm{H}(44 \mathrm{~B})$ & 0.9700 \\
\hline $\mathrm{C}(44)-\mathrm{H}(44 \mathrm{C})$ & 0.9700 \\
\hline
\end{tabular}




\begin{tabular}{|c|c|}
\hline $\mathrm{C}(45)-\mathrm{H}(45 \mathrm{~A})$ & 0.9700 \\
\hline $\mathrm{C}(45)-\mathrm{H}(45 \mathrm{~B})$ & 0.9700 \\
\hline $\mathrm{C}(45)-\mathrm{H}(45 \mathrm{C})$ & 0.9700 \\
\hline $\mathrm{C}(46)-\mathrm{H}(46 \mathrm{~A})$ & 0.9700 \\
\hline $\mathrm{C}(46)-\mathrm{H}(46 \mathrm{~B})$ & 0.9700 \\
\hline $\mathrm{C}(46)-\mathrm{H}(46 \mathrm{C})$ & 0.9700 \\
\hline $\mathrm{O}(1)-\mathrm{S}(1)-\mathrm{N}(1)$ & $111.0(3)$ \\
\hline $\mathrm{O}(1)-\mathrm{S}(1)-\mathrm{C}(20)$ & $104.8(3)$ \\
\hline $\mathrm{N}(1)-\mathrm{S}(1)-\mathrm{C}(20)$ & $98.67(19)$ \\
\hline $\mathrm{O}(2)-\mathrm{P}(1)-\mathrm{O}(3)$ & $115.8(2)$ \\
\hline $\mathrm{O}(2)-\mathrm{P}(1)-\mathrm{C}(12)$ & $114.8(2)$ \\
\hline $\mathrm{O}(3)-\mathrm{P}(1)-\mathrm{C}(12)$ & 101.7(2) \\
\hline $\mathrm{O}(2)-\mathrm{P}(1)-\mathrm{C}(13)$ & $111.3(2)$ \\
\hline $\mathrm{O}(3)-\mathrm{P}(1)-\mathrm{C}(13)$ & $106.17(18)$ \\
\hline $\mathrm{C}(12)-\mathrm{P}(1)-\mathrm{C}(13)$ & $106.1(2)$ \\
\hline $\mathrm{C}(19)-\mathrm{O}(3)-\mathrm{P}(1)$ & 121.2(4) \\
\hline $\mathrm{C}(1)-\mathrm{N}(1)-\mathrm{S}(1)$ & $114.0(3)$ \\
\hline $\mathrm{C}(1)-\mathrm{N}(1)-\mathrm{H}(1 \mathrm{~N})$ & $122(3)$ \\
\hline $\mathrm{S}(1)-\mathrm{N}(1)-\mathrm{H}(1 \mathrm{~N})$ & $102(3)$ \\
\hline $\mathrm{N}(1)-\mathrm{C}(1)-\mathrm{C}(2)$ & 115.1(4) \\
\hline $\mathrm{N}(1)-\mathrm{C}(1)-\mathrm{C}(12)$ & $107.3(3)$ \\
\hline $\mathrm{C}(2)-\mathrm{C}(1)-\mathrm{C}(12)$ & $108.0(3)$ \\
\hline $\mathrm{N}(1)-\mathrm{C}(1)-\mathrm{H}(1)$ & 108.8 \\
\hline $\mathrm{C}(2)-\mathrm{C}(1)-\mathrm{H}(1)$ & 108.8 \\
\hline $\mathrm{C}(12)-\mathrm{C}(1)-\mathrm{H}(1)$ & 108.8 \\
\hline $\mathrm{C}(3)-\mathrm{C}(2)-\mathrm{C}(11)$ & $119.0(4)$ \\
\hline $\mathrm{C}(3)-\mathrm{C}(2)-\mathrm{C}(1)$ & $119.9(4)$ \\
\hline $\mathrm{C}(11)-\mathrm{C}(2)-\mathrm{C}(1)$ & $121.1(4)$ \\
\hline $\mathrm{C}(2)-\mathrm{C}(3)-\mathrm{C}(4)$ & $121.7(4)$ \\
\hline $\mathrm{C}(2)-\mathrm{C}(3)-\mathrm{H}(3)$ & 119.2 \\
\hline $\mathrm{C}(4)-\mathrm{C}(3)-\mathrm{H}(3)$ & 119.2 \\
\hline $\mathrm{C}(5)-\mathrm{C}(4)-\mathrm{C}(9)$ & $118.0(4)$ \\
\hline$C(5)-C(4)-C(3)$ & $123.6(5)$ \\
\hline $\mathrm{C}(9)-\mathrm{C}(4)-\mathrm{C}(3)$ & $118.3(4)$ \\
\hline$C(6)-C(5)-C(4)$ & $122.3(5)$ \\
\hline $\mathrm{C}(6)-\mathrm{C}(5)-\mathrm{H}(5)$ & 118.9 \\
\hline $\mathrm{C}(4)-\mathrm{C}(5)-\mathrm{H}(5)$ & 118.9 \\
\hline
\end{tabular}




\begin{tabular}{|c|c|}
\hline$C(5)-C(6)-C(7)$ & $119.9(5)$ \\
\hline $\mathrm{C}(5)-\mathrm{C}(6)-\mathrm{H}(6)$ & 120.0 \\
\hline $\mathrm{C}(7)-\mathrm{C}(6)-\mathrm{H}(6)$ & 120.0 \\
\hline$C(8)-C(7)-C(6)$ & $120.1(4)$ \\
\hline $\mathrm{C}(8)-\mathrm{C}(7)-\mathrm{H}(7)$ & 120.0 \\
\hline $\mathrm{C}(6)-\mathrm{C}(7)-\mathrm{H}(7)$ & 120.0 \\
\hline $\mathrm{C}(7)-\mathrm{C}(8)-\mathrm{C}(9)$ & $120.3(5)$ \\
\hline $\mathrm{C}(7)-\mathrm{C}(8)-\mathrm{H}(8)$ & 119.9 \\
\hline $\mathrm{C}(9)-\mathrm{C}(8)-\mathrm{H}(8)$ & 119.9 \\
\hline$C(4)-C(9)-C(10)$ & $118.6(4)$ \\
\hline$C(4)-C(9)-C(8)$ & $119.3(4)$ \\
\hline$C(10)-C(9)-C(8)$ & $122.1(5)$ \\
\hline $\mathrm{C}(11)-\mathrm{C}(10)-\mathrm{C}(9)$ & $121.4(5)$ \\
\hline $\mathrm{C}(11)-\mathrm{C}(10)-\mathrm{H}(10)$ & 119.3 \\
\hline $\mathrm{C}(9)-\mathrm{C}(10)-\mathrm{H}(10)$ & 119.3 \\
\hline$C(10)-C(11)-C(2)$ & $121.0(4)$ \\
\hline $\mathrm{C}(10)-\mathrm{C}(11)-\mathrm{H}(11)$ & 119.5 \\
\hline $\mathrm{C}(2)-\mathrm{C}(11)-\mathrm{H}(11)$ & 119.5 \\
\hline $\mathrm{C}(1)-\mathrm{C}(12)-\mathrm{P}(1)$ & $116.3(3)$ \\
\hline $\mathrm{C}(1)-\mathrm{C}(12)-\mathrm{H}(12 \mathrm{~A})$ & 108.2 \\
\hline $\mathrm{P}(1)-\mathrm{C}(12)-\mathrm{H}(12 \mathrm{~A})$ & 108.2 \\
\hline $\mathrm{C}(1)-\mathrm{C}(12)-\mathrm{H}(12 \mathrm{~B})$ & 108.2 \\
\hline $\mathrm{P}(1)-\mathrm{C}(12)-\mathrm{H}(12 \mathrm{~B})$ & 108.2 \\
\hline $\mathrm{H}(12 \mathrm{~A})-\mathrm{C}(12)-\mathrm{H}(12 \mathrm{~B})$ & 107.4 \\
\hline$C(14)-C(13)-C(18)$ & $119.3(4)$ \\
\hline $\mathrm{C}(14)-\mathrm{C}(13)-\mathrm{P}(1)$ & $120.0(3)$ \\
\hline $\mathrm{C}(18)-\mathrm{C}(13)-\mathrm{P}(1)$ & $120.8(3)$ \\
\hline$C(15)-C(14)-C(13)$ & $120.2(5)$ \\
\hline $\mathrm{C}(15)-\mathrm{C}(14)-\mathrm{H}(14)$ & 119.9 \\
\hline $\mathrm{C}(13)-\mathrm{C}(14)-\mathrm{H}(14)$ & 119.9 \\
\hline $\mathrm{C}(14)-\mathrm{C}(15)-\mathrm{C}(16)$ & $120.5(5)$ \\
\hline $\mathrm{C}(14)-\mathrm{C}(15)-\mathrm{H}(15)$ & 119.8 \\
\hline $\mathrm{C}(16)-\mathrm{C}(15)-\mathrm{H}(15)$ & 119.8 \\
\hline$C(15)-C(16)-C(17)$ & $120.1(5)$ \\
\hline $\mathrm{C}(15)-\mathrm{C}(16)-\mathrm{H}(16)$ & 119.9 \\
\hline$C(17)-C(16)-H(16)$ & 119.9 \\
\hline$C(18)-C(17)-C(16)$ & $120.0(5)$ \\
\hline $\mathrm{C}(18)-\mathrm{C}(17)-\mathrm{H}(17)$ & 120.0 \\
\hline
\end{tabular}




\begin{tabular}{|c|c|}
\hline $\mathrm{C}(16)-\mathrm{C}(17)-\mathrm{H}(17)$ & 120.0 \\
\hline $\mathrm{C}(17)-\mathrm{C}(18)-\mathrm{C}(13)$ & $119.9(5)$ \\
\hline $\mathrm{C}(17)-\mathrm{C}(18)-\mathrm{H}(18)$ & 120.1 \\
\hline $\mathrm{C}(13)-\mathrm{C}(18)-\mathrm{H}(18)$ & 120.1 \\
\hline $\mathrm{O}(3)-\mathrm{C}(19)-\mathrm{H}(19 \mathrm{~A})$ & 109.5 \\
\hline $\mathrm{O}(3)-\mathrm{C}(19)-\mathrm{H}(19 \mathrm{~B})$ & 109.5 \\
\hline$H(19 A)-C(19)-H(19 B)$ & 109.5 \\
\hline $\mathrm{O}(3)-\mathrm{C}(19)-\mathrm{H}(19 \mathrm{C})$ & 109.5 \\
\hline $\mathrm{H}(19 \mathrm{~A})-\mathrm{C}(19)-\mathrm{H}(19 \mathrm{C})$ & 109.5 \\
\hline $\mathrm{H}(19 \mathrm{~B})-\mathrm{C}(19)-\mathrm{H}(19 \mathrm{C})$ & 109.5 \\
\hline$C(21)-C(20)-C(23)$ & $108.4(6)$ \\
\hline $\mathrm{C}(21)-\mathrm{C}(20)-\mathrm{C}(22)$ & $111.9(6)$ \\
\hline$C(23)-C(20)-C(22)$ & $111.4(6)$ \\
\hline$C(21)-C(20)-S(1)$ & $107.3(4)$ \\
\hline$C(23)-C(20)-S(1)$ & $106.4(4)$ \\
\hline$C(22)-C(20)-S(1)$ & $111.2(4)$ \\
\hline $\mathrm{C}(20)-\mathrm{C}(21)-\mathrm{H}(21 \mathrm{~A})$ & 109.5 \\
\hline $\mathrm{C}(20)-\mathrm{C}(21)-\mathrm{H}(21 \mathrm{~B})$ & 109.5 \\
\hline $\mathrm{H}(21 \mathrm{~A})-\mathrm{C}(21)-\mathrm{H}(21 \mathrm{~B})$ & 109.5 \\
\hline $\mathrm{C}(20)-\mathrm{C}(21)-\mathrm{H}(21 \mathrm{C})$ & 109.5 \\
\hline $\mathrm{H}(21 \mathrm{~A})-\mathrm{C}(21)-\mathrm{H}(21 \mathrm{C})$ & 109.5 \\
\hline $\mathrm{H}(21 \mathrm{~B})-\mathrm{C}(21)-\mathrm{H}(21 \mathrm{C})$ & 109.5 \\
\hline $\mathrm{C}(20)-\mathrm{C}(22)-\mathrm{H}(22 \mathrm{~A})$ & 109.5 \\
\hline $\mathrm{C}(20)-\mathrm{C}(22)-\mathrm{H}(22 \mathrm{~B})$ & 109.5 \\
\hline $\mathrm{H}(22 \mathrm{~A})-\mathrm{C}(22)-\mathrm{H}(22 \mathrm{~B})$ & 109.5 \\
\hline $\mathrm{C}(20)-\mathrm{C}(22)-\mathrm{H}(22 \mathrm{C})$ & 109.5 \\
\hline $\mathrm{H}(22 \mathrm{~A})-\mathrm{C}(22)-\mathrm{H}(22 \mathrm{C})$ & 109.5 \\
\hline $\mathrm{H}(22 \mathrm{~B})-\mathrm{C}(22)-\mathrm{H}(22 \mathrm{C})$ & 109.5 \\
\hline $\mathrm{C}(20)-\mathrm{C}(23)-\mathrm{H}(23 \mathrm{~A})$ & 109.5 \\
\hline $\mathrm{C}(20)-\mathrm{C}(23)-\mathrm{H}(23 \mathrm{~B})$ & 109.5 \\
\hline $\mathrm{H}(23 \mathrm{~A})-\mathrm{C}(23)-\mathrm{H}(23 \mathrm{~B})$ & 109.5 \\
\hline $\mathrm{C}(20)-\mathrm{C}(23)-\mathrm{H}(23 \mathrm{C})$ & 109.5 \\
\hline $\mathrm{H}(23 \mathrm{~A})-\mathrm{C}(23)-\mathrm{H}(23 \mathrm{C})$ & 109.5 \\
\hline $\mathrm{H}(23 \mathrm{~B})-\mathrm{C}(23)-\mathrm{H}(23 \mathrm{C})$ & 109.5 \\
\hline $\mathrm{O}(4)-\mathrm{S}(2)-\mathrm{N}(2)$ & $113.1(2)$ \\
\hline $\mathrm{O}(4)-\mathrm{S}(2)-\mathrm{C}(43)$ & $105.4(2)$ \\
\hline $\mathrm{N}(2)-\mathrm{S}(2)-\mathrm{C}(43)$ & $98.07(19)$ \\
\hline $\mathrm{O}(5)-\mathrm{P}(2)-\mathrm{O}(6)$ & $115.73(19)$ \\
\hline
\end{tabular}




\begin{tabular}{|c|c|}
\hline $\mathrm{O}(5)-\mathrm{P}(2)-\mathrm{C}(35)$ & $114.2(2)$ \\
\hline $\mathrm{O}(6)-\mathrm{P}(2)-\mathrm{C}(35)$ & $103.4(2)$ \\
\hline $\mathrm{O}(5)-\mathrm{P}(2)-\mathrm{C}(36)$ & $110.8(2)$ \\
\hline $\mathrm{O}(6)-\mathrm{P}(2)-\mathrm{C}(36)$ & $106.02(18)$ \\
\hline $\mathrm{C}(35)-\mathrm{P}(2)-\mathrm{C}(36)$ & $105.79(19)$ \\
\hline $\mathrm{C}(42)-\mathrm{O}(6)-\mathrm{P}(2)$ & $118.7(3)$ \\
\hline $\mathrm{C}(24)-\mathrm{N}(2)-\mathrm{S}(2)$ & $118.2(3)$ \\
\hline $\mathrm{C}(24)-\mathrm{N}(2)-\mathrm{H}(2 \mathrm{~N})$ & $110(3)$ \\
\hline $\mathrm{S}(2)-\mathrm{N}(2)-\mathrm{H}(2 \mathrm{~N})$ & $118(3)$ \\
\hline $\mathrm{N}(2)-\mathrm{C}(24)-\mathrm{C}(25)$ & $115.3(4)$ \\
\hline $\mathrm{N}(2)-\mathrm{C}(24)-\mathrm{C}(35)$ & $108.9(3)$ \\
\hline $\mathrm{C}(25)-\mathrm{C}(24)-\mathrm{C}(35)$ & 109.3(3) \\
\hline $\mathrm{N}(2)-\mathrm{C}(24)-\mathrm{H}(24)$ & 107.7 \\
\hline $\mathrm{C}(25)-\mathrm{C}(24)-\mathrm{H}(24)$ & 107.7 \\
\hline $\mathrm{C}(35)-\mathrm{C}(24)-\mathrm{H}(24)$ & 107.7 \\
\hline$C(26)-C(25)-C(34)$ & $119.7(4)$ \\
\hline $\mathrm{C}(26)-\mathrm{C}(25)-\mathrm{C}(24)$ & $119.4(4)$ \\
\hline $\mathrm{C}(34)-\mathrm{C}(25)-\mathrm{C}(24)$ & $120.7(4)$ \\
\hline $\mathrm{C}(25)-\mathrm{C}(26)-\mathrm{C}(27)$ & $119.9(5)$ \\
\hline $\mathrm{C}(25)-\mathrm{C}(26)-\mathrm{H}(26)$ & 120.1 \\
\hline $\mathrm{C}(27)-\mathrm{C}(26)-\mathrm{H}(26)$ & 120.1 \\
\hline $\mathrm{C}(32)-\mathrm{C}(27)-\mathrm{C}(26)$ & 119.7(4) \\
\hline $\mathrm{C}(32)-\mathrm{C}(27)-\mathrm{C}(28)$ & $119.4(4)$ \\
\hline $\mathrm{C}(26)-\mathrm{C}(27)-\mathrm{C}(28)$ & $120.9(5)$ \\
\hline $\mathrm{C}(29)-\mathrm{C}(28)-\mathrm{C}(27)$ & $119.4(6)$ \\
\hline $\mathrm{C}(29)-\mathrm{C}(28)-\mathrm{H}(28)$ & 120.3 \\
\hline $\mathrm{C}(27)-\mathrm{C}(28)-\mathrm{H}(28)$ & 120.3 \\
\hline $\mathrm{C}(30)-\mathrm{C}(29)-\mathrm{C}(28)$ & $120.7(6)$ \\
\hline $\mathrm{C}(30)-\mathrm{C}(29)-\mathrm{H}(29)$ & 119.6 \\
\hline $\mathrm{C}(28)-\mathrm{C}(29)-\mathrm{H}(29)$ & 119.6 \\
\hline $\mathrm{C}(31)-\mathrm{C}(30)-\mathrm{C}(29)$ & $122.4(5)$ \\
\hline $\mathrm{C}(31)-\mathrm{C}(30)-\mathrm{H}(30)$ & 118.8 \\
\hline $\mathrm{C}(29)-\mathrm{C}(30)-\mathrm{H}(30)$ & 118.8 \\
\hline $\mathrm{C}(30)-\mathrm{C}(31)-\mathrm{C}(32)$ & $118.9(6)$ \\
\hline $\mathrm{C}(30)-\mathrm{C}(31)-\mathrm{H}(31)$ & 120.6 \\
\hline $\mathrm{C}(32)-\mathrm{C}(31)-\mathrm{H}(31)$ & 120.6 \\
\hline $\mathrm{C}(27)-\mathrm{C}(32)-\mathrm{C}(33)$ & $119.4(4)$ \\
\hline $\mathrm{C}(27)-\mathrm{C}(32)-\mathrm{C}(31)$ & $119.3(5)$ \\
\hline
\end{tabular}




\begin{tabular}{|c|c|}
\hline$C(33)-C(32)-C(31)$ & $121.4(6)$ \\
\hline $\mathrm{C}(34)-\mathrm{C}(33)-\mathrm{C}(32)$ & $120.2(5)$ \\
\hline $\mathrm{C}(34)-\mathrm{C}(33)-\mathrm{H}(33)$ & 119.9 \\
\hline $\mathrm{C}(32)-\mathrm{C}(33)-\mathrm{H}(33)$ & 119.9 \\
\hline$C(33)-C(34)-C(25)$ & $121.1(5)$ \\
\hline $\mathrm{C}(33)-\mathrm{C}(34)-\mathrm{H}(34)$ & 119.4 \\
\hline $\mathrm{C}(25)-\mathrm{C}(34)-\mathrm{H}(34)$ & 119.4 \\
\hline $\mathrm{C}(24)-\mathrm{C}(35)-\mathrm{P}(2)$ & $116.3(3)$ \\
\hline $\mathrm{C}(24)-\mathrm{C}(35)-\mathrm{H}(35 \mathrm{~A})$ & 108.2 \\
\hline $\mathrm{P}(2)-\mathrm{C}(35)-\mathrm{H}(35 \mathrm{~A})$ & 108.2 \\
\hline $\mathrm{C}(24)-\mathrm{C}(35)-\mathrm{H}(35 \mathrm{~B})$ & 108.2 \\
\hline $\mathrm{P}(2)-\mathrm{C}(35)-\mathrm{H}(35 \mathrm{~B})$ & 108.2 \\
\hline $\mathrm{H}(35 \mathrm{~A})-\mathrm{C}(35)-\mathrm{H}(35 \mathrm{~B})$ & 107.4 \\
\hline $\mathrm{C}(41)-\mathrm{C}(36)-\mathrm{C}(37)$ & $119.0(4)$ \\
\hline $\mathrm{C}(41)-\mathrm{C}(36)-\mathrm{P}(2)$ & $121.4(4)$ \\
\hline $\mathrm{C}(37)-\mathrm{C}(36)-\mathrm{P}(2)$ & $119.5(4)$ \\
\hline $\mathrm{C}(36)-\mathrm{C}(37)-\mathrm{C}(38)$ & $119.7(5)$ \\
\hline $\mathrm{C}(36)-\mathrm{C}(37)-\mathrm{H}(37)$ & 120.2 \\
\hline $\mathrm{C}(38)-\mathrm{C}(37)-\mathrm{H}(37)$ & 120.2 \\
\hline $\mathrm{C}(39)-\mathrm{C}(38)-\mathrm{C}(37)$ & $120.1(6)$ \\
\hline $\mathrm{C}(39)-\mathrm{C}(38)-\mathrm{H}(38)$ & 119.9 \\
\hline $\mathrm{C}(37)-\mathrm{C}(38)-\mathrm{H}(38)$ & 119.9 \\
\hline $\mathrm{C}(40)-\mathrm{C}(39)-\mathrm{C}(38)$ & $120.6(5)$ \\
\hline $\mathrm{C}(40)-\mathrm{C}(39)-\mathrm{H}(39)$ & 119.7 \\
\hline $\mathrm{C}(38)-\mathrm{C}(39)-\mathrm{H}(39)$ & 119.7 \\
\hline$C(39)-C(40)-C(41)$ & $120.0(6)$ \\
\hline $\mathrm{C}(39)-\mathrm{C}(40)-\mathrm{H}(40)$ & 120.0 \\
\hline $\mathrm{C}(41)-\mathrm{C}(40)-\mathrm{H}(40)$ & 120.0 \\
\hline$C(36)-C(41)-C(40)$ & $120.6(5)$ \\
\hline $\mathrm{C}(36)-\mathrm{C}(41)-\mathrm{H}(41)$ & 119.7 \\
\hline $\mathrm{C}(40)-\mathrm{C}(41)-\mathrm{H}(41)$ & 119.7 \\
\hline $\mathrm{O}(6)-\mathrm{C}(42)-\mathrm{H}(42 \mathrm{~A})$ & 109.5 \\
\hline $\mathrm{O}(6)-\mathrm{C}(42)-\mathrm{H}(42 \mathrm{~B})$ & 109.5 \\
\hline $\mathrm{H}(42 \mathrm{~A})-\mathrm{C}(42)-\mathrm{H}(42 \mathrm{~B})$ & 109.5 \\
\hline $\mathrm{O}(6)-\mathrm{C}(42)-\mathrm{H}(42 \mathrm{C})$ & 109.5 \\
\hline $\mathrm{H}(42 \mathrm{~A})-\mathrm{C}(42)-\mathrm{H}(42 \mathrm{C})$ & 109.5 \\
\hline $\mathrm{H}(42 \mathrm{~B})-\mathrm{C}(42)-\mathrm{H}(42 \mathrm{C})$ & 109.5 \\
\hline$C(45)-C(43)-C(44)$ & $112.0(4)$ \\
\hline
\end{tabular}




\begin{tabular}{ll}
$\mathrm{C}(45)-\mathrm{C}(43)-\mathrm{C}(46)$ & $110.5(4)$ \\
$\mathrm{C}(44)-\mathrm{C}(43)-\mathrm{C}(46)$ & $110.5(4)$ \\
$\mathrm{C}(45)-\mathrm{C}(43)-\mathrm{S}(2)$ & $110.7(3)$ \\
$\mathrm{C}(44)-\mathrm{C}(43)-\mathrm{S}(2)$ & $109.5(3)$ \\
$\mathrm{C}(46)-\mathrm{C}(43)-\mathrm{S}(2)$ & $103.3(3)$ \\
$\mathrm{C}(43)-\mathrm{C}(44)-\mathrm{H}(44 \mathrm{~A})$ & 109.5 \\
$\mathrm{C}(43)-\mathrm{C}(44)-\mathrm{H}(44 \mathrm{~B})$ & 109.5 \\
$\mathrm{H}(44 \mathrm{~A})-\mathrm{C}(44)-\mathrm{H}(44 \mathrm{~B})$ & 109.5 \\
$\mathrm{C}(43)-\mathrm{C}(44)-\mathrm{H}(44 \mathrm{C})$ & 109.5 \\
$\mathrm{H}(44 \mathrm{~A})-\mathrm{C}(44)-\mathrm{H}(44 \mathrm{C})$ & 109.5 \\
$\mathrm{H}(44 \mathrm{~B})-\mathrm{C}(44)-\mathrm{H}(44 \mathrm{C})$ & 109.5 \\
$\mathrm{C}(43)-\mathrm{C}(45)-\mathrm{H}(45 \mathrm{~A})$ & 109.5 \\
$\mathrm{C}(43)-\mathrm{C}(45)-\mathrm{H}(45 \mathrm{~B})$ & 109.5 \\
$\mathrm{H}(45 \mathrm{~A})-\mathrm{C}(45)-\mathrm{H}(45 \mathrm{~B})$ & 109.5 \\
$\mathrm{C}(43)-\mathrm{C}(45)-\mathrm{H}(45 \mathrm{C})$ & 109.5 \\
$\mathrm{H}(45 \mathrm{~A})-\mathrm{C}(45)-\mathrm{H}(45 \mathrm{C})$ & 109.5 \\
$\mathrm{H}(45 \mathrm{~B})-\mathrm{C}(45)-\mathrm{H}(45 \mathrm{C})$ & 109.5 \\
$\mathrm{C}(43)-\mathrm{C}(46)-\mathrm{H}(46 \mathrm{~A})$ & 109.5 \\
$\mathrm{C}(43)-\mathrm{C}(46)-\mathrm{H}(46 \mathrm{~B})$ & 109.5 \\
$\mathrm{H}(46 \mathrm{~A})-\mathrm{C}(46)-\mathrm{H}(46 \mathrm{~B})$ & 109.5 \\
$\mathrm{C}(43)-\mathrm{C}(46)-\mathrm{H}(46 \mathrm{C})$ & 109.5 \\
$\mathrm{H}(46 \mathrm{~A})-\mathrm{C}(46)-\mathrm{H}(46 \mathrm{C})$ & 109.5 \\
$\mathrm{H}(46 \mathrm{~B})-\mathrm{C}(46)-\mathrm{H}(46 \mathrm{C})$ & 109.5 \\
& \\
\hline
\end{tabular}

Symmetry transformations used to generate equivalent atoms:

Table 4. Anisotropic displacement parameters $\left(\AA^{2} \times 10^{3}\right)$ for compound $\mathbf{1 0}(\boldsymbol{R}, \boldsymbol{S}, \boldsymbol{S})$. The anisotropic displacement factor exponent takes the form: $-2 \pi^{2}\left[\mathrm{~h}^{2} \mathrm{a}^{* 2} \mathrm{U}^{11}+\ldots+2 \mathrm{~h} \mathrm{k}\right.$ a* $b^{*} \mathrm{U}^{12}$ ]

\begin{tabular}{lcccccc}
\hline & $\mathrm{U}^{11}$ & $\mathrm{U}^{22}$ & $\mathrm{U}^{33}$ & $\mathrm{U}^{23}$ & $\mathrm{U}^{13}$ & $\mathrm{U}^{12}$ \\
\hline $\mathrm{S}(1)$ & $39(1)$ & $101(1)$ & $45(1)$ & $33(1)$ & $27(1)$ & $29(1)$ \\
$\mathrm{P}(1)$ & $32(1)$ & $28(1)$ & $26(1)$ & $-6(1)$ & $12(1)$ & $-1(1)$ \\
$\mathrm{O}(1)$ & $114(4)$ & $204(7)$ & $51(2)$ & $4(3)$ & $32(2)$ & $111(5)$ \\
$\mathrm{O}(2)$ & $48(2)$ & $38(2)$ & $57(2)$ & $-5(2)$ & $25(2)$ & $-12(2)$ \\
$\mathrm{O}(3)$ & $40(2)$ & $43(2)$ & $23(1)$ & $-5(2)$ & $3(1)$ & $6(2)$ \\
$\mathrm{N}(1)$ & $29(2)$ & $36(2)$ & $31(2)$ & $1(2)$ & $14(2)$ & $4(2)$
\end{tabular}




\begin{tabular}{|c|c|c|c|c|c|c|}
\hline $\mathrm{C}(1)$ & $29(2)$ & $37(3)$ & $29(2)$ & $-6(2)$ & $14(2)$ & $1(2)$ \\
\hline $\mathrm{C}(2)$ & $28(2)$ & $38(3)$ & $28(2)$ & $2(2)$ & $11(2)$ & $5(2)$ \\
\hline $\mathrm{C}(3)$ & $31(2)$ & $26(3)$ & $31(2)$ & $0(2)$ & $7(2)$ & $6(2)$ \\
\hline $\mathrm{C}(4)$ & $30(2)$ & $32(3)$ & $30(2)$ & $5(2)$ & $13(2)$ & $9(2)$ \\
\hline $\mathrm{C}(5)$ & $42(2)$ & $36(3)$ & $49(3)$ & $8(3)$ & $19(2)$ & $1(2)$ \\
\hline$C(6)$ & $49(3)$ & $52(4)$ & $33(2)$ & $8(3)$ & $17(2)$ & $3(3)$ \\
\hline $\mathrm{C}(7)$ & $43(2)$ & $56(4)$ & $26(2)$ & $2(2)$ & $14(2)$ & $7(3)$ \\
\hline $\mathrm{C}(8)$ & $29(2)$ & $41(3)$ & $37(2)$ & $-7(2)$ & $8(2)$ & $6(2)$ \\
\hline $\mathrm{C}(9)$ & $28(2)$ & $34(3)$ & $32(2)$ & $5(2)$ & $12(2)$ & $10(2)$ \\
\hline$C(10)$ & $29(2)$ & $30(3)$ & $40(2)$ & $-3(2)$ & $14(2)$ & $3(2)$ \\
\hline$C(11)$ & $34(2)$ & $36(3)$ & $32(2)$ & $5(2)$ & $17(2)$ & $5(2)$ \\
\hline $\mathrm{C}(12)$ & $29(2)$ & $40(3)$ & $30(2)$ & $-12(2)$ & $11(2)$ & $2(2)$ \\
\hline$C(13)$ & $33(2)$ & $23(2)$ & $26(2)$ & $-3(2)$ & $18(2)$ & $1(2)$ \\
\hline$C(14)$ & $48(3)$ & $27(3)$ & $43(3)$ & $-1(2)$ & $19(2)$ & $2(2)$ \\
\hline$C(15)$ & $51(3)$ & $50(4)$ & $50(3)$ & $-5(3)$ & $17(2)$ & $22(3)$ \\
\hline$C(16)$ & $37(2)$ & $73(5)$ & $44(3)$ & $-9(3)$ & $20(2)$ & $11(3)$ \\
\hline $\mathrm{C}(17)$ & $46(3)$ & $51(4)$ & $42(3)$ & $-4(2)$ & $26(2)$ & $-11(3)$ \\
\hline $\mathrm{C}(18)$ & $46(2)$ & $31(3)$ & $31(2)$ & $4(2)$ & $20(2)$ & $0(2)$ \\
\hline$C(19)$ & $69(3)$ & 71(4) & $31(2)$ & $-12(3)$ & $17(2)$ & $-12(3)$ \\
\hline$C(20)$ & $29(2)$ & $44(3)$ & $42(2)$ & $13(2)$ & $10(2)$ & $8(2)$ \\
\hline$C(21)$ & $69(4)$ & $95(6)$ & $74(4)$ & $-27(5)$ & $-13(3)$ & $16(4)$ \\
\hline$C(22)$ & $90(5)$ & $83(6)$ & $77(5)$ & $40(4)$ & $-4(4)$ & $-31(5)$ \\
\hline$C(23)$ & $45(3)$ & $138(8)$ & $98(5)$ & $42(5)$ & $28(3)$ & $42(5)$ \\
\hline$S(2)$ & $29(1)$ & $29(1)$ & $29(1)$ & $-2(1)$ & $10(1)$ & $1(1)$ \\
\hline $\mathrm{P}(2)$ & $25(1)$ & $26(1)$ & $26(1)$ & $-1(1)$ & $10(1)$ & $2(1)$ \\
\hline $\mathrm{O}(4)$ & $32(2)$ & $77(3)$ & $48(2)$ & $15(2)$ & $10(1)$ & $16(2)$ \\
\hline $\mathrm{O}(5)$ & $37(2)$ & $32(2)$ & $44(2)$ & $-10(2)$ & $14(1)$ & $0(2)$ \\
\hline $\mathrm{O}(6)$ & $33(2)$ & $38(2)$ & $35(2)$ & $8(2)$ & $17(1)$ & $8(1)$ \\
\hline $\mathrm{N}(2)$ & $36(2)$ & $28(2)$ & $31(2)$ & $0(2)$ & $18(2)$ & $1(2)$ \\
\hline$C(24)$ & $27(2)$ & $27(2)$ & $29(2)$ & $1(2)$ & $12(2)$ & $2(2)$ \\
\hline$C(25)$ & $28(2)$ & $29(3)$ & $29(2)$ & $3(2)$ & $13(2)$ & $5(2)$ \\
\hline$C(26)$ & $28(2)$ & $43(3)$ & $23(2)$ & $-2(2)$ & $8(2)$ & $2(2)$ \\
\hline $\mathrm{C}(27)$ & $22(2)$ & $65(4)$ & $25(2)$ & $7(2)$ & $9(2)$ & $5(2)$ \\
\hline$C(28)$ & $30(2)$ & $91(5)$ & $34(2)$ & $-16(3)$ & $10(2)$ & $-13(3)$ \\
\hline $\mathrm{C}(29)$ & $25(2)$ & $159(8)$ & $39(3)$ & $-32(4)$ & $13(2)$ & $-22(3)$ \\
\hline$C(30)$ & $34(3)$ & $152(8)$ & $55(3)$ & $-39(4)$ & $22(2)$ & $6(4)$ \\
\hline $\mathrm{C}(31)$ & $35(3)$ & $100(6)$ & $55(3)$ & $-26(3)$ & $22(2)$ & $10(3)$ \\
\hline $\mathrm{C}(32)$ & $33(2)$ & $64(4)$ & $36(2)$ & $-4(3)$ & $18(2)$ & $10(3)$ \\
\hline
\end{tabular}




\begin{tabular}{lllllll}
$\mathrm{C}(33)$ & $56(3)$ & $44(3)$ & $55(3)$ & $-9(3)$ & $32(3)$ & $6(3)$ \\
$\mathrm{C}(34)$ & $37(2)$ & $33(3)$ & $49(3)$ & $0(2)$ & $27(2)$ & $3(2)$ \\
$\mathrm{C}(35)$ & $22(2)$ & $35(3)$ & $22(2)$ & $-5(2)$ & $7(2)$ & $2(2)$ \\
$\mathrm{C}(36)$ & $26(2)$ & $29(3)$ & $30(2)$ & $-1(2)$ & $8(2)$ & $5(2)$ \\
$\mathrm{C}(37)$ & $63(3)$ & $38(3)$ & $37(3)$ & $2(2)$ & $18(2)$ & $3(3)$ \\
$\mathrm{C}(38)$ & $106(5)$ & $42(4)$ & $40(3)$ & $14(3)$ & $31(3)$ & $17(4)$ \\
$\mathrm{C}(39)$ & $55(3)$ & $81(5)$ & $32(3)$ & $7(3)$ & $8(2)$ & $26(3)$ \\
$\mathrm{C}(40)$ & $41(3)$ & $75(5)$ & $42(3)$ & $-12(3)$ & $7(2)$ & $-3(3)$ \\
$\mathrm{C}(41)$ & $39(2)$ & $49(4)$ & $39(3)$ & $-4(2)$ & $13(2)$ & $-3(2)$ \\
$\mathrm{C}(42)$ & $31(2)$ & $73(4)$ & $51(3)$ & $2(3)$ & $24(2)$ & $13(3)$ \\
$\mathrm{C}(43)$ & $36(2)$ & $32(3)$ & $28(2)$ & $1(2)$ & $15(2)$ & $5(2)$ \\
$\mathrm{C}(44)$ & $38(2)$ & $53(3)$ & $48(3)$ & $5(3)$ & $22(2)$ & $4(2)$ \\
$\mathrm{C}(45)$ & $67(3)$ & $32(3)$ & $42(3)$ & $1(2)$ & $23(2)$ & $0(3)$ \\
$\mathrm{C}(46)$ & $57(3)$ & $55(4)$ & $30(2)$ & $-3(2)$ & $20(2)$ & $-7(3)$ \\
\hline
\end{tabular}

Table 5. Hydrogen coordinates $\left(\times 10^{4}\right)$ and isotropic displacement parameters $\left(\AA^{2} \times 10^{3}\right)$ for compound $10(R, S, S)$.

\begin{tabular}{|c|c|c|c|c|}
\hline & $\mathrm{x}$ & $\mathrm{y}$ & $\mathrm{z}$ & $\mathrm{U}(\mathrm{eq})$ \\
\hline $\mathrm{H}(1 \mathrm{~N})$ & $8089(13)$ & $6560(40)$ & $8770(20)$ & 38 \\
\hline $\mathrm{H}(1)$ & 8064 & 10963 & 8310 & 37 \\
\hline $\mathrm{H}(3)$ & 8090 & 11342 & 7407 & 38 \\
\hline $\mathrm{H}(5)$ & 8134 & 11911 & 6425 & 51 \\
\hline $\mathrm{H}(6)$ & 8044 & 10611 & 5515 & 53 \\
\hline $\mathrm{H}(7)$ & 7715 & 7107 & 5164 & 50 \\
\hline $\mathrm{H}(8)$ & 7511 & 4930 & 5760 & 45 \\
\hline $\mathrm{H}(10)$ & 7490 & 4303 & 6749 & 40 \\
\hline $\mathrm{H}(11)$ & 7624 & 5495 & 7684 & 39 \\
\hline $\mathrm{H}(12 \mathrm{~A})$ & 7415 & 8290 & 8350 & 40 \\
\hline $\mathrm{H}(12 \mathrm{~B})$ & 7303 & 10186 & 7857 & 40 \\
\hline $\mathrm{H}(14)$ & 6833 & 15096 & 8302 & 47 \\
\hline $\mathrm{H}(15)$ & 6159 & 15288 & 8160 & 62 \\
\hline $\mathrm{H}(16)$ & 5855 & 12247 & 8404 & 60 \\
\hline $\mathrm{H}(17)$ & 6224 & 8945 & 8781 & 53 \\
\hline $\mathrm{H}(18)$ & 6907 & 8747 & 8948 & 42 \\
\hline
\end{tabular}




\begin{tabular}{|c|c|c|c|c|}
\hline $\mathrm{H}(19 \mathrm{~A})$ & 7944 & 13054 & 9955 & 87 \\
\hline $\mathrm{H}(19 \mathrm{~B})$ & 7958 & 10690 & 10266 & 87 \\
\hline $\mathrm{H}(19 \mathrm{C})$ & 7541 & 12045 & 9972 & 87 \\
\hline $\mathrm{H}(21 \mathrm{~A})$ & 9030 & 8138 & 10374 & 141 \\
\hline $\mathrm{H}(21 \mathrm{~B})$ & 8590 & 8829 & 9895 & 141 \\
\hline $\mathrm{H}(21 \mathrm{C})$ & 8985 & 9828 & 9852 & 141 \\
\hline $\mathrm{H}(22 \mathrm{~A})$ & 8801 & 3927 & 10064 & 145 \\
\hline $\mathrm{H}(22 \mathrm{~B})$ & 8624 & 3394 & 9373 & 145 \\
\hline $\mathrm{H}(22 \mathrm{C})$ & 8380 & 5008 & 9623 & 145 \\
\hline $\mathrm{H}(23 \mathrm{~A})$ & 9430 & 7180 & 9574 & 141 \\
\hline $\mathrm{H}(23 \mathrm{~B})$ & 9322 & 4566 & 9528 & 141 \\
\hline $\mathrm{H}(23 \mathrm{C})$ & 9464 & 5833 & 10147 & 141 \\
\hline $\mathrm{H}(2 \mathrm{~N})$ & $4838(13)$ & $5560(40)$ & $7444(18)$ & 36 \\
\hline $\mathrm{H}(24)$ & 5095 & 9824 & 7351 & 33 \\
\hline $\mathrm{H}(26)$ & 5726 & 10000 & 7336 & 39 \\
\hline $\mathrm{H}(28)$ & 6387 & 10500 & 7247 & 63 \\
\hline $\mathrm{H}(29)$ & 6850 & 9166 & 6891 & 90 \\
\hline $\mathrm{H}(30)$ & 6757 & 5729 & 6428 & 95 \\
\hline $\mathrm{H}(31)$ & 6212 & 3447 & 6304 & 75 \\
\hline $\mathrm{H}(33)$ & 5556 & 2908 & 6428 & 58 \\
\hline $\mathrm{H}(34)$ & 5068 & 4306 & 6704 & 44 \\
\hline $\mathrm{H}(35 \mathrm{~A})$ & 4473 & 7236 & 6467 & 33 \\
\hline $\mathrm{H}(35 \mathrm{~B})$ & 4712 & 9055 & 6267 & 33 \\
\hline $\mathrm{H}(37)$ & 4073 & 14245 & 5694 & 56 \\
\hline $\mathrm{H}(38)$ & 3615 & 14526 & 4687 & 75 \\
\hline $\mathrm{H}(39)$ & 3182 & 11552 & 4224 & 72 \\
\hline $\mathrm{H}(40)$ & 3186 & 8342 & 4743 & 68 \\
\hline $\mathrm{H}(41)$ & 3639 & 8015 & 5749 & 52 \\
\hline $\mathrm{H}(42 \mathrm{~A})$ & 3914 & 12311 & 7315 & 74 \\
\hline $\mathrm{H}(42 \mathrm{~B})$ & 3669 & 10103 & 7330 & 74 \\
\hline $\mathrm{H}(42 \mathrm{C})$ & 3540 & 11493 & 6730 & 74 \\
\hline $\mathrm{H}(44 \mathrm{~A})$ & 4252 & 6334 & 7804 & 68 \\
\hline $\mathrm{H}(44 \mathrm{~B})$ & 4365 & 8414 & 8249 & 68 \\
\hline $\mathrm{H}(44 \mathrm{C})$ & 4192 & 6152 & 8402 & 68 \\
\hline $\mathrm{H}(45 \mathrm{~A})$ & 4691 & 2517 & 8660 & 71 \\
\hline $\mathrm{H}(45 \mathrm{~B})$ & 5103 & 2889 & 8587 & 71 \\
\hline $\mathrm{H}(45 \mathrm{C})$ & 4679 & 3002 & 8022 & 71 \\
\hline $\mathrm{H}(46 \mathrm{~A})$ & 4958 & 8038 & 9268 & 70 \\
\hline
\end{tabular}




\begin{tabular}{lllll}
$\mathrm{H}(46 \mathrm{~B})$ & 5248 & 5894 & 9408 & 70 \\
$\mathrm{H}(46 \mathrm{C})$ & 4804 & 5653 & 9386 & 70 \\
& & & \\
\hline
\end{tabular}

Table 6. Torsion angles $\left[{ }^{\circ}\right]$ for compound $10(R, S, S)$.

\begin{tabular}{|c|c|}
\hline $\mathrm{O}(2)-\mathrm{P}(1)-\mathrm{O}(3)-\mathrm{C}(19)$ & $47.5(4)$ \\
\hline $\mathrm{C}(12)-\mathrm{P}(1)-\mathrm{O}(3)-\mathrm{C}(19)$ & $172.6(4)$ \\
\hline $\mathrm{C}(13)-\mathrm{P}(1)-\mathrm{O}(3)-\mathrm{C}(19)$ & $-76.6(4)$ \\
\hline $\mathrm{O}(1)-\mathrm{S}(1)-\mathrm{N}(1)-\mathrm{C}(1)$ & $-90.0(4)$ \\
\hline $\mathrm{C}(20)-\mathrm{S}(1)-\mathrm{N}(1)-\mathrm{C}(1)$ & $160.4(3)$ \\
\hline $\mathrm{S}(1)-\mathrm{N}(1)-\mathrm{C}(1)-\mathrm{C}(2)$ & $61.5(5)$ \\
\hline$S(1)-N(1)-C(1)-C(12)$ & $-178.3(3)$ \\
\hline $\mathrm{N}(1)-\mathrm{C}(1)-\mathrm{C}(2)-\mathrm{C}(3)$ & $-125.0(4)$ \\
\hline$C(12)-C(1)-C(2)-C(3)$ & $115.2(4)$ \\
\hline $\mathrm{N}(1)-\mathrm{C}(1)-\mathrm{C}(2)-\mathrm{C}(11)$ & $54.7(5)$ \\
\hline$C(12)-C(1)-C(2)-C(11)$ & $-65.1(5)$ \\
\hline$C(11)-C(2)-C(3)-C(4)$ & $2.0(6)$ \\
\hline $\mathrm{C}(1)-\mathrm{C}(2)-\mathrm{C}(3)-\mathrm{C}(4)$ & $-178.3(4)$ \\
\hline $\mathrm{C}(2)-\mathrm{C}(3)-\mathrm{C}(4)-\mathrm{C}(5)$ & $-178.4(4)$ \\
\hline $\mathrm{C}(2)-\mathrm{C}(3)-\mathrm{C}(4)-\mathrm{C}(9)$ & $0.3(6)$ \\
\hline$C(9)-C(4)-C(5)-C(6)$ & $-0.8(7)$ \\
\hline$C(3)-C(4)-C(5)-C(6)$ & $177.9(4)$ \\
\hline$C(4)-C(5)-C(6)-C(7)$ & $2.1(7)$ \\
\hline$C(5)-C(6)-C(7)-C(8)$ & $-1.5(7)$ \\
\hline $\mathrm{C}(6)-\mathrm{C}(7)-\mathrm{C}(8)-\mathrm{C}(9)$ & $-0.4(7)$ \\
\hline $\mathrm{C}(5)-\mathrm{C}(4)-\mathrm{C}(9)-\mathrm{C}(10)$ & 177.2(4) \\
\hline$C(3)-C(4)-C(9)-C(10)$ & $-1.6(6)$ \\
\hline$C(5)-C(4)-C(9)-C(8)$ & $-1.1(6)$ \\
\hline $\mathrm{C}(3)-\mathrm{C}(4)-\mathrm{C}(9)-\mathrm{C}(8)$ & $-179.9(4)$ \\
\hline $\mathrm{C}(7)-\mathrm{C}(8)-\mathrm{C}(9)-\mathrm{C}(4)$ & $1.7(6)$ \\
\hline$C(7)-C(8)-C(9)-C(10)$ & $-176.6(4)$ \\
\hline$C(4)-C(9)-C(10)-C(11)$ & $0.6(6)$ \\
\hline$C(8)-C(9)-C(10)-C(11)$ & $178.8(4)$ \\
\hline $\mathrm{C}(9)-\mathrm{C}(10)-\mathrm{C}(11)-\mathrm{C}(2)$ & $1.8(6)$ \\
\hline $\mathrm{C}(3)-\mathrm{C}(2)-\mathrm{C}(11)-\mathrm{C}(10)$ & $-3.0(6)$ \\
\hline$C(1)-C(2)-C(11)-C(10)$ & $177.3(4)$ \\
\hline $\mathrm{N}(1)-\mathrm{C}(1)-\mathrm{C}(12)-\mathrm{P}(1)$ & $81.5(4)$ \\
\hline
\end{tabular}




\begin{tabular}{|c|c|}
\hline $\mathrm{C}(2)-\mathrm{C}(1)-\mathrm{C}(12)-\mathrm{P}(1)$ & $-153.9(3)$ \\
\hline $\mathrm{O}(2)-\mathrm{P}(1)-\mathrm{C}(12)-\mathrm{C}(1)$ & $52.5(4)$ \\
\hline $\mathrm{O}(3)-\mathrm{P}(1)-\mathrm{C}(12)-\mathrm{C}(1)$ & $-73.3(4)$ \\
\hline $\mathrm{C}(13)-\mathrm{P}(1)-\mathrm{C}(12)-\mathrm{C}(1)$ & $175.8(3)$ \\
\hline $\mathrm{O}(2)-\mathrm{P}(1)-\mathrm{C}(13)-\mathrm{C}(14)$ & $13.5(4)$ \\
\hline $\mathrm{O}(3)-\mathrm{P}(1)-\mathrm{C}(13)-\mathrm{C}(14)$ & $140.3(3)$ \\
\hline$C(12)-P(1)-C(13)-C(14)$ & $-112.0(4)$ \\
\hline $\mathrm{O}(2)-\mathrm{P}(1)-\mathrm{C}(13)-\mathrm{C}(18)$ & $-166.8(3)$ \\
\hline $\mathrm{O}(3)-\mathrm{P}(1)-\mathrm{C}(13)-\mathrm{C}(18)$ & $-40.0(4)$ \\
\hline $\mathrm{C}(12)-\mathrm{P}(1)-\mathrm{C}(13)-\mathrm{C}(18)$ & $67.7(4)$ \\
\hline$C(18)-C(13)-C(14)-C(15)$ & $1.4(7)$ \\
\hline$P(1)-C(13)-C(14)-C(15)$ & $-178.9(4)$ \\
\hline $\mathrm{C}(13)-\mathrm{C}(14)-\mathrm{C}(15)-\mathrm{C}(16)$ & $-0.8(8)$ \\
\hline$C(14)-C(15)-C(16)-C(17)$ & $-0.6(8)$ \\
\hline$C(15)-C(16)-C(17)-C(18)$ & $1.4(7)$ \\
\hline$C(16)-C(17)-C(18)-C(13)$ & $-0.8(7)$ \\
\hline$C(14)-C(13)-C(18)-C(17)$ & $-0.6(6)$ \\
\hline$P(1)-C(13)-C(18)-C(17)$ & $179.7(3)$ \\
\hline $\mathrm{O}(1)-\mathrm{S}(1)-\mathrm{C}(20)-\mathrm{C}(21)$ & $179.5(5)$ \\
\hline $\mathrm{N}(1)-\mathrm{S}(1)-\mathrm{C}(20)-\mathrm{C}(21)$ & $-65.9(5)$ \\
\hline $\mathrm{O}(1)-\mathrm{S}(1)-\mathrm{C}(20)-\mathrm{C}(23)$ & $63.6(6)$ \\
\hline $\mathrm{N}(1)-\mathrm{S}(1)-\mathrm{C}(20)-\mathrm{C}(23)$ & $178.2(5)$ \\
\hline $\mathrm{O}(1)-\mathrm{S}(1)-\mathrm{C}(20)-\mathrm{C}(22)$ & $-57.8(6)$ \\
\hline $\mathrm{N}(1)-\mathrm{S}(1)-\mathrm{C}(20)-\mathrm{C}(22)$ & $56.7(5)$ \\
\hline $\mathrm{O}(5)-\mathrm{P}(2)-\mathrm{O}(6)-\mathrm{C}(42)$ & $46.3(4)$ \\
\hline $\mathrm{C}(35)-\mathrm{P}(2)-\mathrm{O}(6)-\mathrm{C}(42)$ & $172.0(3)$ \\
\hline $\mathrm{C}(36)-\mathrm{P}(2)-\mathrm{O}(6)-\mathrm{C}(42)$ & $-77.0(4)$ \\
\hline $\mathrm{O}(4)-\mathrm{S}(2)-\mathrm{N}(2)-\mathrm{C}(24)$ & $-81.6(4)$ \\
\hline $\mathrm{C}(43)-\mathrm{S}(2)-\mathrm{N}(2)-\mathrm{C}(24)$ & $167.7(3)$ \\
\hline $\mathrm{S}(2)-\mathrm{N}(2)-\mathrm{C}(24)-\mathrm{C}(25)$ & $88.3(4)$ \\
\hline $\mathrm{S}(2)-\mathrm{N}(2)-\mathrm{C}(24)-\mathrm{C}(35)$ & $-148.4(3)$ \\
\hline $\mathrm{N}(2)-\mathrm{C}(24)-\mathrm{C}(25)-\mathrm{C}(26)$ & $-123.5(4)$ \\
\hline $\mathrm{C}(35)-\mathrm{C}(24)-\mathrm{C}(25)-\mathrm{C}(26)$ & $113.5(4)$ \\
\hline $\mathrm{N}(2)-\mathrm{C}(24)-\mathrm{C}(25)-\mathrm{C}(34)$ & $59.9(5)$ \\
\hline $\mathrm{C}(35)-\mathrm{C}(24)-\mathrm{C}(25)-\mathrm{C}(34)$ & $-63.1(5)$ \\
\hline$C(34)-C(25)-C(26)-C(27)$ & $2.6(6)$ \\
\hline$C(24)-C(25)-C(26)-C(27)$ & $-174.0(4)$ \\
\hline$C(25)-C(26)-C(27)-C(32)$ & $-0.8(6)$ \\
\hline
\end{tabular}




\begin{tabular}{|c|c|}
\hline $\mathrm{C}(25)-\mathrm{C}(26)-\mathrm{C}(27)-\mathrm{C}(28)$ & $176.9(4)$ \\
\hline $\mathrm{C}(32)-\mathrm{C}(27)-\mathrm{C}(28)-\mathrm{C}(29)$ & $1.5(7)$ \\
\hline $\mathrm{C}(26)-\mathrm{C}(27)-\mathrm{C}(28)-\mathrm{C}(29)$ & $-176.2(5)$ \\
\hline $\mathrm{C}(27)-\mathrm{C}(28)-\mathrm{C}(29)-\mathrm{C}(30)$ & $-1.1(9)$ \\
\hline$C(28)-C(29)-C(30)-C(31)$ & $-0.4(10)$ \\
\hline$C(29)-C(30)-C(31)-C(32)$ & $1.3(10)$ \\
\hline $\mathrm{C}(26)-\mathrm{C}(27)-\mathrm{C}(32)-\mathrm{C}(33)$ & $-1.6(7)$ \\
\hline $\mathrm{C}(28)-\mathrm{C}(27)-\mathrm{C}(32)-\mathrm{C}(33)$ & $-179.3(5)$ \\
\hline $\mathrm{C}(26)-\mathrm{C}(27)-\mathrm{C}(32)-\mathrm{C}(31)$ & 177.2(4) \\
\hline $\mathrm{C}(28)-\mathrm{C}(27)-\mathrm{C}(32)-\mathrm{C}(31)$ & $-0.5(7)$ \\
\hline $\mathrm{C}(30)-\mathrm{C}(31)-\mathrm{C}(32)-\mathrm{C}(27)$ & $-0.9(8)$ \\
\hline $\mathrm{C}(30)-\mathrm{C}(31)-\mathrm{C}(32)-\mathrm{C}(33)$ & $177.9(6)$ \\
\hline $\mathrm{C}(27)-\mathrm{C}(32)-\mathrm{C}(33)-\mathrm{C}(34)$ & $2.2(7)$ \\
\hline $\mathrm{C}(31)-\mathrm{C}(32)-\mathrm{C}(33)-\mathrm{C}(34)$ & $-176.5(5)$ \\
\hline $\mathrm{C}(32)-\mathrm{C}(33)-\mathrm{C}(34)-\mathrm{C}(25)$ & $-0.5(7)$ \\
\hline$C(26)-C(25)-C(34)-C(33)$ & $-1.9(7)$ \\
\hline $\mathrm{C}(24)-\mathrm{C}(25)-\mathrm{C}(34)-\mathrm{C}(33)$ & $174.6(4)$ \\
\hline $\mathrm{N}(2)-\mathrm{C}(24)-\mathrm{C}(35)-\mathrm{P}(2)$ & $78.4(4)$ \\
\hline $\mathrm{C}(25)-\mathrm{C}(24)-\mathrm{C}(35)-\mathrm{P}(2)$ & $-154.9(3)$ \\
\hline $\mathrm{O}(5)-\mathrm{P}(2)-\mathrm{C}(35)-\mathrm{C}(24)$ & $50.4(4)$ \\
\hline $\mathrm{O}(6)-\mathrm{P}(2)-\mathrm{C}(35)-\mathrm{C}(24)$ & $-76.2(4)$ \\
\hline $\mathrm{C}(36)-\mathrm{P}(2)-\mathrm{C}(35)-\mathrm{C}(24)$ & $172.6(3)$ \\
\hline $\mathrm{O}(5)-\mathrm{P}(2)-\mathrm{C}(36)-\mathrm{C}(41)$ & $-169.7(4)$ \\
\hline $\mathrm{O}(6)-\mathrm{P}(2)-\mathrm{C}(36)-\mathrm{C}(41)$ & $-43.4(4)$ \\
\hline $\mathrm{C}(35)-\mathrm{P}(2)-\mathrm{C}(36)-\mathrm{C}(41)$ & $66.0(4)$ \\
\hline $\mathrm{O}(5)-\mathrm{P}(2)-\mathrm{C}(36)-\mathrm{C}(37)$ & $13.8(4)$ \\
\hline $\mathrm{O}(6)-\mathrm{P}(2)-\mathrm{C}(36)-\mathrm{C}(37)$ & 140.1(4) \\
\hline $\mathrm{C}(35)-\mathrm{P}(2)-\mathrm{C}(36)-\mathrm{C}(37)$ & $-110.6(4)$ \\
\hline $\mathrm{C}(41)-\mathrm{C}(36)-\mathrm{C}(37)-\mathrm{C}(38)$ & $-0.1(7)$ \\
\hline $\mathrm{P}(2)-\mathrm{C}(36)-\mathrm{C}(37)-\mathrm{C}(38)$ & $176.5(4)$ \\
\hline $\mathrm{C}(36)-\mathrm{C}(37)-\mathrm{C}(38)-\mathrm{C}(39)$ & $-0.2(8)$ \\
\hline $\mathrm{C}(37)-\mathrm{C}(38)-\mathrm{C}(39)-\mathrm{C}(40)$ & $0.6(9)$ \\
\hline$C(38)-C(39)-C(40)-C(41)$ & $-0.5(9)$ \\
\hline$C(37)-C(36)-C(41)-C(40)$ & $0.2(7)$ \\
\hline $\mathrm{P}(2)-\mathrm{C}(36)-\mathrm{C}(41)-\mathrm{C}(40)$ & $-176.4(4)$ \\
\hline $\mathrm{C}(39)-\mathrm{C}(40)-\mathrm{C}(41)-\mathrm{C}(36)$ & $0.2(8)$ \\
\hline $\mathrm{O}(4)-\mathrm{S}(2)-\mathrm{C}(43)-\mathrm{C}(45)$ & $-48.4(4)$ \\
\hline $\mathrm{N}(2)-\mathrm{S}(2)-\mathrm{C}(43)-\mathrm{C}(45)$ & $68.4(3)$ \\
\hline
\end{tabular}


$\mathrm{O}(4)-\mathrm{S}(2)-\mathrm{C}(43)-\mathrm{C}(44)$

$\mathrm{N}(2)-\mathrm{S}(2)-\mathrm{C}(43)-\mathrm{C}(44)$

$\mathrm{O}(4)-\mathrm{S}(2)-\mathrm{C}(43)-\mathrm{C}(46)$

Symmetry transformations used to generate equivalent atoms:

Table 7. Hydrogen bonds for compound compound $10(R, S, S)\left[\AA\right.$ and $\left.^{\circ}\right]$.

\begin{tabular}{lllll}
\hline D-H...A & d(D-H) & $d(H . . . A)$ & $d(D . . A)$ & $<(\mathrm{DHA})$ \\
\hline $\mathrm{N}(2)-\mathrm{H}(2 \mathrm{~N}) \ldots \mathrm{O}(5) \# 1$ & $0.90(2)$ & $2.18(3)$ & $2.994(5)$ & $152(4)$ \\
$\mathrm{N}(1)-\mathrm{H}(1 \mathrm{~N}) \ldots \mathrm{O}(2) \# 1$ & $0.89(2)$ & $2.20(3)$ & $3.016(5)$ & $153(4)$
\end{tabular}

Symmetry transformations used to generate equivalent atoms:

$\# 1 \mathrm{x}, \mathrm{y}-1, \mathrm{z}$

Methyl 2-(tert-butylsulfinamide)-2-(isopropyl)ethyl phenylphosphinate, $12(R, S, R)$<smiles></smiles>

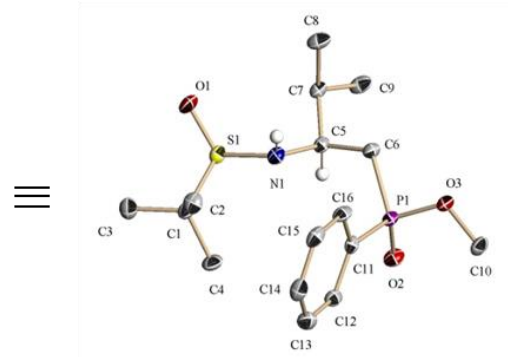

Table 1. Crystal data and structure refinement for compound $12(R, S, R)$.

Empirical formula

Formula weight

Temperature

Wavelength

Crystal system

Space group

Unit cell dimensions

Volume
$\mathrm{C}_{16} \mathrm{H}_{28} \mathrm{NO}_{3} \mathrm{PS}$

345.42

173(2) K

$0.71073 \AA$

Orthorhombic

P $22_{1} 2_{1} 2_{1}$

$\mathrm{a}=5.9396(3) \AA \quad \alpha=90^{\circ}$.

$\mathrm{b}=17.3738(10) \AA \quad \beta=90^{\circ}$.

$\mathrm{c}=17.4694(10) \AA \quad \gamma=90^{\circ}$.

1802.73(17) $\AA^{3}$ 
Z

Density (calculated)

Absorption coefficient

$\mathrm{F}(000)$

Crystal size

Theta range for data collection

Index ranges

Reflections collected

Independent reflections

Completeness to theta $=25.25^{\circ}$

Absorption correction

Max. and min. transmission

Refinement method

Data / restraints / parameters

Goodness-of-fit on $\mathrm{F}^{2}$

Final $\mathrm{R}$ indices [I $>2 \operatorname{sigma}(\mathrm{I})]$

$\mathrm{R}$ indices (all data)

Absolute structure parameter

Largest diff. peak and hole
4

$1.273 \mathrm{Mg} / \mathrm{m}^{3}$

$0.280 \mathrm{~mm}^{-1}$

744

$0.30 \times 0.25 \times 0.10 \mathrm{~mm}^{3}$

1.65 to $25.25^{\circ}$.

$-7<=\mathrm{h}<=7,-20<=\mathrm{k}<=20,-20<=\mathrm{l}<=20$

32350

$3257[\mathrm{R}(\mathrm{int})=0.0692]$

$100.0 \%$

Semi-empirical from equivalents

0.9726 and 0.9208

Full-matrix least-squares on $\mathrm{F}^{2}$

3257 / 1 / 208

1.127

$\mathrm{R} 1=0.0391, w \mathrm{R} 2=0.1080$

$\mathrm{R} 1=0.0456, \mathrm{wR} 2=0.1125$

$-0.03(10)$

0.629 and -0.530 e. $\AA^{-3}$

Table 2. Atomic coordinates $\left(\times 10^{4}\right)$ and equivalent isotropic displacement parameters $\left(\AA^{2} \times 10^{3}\right)$ for compound $\mathbf{1 2}(\boldsymbol{R}, S, \boldsymbol{R})$. U(eq) is defined as one third of the trace of the orthogonalized $\mathrm{U}^{\mathrm{ij}}$ tensor.

\begin{tabular}{|c|c|c|c|c|}
\hline & $\mathrm{x}$ & $\mathrm{y}$ & $\mathrm{z}$ & $\mathrm{U}(\mathrm{eq})$ \\
\hline$S(1)$ & $51(1)$ & $9426(1)$ & $8902(1)$ & $20(1)$ \\
\hline $\mathrm{P}(1)$ & $4170(1)$ & $8128(1)$ & $6914(1)$ & $16(1)$ \\
\hline $\mathrm{O}(1)$ & $-2348(4)$ & $9361(1)$ & $9158(1)$ & $30(1)$ \\
\hline $\mathrm{O}(2)$ & $6420(3)$ & $8313(1)$ & $7223(1)$ & $23(1)$ \\
\hline $\mathrm{O}(3)$ & $4150(3)$ & $7438(1)$ & 6311(1) & 21(1) \\
\hline $\mathrm{N}(1)$ & $513(4)$ & $8842(1)$ & $8158(1)$ & $19(1)$ \\
\hline $\mathrm{C}(1)$ & $275(5)$ & $10354(2)$ & $8403(2)$ & $20(1)$ \\
\hline$C(2)$ & $-1190(5)$ & $10361(2)$ & $7688(2)$ & $25(1)$ \\
\hline$C(3)$ & $-505(5)$ & $10955(2)$ & $8986(2)$ & $27(1)$ \\
\hline $\mathrm{C}(4)$ & $2760(5)$ & $10461(2)$ & $8208(2)$ & $27(1)$ \\
\hline$C(5)$ & $1863(4)$ & $8145(2)$ & $8333(1)$ & $17(1)$ \\
\hline
\end{tabular}




\begin{tabular}{lrrrr}
$\mathrm{C}(6)$ & $2205(5)$ & $7712(2)$ & $7580(2)$ & $19(1)$ \\
$\mathrm{C}(7)$ & $844(5)$ & $7638(2)$ & $8968(2)$ & $19(1)$ \\
$\mathrm{C}(8)$ & $-1453(5)$ & $7300(2)$ & $8762(2)$ & $27(1)$ \\
$\mathrm{C}(9)$ & $2463(5)$ & $7007(2)$ & $9217(2)$ & $25(1)$ \\
$\mathrm{C}(10)$ & $5628(5)$ & $7465(2)$ & $5655(2)$ & $25(1)$ \\
$\mathrm{C}(11)$ & $2977(4)$ & $8926(2)$ & $6400(1)$ & $17(1)$ \\
$\mathrm{C}(12)$ & $4258(5)$ & $9589(2)$ & $6288(2)$ & $20(1)$ \\
$\mathrm{C}(13)$ & $3442(5)$ & $10189(2)$ & $5841(2)$ & $26(1)$ \\
$\mathrm{C}(14)$ & $1321(5)$ & $10129(2)$ & $5508(2)$ & $25(1)$ \\
$\mathrm{C}(15)$ & $25(5)$ & $9484(2)$ & $5628(2)$ & $24(1)$ \\
$\mathrm{C}(16)$ & $827(5)$ & $8879(2)$ & $6071(2)$ & $22(1)$ \\
\hline
\end{tabular}

Table 3. Bond lengths $[\AA]$ and angles $\left[{ }^{\circ}\right]$ for compound $\mathbf{1 2}(\boldsymbol{R}, S, \boldsymbol{R})$.

\begin{tabular}{ll}
\hline $\mathrm{S}(1)-\mathrm{O}(1)$ & $1.498(2)$ \\
$\mathrm{S}(1)-\mathrm{N}(1)$ & $1.671(2)$ \\
$\mathrm{S}(1)-\mathrm{C}(1)$ & $1.838(3)$ \\
$\mathrm{P}(1)-\mathrm{O}(2)$ & $1.477(2)$ \\
$\mathrm{P}(1)-\mathrm{O}(3)$ & $1.595(2)$ \\
$\mathrm{P}(1)-\mathrm{C}(11)$ & $1.797(3)$ \\
$\mathrm{P}(1)-\mathrm{C}(6)$ & $1.800(3)$ \\
$\mathrm{O}(3)-\mathrm{C}(10)$ & $1.444(3)$ \\
$\mathrm{N}(1)-\mathrm{C}(5)$ & $1.485(3)$ \\
$\mathrm{N}(1)-\mathrm{H}(1 \mathrm{~N})$ & $0.824(18)$ \\
$\mathrm{C}(1)-\mathrm{C}(2)$ & $1.522(4)$ \\
$\mathrm{C}(1)-\mathrm{C}(4)$ & $1.526(4)$ \\
$\mathrm{C}(1)-\mathrm{C}(3)$ & $1.531(4)$ \\
$\mathrm{C}(2)-\mathrm{H}(2 \mathrm{~A})$ & 0.9800 \\
$\mathrm{C}(2)-\mathrm{H}(2 \mathrm{~B})$ & 0.9800 \\
$\mathrm{C}(2)-\mathrm{H}(2 \mathrm{C})$ & 0.9800 \\
$\mathrm{C}(3)-\mathrm{H}(3 \mathrm{~A})$ & 0.9800 \\
$\mathrm{C}(3)-\mathrm{H}(3 \mathrm{~B})$ & 0.9800 \\
$\mathrm{C}(3)-\mathrm{H}(3 \mathrm{C})$ & 0.9800 \\
$\mathrm{C}(4)-\mathrm{H}(4 \mathrm{~A})$ & 0.9800 \\
$\mathrm{C}(4)-\mathrm{H}(4 \mathrm{~B})$ & 0.9800 \\
$\mathrm{C}(4)-\mathrm{H}(4 \mathrm{C})$ & 0.9800 \\
$\mathrm{C}(5)-\mathrm{C}(6)$ & $1.530(4)$ \\
& \\
&
\end{tabular}




\begin{tabular}{|c|c|}
\hline$C(5)-C(7)$ & $1.541(4)$ \\
\hline $\mathrm{C}(5)-\mathrm{H}(5)$ & 1.0000 \\
\hline $\mathrm{C}(6)-\mathrm{H}(6 \mathrm{~A})$ & 0.9900 \\
\hline $\mathrm{C}(6)-\mathrm{H}(6 \mathrm{~B})$ & 0.9900 \\
\hline$C(7)-C(9)$ & $1.522(4)$ \\
\hline$C(7)-C(8)$ & $1.528(4)$ \\
\hline $\mathrm{C}(7)-\mathrm{H}(7)$ & 1.0000 \\
\hline $\mathrm{C}(8)-\mathrm{H}(8 \mathrm{~A})$ & 0.9800 \\
\hline $\mathrm{C}(8)-\mathrm{H}(8 \mathrm{~B})$ & 0.9800 \\
\hline $\mathrm{C}(8)-\mathrm{H}(8 \mathrm{C})$ & 0.9800 \\
\hline $\mathrm{C}(9)-\mathrm{H}(9 \mathrm{~A})$ & 0.9800 \\
\hline $\mathrm{C}(9)-\mathrm{H}(9 \mathrm{~B})$ & 0.9800 \\
\hline $\mathrm{C}(9)-\mathrm{H}(9 \mathrm{C})$ & 0.9800 \\
\hline $\mathrm{C}(10)-\mathrm{H}(10 \mathrm{~A})$ & 0.9800 \\
\hline $\mathrm{C}(10)-\mathrm{H}(10 \mathrm{~B})$ & 0.9800 \\
\hline $\mathrm{C}(10)-\mathrm{H}(10 \mathrm{C})$ & 0.9800 \\
\hline $\mathrm{C}(11)-\mathrm{C}(12)$ & $1.394(4)$ \\
\hline$C(11)-C(16)$ & $1.403(4)$ \\
\hline $\mathrm{C}(12)-\mathrm{C}(13)$ & $1.390(4)$ \\
\hline $\mathrm{C}(12)-\mathrm{H}(12)$ & 0.9500 \\
\hline$C(13)-C(14)$ & $1.392(4)$ \\
\hline $\mathrm{C}(13)-\mathrm{H}(13)$ & 0.9500 \\
\hline$C(14)-C(15)$ & $1.376(4)$ \\
\hline $\mathrm{C}(14)-\mathrm{H}(14)$ & 0.9500 \\
\hline$C(15)-C(16)$ & $1.390(4)$ \\
\hline $\mathrm{C}(15)-\mathrm{H}(15)$ & 0.9500 \\
\hline $\mathrm{C}(16)-\mathrm{H}(16)$ & 0.9500 \\
\hline $\mathrm{O}(1)-\mathrm{S}(1)-\mathrm{N}(1)$ & $110.01(12)$ \\
\hline $\mathrm{O}(1)-\mathrm{S}(1)-\mathrm{C}(1)$ & $106.04(12)$ \\
\hline $\mathrm{N}(1)-\mathrm{S}(1)-\mathrm{C}(1)$ & $98.70(12)$ \\
\hline $\mathrm{O}(2)-\mathrm{P}(1)-\mathrm{O}(3)$ & $114.37(11)$ \\
\hline $\mathrm{O}(2)-\mathrm{P}(1)-\mathrm{C}(11)$ & $111.76(12)$ \\
\hline $\mathrm{O}(3)-\mathrm{P}(1)-\mathrm{C}(11)$ & $104.33(11)$ \\
\hline $\mathrm{O}(2)-\mathrm{P}(1)-\mathrm{C}(6)$ & $115.93(12)$ \\
\hline $\mathrm{O}(3)-\mathrm{P}(1)-\mathrm{C}(6)$ & $96.93(12)$ \\
\hline $\mathrm{C}(11)-\mathrm{P}(1)-\mathrm{C}(6)$ & $112.14(13)$ \\
\hline $\mathrm{C}(10)-\mathrm{O}(3)-\mathrm{P}(1)$ & $119.66(17)$ \\
\hline
\end{tabular}




\begin{tabular}{|c|c|}
\hline $\mathrm{C}(5)-\mathrm{N}(1)-\mathrm{S}(1)$ & $115.04(17)$ \\
\hline $\mathrm{C}(5)-\mathrm{N}(1)-\mathrm{H}(1 \mathrm{~N})$ & 112(2) \\
\hline $\mathrm{S}(1)-\mathrm{N}(1)-\mathrm{H}(1 \mathrm{~N})$ & $112(2)$ \\
\hline$C(2)-C(1)-C(4)$ & $111.7(2)$ \\
\hline$C(2)-C(1)-C(3)$ & $111.6(2)$ \\
\hline $\mathrm{C}(4)-\mathrm{C}(1)-\mathrm{C}(3)$ & $111.0(2)$ \\
\hline $\mathrm{C}(2)-\mathrm{C}(1)-\mathrm{S}(1)$ & $110.77(19)$ \\
\hline $\mathrm{C}(4)-\mathrm{C}(1)-\mathrm{S}(1)$ & $106.40(19)$ \\
\hline$C(3)-C(1)-S(1)$ & 105.07(19) \\
\hline $\mathrm{C}(1)-\mathrm{C}(2)-\mathrm{H}(2 \mathrm{~A})$ & 109.5 \\
\hline $\mathrm{C}(1)-\mathrm{C}(2)-\mathrm{H}(2 \mathrm{~B})$ & 109.5 \\
\hline $\mathrm{H}(2 \mathrm{~A})-\mathrm{C}(2)-\mathrm{H}(2 \mathrm{~B})$ & 109.5 \\
\hline $\mathrm{C}(1)-\mathrm{C}(2)-\mathrm{H}(2 \mathrm{C})$ & 109.5 \\
\hline $\mathrm{H}(2 \mathrm{~A})-\mathrm{C}(2)-\mathrm{H}(2 \mathrm{C})$ & 109.5 \\
\hline $\mathrm{H}(2 \mathrm{~B})-\mathrm{C}(2)-\mathrm{H}(2 \mathrm{C})$ & 109.5 \\
\hline $\mathrm{C}(1)-\mathrm{C}(3)-\mathrm{H}(3 \mathrm{~A})$ & 109.5 \\
\hline $\mathrm{C}(1)-\mathrm{C}(3)-\mathrm{H}(3 \mathrm{~B})$ & 109.5 \\
\hline $\mathrm{H}(3 \mathrm{~A})-\mathrm{C}(3)-\mathrm{H}(3 \mathrm{~B})$ & 109.5 \\
\hline $\mathrm{C}(1)-\mathrm{C}(3)-\mathrm{H}(3 \mathrm{C})$ & 109.5 \\
\hline $\mathrm{H}(3 \mathrm{~A})-\mathrm{C}(3)-\mathrm{H}(3 \mathrm{C})$ & 109.5 \\
\hline $\mathrm{H}(3 \mathrm{~B})-\mathrm{C}(3)-\mathrm{H}(3 \mathrm{C})$ & 109.5 \\
\hline $\mathrm{C}(1)-\mathrm{C}(4)-\mathrm{H}(4 \mathrm{~A})$ & 109.5 \\
\hline $\mathrm{C}(1)-\mathrm{C}(4)-\mathrm{H}(4 \mathrm{~B})$ & 109.5 \\
\hline $\mathrm{H}(4 \mathrm{~A})-\mathrm{C}(4)-\mathrm{H}(4 \mathrm{~B})$ & 109.5 \\
\hline $\mathrm{C}(1)-\mathrm{C}(4)-\mathrm{H}(4 \mathrm{C})$ & 109.5 \\
\hline $\mathrm{H}(4 \mathrm{~A})-\mathrm{C}(4)-\mathrm{H}(4 \mathrm{C})$ & 109.5 \\
\hline $\mathrm{H}(4 \mathrm{~B})-\mathrm{C}(4)-\mathrm{H}(4 \mathrm{C})$ & 109.5 \\
\hline$N(1)-C(5)-C(6)$ & $107.2(2)$ \\
\hline$N(1)-C(5)-C(7)$ & $113.8(2)$ \\
\hline$C(6)-C(5)-C(7)$ & $112.9(2)$ \\
\hline $\mathrm{N}(1)-\mathrm{C}(5)-\mathrm{H}(5)$ & 107.6 \\
\hline $\mathrm{C}(6)-\mathrm{C}(5)-\mathrm{H}(5)$ & 107.6 \\
\hline $\mathrm{C}(7)-\mathrm{C}(5)-\mathrm{H}(5)$ & 107.6 \\
\hline$C(5)-C(6)-P(1)$ & $116.41(19)$ \\
\hline $\mathrm{C}(5)-\mathrm{C}(6)-\mathrm{H}(6 \mathrm{~A})$ & 108.2 \\
\hline$P(1)-C(6)-H(6 A)$ & 108.2 \\
\hline $\mathrm{C}(5)-\mathrm{C}(6)-\mathrm{H}(6 \mathrm{~B})$ & 108.2 \\
\hline$P(1)-C(6)-H(6 B)$ & 108.2 \\
\hline
\end{tabular}




\begin{tabular}{|c|c|}
\hline $\mathrm{H}(6 \mathrm{~A})-\mathrm{C}(6)-\mathrm{H}(6 \mathrm{~B})$ & 107.3 \\
\hline$C(9)-C(7)-C(8)$ & $110.8(2)$ \\
\hline$C(9)-C(7)-C(5)$ & $111.7(2)$ \\
\hline$C(8)-C(7)-C(5)$ & $113.7(2)$ \\
\hline $\mathrm{C}(9)-\mathrm{C}(7)-\mathrm{H}(7)$ & 106.8 \\
\hline $\mathrm{C}(8)-\mathrm{C}(7)-\mathrm{H}(7)$ & 106.8 \\
\hline $\mathrm{C}(5)-\mathrm{C}(7)-\mathrm{H}(7)$ & 106.8 \\
\hline $\mathrm{C}(7)-\mathrm{C}(8)-\mathrm{H}(8 \mathrm{~A})$ & 109.5 \\
\hline $\mathrm{C}(7)-\mathrm{C}(8)-\mathrm{H}(8 \mathrm{~B})$ & 109.5 \\
\hline $\mathrm{H}(8 \mathrm{~A})-\mathrm{C}(8)-\mathrm{H}(8 \mathrm{~B})$ & 109.5 \\
\hline $\mathrm{C}(7)-\mathrm{C}(8)-\mathrm{H}(8 \mathrm{C})$ & 109.5 \\
\hline $\mathrm{H}(8 \mathrm{~A})-\mathrm{C}(8)-\mathrm{H}(8 \mathrm{C})$ & 109.5 \\
\hline $\mathrm{H}(8 \mathrm{~B})-\mathrm{C}(8)-\mathrm{H}(8 \mathrm{C})$ & 109.5 \\
\hline $\mathrm{C}(7)-\mathrm{C}(9)-\mathrm{H}(9 \mathrm{~A})$ & 109.5 \\
\hline $\mathrm{C}(7)-\mathrm{C}(9)-\mathrm{H}(9 \mathrm{~B})$ & 109.5 \\
\hline $\mathrm{H}(9 \mathrm{~A})-\mathrm{C}(9)-\mathrm{H}(9 \mathrm{~B})$ & 109.5 \\
\hline $\mathrm{C}(7)-\mathrm{C}(9)-\mathrm{H}(9 \mathrm{C})$ & 109.5 \\
\hline $\mathrm{H}(9 \mathrm{~A})-\mathrm{C}(9)-\mathrm{H}(9 \mathrm{C})$ & 109.5 \\
\hline $\mathrm{H}(9 \mathrm{~B})-\mathrm{C}(9)-\mathrm{H}(9 \mathrm{C})$ & 109.5 \\
\hline $\mathrm{O}(3)-\mathrm{C}(10)-\mathrm{H}(10 \mathrm{~A})$ & 109.5 \\
\hline $\mathrm{O}(3)-\mathrm{C}(10)-\mathrm{H}(10 \mathrm{~B})$ & 109.5 \\
\hline $\mathrm{H}(10 \mathrm{~A})-\mathrm{C}(10)-\mathrm{H}(10 \mathrm{~B})$ & 109.5 \\
\hline $\mathrm{O}(3)-\mathrm{C}(10)-\mathrm{H}(10 \mathrm{C})$ & 109.5 \\
\hline $\mathrm{H}(10 \mathrm{~A})-\mathrm{C}(10)-\mathrm{H}(10 \mathrm{C})$ & 109.5 \\
\hline$H(10 B)-C(10)-H(10 C)$ & 109.5 \\
\hline$C(12)-C(11)-C(16)$ & $119.2(3)$ \\
\hline $\mathrm{C}(12)-\mathrm{C}(11)-\mathrm{P}(1)$ & $119.5(2)$ \\
\hline $\mathrm{C}(16)-\mathrm{C}(11)-\mathrm{P}(1)$ & $121.2(2)$ \\
\hline$C(13)-C(12)-C(11)$ & $120.6(3)$ \\
\hline $\mathrm{C}(13)-\mathrm{C}(12)-\mathrm{H}(12)$ & 119.7 \\
\hline $\mathrm{C}(11)-\mathrm{C}(12)-\mathrm{H}(12)$ & 119.7 \\
\hline$C(12)-C(13)-C(14)$ & $119.6(3)$ \\
\hline $\mathrm{C}(12)-\mathrm{C}(13)-\mathrm{H}(13)$ & 120.2 \\
\hline $\mathrm{C}(14)-\mathrm{C}(13)-\mathrm{H}(13)$ & 120.2 \\
\hline$C(15)-C(14)-C(13)$ & $120.2(3)$ \\
\hline $\mathrm{C}(15)-\mathrm{C}(14)-\mathrm{H}(14)$ & 119.9 \\
\hline $\mathrm{C}(13)-\mathrm{C}(14)-\mathrm{H}(14)$ & 119.9 \\
\hline$C(14)-C(15)-C(16)$ & $120.6(3)$ \\
\hline
\end{tabular}




$\begin{array}{ll}\mathrm{C}(14)-\mathrm{C}(15)-\mathrm{H}(15) & 119.7 \\ \mathrm{C}(16)-\mathrm{C}(15)-\mathrm{H}(15) & 119.7 \\ \mathrm{C}(15)-\mathrm{C}(16)-\mathrm{C}(11) & 119.7(3) \\ \mathrm{C}(15)-\mathrm{C}(16)-\mathrm{H}(16) & 120.1 \\ \mathrm{C}(11)-\mathrm{C}(16)-\mathrm{H}(16) & 120.1\end{array}$

Symmetry transformations used to generate equivalent atoms:

Table 4. Anisotropic displacement parameters $\left(\AA^{2} \times 10^{3}\right)$ for compound $\mathbf{1 2}(\boldsymbol{R}, \boldsymbol{S}, \boldsymbol{R})$.

The anisotropic displacement factor exponent takes the form: $-2 \pi^{2}\left[h^{2} a^{* 2} U^{11}+\ldots+2 h k\right.$ $a^{*} b^{*} U^{12}$ ]

\begin{tabular}{lcccccc}
\hline & $\mathrm{U}^{11}$ & $\mathrm{U}^{22}$ & $\mathrm{U}^{33}$ & $\mathrm{U}^{23}$ & $\mathrm{U}^{13}$ & $\mathrm{U}^{12}$ \\
\hline $\mathrm{S}(1)$ & $20(1)$ & $24(1)$ & $17(1)$ & $-1(1)$ & $1(1)$ & $2(1)$ \\
$\mathrm{P}(1)$ & $12(1)$ & $21(1)$ & $16(1)$ & $0(1)$ & $1(1)$ & $-1(1)$ \\
$\mathrm{O}(1)$ & $26(1)$ & $33(1)$ & $31(1)$ & $1(1)$ & $16(1)$ & $1(1)$ \\
$\mathrm{O}(2)$ & $14(1)$ & $32(1)$ & $23(1)$ & $2(1)$ & $-4(1)$ & $-1(1)$ \\
$\mathrm{O}(3)$ & $16(1)$ & $24(1)$ & $24(1)$ & $-1(1)$ & $6(1)$ & $-1(1)$ \\
$\mathrm{N}(1)$ & $14(1)$ & $24(1)$ & $19(1)$ & $1(1)$ & $-3(1)$ & $1(1)$ \\
$\mathrm{C}(1)$ & $14(1)$ & $24(1)$ & $21(1)$ & $-2(1)$ & $0(1)$ & $0(1)$ \\
$\mathrm{C}(2)$ & $20(2)$ & $29(2)$ & $25(2)$ & $2(1)$ & $-6(1)$ & $5(1)$ \\
$\mathrm{C}(3)$ & $30(2)$ & $25(2)$ & $26(2)$ & $-2(1)$ & $1(1)$ & $3(1)$ \\
$\mathrm{C}(4)$ & $16(2)$ & $33(2)$ & $32(2)$ & $-4(1)$ & $4(1)$ & $-7(1)$ \\
$\mathrm{C}(5)$ & $14(1)$ & $21(1)$ & $16(1)$ & $0(1)$ & $-1(1)$ & $1(1)$ \\
$\mathrm{C}(6)$ & $16(1)$ & $22(1)$ & $18(1)$ & $1(1)$ & $2(1)$ & $-2(1)$ \\
$\mathrm{C}(7)$ & $15(1)$ & $24(1)$ & $17(1)$ & $1(1)$ & $1(1)$ & $1(1)$ \\
$\mathrm{C}(8)$ & $17(1)$ & $36(2)$ & $28(2)$ & $10(1)$ & $0(1)$ & $-2(1)$ \\
$\mathrm{C}(9)$ & $15(1)$ & $35(2)$ & $26(2)$ & $11(1)$ & $0(1)$ & $3(1)$ \\
$\mathrm{C}(10)$ & $25(2)$ & $30(2)$ & $20(1)$ & $-3(1)$ & $9(1)$ & $0(1)$ \\
$\mathrm{C}(11)$ & $18(1)$ & $21(1)$ & $12(1)$ & $-4(1)$ & $0(1)$ & $3(1)$ \\
$\mathrm{C}(12)$ & $17(1)$ & $25(1)$ & $18(1)$ & $-2(1)$ & $0(1)$ & $-3(1)$ \\
$\mathrm{C}(13)$ & $26(2)$ & $26(2)$ & $26(2)$ & $3(1)$ & $3(1)$ & $-2(1)$ \\
$\mathrm{C}(14)$ & $28(2)$ & $29(2)$ & $19(2)$ & $4(1)$ & $1(1)$ & $10(1)$ \\
$\mathrm{C}(15)$ & $16(1)$ & $39(2)$ & $17(1)$ & $-4(1)$ & $-1(1)$ & $3(1)$ \\
$\mathrm{C}(16)$ & $13(1)$ & $31(2)$ & $21(1)$ & $-2(1)$ & $1(1)$ & $-4(1)$ \\
\hline & & & & & \\
\hline
\end{tabular}


Table 5. Hydrogen coordinates $\left(\mathrm{x} 10^{4}\right)$ and isotropic displacement parameters $\left(\AA^{2} \times 10^{3}\right)$ for compound $12(R, S, R)$.

\begin{tabular}{|c|c|c|c|c|}
\hline & $\mathrm{x}$ & $\mathrm{y}$ & $\mathrm{z}$ & $\mathrm{U}(\mathrm{eq})$ \\
\hline $\mathrm{H}(1 \mathrm{~N})$ & $-660(40)$ & $8740(18)$ & $7930(16)$ & 23 \\
\hline $\mathrm{H}(2 \mathrm{~A})$ & -1179 & 10877 & 7462 & 37 \\
\hline $\mathrm{H}(2 \mathrm{~B})$ & -2736 & 10220 & 7825 & 37 \\
\hline $\mathrm{H}(2 \mathrm{C})$ & -599 & 9989 & 7316 & 37 \\
\hline $\mathrm{H}(3 \mathrm{~A})$ & -322 & 11471 & 8769 & 41 \\
\hline $\mathrm{H}(3 \mathrm{~B})$ & 401 & 10910 & 9453 & 41 \\
\hline $\mathrm{H}(3 \mathrm{C})$ & -2094 & 10868 & 9110 & 41 \\
\hline $\mathrm{H}(4 \mathrm{~A})$ & 3221 & 10069 & 7836 & 40 \\
\hline $\mathrm{H}(4 \mathrm{~B})$ & 3664 & 10408 & 8675 & 40 \\
\hline $\mathrm{H}(4 \mathrm{C})$ & 2992 & 10974 & 7989 & 40 \\
\hline $\mathrm{H}(5)$ & 3377 & 8321 & 8513 & 20 \\
\hline $\mathrm{H}(6 \mathrm{~A})$ & 726 & 7667 & 7322 & 22 \\
\hline $\mathrm{H}(6 \mathrm{~B})$ & 2717 & 7183 & 7702 & 22 \\
\hline $\mathrm{H}(7)$ & 607 & 7978 & 9423 & 22 \\
\hline $\mathrm{H}(8 \mathrm{~A})$ & -1292 & 6953 & 8323 & 40 \\
\hline $\mathrm{H}(8 \mathrm{~B})$ & -2493 & 7717 & 8631 & 40 \\
\hline $\mathrm{H}(8 \mathrm{C})$ & -2047 & 7012 & 9201 & 40 \\
\hline $\mathrm{H}(9 \mathrm{~A})$ & 1945 & 6783 & 9701 & 38 \\
\hline $\mathrm{H}(9 \mathrm{~B})$ & 3970 & 7225 & 9286 & 38 \\
\hline $\mathrm{H}(9 \mathrm{C})$ & 2517 & 6605 & 8823 & 38 \\
\hline $\mathrm{H}(10 \mathrm{~A})$ & 5293 & 7925 & 5351 & 38 \\
\hline $\mathrm{H}(10 \mathrm{~B})$ & 5398 & 7004 & 5341 & 38 \\
\hline $\mathrm{H}(10 \mathrm{C})$ & 7196 & 7484 & 5829 & 38 \\
\hline $\mathrm{H}(12)$ & 5702 & 9630 & 6518 & 24 \\
\hline $\mathrm{H}(13)$ & 4327 & 10638 & 5765 & 31 \\
\hline $\mathrm{H}(14)$ & 767 & 10535 & 5196 & 30 \\
\hline $\mathrm{H}(15)$ & -1433 & 9452 & 5406 & 29 \\
\hline $\mathrm{H}(16)$ & -77 & 8434 & 6149 & 26 \\
\hline
\end{tabular}


Table 6. Torsion angles $\left[{ }^{\circ}\right]$ for $\mathbf{1 2}(\boldsymbol{R}, S, \boldsymbol{R})$.

\begin{tabular}{|c|c|}
\hline $\mathrm{O}(2)-\mathrm{P}(1)-\mathrm{O}(3)-\mathrm{C}(10)$ & $-53.5(2)$ \\
\hline $\mathrm{C}(11)-\mathrm{P}(1)-\mathrm{O}(3)-\mathrm{C}(10)$ & $68.9(2)$ \\
\hline $\mathrm{C}(6)-\mathrm{P}(1)-\mathrm{O}(3)-\mathrm{C}(10)$ & $-176.1(2)$ \\
\hline $\mathrm{O}(1)-\mathrm{S}(1)-\mathrm{N}(1)-\mathrm{C}(5)$ & $-106.85(19)$ \\
\hline $\mathrm{C}(1)-\mathrm{S}(1)-\mathrm{N}(1)-\mathrm{C}(5)$ & $142.47(18)$ \\
\hline $\mathrm{O}(1)-\mathrm{S}(1)-\mathrm{C}(1)-\mathrm{C}(2)$ & $-63.8(2)$ \\
\hline$N(1)-S(1)-C(1)-C(2)$ & $50.1(2)$ \\
\hline $\mathrm{O}(1)-\mathrm{S}(1)-\mathrm{C}(1)-\mathrm{C}(4)$ & $174.65(19)$ \\
\hline $\mathrm{N}(1)-\mathrm{S}(1)-\mathrm{C}(1)-\mathrm{C}(4)$ & $-71.5(2)$ \\
\hline $\mathrm{O}(1)-\mathrm{S}(1)-\mathrm{C}(1)-\mathrm{C}(3)$ & $56.9(2)$ \\
\hline $\mathrm{N}(1)-\mathrm{S}(1)-\mathrm{C}(1)-\mathrm{C}(3)$ & 170.71(19) \\
\hline$S(1)-N(1)-C(5)-C(6)$ & $-177.07(17)$ \\
\hline $\mathrm{S}(1)-\mathrm{N}(1)-\mathrm{C}(5)-\mathrm{C}(7)$ & $57.3(3)$ \\
\hline $\mathrm{N}(1)-\mathrm{C}(5)-\mathrm{C}(6)-\mathrm{P}(1)$ & $73.6(2)$ \\
\hline $\mathrm{C}(7)-\mathrm{C}(5)-\mathrm{C}(6)-\mathrm{P}(1)$ & $-160.35(19)$ \\
\hline $\mathrm{O}(2)-\mathrm{P}(1)-\mathrm{C}(6)-\mathrm{C}(5)$ & $51.9(2)$ \\
\hline $\mathrm{O}(3)-\mathrm{P}(1)-\mathrm{C}(6)-\mathrm{C}(5)$ & $173.3(2)$ \\
\hline $\mathrm{C}(11)-\mathrm{P}(1)-\mathrm{C}(6)-\mathrm{C}(5)$ & $-78.1(2)$ \\
\hline $\mathrm{N}(1)-\mathrm{C}(5)-\mathrm{C}(7)-\mathrm{C}(9)$ & $-170.7(2)$ \\
\hline $\mathrm{C}(6)-\mathrm{C}(5)-\mathrm{C}(7)-\mathrm{C}(9)$ & $66.8(3)$ \\
\hline $\mathrm{N}(1)-\mathrm{C}(5)-\mathrm{C}(7)-\mathrm{C}(8)$ & $63.1(3)$ \\
\hline $\mathrm{C}(6)-\mathrm{C}(5)-\mathrm{C}(7)-\mathrm{C}(8)$ & $-59.4(3)$ \\
\hline $\mathrm{O}(2)-\mathrm{P}(1)-\mathrm{C}(11)-\mathrm{C}(12)$ & $5.1(3)$ \\
\hline $\mathrm{O}(3)-\mathrm{P}(1)-\mathrm{C}(11)-\mathrm{C}(12)$ & $-119.0(2)$ \\
\hline $\mathrm{C}(6)-\mathrm{P}(1)-\mathrm{C}(11)-\mathrm{C}(12)$ & $137.2(2)$ \\
\hline $\mathrm{O}(2)-\mathrm{P}(1)-\mathrm{C}(11)-\mathrm{C}(16)$ & $-178.8(2)$ \\
\hline $\mathrm{O}(3)-\mathrm{P}(1)-\mathrm{C}(11)-\mathrm{C}(16)$ & $57.1(2)$ \\
\hline$C(6)-P(1)-C(11)-C(16)$ & $-46.7(2)$ \\
\hline $\mathrm{C}(16)-\mathrm{C}(11)-\mathrm{C}(12)-\mathrm{C}(13)$ & $-1.4(4)$ \\
\hline $\mathrm{P}(1)-\mathrm{C}(11)-\mathrm{C}(12)-\mathrm{C}(13)$ & $174.9(2)$ \\
\hline $\mathrm{C}(11)-\mathrm{C}(12)-\mathrm{C}(13)-\mathrm{C}(14)$ & $0.3(4)$ \\
\hline $\mathrm{C}(12)-\mathrm{C}(13)-\mathrm{C}(14)-\mathrm{C}(15)$ & $0.9(4)$ \\
\hline $\mathrm{C}(13)-\mathrm{C}(14)-\mathrm{C}(15)-\mathrm{C}(16)$ & $-1.2(4)$ \\
\hline $\mathrm{C}(14)-\mathrm{C}(15)-\mathrm{C}(16)-\mathrm{C}(11)$ & $0.2(4)$ \\
\hline$C(12)-C(11)-C(16)-C(15)$ & $1.1(4)$ \\
\hline
\end{tabular}


Symmetry transformations used to generate equivalent atoms:

Table 7. Hydrogen bonds for compound $12(\boldsymbol{R}, S, \boldsymbol{R})\left[\AA\right.$ and $\left.^{\circ}\right]$.

\begin{tabular}{lllll}
\hline $\mathrm{D}-\mathrm{H} . . . \mathrm{A}$ & $\mathrm{d}(\mathrm{D}-\mathrm{H})$ & $\mathrm{d}(\mathrm{H} \ldots \mathrm{A})$ & $\mathrm{d}(\mathrm{D} \ldots \mathrm{A})$ & $<(\mathrm{DHA})$ \\
\hline $\mathrm{N}(1)-\mathrm{H}(1 \mathrm{~N}) \ldots \mathrm{O}(2) \# 1$ & $0.824(18)$ & $2.253(19)$ & $3.069(3)$ & $171(3)$
\end{tabular}

Symmetry transformations used to generate equivalent atoms:

\#1 x-1,y,z

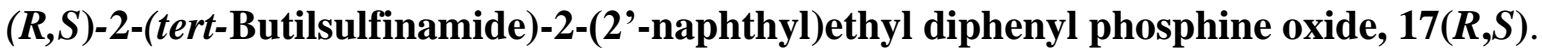

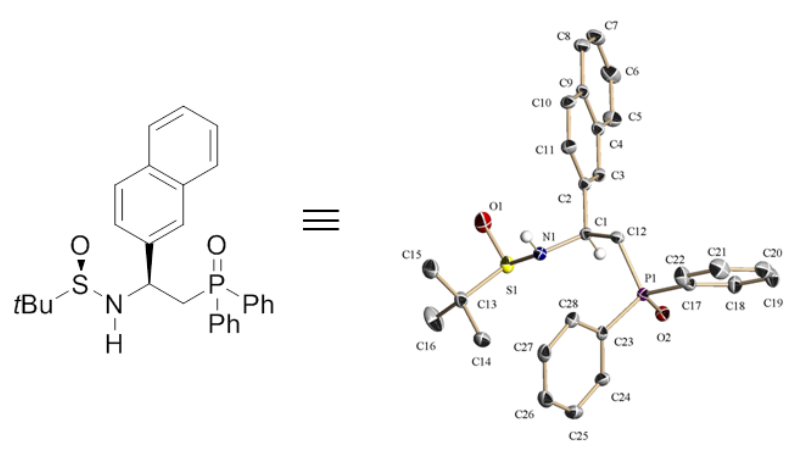

Table 1. Crystal data and structure refinement for compound $\mathbf{1 7}(\boldsymbol{R}, S)$.

Empirical formula

Formula weight

Temperature

Wavelength

Crystal system

Space group

Unit cell dimensions

Volume

$\mathrm{Z}$

Density (calculated)
$\mathrm{C}_{28} \mathrm{H}_{30} \mathrm{NO}_{2} \mathrm{PS}$

475.56

213(2) K

$0.71073 \AA$

Orthorhombic

P $22_{1} 2_{1}$

$\mathrm{a}=5.8765(6) \AA \quad \alpha=90^{\circ}$.

$\mathrm{b}=17.9914(16) \AA \quad \beta=90^{\circ}$.

$\mathrm{c}=23.770(2) \AA \quad \gamma=90^{\circ}$.

2513.1(4) $\AA^{3}$

4

$1.257 \mathrm{Mg} / \mathrm{m}^{3}$ 
Absorption coefficient

$\mathrm{F}(000)$

Crystal size

Theta range for data collection

Index ranges

Reflections collected

Independent reflections

Completeness to theta $=25.25^{\circ}$

Absorption correction

Max. and min. transmission

Refinement method

Data / restraints / parameters

Goodness-of-fit on $\mathrm{F}^{2}$

Final $\mathrm{R}$ indices $[\mathrm{I}>2 \operatorname{sigma}(\mathrm{I})]$

$\mathrm{R}$ indices (all data)

Absolute structure parameter

Largest diff. peak and hole
$0.218 \mathrm{~mm}^{-1}$

1008

$0.50 \times 0.30 \times 0.10 \mathrm{~mm}^{3}$

1.42 to $25.25^{\circ}$.

$-7<=\mathrm{h}<=6,-21<=\mathrm{k}<=15,-23<=\mathrm{l}<=21$

17551

$4453[\mathrm{R}(\mathrm{int})=0.0326]$

$99.8 \%$

Semi-empirical from equivalents

0.9786 and 0.9260

Full-matrix least-squares on $\mathrm{F}^{2}$

4453 / 1 / 304

1.021

$\mathrm{R} 1=0.0352, \mathrm{wR} 2=0.0858$

$\mathrm{R} 1=0.0395, \mathrm{wR} 2=0.0877$

$0.03(8)$

0.391 and -0.205 e. $\AA^{-3}$

Table 2. Atomic coordinates $\left(\times 10^{4}\right)$ and equivalent isotropic displacement parameters $\left(\AA^{2} \times 10^{3}\right)$ for compound $\mathbf{1 7}(\boldsymbol{R}, S) . \mathrm{U}(\mathrm{eq})$ is defined as one third of the trace of the orthogonalized $\mathrm{U}^{\mathrm{ij}}$ tensor.

\begin{tabular}{lcccc}
\hline & $\mathrm{x}$ & $\mathrm{y}$ & $\mathrm{z}$ & $\mathrm{U}(\mathrm{eq})$ \\
\hline $\mathrm{S}(1)$ & $2247(1)$ & $4085(1)$ & $2442(1)$ & $32(1)$ \\
$\mathrm{P}(1)$ & $5928(1)$ & $5572(1)$ & $1092(1)$ & $23(1)$ \\
$\mathrm{O}(1)$ & $687(4)$ & $3442(1)$ & $2423(1)$ & $53(1)$ \\
$\mathrm{O}(2)$ & $8069(3)$ & $5222(1)$ & $1294(1)$ & $31(1)$ \\
$\mathrm{N}(1)$ & $2118(4)$ & $4590(1)$ & $1857(1)$ & $25(1)$ \\
$\mathrm{C}(1)$ & $3501(4)$ & $4287(1)$ & $1395(1)$ & $23(1)$ \\
$\mathrm{C}(2)$ & $2605(4)$ & $3571(1)$ & $1142(1)$ & $25(1)$ \\
$\mathrm{C}(3)$ & $3851(4)$ & $2933(1)$ & $1180(1)$ & $26(1)$ \\
$\mathrm{C}(4)$ & $3053(4)$ & $2254(1)$ & $951(1)$ & $29(1)$ \\
$\mathrm{C}(5)$ & $4308(5)$ & $1586(1)$ & $997(1)$ & $40(1)$ \\
$\mathrm{C}(6)$ & $3501(6)$ & $944(1)$ & $774(1)$ & $49(1)$ \\
$\mathrm{C}(7)$ & $1412(6)$ & $932(1)$ & $490(1)$ & $45(1)$ \\
$\mathrm{C}(8)$ & $157(5)$ & $1564(1)$ & $435(1)$ & $38(1)$
\end{tabular}




\begin{tabular}{lrrrr}
$\mathrm{C}(9)$ & $940(5)$ & $2243(1)$ & $667(1)$ & $29(1)$ \\
$\mathrm{C}(10)$ & $-304(5)$ & $2912(1)$ & $628(1)$ & $34(1)$ \\
$\mathrm{C}(11)$ & $487(4)$ & $3554(1)$ & $861(1)$ & $32(1)$ \\
$\mathrm{C}(12)$ & $3757(4)$ & $4893(1)$ & $941(1)$ & $26(1)$ \\
$\mathrm{C}(13)$ & $882(5)$ & $4743(1)$ & $2932(1)$ & $33(1)$ \\
$\mathrm{C}(14)$ & $2030(6)$ & $5494(2)$ & $2905(1)$ & $49(1)$ \\
$\mathrm{C}(15)$ & $-1615(5)$ & $4787(2)$ & $2815(1)$ & $53(1)$ \\
$\mathrm{C}(16)$ & $1321(7)$ & $4383(2)$ & $3508(1)$ & $68(1)$ \\
$\mathrm{C}(17)$ & $6400(4)$ & $6043(1)$ & $426(1)$ & $27(1)$ \\
$\mathrm{C}(18)$ & $8375(5)$ & $5873(2)$ & $139(1)$ & $40(1)$ \\
$\mathrm{C}(19)$ & $8863(6)$ & $6211(2)$ & $-370(1)$ & $49(1)$ \\
$\mathrm{C}(20)$ & $7367(7)$ & $6714(2)$ & $-594(1)$ & $50(1)$ \\
$\mathrm{C}(21)$ & $5393(7)$ & $6882(2)$ & $-318(1)$ & $54(1)$ \\
$\mathrm{C}(22)$ & $4916(5)$ & $6550(1)$ & $193(1)$ & $42(1)$ \\
$\mathrm{C}(23)$ & $4852(4)$ & $6262(1)$ & $1571(1)$ & $25(1)$ \\
$\mathrm{C}(24)$ & $6349(5)$ & $6524(1)$ & $1974(1)$ & $35(1)$ \\
$\mathrm{C}(25)$ & $5711(5)$ & $7086(2)$ & $2339(1)$ & $46(1)$ \\
$\mathrm{C}(26)$ & $3575(5)$ & $7394(1)$ & $2297(1)$ & $42(1)$ \\
$\mathrm{C}(27)$ & $2059(5)$ & $7130(1)$ & $1902(1)$ & $37(1)$ \\
$\mathrm{C}(28)$ & $2668(4)$ & $6563(1)$ & $1542(1)$ & $31(1)$ \\
\hline
\end{tabular}

Table 3. Bond lengths $[\AA]$ and angles $\left[{ }^{\circ}\right]$ for compound $\mathbf{1 7}(\boldsymbol{R}, \boldsymbol{S})$.

\begin{tabular}{ll}
\hline $\mathrm{S}(1)-\mathrm{O}(1)$ & $1.475(2)$ \\
$\mathrm{S}(1)-\mathrm{N}(1)$ & $1.662(2)$ \\
$\mathrm{S}(1)-\mathrm{C}(13)$ & $1.846(3)$ \\
$\mathrm{P}(1)-\mathrm{O}(2)$ & $1.4866(17)$ \\
$\mathrm{P}(1)-\mathrm{C}(23)$ & $1.799(2)$ \\
$\mathrm{P}(1)-\mathrm{C}(12)$ & $1.803(2)$ \\
$\mathrm{P}(1)-\mathrm{C}(17)$ & $1.817(3)$ \\
$\mathrm{N}(1)-\mathrm{C}(1)$ & $1.472(3)$ \\
$\mathrm{N}(1)-\mathrm{H}(1 \mathrm{~N})$ & $0.873(17)$ \\
$\mathrm{C}(1)-\mathrm{C}(2)$ & $1.516(3)$ \\
$\mathrm{C}(1)-\mathrm{C}(12)$ & $1.541(3)$ \\
$\mathrm{C}(1)-\mathrm{H}(1)$ & 0.9900 \\
$\mathrm{C}(2)-\mathrm{C}(3)$ & $1.364(3)$ \\
$\mathrm{C}(2)-\mathrm{C}(11)$ & $1.413(3)$
\end{tabular}




\begin{tabular}{|c|c|}
\hline $\mathrm{C}(3)-\mathrm{C}(4)$ & $1.417(3)$ \\
\hline $\mathrm{C}(3)-\mathrm{H}(3)$ & 0.9400 \\
\hline$C(4)-C(5)$ & $1.414(3)$ \\
\hline $\mathrm{C}(4)-\mathrm{C}(9)$ & $1.414(4)$ \\
\hline$C(5)-C(6)$ & $1.356(4)$ \\
\hline $\mathrm{C}(5)-\mathrm{H}(5)$ & 0.9400 \\
\hline$C(6)-C(7)$ & $1.401(5)$ \\
\hline $\mathrm{C}(6)-\mathrm{H}(6)$ & 0.9400 \\
\hline$C(7)-C(8)$ & $1.362(4)$ \\
\hline C(7)-H(7) & 0.9400 \\
\hline $\mathrm{C}(8)-\mathrm{C}(9)$ & $1.416(3)$ \\
\hline $\mathrm{C}(8)-\mathrm{H}(8)$ & 0.9400 \\
\hline C(9)-C(10) & $1.412(3)$ \\
\hline$C(10)-C(11)$ & $1.362(3)$ \\
\hline $\mathrm{C}(10)-\mathrm{H}(10)$ & 0.9400 \\
\hline $\mathrm{C}(11)-\mathrm{H}(11)$ & 0.9400 \\
\hline $\mathrm{C}(12)-\mathrm{H}(12 \mathrm{~A})$ & 0.9800 \\
\hline $\mathrm{C}(12)-\mathrm{H}(12 \mathrm{~B})$ & 0.9800 \\
\hline $\mathrm{C}(13)-\mathrm{C}(15)$ & $1.496(4)$ \\
\hline$C(13)-C(14)$ & $1.511(4)$ \\
\hline$C(13)-C(16)$ & $1.535(4)$ \\
\hline $\mathrm{C}(14)-\mathrm{H}(14 \mathrm{~A})$ & 0.9700 \\
\hline $\mathrm{C}(14)-\mathrm{H}(14 \mathrm{~B})$ & 0.9700 \\
\hline $\mathrm{C}(14)-\mathrm{H}(14 \mathrm{C})$ & 0.9700 \\
\hline $\mathrm{C}(15)-\mathrm{H}(15 \mathrm{~A})$ & 0.9700 \\
\hline $\mathrm{C}(15)-\mathrm{H}(15 \mathrm{~B})$ & 0.9700 \\
\hline $\mathrm{C}(15)-\mathrm{H}(15 \mathrm{C})$ & 0.9700 \\
\hline $\mathrm{C}(16)-\mathrm{H}(16 \mathrm{~A})$ & 0.9700 \\
\hline $\mathrm{C}(16)-\mathrm{H}(16 \mathrm{~B})$ & 0.9700 \\
\hline $\mathrm{C}(16)-\mathrm{H}(16 \mathrm{C})$ & 0.9700 \\
\hline $\mathrm{C}(17)-\mathrm{C}(22)$ & $1.378(4)$ \\
\hline $\mathrm{C}(17)-\mathrm{C}(18)$ & $1.381(4)$ \\
\hline C(18)-C(19) & $1.383(4)$ \\
\hline $\mathrm{C}(18)-\mathrm{H}(18)$ & 0.9400 \\
\hline$C(19)-C(20)$ & $1.370(5)$ \\
\hline C(19)-H(19) & 0.9400 \\
\hline $\mathrm{C}(20)-\mathrm{C}(21)$ & $1.366(5)$ \\
\hline $\mathrm{C}(20)-\mathrm{H}(20)$ & 0.9400 \\
\hline
\end{tabular}




\begin{tabular}{|c|c|}
\hline $\mathrm{C}(21)-\mathrm{C}(22)$ & $1.384(4)$ \\
\hline $\mathrm{C}(21)-\mathrm{H}(21)$ & 0.9400 \\
\hline $\mathrm{C}(22)-\mathrm{H}(22)$ & 0.9400 \\
\hline $\mathrm{C}(23)-\mathrm{C}(24)$ & $1.383(4)$ \\
\hline $\mathrm{C}(23)-\mathrm{C}(28)$ & $1.395(3)$ \\
\hline$C(24)-C(25)$ & $1.384(4)$ \\
\hline $\mathrm{C}(24)-\mathrm{H}(24)$ & 0.9400 \\
\hline$C(25)-C(26)$ & $1.375(4)$ \\
\hline $\mathrm{C}(25)-\mathrm{H}(25)$ & 0.9400 \\
\hline$C(26)-C(27)$ & $1.378(4)$ \\
\hline $\mathrm{C}(26)-\mathrm{H}(26)$ & 0.9400 \\
\hline $\mathrm{C}(27)-\mathrm{C}(28)$ & $1.380(3)$ \\
\hline $\mathrm{C}(27)-\mathrm{H}(27)$ & 0.9400 \\
\hline $\mathrm{C}(28)-\mathrm{H}(28)$ & 0.9400 \\
\hline $\mathrm{O}(1)-\mathrm{S}(1)-\mathrm{N}(1)$ & $111.98(12)$ \\
\hline $\mathrm{O}(1)-\mathrm{S}(1)-\mathrm{C}(13)$ & $104.63(12)$ \\
\hline $\mathrm{N}(1)-\mathrm{S}(1)-\mathrm{C}(13)$ & $99.04(11)$ \\
\hline $\mathrm{O}(2)-\mathrm{P}(1)-\mathrm{C}(23)$ & $112.70(11)$ \\
\hline $\mathrm{O}(2)-\mathrm{P}(1)-\mathrm{C}(12)$ & $112.07(10)$ \\
\hline $\mathrm{C}(23)-\mathrm{P}(1)-\mathrm{C}(12)$ & $110.20(11)$ \\
\hline $\mathrm{O}(2)-\mathrm{P}(1)-\mathrm{C}(17)$ & $110.40(11)$ \\
\hline $\mathrm{C}(23)-\mathrm{P}(1)-\mathrm{C}(17)$ & $106.50(11)$ \\
\hline $\mathrm{C}(12)-\mathrm{P}(1)-\mathrm{C}(17)$ & $104.50(11)$ \\
\hline $\mathrm{C}(1)-\mathrm{N}(1)-\mathrm{S}(1)$ & $113.40(15)$ \\
\hline $\mathrm{C}(1)-\mathrm{N}(1)-\mathrm{H}(1 \mathrm{~N})$ & $110.1(18)$ \\
\hline $\mathrm{S}(1)-\mathrm{N}(1)-\mathrm{H}(1 \mathrm{~N})$ & $114.5(17)$ \\
\hline $\mathrm{N}(1)-\mathrm{C}(1)-\mathrm{C}(2)$ & $114.77(19)$ \\
\hline $\mathrm{N}(1)-\mathrm{C}(1)-\mathrm{C}(12)$ & 108.34(17) \\
\hline $\mathrm{C}(2)-\mathrm{C}(1)-\mathrm{C}(12)$ & $110.99(19)$ \\
\hline $\mathrm{N}(1)-\mathrm{C}(1)-\mathrm{H}(1)$ & 107.5 \\
\hline $\mathrm{C}(2)-\mathrm{C}(1)-\mathrm{H}(1)$ & 107.5 \\
\hline $\mathrm{C}(12)-\mathrm{C}(1)-\mathrm{H}(1)$ & 107.5 \\
\hline $\mathrm{C}(3)-\mathrm{C}(2)-\mathrm{C}(11)$ & 119.1(2) \\
\hline$C(3)-C(2)-C(1)$ & $120.1(2)$ \\
\hline $\mathrm{C}(11)-\mathrm{C}(2)-\mathrm{C}(1)$ & $120.8(2)$ \\
\hline $\mathrm{C}(2)-\mathrm{C}(3)-\mathrm{C}(4)$ & $121.5(2)$ \\
\hline $\mathrm{C}(2)-\mathrm{C}(3)-\mathrm{H}(3)$ & 119.3 \\
\hline
\end{tabular}




\begin{tabular}{|c|c|}
\hline $\mathrm{C}(4)-\mathrm{C}(3)-\mathrm{H}(3)$ & 119.3 \\
\hline$C(5)-C(4)-C(9)$ & $118.9(2)$ \\
\hline$C(5)-C(4)-C(3)$ & $122.0(2)$ \\
\hline $\mathrm{C}(9)-\mathrm{C}(4)-\mathrm{C}(3)$ & $119.1(2)$ \\
\hline$C(6)-C(5)-C(4)$ & $120.7(3)$ \\
\hline $\mathrm{C}(6)-\mathrm{C}(5)-\mathrm{H}(5)$ & 119.6 \\
\hline $\mathrm{C}(4)-\mathrm{C}(5)-\mathrm{H}(5)$ & 119.6 \\
\hline$C(5)-C(6)-C(7)$ & $120.5(3)$ \\
\hline $\mathrm{C}(5)-\mathrm{C}(6)-\mathrm{H}(6)$ & 119.7 \\
\hline $\mathrm{C}(7)-\mathrm{C}(6)-\mathrm{H}(6)$ & 119.7 \\
\hline$C(8)-C(7)-C(6)$ & $120.5(3)$ \\
\hline $\mathrm{C}(8)-\mathrm{C}(7)-\mathrm{H}(7)$ & 119.8 \\
\hline $\mathrm{C}(6)-\mathrm{C}(7)-\mathrm{H}(7)$ & 119.8 \\
\hline$C(7)-C(8)-C(9)$ & $120.5(3)$ \\
\hline $\mathrm{C}(7)-\mathrm{C}(8)-\mathrm{H}(8)$ & 119.8 \\
\hline $\mathrm{C}(9)-\mathrm{C}(8)-\mathrm{H}(8)$ & 119.8 \\
\hline $\mathrm{C}(10)-\mathrm{C}(9)-\mathrm{C}(4)$ & $118.3(2)$ \\
\hline$C(10)-C(9)-C(8)$ & $122.8(3)$ \\
\hline $\mathrm{C}(4)-\mathrm{C}(9)-\mathrm{C}(8)$ & $118.9(2)$ \\
\hline $\mathrm{C}(11)-\mathrm{C}(10)-\mathrm{C}(9)$ & 121.3(2) \\
\hline $\mathrm{C}(11)-\mathrm{C}(10)-\mathrm{H}(10)$ & 119.3 \\
\hline $\mathrm{C}(9)-\mathrm{C}(10)-\mathrm{H}(10)$ & 119.3 \\
\hline$C(10)-C(11)-C(2)$ & $120.7(2)$ \\
\hline $\mathrm{C}(10)-\mathrm{C}(11)-\mathrm{H}(11)$ & 119.7 \\
\hline $\mathrm{C}(2)-\mathrm{C}(11)-\mathrm{H}(11)$ & 119.7 \\
\hline $\mathrm{C}(1)-\mathrm{C}(12)-\mathrm{P}(1)$ & $114.20(16)$ \\
\hline $\mathrm{C}(1)-\mathrm{C}(12)-\mathrm{H}(12 \mathrm{~A})$ & 108.7 \\
\hline $\mathrm{P}(1)-\mathrm{C}(12)-\mathrm{H}(12 \mathrm{~A})$ & 108.7 \\
\hline $\mathrm{C}(1)-\mathrm{C}(12)-\mathrm{H}(12 \mathrm{~B})$ & 108.7 \\
\hline $\mathrm{P}(1)-\mathrm{C}(12)-\mathrm{H}(12 \mathrm{~B})$ & 108.7 \\
\hline $\mathrm{H}(12 \mathrm{~A})-\mathrm{C}(12)-\mathrm{H}(12 \mathrm{~B})$ & 107.6 \\
\hline $\mathrm{C}(15)-\mathrm{C}(13)-\mathrm{C}(14)$ & $112.5(3)$ \\
\hline $\mathrm{C}(15)-\mathrm{C}(13)-\mathrm{C}(16)$ & $110.6(3)$ \\
\hline $\mathrm{C}(14)-\mathrm{C}(13)-\mathrm{C}(16)$ & $109.9(3)$ \\
\hline$C(15)-C(13)-S(1)$ & $110.0(2)$ \\
\hline $\mathrm{C}(14)-\mathrm{C}(13)-\mathrm{S}(1)$ & $110.68(19)$ \\
\hline $\mathrm{C}(16)-\mathrm{C}(13)-\mathrm{S}(1)$ & $102.64(19)$ \\
\hline $\mathrm{C}(13)-\mathrm{C}(14)-\mathrm{H}(14 \mathrm{~A})$ & 109.5 \\
\hline
\end{tabular}




\begin{tabular}{|c|c|}
\hline $\mathrm{C}(13)-\mathrm{C}(14)-\mathrm{H}(14 \mathrm{~B})$ & 109.5 \\
\hline $\mathrm{H}(14 \mathrm{~A})-\mathrm{C}(14)-\mathrm{H}(14 \mathrm{~B})$ & 109.5 \\
\hline $\mathrm{C}(13)-\mathrm{C}(14)-\mathrm{H}(14 \mathrm{C})$ & 109.5 \\
\hline $\mathrm{H}(14 \mathrm{~A})-\mathrm{C}(14)-\mathrm{H}(14 \mathrm{C})$ & 109.5 \\
\hline $\mathrm{H}(14 \mathrm{~B})-\mathrm{C}(14)-\mathrm{H}(14 \mathrm{C})$ & 109.5 \\
\hline $\mathrm{C}(13)-\mathrm{C}(15)-\mathrm{H}(15 \mathrm{~A})$ & 109.5 \\
\hline $\mathrm{C}(13)-\mathrm{C}(15)-\mathrm{H}(15 \mathrm{~B})$ & 109.5 \\
\hline $\mathrm{H}(15 \mathrm{~A})-\mathrm{C}(15)-\mathrm{H}(15 \mathrm{~B})$ & 109.5 \\
\hline $\mathrm{C}(13)-\mathrm{C}(15)-\mathrm{H}(15 \mathrm{C})$ & 109.5 \\
\hline $\mathrm{H}(15 \mathrm{~A})-\mathrm{C}(15)-\mathrm{H}(15 \mathrm{C})$ & 109.5 \\
\hline$H(15 B)-C(15)-H(15 C)$ & 109.5 \\
\hline $\mathrm{C}(13)-\mathrm{C}(16)-\mathrm{H}(16 \mathrm{~A})$ & 109.5 \\
\hline $\mathrm{C}(13)-\mathrm{C}(16)-\mathrm{H}(16 \mathrm{~B})$ & 109.5 \\
\hline$H(16 A)-C(16)-H(16 B)$ & 109.5 \\
\hline$C(13)-C(16)-H(16 C)$ & 109.5 \\
\hline$H(16 A)-C(16)-H(16 C)$ & 109.5 \\
\hline$H(16 B)-C(16)-H(16 C)$ & 109.5 \\
\hline$C(22)-C(17)-C(18)$ & $118.7(2)$ \\
\hline $\mathrm{C}(22)-\mathrm{C}(17)-\mathrm{P}(1)$ & $124.2(2)$ \\
\hline $\mathrm{C}(18)-\mathrm{C}(17)-\mathrm{P}(1)$ & $117.2(2)$ \\
\hline$C(19)-C(18)-C(17)$ & $120.6(3)$ \\
\hline $\mathrm{C}(19)-\mathrm{C}(18)-\mathrm{H}(18)$ & 119.7 \\
\hline $\mathrm{C}(17)-\mathrm{C}(18)-\mathrm{H}(18)$ & 119.7 \\
\hline$C(20)-C(19)-C(18)$ & $119.8(3)$ \\
\hline $\mathrm{C}(20)-\mathrm{C}(19)-\mathrm{H}(19)$ & 120.1 \\
\hline $\mathrm{C}(18)-\mathrm{C}(19)-\mathrm{H}(19)$ & 120.1 \\
\hline$C(21)-C(20)-C(19)$ & $120.3(3)$ \\
\hline $\mathrm{C}(21)-\mathrm{C}(20)-\mathrm{H}(20)$ & 119.9 \\
\hline $\mathrm{C}(19)-\mathrm{C}(20)-\mathrm{H}(20)$ & 119.9 \\
\hline$C(20)-C(21)-C(22)$ & $119.8(3)$ \\
\hline $\mathrm{C}(20)-\mathrm{C}(21)-\mathrm{H}(21)$ & 120.1 \\
\hline $\mathrm{C}(22)-\mathrm{C}(21)-\mathrm{H}(21)$ & 120.1 \\
\hline$C(17)-C(22)-C(21)$ & $120.7(3)$ \\
\hline $\mathrm{C}(17)-\mathrm{C}(22)-\mathrm{H}(22)$ & 119.6 \\
\hline $\mathrm{C}(21)-\mathrm{C}(22)-\mathrm{H}(22)$ & 119.6 \\
\hline $\mathrm{C}(24)-\mathrm{C}(23)-\mathrm{C}(28)$ & $119.2(2)$ \\
\hline $\mathrm{C}(24)-\mathrm{C}(23)-\mathrm{P}(1)$ & $116.74(19)$ \\
\hline $\mathrm{C}(28)-\mathrm{C}(23)-\mathrm{P}(1)$ & $124.0(2)$ \\
\hline
\end{tabular}




$\begin{array}{ll}\mathrm{C}(23)-\mathrm{C}(24)-\mathrm{C}(25) & 120.7(3) \\ \mathrm{C}(23)-\mathrm{C}(24)-\mathrm{H}(24) & 119.6 \\ \mathrm{C}(25)-\mathrm{C}(24)-\mathrm{H}(24) & 119.6 \\ \mathrm{C}(26)-\mathrm{C}(25)-\mathrm{C}(24) & 119.7(3) \\ \mathrm{C}(26)-\mathrm{C}(25)-\mathrm{H}(25) & 120.1 \\ \mathrm{C}(24)-\mathrm{C}(25)-\mathrm{H}(25) & 120.1 \\ \mathrm{C}(25)-\mathrm{C}(26)-\mathrm{C}(27) & 120.0(2) \\ \mathrm{C}(25)-\mathrm{C}(26)-\mathrm{H}(26) & 120.0 \\ \mathrm{C}(27)-\mathrm{C}(26)-\mathrm{H}(26) & 120.0 \\ \mathrm{C}(26)-\mathrm{C}(27)-\mathrm{C}(28) & 120.7(3) \\ \mathrm{C}(26)-\mathrm{C}(27)-\mathrm{H}(27) & 119.7 \\ \mathrm{C}(28)-\mathrm{C}(27)-\mathrm{H}(27) & 119.7 \\ \mathrm{C}(27)-\mathrm{C}(28)-\mathrm{C}(23) & 119.6(3) \\ \mathrm{C}(27)-\mathrm{C}(28)-\mathrm{H}(28) & 120.2 \\ \mathrm{C}(23)-\mathrm{C}(28)-\mathrm{H}(28) & 120.2\end{array}$

Symmetry transformations used to generate equivalent atoms:

Table 4. Anisotropic displacement parameters $\left(\AA^{2} \times 10^{3}\right)$ for compound $\mathbf{1 7}(\boldsymbol{R}, \boldsymbol{S})$.

The anisotropic displacement factor exponent takes the form: $-2 \pi^{2}\left[h^{2} a^{* 2} U^{11}+\ldots+2 h k\right.$ $a^{*} b^{*} U^{12}$ ]

\begin{tabular}{|c|c|c|c|c|c|c|}
\hline & $\mathrm{U}^{11}$ & $\mathrm{U}^{22}$ & $\mathrm{U}^{33}$ & $\mathrm{U}^{23}$ & $\mathrm{U}^{13}$ & $\mathrm{U}^{12}$ \\
\hline$S(1)$ & $35(1)$ & $30(1)$ & $31(1)$ & $9(1)$ & $4(1)$ & $5(1)$ \\
\hline $\mathrm{P}(1)$ & $22(1)$ & $22(1)$ & $25(1)$ & $1(1)$ & $1(1)$ & $1(1)$ \\
\hline $\mathrm{O}(1)$ & $73(2)$ & $26(1)$ & $60(1)$ & $6(1)$ & $24(1)$ & $-7(1)$ \\
\hline $\mathrm{O}(2)$ & $28(1)$ & $34(1)$ & $32(1)$ & $1(1)$ & $-2(1)$ & $6(1)$ \\
\hline $\mathrm{N}(1)$ & $25(1)$ & $25(1)$ & $24(1)$ & $2(1)$ & $-1(1)$ & $1(1)$ \\
\hline $\mathrm{C}(1)$ & $21(1)$ & $25(1)$ & $24(2)$ & $1(1)$ & $-1(1)$ & $1(1)$ \\
\hline$C(2)$ & $28(1)$ & $23(1)$ & $24(1)$ & $2(1)$ & $3(1)$ & $-2(1)$ \\
\hline $\mathrm{C}(3)$ & $23(1)$ & $28(1)$ & $26(2)$ & $2(1)$ & $-1(1)$ & $2(1)$ \\
\hline$C(4)$ & $34(1)$ & $26(1)$ & $26(2)$ & $2(1)$ & $4(1)$ & $-3(1)$ \\
\hline$C(5)$ & $47(2)$ & $32(1)$ & $42(2)$ & $-2(1)$ & $-6(1)$ & $6(1)$ \\
\hline$C(6)$ & $74(2)$ & $24(1)$ & $47(2)$ & $-2(1)$ & $2(2)$ & $6(1)$ \\
\hline$C(7)$ & $70(2)$ & $27(1)$ & $38(2)$ & $-6(1)$ & $3(2)$ & $-8(1)$ \\
\hline $\mathrm{C}(8)$ & $45(2)$ & $36(1)$ & $32(2)$ & $-5(1)$ & $-1(1)$ & $-10(1)$ \\
\hline $\mathrm{C}(9)$ & $37(2)$ & $28(1)$ & $23(2)$ & $0(1)$ & $6(1)$ & $-4(1)$ \\
\hline
\end{tabular}




\begin{tabular}{lllllll}
$\mathrm{C}(10)$ & $25(1)$ & $37(1)$ & $39(2)$ & $-5(1)$ & $-5(1)$ & $-1(1)$ \\
$\mathrm{C}(11)$ & $29(2)$ & $28(1)$ & $40(2)$ & $-4(1)$ & $-3(1)$ & $4(1)$ \\
$\mathrm{C}(12)$ & $32(1)$ & $23(1)$ & $22(2)$ & $-2(1)$ & $-2(1)$ & $-2(1)$ \\
$\mathrm{C}(13)$ & $33(2)$ & $40(1)$ & $27(2)$ & $4(1)$ & $4(1)$ & $0(1)$ \\
$\mathrm{C}(14)$ & $54(2)$ & $46(2)$ & $47(2)$ & $-17(1)$ & $8(2)$ & $-12(2)$ \\
$\mathrm{C}(15)$ & $37(2)$ & $67(2)$ & $56(2)$ & $-12(2)$ & $7(2)$ & $7(2)$ \\
$\mathrm{C}(16)$ & $95(3)$ & $80(2)$ & $29(2)$ & $13(2)$ & $6(2)$ & $20(2)$ \\
$\mathrm{C}(17)$ & $31(1)$ & $24(1)$ & $27(2)$ & $2(1)$ & $2(1)$ & $-6(1)$ \\
$\mathrm{C}(18)$ & $35(2)$ & $47(2)$ & $36(2)$ & $4(1)$ & $4(1)$ & $1(1)$ \\
$\mathrm{C}(19)$ & $56(2)$ & $59(2)$ & $32(2)$ & $2(1)$ & $13(2)$ & $-9(2)$ \\
$\mathrm{C}(20)$ & $79(3)$ & $41(2)$ & $31(2)$ & $5(1)$ & $4(2)$ & $-18(2)$ \\
$\mathrm{C}(21)$ & $77(3)$ & $42(2)$ & $42(2)$ & $14(1)$ & $-4(2)$ & $4(2)$ \\
$\mathrm{C}(22)$ & $48(2)$ & $38(1)$ & $40(2)$ & $11(1)$ & $6(1)$ & $8(1)$ \\
$\mathrm{C}(23)$ & $25(1)$ & $24(1)$ & $26(2)$ & $3(1)$ & $5(1)$ & $0(1)$ \\
$\mathrm{C}(24)$ & $27(2)$ & $35(1)$ & $42(2)$ & $-9(1)$ & $0(1)$ & $-3(1)$ \\
$\mathrm{C}(25)$ & $47(2)$ & $41(2)$ & $50(2)$ & $-15(1)$ & $-1(2)$ & $-10(1)$ \\
$\mathrm{C}(26)$ & $52(2)$ & $26(1)$ & $48(2)$ & $-11(1)$ & $16(2)$ & $-1(1)$ \\
$\mathrm{C}(27)$ & $34(2)$ & $27(1)$ & $49(2)$ & $-1(1)$ & $13(1)$ & $6(1)$ \\
$\mathrm{C}(28)$ & $27(1)$ & $31(1)$ & $36(2)$ & $4(1)$ & $2(1)$ & $0(1)$ \\
\hline & & & & & \\
\hline
\end{tabular}

Table 5. Hydrogen coordinates $\left(\times 10^{4}\right)$ and isotropic displacement parameters $\left(\AA^{2} \times 10^{3}\right)$ for compound $17(R, S)$.

\begin{tabular}{|c|c|c|c|c|}
\hline & $\mathrm{x}$ & $\mathrm{y}$ & $\mathrm{z}$ & $\mathrm{U}(\mathrm{eq})$ \\
\hline $\mathrm{H}(1 \mathrm{~N})$ & $740(30)$ & $4683(13)$ & $1740(11)$ & 30 \\
\hline $\mathrm{H}(1)$ & 5036 & 4182 & 1547 & 28 \\
\hline $\mathrm{H}(3)$ & 5270 & 2944 & 1362 & 31 \\
\hline $\mathrm{H}(5)$ & 5717 & 1587 & 1184 & 48 \\
\hline $\mathrm{H}(6)$ & 4351 & 504 & 810 & 58 \\
\hline $\mathrm{H}(7)$ & 874 & 485 & 335 & 54 \\
\hline $\mathrm{H}(8)$ & -1240 & 1550 & 243 & 45 \\
\hline $\mathrm{H}(10)$ & -1707 & 2915 & 438 & 40 \\
\hline $\mathrm{H}(11)$ & -386 & 3990 & 835 & 39 \\
\hline $\mathrm{H}(12 \mathrm{~A})$ & 2297 & 5150 & 898 & 31 \\
\hline
\end{tabular}




\begin{tabular}{lrrrr}
$\mathrm{H}(12 \mathrm{~B})$ & 4113 & 4654 & 581 & 31 \\
$\mathrm{H}(14 \mathrm{~A})$ & 1651 & 5735 & 2552 & 74 \\
$\mathrm{H}(14 \mathrm{~B})$ & 3666 & 5430 & 2930 & 74 \\
$\mathrm{H}(14 \mathrm{C})$ & 1511 & 5800 & 3216 & 74 \\
$\mathrm{H}(15 \mathrm{~A})$ & -2339 & 5101 & 3095 & 80 \\
$\mathrm{H}(15 \mathrm{~B})$ & -2269 & 4293 & 2831 & 80 \\
$\mathrm{H}(15 \mathrm{C})$ & -1858 & 4996 & 2444 & 80 \\
$\mathrm{H}(16 \mathrm{~A})$ & 683 & 4694 & 3801 & 102 \\
$\mathrm{H}(16 \mathrm{~B})$ & 2947 & 4332 & 3566 & 102 \\
$\mathrm{H}(16 \mathrm{C})$ & 611 & 3897 & 3519 & 102 \\
$\mathrm{H}(18)$ & 9397 & 5524 & 290 & 47 \\
$\mathrm{H}(19)$ & 10218 & 6095 & -561 & 59 \\
$\mathrm{H}(20)$ & 7699 & 6944 & -940 & 60 \\
$\mathrm{H}(21)$ & 4362 & 7223 & -475 & 64 \\
$\mathrm{H}(22)$ & 3565 & 6671 & 384 & 50 \\
$\mathrm{H}(24)$ & 7815 & 6318 & 2000 & 41 \\
$\mathrm{H}(25)$ & 6733 & 7257 & 2614 & 55 \\
$\mathrm{H}(26)$ & 3149 & 7784 & 2538 & 51 \\
$\mathrm{H}(27)$ & 596 & 7339 & 1879 & 44 \\
$\mathrm{H}(28)$ & 1616 & 6380 & 1278 & 38 \\
\hline
\end{tabular}

Table 6. Torsion angles $\left[{ }^{\circ}\right]$ for $\mathbf{1 7}(\boldsymbol{R}, S)$.

\begin{tabular}{lc}
\hline $\mathrm{O}(1)-\mathrm{S}(1)-\mathrm{N}(1)-\mathrm{C}(1)$ & $-82.00(18)$ \\
$\mathrm{C}(13)-\mathrm{S}(1)-\mathrm{N}(1)-\mathrm{C}(1)$ & $168.13(17)$ \\
$\mathrm{S}(1)-\mathrm{N}(1)-\mathrm{C}(1)-\mathrm{C}(2)$ & $70.1(2)$ \\
$\mathrm{S}(1)-\mathrm{N}(1)-\mathrm{C}(1)-\mathrm{C}(12)$ & $-165.25(15)$ \\
$\mathrm{N}(1)-\mathrm{C}(1)-\mathrm{C}(2)-\mathrm{C}(3)$ & $-116.3(2)$ \\
$\mathrm{C}(12)-\mathrm{C}(1)-\mathrm{C}(2)-\mathrm{C}(3)$ & $120.5(2)$ \\
$\mathrm{N}(1)-\mathrm{C}(1)-\mathrm{C}(2)-\mathrm{C}(11)$ & $64.2(3)$ \\
$\mathrm{C}(12)-\mathrm{C}(1)-\mathrm{C}(2)-\mathrm{C}(11)$ & $-59.1(3)$ \\
$\mathrm{C}(11)-\mathrm{C}(2)-\mathrm{C}(3)-\mathrm{C}(4)$ & $-0.8(4)$ \\
$\mathrm{C}(1)-\mathrm{C}(2)-\mathrm{C}(3)-\mathrm{C}(4)$ & $179.6(2)$ \\
$\mathrm{C}(2)-\mathrm{C}(3)-\mathrm{C}(4)-\mathrm{C}(5)$ & $-178.8(2)$ \\
$\mathrm{C}(2)-\mathrm{C}(3)-\mathrm{C}(4)-\mathrm{C}(9)$ & $1.3(4)$ \\
$\mathrm{C}(9)-\mathrm{C}(4)-\mathrm{C}(5)-\mathrm{C}(6)$ & $-0.1(4)$ \\
$\mathrm{C}(3)-\mathrm{C}(4)-\mathrm{C}(5)-\mathrm{C}(6)$ & $179.9(3)$
\end{tabular}




\begin{tabular}{|c|c|}
\hline$C(4)-C(5)-C(6)-C(7)$ & $0.4(5)$ \\
\hline$C(5)-C(6)-C(7)-C(8)$ & $-0.3(5)$ \\
\hline $\mathrm{C}(6)-\mathrm{C}(7)-\mathrm{C}(8)-\mathrm{C}(9)$ & $-0.2(4)$ \\
\hline$C(5)-C(4)-C(9)-C(10)$ & $179.5(3)$ \\
\hline $\mathrm{C}(3)-\mathrm{C}(4)-\mathrm{C}(9)-\mathrm{C}(10)$ & $-0.6(4)$ \\
\hline$C(5)-C(4)-C(9)-C(8)$ & $-0.3(4)$ \\
\hline$C(3)-C(4)-C(9)-C(8)$ & $179.6(2)$ \\
\hline$C(7)-C(8)-C(9)-C(10)$ & $-179.3(3)$ \\
\hline$C(7)-C(8)-C(9)-C(4)$ & $0.5(4)$ \\
\hline$C(4)-C(9)-C(10)-C(11)$ & $-0.5(4)$ \\
\hline $\mathrm{C}(8)-\mathrm{C}(9)-\mathrm{C}(10)-\mathrm{C}(11)$ & $179.3(3)$ \\
\hline$C(9)-C(10)-C(11)-C(2)$ & $1.0(4)$ \\
\hline $\mathrm{C}(3)-\mathrm{C}(2)-\mathrm{C}(11)-\mathrm{C}(10)$ & $-0.3(4)$ \\
\hline$C(1)-C(2)-C(11)-C(10)$ & $179.2(2)$ \\
\hline $\mathrm{N}(1)-\mathrm{C}(1)-\mathrm{C}(12)-\mathrm{P}(1)$ & $80.8(2)$ \\
\hline $\mathrm{C}(2)-\mathrm{C}(1)-\mathrm{C}(12)-\mathrm{P}(1)$ & $-152.31(16)$ \\
\hline $\mathrm{O}(2)-\mathrm{P}(1)-\mathrm{C}(12)-\mathrm{C}(1)$ & $45.1(2)$ \\
\hline $\mathrm{C}(23)-\mathrm{P}(1)-\mathrm{C}(12)-\mathrm{C}(1)$ & $-81.25(19)$ \\
\hline $\mathrm{C}(17)-\mathrm{P}(1)-\mathrm{C}(12)-\mathrm{C}(1)$ & $164.68(17)$ \\
\hline $\mathrm{O}(1)-\mathrm{S}(1)-\mathrm{C}(13)-\mathrm{C}(15)$ & $-43.4(2)$ \\
\hline$N(1)-S(1)-C(13)-C(15)$ & $72.3(2)$ \\
\hline $\mathrm{O}(1)-\mathrm{S}(1)-\mathrm{C}(13)-\mathrm{C}(14)$ & $-168.4(2)$ \\
\hline$N(1)-S(1)-C(13)-C(14)$ & $-52.7(2)$ \\
\hline $\mathrm{O}(1)-\mathrm{S}(1)-\mathrm{C}(13)-\mathrm{C}(16)$ & $74.4(2)$ \\
\hline$N(1)-S(1)-C(13)-C(16)$ & $-169.9(2)$ \\
\hline $\mathrm{O}(2)-\mathrm{P}(1)-\mathrm{C}(17)-\mathrm{C}(22)$ & $-165.2(2)$ \\
\hline $\mathrm{C}(23)-\mathrm{P}(1)-\mathrm{C}(17)-\mathrm{C}(22)$ & $-42.6(2)$ \\
\hline $\mathrm{C}(12)-\mathrm{P}(1)-\mathrm{C}(17)-\mathrm{C}(22)$ & $74.1(2)$ \\
\hline $\mathrm{O}(2)-\mathrm{P}(1)-\mathrm{C}(17)-\mathrm{C}(18)$ & $15.1(2)$ \\
\hline $\mathrm{C}(23)-\mathrm{P}(1)-\mathrm{C}(17)-\mathrm{C}(18)$ & $137.7(2)$ \\
\hline $\mathrm{C}(12)-\mathrm{P}(1)-\mathrm{C}(17)-\mathrm{C}(18)$ & $-105.6(2)$ \\
\hline $\mathrm{C}(22)-\mathrm{C}(17)-\mathrm{C}(18)-\mathrm{C}(19)$ & $0.7(4)$ \\
\hline$P(1)-C(17)-C(18)-C(19)$ & $-179.6(2)$ \\
\hline $\mathrm{C}(17)-\mathrm{C}(18)-\mathrm{C}(19)-\mathrm{C}(20)$ & $-0.6(4)$ \\
\hline $\mathrm{C}(18)-\mathrm{C}(19)-\mathrm{C}(20)-\mathrm{C}(21)$ & $-0.1(5)$ \\
\hline $\mathrm{C}(19)-\mathrm{C}(20)-\mathrm{C}(21)-\mathrm{C}(22)$ & $0.7(5)$ \\
\hline $\mathrm{C}(18)-\mathrm{C}(17)-\mathrm{C}(22)-\mathrm{C}(21)$ & $0.0(4)$ \\
\hline$P(1)-C(17)-C(22)-C(21)$ & $-179.7(2)$ \\
\hline
\end{tabular}




$\begin{array}{lc}\mathrm{C}(20)-\mathrm{C}(21)-\mathrm{C}(22)-\mathrm{C}(17) & -0.7(5) \\ \mathrm{O}(2)-\mathrm{P}(1)-\mathrm{C}(23)-\mathrm{C}(24) & 16.3(2) \\ \mathrm{C}(12)-\mathrm{P}(1)-\mathrm{C}(23)-\mathrm{C}(24) & 142.34(19) \\ \mathrm{C}(17)-\mathrm{P}(1)-\mathrm{C}(23)-\mathrm{C}(24) & -104.9(2) \\ \mathrm{O}(2)-\mathrm{P}(1)-\mathrm{C}(23)-\mathrm{C}(28) & -166.54(18) \\ \mathrm{C}(12)-\mathrm{P}(1)-\mathrm{C}(23)-\mathrm{C}(28) & -40.5(2) \\ \mathrm{C}(17)-\mathrm{P}(1)-\mathrm{C}(23)-\mathrm{C}(28) & 72.3(2) \\ \mathrm{C}(28)-\mathrm{C}(23)-\mathrm{C}(24)-\mathrm{C}(25) & -1.1(4) \\ \mathrm{P}(1)-\mathrm{C}(23)-\mathrm{C}(24)-\mathrm{C}(25) & 176.2(2) \\ \mathrm{C}(23)-\mathrm{C}(24)-\mathrm{C}(25)-\mathrm{C}(26) & -0.7(4) \\ \mathrm{C}(24)-\mathrm{C}(25)-\mathrm{C}(26)-\mathrm{C}(27) & 1.6(4) \\ \mathrm{C}(25)-\mathrm{C}(26)-\mathrm{C}(27)-\mathrm{C}(28) & -0.7(4) \\ \mathrm{C}(26)-\mathrm{C}(27)-\mathrm{C}(28)-\mathrm{C}(23) & -1.1(4) \\ \mathrm{C}(24)-\mathrm{C}(23)-\mathrm{C}(28)-\mathrm{C}(27) & 1.9(3) \\ \mathrm{P}(1)-\mathrm{C}(23)-\mathrm{C}(28)-\mathrm{C}(27) & -175.12(19) \\ \end{array}$

Symmetry transformations used to generate equivalent atoms:

Table 7. Hydrogen bonds for compound $\mathbf{1 7}(R, S)$ [ $\AA^{\circ}$ and $^{\circ}$ ].

\begin{tabular}{lllll}
\hline D-H...A & $d(D-H)$ & $d(H \ldots A)$ & $d(D \ldots A)$ & $<($ DHA $)$ \\
\hline $\mathrm{N}(1)-H(1 \mathrm{~N}) \ldots \mathrm{O}(2) \# 1$ & $0.873(17)$ & $2.128(18)$ & $2.958(3)$ & $159(2)$ \\
\hline
\end{tabular}

Symmetry transformations used to generate equivalent atoms:

$\# 1 \mathrm{x}-1, \mathrm{y}, \mathrm{z}$ 

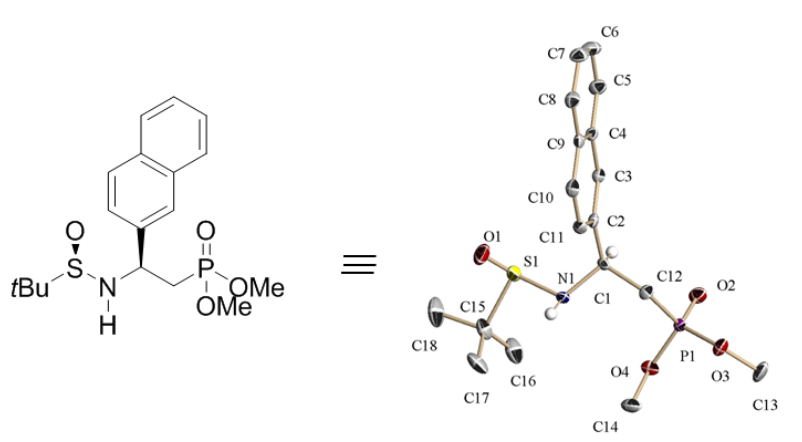

Table 1. Crystal data and structure refinement for compound $\mathbf{1 8}(R, S)$.

Empirical formula

Formula weight

Temperature

Wavelength

Crystal system

Space group

Unit cell dimensions

Volume

Z

Density (calculated)

Absorption coefficient

$\mathrm{F}(000)$

Crystal size

Theta range for data collection

Index ranges

Reflections collected

Independent reflections

Completeness to theta $=25.25^{\circ}$

Absorption correction

Max. and min. transmission

Refinement method

Data / restraints / parameters

Goodness-of-fit on $\mathrm{F}^{2}$

Final $\mathrm{R}$ indices $[\mathrm{I}>2 \operatorname{sigma}(\mathrm{I})]$
$\mathrm{C}_{18} \mathrm{H}_{26} \mathrm{NO}_{4} \mathrm{PS}$

383.43

213(2) K

$0.71073 \AA$

Orthorhombic

P $22_{1} 2_{1}$

$\mathrm{a}=5.8253(12) \AA \quad \alpha=90^{\circ}$.

$\mathrm{b}=17.338(3) \AA \quad \beta=90^{\circ}$.

$\mathrm{c}=19.996(3) \AA \quad \gamma=90^{\circ}$.

2019.5(6) $\AA^{3}$

4

$1.261 \mathrm{Mg} / \mathrm{m}^{3}$

$0.260 \mathrm{~mm}^{-1}$

816

$0.30 \times 0.10 \times 0.05 \mathrm{~mm}^{3}$

3.64 to $25.25^{\circ}$.

$-5<=\mathrm{h}<=6,-20<=\mathrm{k}<=8,-22<=1<=23$

10103

$3599[\mathrm{R}(\mathrm{int})=0.0720]$

$99.3 \%$

Semi-empirical from equivalents

0.9871 and 0.9690

Full-matrix least-squares on $\mathrm{F}^{2}$

3599 / 1 / 234

1.032

$\mathrm{R} 1=0.0657, \mathrm{wR} 2=0.1431$ 
$\mathrm{R}$ indices (all data)

Absolute structure parameter

Largest diff. peak and hole
$\mathrm{R} 1=0.0946, \mathrm{wR} 2=0.1533$

$-0.02(19)$

0.295 and -0.415 e. $\AA^{-3}$

Table 2. Atomic coordinates $\left(\times 10^{4}\right)$ and equivalent isotropic displacement parameters $\left(\AA^{2} \times 10^{3}\right)$ for compound $\mathbf{1 8}(\boldsymbol{R}, S)$. U(eq) is defined as one third of the trace of the orthogonalized $\mathrm{U}^{\mathrm{ij}}$ tensor.

\begin{tabular}{|c|c|c|c|c|}
\hline & $\mathrm{x}$ & $\mathrm{y}$ & $\mathrm{z}$ & $\mathrm{U}(\mathrm{eq})$ \\
\hline$S(1)$ & $150(4)$ & $2773(1)$ & $3112(1)$ & $46(1)$ \\
\hline $\mathrm{P}(1)$ & $4519(3)$ & $4866(1)$ & $2125(1)$ & $24(1)$ \\
\hline $\mathrm{O}(1)$ & $-1860(12)$ & $2332(2)$ & $2864(2)$ & $78(2)$ \\
\hline $\mathrm{O}(2)$ & $6771(8)$ & $4525(2)$ & $2184(2)$ & $40(1)$ \\
\hline $\mathrm{O}(3)$ & 4391(8) & $5578(2)$ & 1631(2) & $37(1)$ \\
\hline $\mathrm{O}(4)$ & $3675(7)$ & $5148(2)$ & $2828(2)$ & $33(1)$ \\
\hline $\mathrm{N}(1)$ & $587(10)$ & $3558(2)$ & $2661(2)$ & $26(1)$ \\
\hline $\mathrm{C}(1)$ & $2151(10)$ & $3454(3)$ & 2092(2) & $20(1)$ \\
\hline$C(2)$ & $1373(11)$ & $2860(3)$ & $1577(2)$ & $24(1)$ \\
\hline$C(3)$ & $2752(11)$ & $2252(3)$ & $1431(2)$ & $23(1)$ \\
\hline$C(4)$ & $2116(11)$ & 1701(3) & $927(3)$ & $23(1)$ \\
\hline$C(5)$ & $3535(13)$ & $1076(3)$ & $759(3)$ & $38(2)$ \\
\hline$C(6)$ & $2870(15)$ & $572(3)$ & $265(3)$ & $47(2)$ \\
\hline$C(7)$ & $835(15)$ & $669(3)$ & $-69(3)$ & $43(2)$ \\
\hline$C(8)$ & $-617(14)$ & 1261(3) & $89(2)$ & $34(2)$ \\
\hline $\mathrm{C}(9)$ & $23(11)$ & 1803(3) & $593(2)$ & $25(2)$ \\
\hline$C(10)$ & $-1399(12)$ & $2431(3)$ & $768(3)$ & $32(2)$ \\
\hline$C(11)$ & $-743(11)$ & $2949(3)$ & $1246(3)$ & $27(1)$ \\
\hline$C(12)$ & $2452(12)$ & $4241(3)$ & $1745(3)$ & $28(1)$ \\
\hline$C(13)$ & $6060(14)$ & $6194(3)$ & $1706(4)$ & $54(2)$ \\
\hline$C(14)$ & $1522(13)$ & $5572(3)$ & $2902(3)$ & $44(2)$ \\
\hline$C(15)$ & $-883(15)$ & $3228(4)$ & $3886(3)$ & $46(2)$ \\
\hline$C(16)$ & $1093(16)$ & $3676(4)$ & $4192(3)$ & $63(2)$ \\
\hline$C(17)$ & $-2953(15)$ & $3733(4)$ & $3744(3)$ & $60(2)$ \\
\hline$C(18)$ & $-1508(17)$ & $2552(4)$ & $4341(4)$ & $78(3)$ \\
\hline
\end{tabular}


Table 3. Bond lengths $[\AA]$ and angles $\left[{ }^{\circ}\right]$ for compound $\mathbf{1 8}(\boldsymbol{R}, S)$.

\begin{tabular}{|c|c|c|c|}
\hline $\mathrm{S}(1)-\mathrm{O}(1)$ & $1.484(6)$ & $\mathrm{C}(13)-\mathrm{H}(13 \mathrm{C})$ & 0.9700 \\
\hline $\mathrm{S}(1)-\mathrm{N}(1)$ & $1.653(4)$ & $\mathrm{C}(14)-\mathrm{H}(14 \mathrm{~A})$ & 0.9700 \\
\hline$S(1)-C(15)$ & $1.838(6)$ & $\mathrm{C}(14)-\mathrm{H}(14 \mathrm{~B})$ & 0.9700 \\
\hline $\mathrm{P}(1)-\mathrm{O}(2)$ & $1.444(5)$ & $\mathrm{C}(14)-\mathrm{H}(14 \mathrm{C})$ & 0.9700 \\
\hline $\mathrm{P}(1)-\mathrm{O}(4)$ & $1.569(4)$ & $\mathrm{C}(15)-\mathrm{C}(17)$ & $1.517(10)$ \\
\hline $\mathrm{P}(1)-\mathrm{O}(3)$ & $1.583(3)$ & $\mathrm{C}(15)-\mathrm{C}(16)$ & $1.517(11)$ \\
\hline $\mathrm{P}(1)-\mathrm{C}(12)$ & $1.789(6)$ & $\mathrm{C}(15)-\mathrm{C}(18)$ & $1.528(8)$ \\
\hline $\mathrm{O}(3)-\mathrm{C}(13)$ & $1.452(7)$ & $\mathrm{C}(16)-\mathrm{H}(16 \mathrm{~A})$ & 0.9700 \\
\hline $\mathrm{O}(4)-\mathrm{C}(14)$ & $1.461(8)$ & $\mathrm{C}(16)-\mathrm{H}(16 \mathrm{~B})$ & 0.9700 \\
\hline $\mathrm{N}(1)-\mathrm{C}(1)$ & $1.468(7)$ & $\mathrm{C}(16)-\mathrm{H}(16 \mathrm{C})$ & 0.9700 \\
\hline $\mathrm{N}(1)-\mathrm{H}(1 \mathrm{~N})$ & $0.89(2)$ & $\mathrm{C}(17)-\mathrm{H}(17 \mathrm{~A})$ & 0.9700 \\
\hline $\mathrm{C}(1)-\mathrm{C}(2)$ & $1.525(7)$ & $\mathrm{C}(17)-\mathrm{H}(17 \mathrm{~B})$ & 0.9700 \\
\hline $\mathrm{C}(1)-\mathrm{C}(12)$ & $1.541(6)$ & $\mathrm{C}(17)-\mathrm{H}(17 \mathrm{C})$ & 0.9700 \\
\hline $\mathrm{C}(1)-\mathrm{H}(1)$ & 0.9900 & $\mathrm{C}(18)-\mathrm{H}(18 \mathrm{~A})$ & 0.9700 \\
\hline$C(2)-C(3)$ & $1.358(8)$ & $\mathrm{C}(18)-\mathrm{H}(18 \mathrm{~B})$ & 0.9700 \\
\hline$C(2)-C(11)$ & $1.408(8)$ & $\mathrm{C}(18)-\mathrm{H}(18 \mathrm{C})$ & 0.9700 \\
\hline $\mathrm{C}(3)-\mathrm{C}(4)$ & $1.436(7)$ & & \\
\hline $\mathrm{C}(3)-\mathrm{H}(3)$ & 0.9400 & $\mathrm{O}(1)-\mathrm{S}(1)-\mathrm{N}(1)$ & $111.4(3)$ \\
\hline $\mathrm{C}(4)-\mathrm{C}(9)$ & $1.400(8)$ & $\mathrm{O}(1)-\mathrm{S}(1)-\mathrm{C}(15)$ & 104.2(4) \\
\hline $\mathrm{C}(4)-\mathrm{C}(5)$ & $1.405(8)$ & $\mathrm{N}(1)-\mathrm{S}(1)-\mathrm{C}(15)$ & $99.0(2)$ \\
\hline$C(5)-C(6)$ & $1.375(8)$ & $\mathrm{O}(2)-\mathrm{P}(1)-\mathrm{O}(4)$ & 109.8(3) \\
\hline $\mathrm{C}(5)-\mathrm{H}(5)$ & 0.9400 & $\mathrm{O}(2)-\mathrm{P}(1)-\mathrm{O}(3)$ & $114.4(3)$ \\
\hline$C(6)-C(7)$ & 1.371(10) & $\mathrm{O}(4)-\mathrm{P}(1)-\mathrm{O}(3)$ & $107.5(2)$ \\
\hline $\mathrm{C}(6)-\mathrm{H}(6)$ & 0.9400 & $\mathrm{O}(2)-\mathrm{P}(1)-\mathrm{C}(12)$ & $113.5(3)$ \\
\hline $\mathrm{C}(7)-\mathrm{C}(8)$ & $1.366(9)$ & $\mathrm{O}(4)-\mathrm{P}(1)-\mathrm{C}(12)$ & $111.0(3)$ \\
\hline $\mathrm{C}(7)-\mathrm{H}(7)$ & 0.9400 & $\mathrm{O}(3)-\mathrm{P}(1)-\mathrm{C}(12)$ & $100.2(2)$ \\
\hline $\mathrm{C}(8)-\mathrm{C}(9)$ & $1.429(7)$ & $\mathrm{C}(13)-\mathrm{O}(3)-\mathrm{P}(1)$ & $118.6(4)$ \\
\hline $\mathrm{C}(8)-\mathrm{H}(8)$ & 0.9400 & $\mathrm{C}(14)-\mathrm{O}(4)-\mathrm{P}(1)$ & 121.1(4) \\
\hline $\mathrm{C}(9)-\mathrm{C}(10)$ & $1.411(8)$ & $\mathrm{C}(1)-\mathrm{N}(1)-\mathrm{S}(1)$ & $114.7(3)$ \\
\hline$C(10)-C(11)$ & $1.366(7)$ & $\mathrm{C}(1)-\mathrm{N}(1)-\mathrm{H}(1 \mathrm{~N})$ & $122(4)$ \\
\hline $\mathrm{C}(10)-\mathrm{H}(10)$ & 0.9400 & $\mathrm{~S}(1)-\mathrm{N}(1)-\mathrm{H}(1 \mathrm{~N})$ & $102(4)$ \\
\hline $\mathrm{C}(11)-\mathrm{H}(11)$ & 0.9400 & $\mathrm{~N}(1)-\mathrm{C}(1)-\mathrm{C}(2)$ & $114.9(4)$ \\
\hline $\mathrm{C}(12)-\mathrm{H}(12 \mathrm{~A})$ & 0.9800 & $\mathrm{~N}(1)-\mathrm{C}(1)-\mathrm{C}(12)$ & 108.1(4) \\
\hline $\mathrm{C}(12)-\mathrm{H}(12 \mathrm{~B})$ & 0.9800 & $\mathrm{C}(2)-\mathrm{C}(1)-\mathrm{C}(12)$ & 109.1(4) \\
\hline $\mathrm{C}(13)-\mathrm{H}(13 \mathrm{~A})$ & 0.9700 & $\mathrm{~N}(1)-\mathrm{C}(1)-\mathrm{H}(1)$ & 108.2 \\
\hline $\mathrm{C}(13)-\mathrm{H}(13 \mathrm{~B})$ & 0.9700 & $\mathrm{C}(2)-\mathrm{C}(1)-\mathrm{H}(1)$ & 108.2 \\
\hline
\end{tabular}




\begin{tabular}{|c|c|}
\hline $\mathrm{C}(12)-\mathrm{C}(1)-\mathrm{H}(1)$ & 108.2 \\
\hline$C(3)-C(2)-C(11)$ & $120.1(5)$ \\
\hline $\mathrm{C}(3)-\mathrm{C}(2)-\mathrm{C}(1)$ & $119.7(5)$ \\
\hline $\mathrm{C}(11)-\mathrm{C}(2)-\mathrm{C}(1)$ & $120.3(5)$ \\
\hline$C(2)-C(3)-C(4)$ & $121.0(5)$ \\
\hline $\mathrm{C}(2)-\mathrm{C}(3)-\mathrm{H}(3)$ & 119.5 \\
\hline $\mathrm{C}(4)-\mathrm{C}(3)-\mathrm{H}(3)$ & 119.5 \\
\hline$C(9)-C(4)-C(5)$ & $119.7(5)$ \\
\hline$C(9)-C(4)-C(3)$ & $118.3(5)$ \\
\hline$C(5)-C(4)-C(3)$ & $121.9(6)$ \\
\hline$C(6)-C(5)-C(4)$ & $119.8(7)$ \\
\hline $\mathrm{C}(6)-\mathrm{C}(5)-\mathrm{H}(5)$ & 120.1 \\
\hline $\mathrm{C}(4)-\mathrm{C}(5)-\mathrm{H}(5)$ & 120.1 \\
\hline$C(7)-C(6)-C(5)$ & $121.0(6)$ \\
\hline $\mathrm{C}(7)-\mathrm{C}(6)-\mathrm{H}(6)$ & 119.5 \\
\hline $\mathrm{C}(5)-\mathrm{C}(6)-\mathrm{H}(6)$ & 119.5 \\
\hline$C(8)-C(7)-C(6)$ & $121.0(5)$ \\
\hline $\mathrm{C}(8)-\mathrm{C}(7)-\mathrm{H}(7)$ & 119.5 \\
\hline $\mathrm{C}(6)-\mathrm{C}(7)-\mathrm{H}(7)$ & 119.5 \\
\hline$C(7)-C(8)-C(9)$ & $119.7(7)$ \\
\hline $\mathrm{C}(7)-\mathrm{C}(8)-\mathrm{H}(8)$ & 120.1 \\
\hline $\mathrm{C}(9)-\mathrm{C}(8)-\mathrm{H}(8)$ & 120.1 \\
\hline $\mathrm{C}(4)-\mathrm{C}(9)-\mathrm{C}(10)$ & $119.4(5)$ \\
\hline $\mathrm{C}(4)-\mathrm{C}(9)-\mathrm{C}(8)$ & $118.7(5)$ \\
\hline$C(10)-C(9)-C(8)$ & $121.9(6)$ \\
\hline $\mathrm{C}(11)-\mathrm{C}(10)-\mathrm{C}(9)$ & $121.0(6)$ \\
\hline $\mathrm{C}(11)-\mathrm{C}(10)-\mathrm{H}(10)$ & 119.5 \\
\hline $\mathrm{C}(9)-\mathrm{C}(10)-\mathrm{H}(10)$ & 119.5 \\
\hline$C(10)-C(11)-C(2)$ & $120.2(5)$ \\
\hline $\mathrm{C}(10)-\mathrm{C}(11)-\mathrm{H}(11)$ & 119.9 \\
\hline $\mathrm{C}(2)-\mathrm{C}(11)-\mathrm{H}(11)$ & 119.9 \\
\hline $\mathrm{C}(1)-\mathrm{C}(12)-\mathrm{P}(1)$ & $114.9(4)$ \\
\hline $\mathrm{C}(1)-\mathrm{C}(12)-\mathrm{H}(12 \mathrm{~A})$ & 108.5 \\
\hline $\mathrm{P}(1)-\mathrm{C}(12)-\mathrm{H}(12 \mathrm{~A})$ & 108.5 \\
\hline $\mathrm{C}(1)-\mathrm{C}(12)-\mathrm{H}(12 \mathrm{~B})$ & 108.5 \\
\hline $\mathrm{P}(1)-\mathrm{C}(12)-\mathrm{H}(12 \mathrm{~B})$ & 108.5 \\
\hline $\mathrm{H}(12 \mathrm{~A})-\mathrm{C}(12)-\mathrm{H}(12 \mathrm{~B})$ & 107.5 \\
\hline $\mathrm{O}(3)-\mathrm{C}(13)-\mathrm{H}(13 \mathrm{~A})$ & 109.5 \\
\hline
\end{tabular}

$\mathrm{O}(3)-\mathrm{C}(13)-\mathrm{H}(13 \mathrm{~B}) \quad 109.5$

$\mathrm{H}(13 \mathrm{~A})-\mathrm{C}(13)-\mathrm{H}(13 \mathrm{~B}) \quad 109.5$

$\mathrm{O}(3)-\mathrm{C}(13)-\mathrm{H}(13 \mathrm{C}) \quad 109.5$

$\mathrm{H}(13 \mathrm{~A})-\mathrm{C}(13)-\mathrm{H}(13 \mathrm{C}) \quad 109.5$

H(13B)-C(13)-H(13C) $\quad 109.5$

$\mathrm{O}(4)-\mathrm{C}(14)-\mathrm{H}(14 \mathrm{~A}) \quad 109.5$

$\mathrm{O}(4)-\mathrm{C}(14)-\mathrm{H}(14 \mathrm{~B}) \quad 109.5$

$\mathrm{H}(14 \mathrm{~A})-\mathrm{C}(14)-\mathrm{H}(14 \mathrm{~B}) \quad 109.5$

$\mathrm{O}(4)-\mathrm{C}(14)-\mathrm{H}(14 \mathrm{C}) \quad 109.5$

H(14A)-C(14)-H(14C) 109.5

H(14B)-C(14)-H(14C) 109.5

C(17)-C(15)-C(16) 112.5(6)

C(17)-C(15)-C(18) 111.4(7)

C(16)-C(15)-C(18) 109.5(6)

C(17)-C(15)-S(1) 110.5(4)

C(16)-C(15)-S(1) 108.2(5)

C(18)-C(15)-S(1) 104.4(5)

C(15)-C(16)-H(16A) $\quad 109.5$

C(15)-C(16)-H(16B) 109.5

H(16A)-C(16)-H(16B) 109.5

$\mathrm{C}(15)-\mathrm{C}(16)-\mathrm{H}(16 \mathrm{C}) \quad 109.5$

H(16A)-C(16)-H(16C) 109.5

H(16B)-C(16)-H(16C) $\quad 109.5$

$\mathrm{C}(15)-\mathrm{C}(17)-\mathrm{H}(17 \mathrm{~A}) \quad 109.5$

$\mathrm{C}(15)-\mathrm{C}(17)-\mathrm{H}(17 \mathrm{~B}) \quad 109.5$

H(17A)-C(17)-H(17B) $\quad 109.5$

$\mathrm{C}(15)-\mathrm{C}(17)-\mathrm{H}(17 \mathrm{C}) \quad 109.5$

H(17A)-C(17)-H(17C) $\quad 109.5$

H(17B)-C(17)-H(17C) $\quad 109.5$

$\mathrm{C}(15)-\mathrm{C}(18)-\mathrm{H}(18 \mathrm{~A}) \quad 109.5$

$\mathrm{C}(15)-\mathrm{C}(18)-\mathrm{H}(18 \mathrm{~B}) \quad 109.5$

H(18A)-C(18)-H(18B) $\quad 109.5$

$\mathrm{C}(15)-\mathrm{C}(18)-\mathrm{H}(18 \mathrm{C}) \quad 109.5$

$\mathrm{H}(18 \mathrm{~A})-\mathrm{C}(18)-\mathrm{H}(18 \mathrm{C}) \quad 109.5$

H(18B)-C(18)-H(18C) $\quad 109.5$ 
Symmetry transformations used to generate equivalent atoms:

Table 4. Anisotropic displacement parameters $\left(\AA^{2} \times 10^{3}\right)$ for compound $\mathbf{1 8}(\boldsymbol{R}, \boldsymbol{S})$. The anisotropic displacement factor exponent takes the form: $-2 \pi^{2}\left[h^{2} a^{* 2} U^{11}+\ldots+2 h k a^{*} b^{*} U^{12}\right]$

\begin{tabular}{|c|c|c|c|c|c|c|}
\hline & $\mathrm{U}^{11}$ & $\mathrm{U}^{22}$ & $\mathrm{U}^{33}$ & $\mathrm{U}^{23}$ & $\mathrm{U}^{13}$ & $\mathrm{U}^{12}$ \\
\hline$S(1)$ & $60(2)$ & $30(1)$ & $48(1)$ & 13(1) & $25(1)$ & 11(1) \\
\hline $\mathrm{P}(1)$ & $13(1)$ & $23(1)$ & $36(1)$ & $-1(1)$ & $0(1)$ & $0(1)$ \\
\hline $\mathrm{O}(1)$ & $117(6)$ & $50(3)$ & $68(3)$ & $-13(2)$ & $35(4)$ & $-44(3)$ \\
\hline $\mathrm{O}(2)$ & $30(3)$ & $34(2)$ & $58(3)$ & $-2(2)$ & $-3(3)$ & $4(2)$ \\
\hline $\mathrm{O}(3)$ & $29(3)$ & $28(2)$ & $52(2)$ & $8(2)$ & $-6(2)$ & $-4(2)$ \\
\hline $\mathrm{O}(4)$ & $25(3)$ & $39(2)$ & $35(2)$ & $-9(2)$ & $-6(2)$ & $5(2)$ \\
\hline $\mathrm{N}(1)$ & $20(3)$ & $22(2)$ & $35(2)$ & $-3(2)$ & $6(3)$ & $4(2)$ \\
\hline $\mathrm{C}(1)$ & 11(4) & $25(2)$ & $24(3)$ & $-3(2)$ & 2(3) & $0(2)$ \\
\hline$C(2)$ & $22(4)$ & $23(2)$ & $26(3)$ & $2(2)$ & 2(3) & $-7(2)$ \\
\hline $\mathrm{C}(3)$ & $20(4)$ & $26(2)$ & $23(3)$ & $4(2)$ & $-5(3)$ & $-1(2)$ \\
\hline $\mathrm{C}(4)$ & 21(4) & $22(2)$ & $26(3)$ & $0(2)$ & $-1(3)$ & $-2(2)$ \\
\hline $\mathrm{C}(5)$ & $37(5)$ & $31(3)$ & $45(4)$ & $0(3)$ & $-4(3)$ & $5(3)$ \\
\hline$C(6)$ & $72(7)$ & $30(3)$ & $39(3)$ & $-4(3)$ & $6(4)$ & $10(3)$ \\
\hline$C(7)$ & $71(6)$ & $31(3)$ & $29(3)$ & $-8(2)$ & $-4(4)$ & $0(3)$ \\
\hline $\mathrm{C}(8)$ & $35(5)$ & $37(3)$ & $29(3)$ & $-1(2)$ & $-5(3)$ & $-9(3)$ \\
\hline $\mathrm{C}(9)$ & $24(5)$ & $27(2)$ & $25(2)$ & $3(2)$ & 4(3) & $-5(2)$ \\
\hline $\mathrm{C}(10)$ & $22(5)$ & $38(3)$ & $35(3)$ & $6(2)$ & $-6(3)$ & $-4(3)$ \\
\hline $\mathrm{C}(11)$ & $16(4)$ & $26(2)$ & $38(3)$ & $0(2)$ & 1(3) & $5(2)$ \\
\hline $\mathrm{C}(12)$ & $26(4)$ & $27(3)$ & $31(3)$ & $-2(2)$ & $-6(3)$ & $-5(3)$ \\
\hline $\mathrm{C}(13)$ & $50(6)$ & $30(3)$ & $82(5)$ & $10(3)$ & $5(4)$ & $-17(3)$ \\
\hline $\mathrm{C}(14)$ & $34(5)$ & $41(3)$ & $58(4)$ & $-22(3)$ & $0(4)$ & $4(3)$ \\
\hline$C(15)$ & $38(6)$ & $67(4)$ & $31(3)$ & $7(3)$ & $6(4)$ & $0(4)$ \\
\hline$C(16)$ & $52(7)$ & $97(5)$ & $41(4)$ & $7(4)$ & $5(4)$ & $-11(5)$ \\
\hline $\mathrm{C}(17)$ & $41(7)$ & $93(5)$ & $44(4)$ & $-14(4)$ & $15(4)$ & $10(4)$ \\
\hline $\mathrm{C}(18)$ & $76(8)$ & $109(7)$ & $48(4)$ & $27(4)$ & $8(5)$ & $-27(5)$ \\
\hline
\end{tabular}


Table 5. Hydrogen coordinates $\left(\mathrm{x} 10^{4}\right)$ and isotropic displacement parameters $\left(\AA^{2} \times 10^{3}\right)$ for compound $\mathbf{1 8}(R, S)$.

\begin{tabular}{|c|c|c|c|c|}
\hline & $\mathrm{x}$ & $\mathrm{y}$ & $\mathrm{z}$ & $\mathrm{U}(\mathrm{eq})$ \\
\hline $\mathrm{H}(1 \mathrm{~N})$ & $-840(50)$ & $3740(30)$ & $2610(30)$ & 31 \\
\hline $\mathrm{H}(1)$ & 3663 & 3291 & 2268 & 24 \\
\hline $\mathrm{H}(3)$ & 4143 & 2190 & 1662 & 28 \\
\hline $\mathrm{H}(5)$ & 4935 & 1002 & 983 & 45 \\
\hline $\mathrm{H}(6)$ & 3825 & 154 & 154 & 56 \\
\hline $\mathrm{H}(7)$ & 431 & 324 & -411 & 52 \\
\hline $\mathrm{H}(8)$ & -2031 & 1310 & -133 & 40 \\
\hline $\mathrm{H}(10)$ & -2821 & 2493 & 552 & 38 \\
\hline $\mathrm{H}(11)$ & -1705 & 3366 & 1353 & 32 \\
\hline $\mathrm{H}(12 \mathrm{~A})$ & 964 & 4505 & 1739 & 34 \\
\hline $\mathrm{H}(12 \mathrm{~B})$ & 2905 & 4151 & 1279 & 34 \\
\hline $\mathrm{H}(13 \mathrm{~A})$ & 7325 & 6017 & 1981 & 81 \\
\hline $\mathrm{H}(13 \mathrm{~B})$ & 5335 & 6635 & 1917 & 81 \\
\hline $\mathrm{H}(13 \mathrm{C})$ & 6633 & 6343 & 1269 & 81 \\
\hline $\mathrm{H}(14 \mathrm{~A})$ & 1592 & 6043 & 2641 & 67 \\
\hline $\mathrm{H}(14 \mathrm{~B})$ & 1285 & 5699 & 3369 & 67 \\
\hline $\mathrm{H}(14 \mathrm{C})$ & 260 & 5256 & 2744 & 67 \\
\hline $\mathrm{H}(16 \mathrm{~A})$ & 1395 & 4133 & 3926 & 95 \\
\hline $\mathrm{H}(16 \mathrm{~B})$ & 2453 & 3354 & 4203 & 95 \\
\hline $\mathrm{H}(16 \mathrm{C})$ & 692 & 3829 & 4644 & 95 \\
\hline $\mathrm{H}(17 \mathrm{~A})$ & -3636 & 3897 & 4163 & 89 \\
\hline $\mathrm{H}(17 \mathrm{~B})$ & -4072 & 3443 & 3488 & 89 \\
\hline $\mathrm{H}(17 \mathrm{C})$ & -2476 & 4183 & 3491 & 89 \\
\hline $\mathrm{H}(18 \mathrm{~A})$ & -194 & 2212 & 4386 & 117 \\
\hline $\mathrm{H}(18 \mathrm{~B})$ & -2781 & 2269 & 4147 & 117 \\
\hline $\mathrm{H}(18 \mathrm{C})$ & -1947 & 2745 & 4778 & 117 \\
\hline
\end{tabular}


Table 6. Torsion angles $\left[{ }^{\circ}\right]$ for compound $18(R, S)$.

\begin{tabular}{|c|c|}
\hline $\mathrm{O}(2)-\mathrm{P}(1)-\mathrm{O}(3)-\mathrm{C}(13)$ & $-50.1(5)$ \\
\hline $\mathrm{O}(4)-\mathrm{P}(1)-\mathrm{O}(3)-\mathrm{C}(13)$ & $72.2(5)$ \\
\hline $\mathrm{C}(12)-\mathrm{P}(1)-\mathrm{O}(3)-\mathrm{C}(13)$ & $-171.8(5)$ \\
\hline $\mathrm{O}(2)-\mathrm{P}(1)-\mathrm{O}(4)-\mathrm{C}(14)$ & $175.0(4)$ \\
\hline $\mathrm{O}(3)-\mathrm{P}(1)-\mathrm{O}(4)-\mathrm{C}(14)$ & $50.0(5)$ \\
\hline $\mathrm{C}(12)-\mathrm{P}(1)-\mathrm{O}(4)-\mathrm{C}(14)$ & $-58.7(4)$ \\
\hline $\mathrm{O}(1)-\mathrm{S}(1)-\mathrm{N}(1)-\mathrm{C}(1)$ & $-91.1(5)$ \\
\hline $\mathrm{C}(15)-\mathrm{S}(1)-\mathrm{N}(1)-\mathrm{C}(1)$ & $159.8(4)$ \\
\hline $\mathrm{S}(1)-\mathrm{N}(1)-\mathrm{C}(1)-\mathrm{C}(2)$ & $60.2(6)$ \\
\hline $\mathrm{S}(1)-\mathrm{N}(1)-\mathrm{C}(1)-\mathrm{C}(12)$ & $-177.6(4)$ \\
\hline $\mathrm{N}(1)-\mathrm{C}(1)-\mathrm{C}(2)-\mathrm{C}(3)$ & $-122.3(5)$ \\
\hline $\mathrm{C}(12)-\mathrm{C}(1)-\mathrm{C}(2)-\mathrm{C}(3)$ & 116.1(5) \\
\hline $\mathrm{N}(1)-\mathrm{C}(1)-\mathrm{C}(2)-\mathrm{C}(11)$ & $59.0(6)$ \\
\hline $\mathrm{C}(12)-\mathrm{C}(1)-\mathrm{C}(2)-\mathrm{C}(11)$ & $-62.7(6)$ \\
\hline $\mathrm{C}(11)-\mathrm{C}(2)-\mathrm{C}(3)-\mathrm{C}(4)$ & $1.6(7)$ \\
\hline $\mathrm{C}(1)-\mathrm{C}(2)-\mathrm{C}(3)-\mathrm{C}(4)$ & $-177.2(4)$ \\
\hline $\mathrm{C}(2)-\mathrm{C}(3)-\mathrm{C}(4)-\mathrm{C}(9)$ & $-0.6(7)$ \\
\hline $\mathrm{C}(2)-\mathrm{C}(3)-\mathrm{C}(4)-\mathrm{C}(5)$ & $178.8(5)$ \\
\hline $\mathrm{C}(9)-\mathrm{C}(4)-\mathrm{C}(5)-\mathrm{C}(6)$ & $0.5(8)$ \\
\hline$C(3)-C(4)-C(5)-C(6)$ & $-178.9(5)$ \\
\hline $\mathrm{C}(4)-\mathrm{C}(5)-\mathrm{C}(6)-\mathrm{C}(7)$ & $0.0(10)$ \\
\hline$C(5)-C(6)-C(7)-C(8)$ & $-1.4(10)$ \\
\hline $\mathrm{C}(6)-\mathrm{C}(7)-\mathrm{C}(8)-\mathrm{C}(9)$ & $2.2(9)$ \\
\hline $\mathrm{C}(5)-\mathrm{C}(4)-\mathrm{C}(9)-\mathrm{C}(10)$ & $179.6(5)$ \\
\hline $\mathrm{C}(3)-\mathrm{C}(4)-\mathrm{C}(9)-\mathrm{C}(10)$ & $-0.9(7)$ \\
\hline $\mathrm{C}(5)-\mathrm{C}(4)-\mathrm{C}(9)-\mathrm{C}(8)$ & $0.3(7)$ \\
\hline$C(3)-C(4)-C(9)-C(8)$ & $179.7(5)$ \\
\hline $\mathrm{C}(7)-\mathrm{C}(8)-\mathrm{C}(9)-\mathrm{C}(4)$ & $-1.6(8)$ \\
\hline $\mathrm{C}(7)-\mathrm{C}(8)-\mathrm{C}(9)-\mathrm{C}(10)$ & 179.1(6) \\
\hline $\mathrm{C}(4)-\mathrm{C}(9)-\mathrm{C}(10)-\mathrm{C}(11)$ & $1.5(8)$ \\
\hline $\mathrm{C}(8)-\mathrm{C}(9)-\mathrm{C}(10)-\mathrm{C}(11)$ & $-179.2(5)$ \\
\hline $\mathrm{C}(9)-\mathrm{C}(10)-\mathrm{C}(11)-\mathrm{C}(2)$ & $-0.5(8)$ \\
\hline$C(3)-C(2)-C(11)-C(10)$ & $-1.0(8)$ \\
\hline
\end{tabular}


$\mathrm{C}(1)-\mathrm{C}(2)-\mathrm{C}(11)-\mathrm{C}(10)$

$\mathrm{N}(1)-\mathrm{C}(1)-\mathrm{C}(12)-\mathrm{P}(1)$

$\mathrm{C}(2)-\mathrm{C}(1)-\mathrm{C}(12)-\mathrm{P}(1)$

$\mathrm{O}(2)-\mathrm{P}(1)-\mathrm{C}(12)-\mathrm{C}(1)$

$\mathrm{O}(4)-\mathrm{P}(1)-\mathrm{C}(12)-\mathrm{C}(1)$

$\mathrm{O}(3)-\mathrm{P}(1)-\mathrm{C}(12)-\mathrm{C}(1)$

$\mathrm{O}(1)-\mathrm{S}(1)-\mathrm{C}(15)-\mathrm{C}(17)$

$\mathrm{N}(1)-\mathrm{S}(1)-\mathrm{C}(15)-\mathrm{C}(17)$

$\mathrm{O}(1)-\mathrm{S}(1)-\mathrm{C}(15)-\mathrm{C}(16)$

$\mathrm{N}(1)-\mathrm{S}(1)-\mathrm{C}(15)-\mathrm{C}(16)$

$\mathrm{O}(1)-\mathrm{S}(1)-\mathrm{C}(15)-\mathrm{C}(18)$

$\mathrm{N}(1)-\mathrm{S}(1)-\mathrm{C}(15)-\mathrm{C}(18)$
177.7(5)

$82.2(5)$

$-152.1(4)$

56.1(5)

$-68.2(5)$

178.5(4)

$-58.2(5)$

$56.6(6)$

178.2(5)

$-67.0(5)$

61.6(6)

176.5(6)

Symmetry transformations used to generate equivalent atoms:

Table 7. Hydrogen bonds for compound $18(\boldsymbol{R}, S)\left[\AA\right.$ and $\left.{ }^{\circ}\right]$.

\begin{tabular}{lllll}
\hline D-H...A & $\mathrm{d}(\mathrm{D}-\mathrm{H})$ & $\mathrm{d}(\mathrm{H} \ldots \mathrm{A})$ & $\mathrm{d}(\mathrm{D} \ldots \mathrm{A})$ & $<(\mathrm{DHA})$ \\
\hline $\mathrm{N}(1)-\mathrm{H}(1 \mathrm{~N}) \ldots \mathrm{O}(2) \# 1$ & $0.89(2)$ & $2.13(3)$ & $2.943(7)$ & $151(5)$ \\
& & & & \\
\hline
\end{tabular}

Symmetry transformations used to generate equivalent atoms:

\#1 x-1,y,z 